\title{
Computations of Turbulent Premixed Flames Using Conditional Moment Closure
}

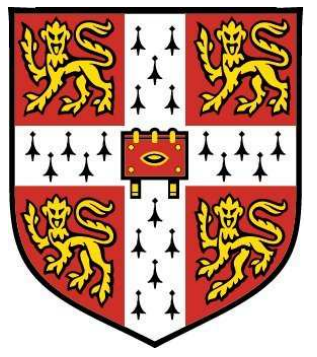

\author{
Shokri Amzin \\ Department of Engineering \\ University of Cambridge
}

A thesis submitted for the degree of

Doctor of Philosophy

February 2012 
I would like to dedicate this Doctoral

dissertation to my parents and my family for their moral support and continued love for my entire life. Most importantly, to my beautiful wife Catharina and my gorgeous son Shaheer for their love and patience. 


\section{Declaration}

I hereby declare that the work presented in this dissertation is the result of my own work includes nothing which is the outcome of work done in collaboration except where specifically indicated in the text by a reference. No part of this work has been submitted for any other degree or diploma.

This dissertation contains approximately 38000 words and 56 figures.

Hopkinson Laboratory, Cambridge

Shokri Amzin

28th February 2012 


\section{Acknowledgements}

It has been an honor to work with Dr. N. Swaminathan during my $\mathrm{PhD}$. I am very grateful for his guidance, advice and supervision from the initial to the final level which, enabled me to develop an understanding of turbulent combustion modelling.

I am also heartily thankful to all those who assisted me in the preparation and completion of this study. I mention here, Dr. Jim Rogerson and Dr. Hemanth Kolla for their continuous support.

I also wish to extend my sincere gratitude to Professors Nondas Mastorakos, Stewart Cant, Nick Collings and Simone Hochgreb for their assistance and guidance through the combustion courses.

I owe my deepest gratitude to Mr. Peter Benie for his IT support and Mrs. Kate Graham for her assistance. Sincere thanks to my friends and colleagues members of Heat gallery and Hopkinson Labs.

Finally, the generous financial support of Cambridge Commonwealth Trust, Siemens and EPSRC is acknowledge gratefully. This dissertation would not have been possible without their supports. 


\section{Publications}

The results of this thesis have been partially presented and published in:

1. S. Amzin, N. Swaminathan, J. W. Rogerson, J. H. Kent. Conditional moment closure for turbulent premixed flames. presented in 23rd ICDERS, California, Irvine, 2011.

2. S. Amzin, N. Swaminathan, J. W. Rogerson, J. H. Kent. Conditional moment closure for turbulent premixed flames. Combustion Science and Technology, 2011,(accepted).

3. S. Amzin, N. Swaminathan. Conditional moment closure for turbulent lean premixed flames. Combustion Science and Technology, 2011,(in preparation). 


\begin{abstract}
Lean premixed combustion is at present one of the most promising methods to reduce emissions and to maintain high efficiency in combustion systems. As the emission legislation becomes more stringent, modelling of turbulent premixed combustion has become an important tool for designing efficient and environmentally friendlier combustion systems. However, in order to predict these emissions reliable predictive models are required. One of the methods used for predicting pollutants is the conditional moment closure (CMC), which is suitable to predict pollutants with slow time scales. Despite the fact that CMC has been successfully applied to various non-premixed combustion systems, its application to premixed flames is not fully tested and validated. The main difficulty is associated with the modelling of the conditional scalar dissipation rate (CSDR) of the conditioning scalar, the progress variable. In premixed $\mathrm{CMC}$, this term is an important quantity and represents the rate of mixing at small scales of relevance for combustion. The numerical accuracy of the CMC method depends on the accuracy of the CSDR model. In this study, two different models for CSDR, an algebraic model and an inverse problem model, are validated using two different DNS data sets. The algebraic model along with standard $k-\varepsilon$ turbulence modelling is used
\end{abstract}


in the computations of stoichiometric and very lean pilot stabilized Bunsen flames using the RANS-CMC method. A first order closure is used for the conditional mean reaction rate. The computed nonreacting and reacting scalars are in reasonable agreement with the experiments and are consistent with earlier computations using flamlets and transported PDF methods for the stoichiometric flames, and transported PDF methods for the very lean flames. Sensitivity to chemical kinetics mechanism is also assessed. 


\section{Contents}

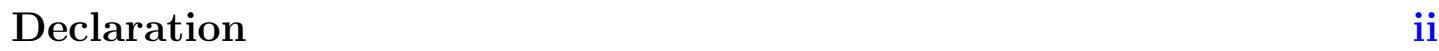

Contents vii

List of Figures $\quad x$

Nomenclature $\quad$ xvi

1 Introduction 1

1.1 Objectives ........................ 6

2 Background on Turbulent Premixed Combustion 8

2.1 Governing Equations . . . . . . . . . . . . . . . . . 10

2.1.1 Fluid Flow Equations . . . . . . . . . . . . . . . . 10

2.1.2 Reaction Rates . . . . . . . . . . . . . . . . . . 15

2.2 Three Simulation Paradigms . . . . . . . . . . . . . . 16

2.2 .1 DNS . . . . . . . . . . . . . . 16

$2.2 .2 \quad \mathrm{LES} \ldots \ldots \ldots \ldots \ldots \ldots$

2.2 .3 RANS ........................ . . 18

2.3 RANS Methodology . . . . . . . . . . . . . 18

2.4 Turbulence Models . . . . . . . . . . . . . . . . . . . . 22

2.4.1 Turbulent Reynolds Stress Model . . . . . . . . . . . . . . 22

2.4.2 Turbulent Scalar Flux Model . . . . . . . . . . . . . . . . 24

2.5 Premixed Combustion Sub-Models . . . . . . . . . . . . . 25

2.5.1 Eddy Break-Up Model . . . . . . . . . . . . . . . . . 29

2.5.2 Bray-Moss-Libby Model . . . . . . . . . . . . . . . . 30 
2.5.3 Flame Surface Density Model . . . . . . . . . . . . . . 33

2.5.4 The Probability Density Function . . . . . . . . . . 34

2.5.5 G-Equation Model . . . . . . . . . . . . . . 37

2.6 Summary . . . . . . . . . . . . . . . . . . . 37

3 Conditional Moment Closure $\quad 39$

3.1 CMC Methodology . . . . . . . . . . . . . . . . . 39

3.2 Premixed CMC Method . . . . . . . . . . . . . . . . . . 43

3.3 Premixed CMC Sub-Models . . . . . . . . . . . . . . . . 45

4 Scalar Dissipation Rate $\quad 49$

4.1 Mean Scalar Dissipation Rate . . . . . . . . . . . . . . . 50

4.2 Conditional Mean Scalar Dissipation Rate . . . . . . . . . . . 51

4.2.1 Algebraic Model . . . . . . . . . . . . . . . 51

4.2 .2 Inverse Model . . . . . . . . . . . . . . . . . . . . 52

4.3 Model Validation . . . . . . . . . . . . . . . . . . . . 54

4.3 .1 DNS Database ................. 54

4.3.2 Results and Discussion . . . . . . . . . . . . . 58

$\begin{array}{lll}5 & \text { Mathematical Model } & 61\end{array}$

5.1 Discretisation of the CMC Equation . . . . . . . . . . . . 61

5.2 Implementation for Premixed Flames . . . . . . . . . . . . . 66

5.3 Computer Model and Sequence . . . . . . . . . . . . . . . 68

6 Stoichiometric Premixed Flame Calculation $\quad 70$

6.1 Computational Details . . . . . . . . . . . . . . . 70

6.2 Results and Discussion . . . . . . . . . . . . . . . . . 75

6.2.1 Non Reacting Flow . . . . . . . . . . . . . . 75

6.2.2 Conditional Scalar Dissipation Rate . . . . . . . . . . 79

6.2.3 Terms in CMC Transport Equation . . . . . . . . . . . . . 82

6.2.4 Comparison to Experimental Results . . . . . . . . . . 85

7 Lean Premixed Flame Calculation $\quad 102$

7.1 Flames Details . . . . . . . . . . . . . . . . . . . 102

7.2 Computational Details . . . . . . . . . . . . 105 
7.3 Results and Discussion . . . . . . . . . . . . . . . . . 108

7.3.1 Conditional Mean Mass Fractions . . . . . . . . . . . 110

7.3.2 Comparison to Experimental Results . . . . . . . . . . 111

8 Summary and Conclusions 135

8.1 Conclusion From This Work . . . . . . . . . . . . . 135

8.2 Future Work . . . . . . . . . . . . . . . . . . . . . . . . 139

$\begin{array}{ll}\text { Appendix A } & 141\end{array}$

$\begin{array}{ll}\text { Bibliography } & 145\end{array}$ 


\section{List of Figures}

2.1 Structure of a laminar diffusion flames. . . . . . . . . . . . . . . . 10

2.2 Structure of a laminar premixed flames. . . . . . . . . . . . . . . . 10

2.3 The premixed combustion regime diagram. . . . . . . . . . . . . 29

3.1 Scatter plots of instantaneous and conditional averages for the temperature and $\mathrm{OH}$ mass fraction at $x / D=20$ and $r / D=1.7$ (०) ; $1.4(\square) ; 1.1(\triangle)[1]$. Solid line represents fully burning strained laminar flame result. . . . . . . . . . . . . . . . . . . . . 42

4.1 The variation of the Favre averaged PDF of the progress variable, $\tilde{p}(\zeta)$, at $\theta=0.5$ inside the flame brush of flames R2a and R2c. . . 56

4.2 The variation of the Favre averaged PDF of the progress variable, $\tilde{p}(\zeta)$, at $\theta=0.6$ inside the flame brush of flames VA and VB. . . . 57

4.3 The premixed combustion regime diagram showing the selected DNS flames. . . . . . . . . . . . . . . . . . . . 58

4.4 The typical variation of the conditional scalar dissipation rate, $\langle N \mid \zeta\rangle^{+}$, calculations obtained by, Eq. (4.7) and Eq. (4.11) with $\zeta$ in flames R2a and R2c. The values are non-dimensionalised using $S_{L}^{o}$ and $\delta_{L}^{o} \ldots \ldots \ldots \ldots \ldots \ldots \ldots$

4.5 The typical variation of the conditional scalar dissipation rate, $\langle N \mid \zeta\rangle^{+}$, obtained by, Eq. (4.7) and Eq. (4.11) with $\zeta$ in flames VA and $\mathrm{VB}$ at two different axial locations in the flame; $x_{1}=16.7 \delta_{L}^{\circ}$ and $x_{2}=27.9 \delta_{L}^{\circ}$. These values are non-dimensionalised using $S_{L}^{o}$

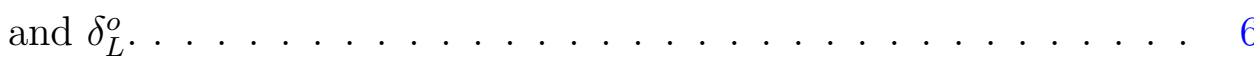


5.1 Control volume in a finite volume method. . . . . . . . . . . . . . 62

5.2 Interaction of CMC and RANS equation in the simulation. . . . . 68

6.1 Schematic diagram of the burner setup [2] and the computational domain along with BCs. . . . . . . . . . . . . 72

6.2 The premixed combustion regime diagram showing flames F1, F2 and F3. . . . . . . . . . . . . . . . . . 73

6.3 The normalised mean velocity (lines) from non-reacting flow are compared to the the experimental measurements (symbols) for flames F1, F2 and F3. . . . . . . . . . . . . . . . 77

6.4 The normalised mean turbulent kinetic energy (lines) from nonreacting flow are compared to the experimental measurements (symbols) for flames F1, F2 and F3. . . . . . . . . . . . . 78

6.5 Computed radial variation of $\widetilde{Z}$ in cold flow simulation at five axial locations for the conditions of flame F1. . . . . . . . . . 79

6.6 The typical variation of the conditional scalar dissipation rate, $\langle N \mid \zeta\rangle^{+}$, obtained in CMC calculations by, Eq. (4.7), with $\zeta$ in flame F1 at different locations inside the flame brush at $x / D=4.5$. The values are non-dimensionalised using $S_{L}^{o}$ and $\delta_{L}^{o} \ldots \ldots \ldots . . . . .80$

6.7 The typical variation of the conditional scalar dissipation rate, $\langle N \mid \zeta\rangle^{+}$, obtained in CMC calculations by, Eq. (4.7), with $\zeta$ in flame F1 at different locations inside the flame brush at $x / D=8.5$. The values are non-dimensionalised using $S_{L}^{o}$ and $\delta_{L}^{o} \ldots \ldots \ldots$. . . . . . 81

6.8 Typical variation of different terms of the CMC transport equation, Eq. (6.2), with $\zeta$ in flame $\mathrm{F} 1$ at $\tilde{\theta}=0.55$. The results are shown for few representative scalars. . . . . . . . . . . .

6.9 The variation of the conditional mean mass fraction with $\zeta$ for major and minor species in flame $\mathrm{F} 1$ at $\tilde{\theta}=0.55$ and $x / d=4.5 . \quad .84$

6.10 The variation of the conditional mean mass fraction with $\zeta$ for major and minor species in flame $\mathrm{F} 1$ at $\tilde{\theta}=0.55$ and $x / d=8.5 . \quad .84$

6.11 The variation of the conditional mean mass fraction with $\zeta$ for major and minor species in flame $\mathrm{F} 1$ at $\tilde{\theta}=0.55$ and $x / d=10.5$. 
6.12 The variation of the Favre averaged PDF of the progress variable, $\tilde{p}(\zeta)$, at three locations in the flame brush of flame F1. . . . . . 86

6.13 Contours of the mean progress variable and its variance for flame F1 87

6.14 Contours of the mean mixture fraction variable and temperature for flame $\mathrm{F} 1 \ldots \ldots$. . . . . . . . . . . . . . . . . . . 88

6.15 The normalised mean velocity and turbulent kinetic energy from the CMC calculations (lines) at five axial locations are compared to experimental results (symbols) for flame F1. . . . . . . . . . . . 90

6.16 The normalised mean temperature and $\mathrm{CH}_{4}$ mass fraction $\times 100$, from the CMC calculations (lines) at five axial locations are compared to experimental results (symbols) for flame F1. . . . . . . . 92

6.17 The mean mass fractions of $\mathrm{H}_{2} \mathrm{O}, \mathrm{CO}_{2}$ and $\mathrm{O}_{2}$ from the $\mathrm{CMC}$ calculations (lines) at five axial locations are compared to experimental results (symbols) for flame F1. . . . . . . . . . . .

6.18 The mean mass fractions of $\mathrm{CO} \times 10, \mathrm{OH} \times 75$ and $\mathrm{H}_{2} \times 100$ from the CMC calculations (lines) at five axial locations are compared to experimental results (symbols) for flame F1. . . . . . . . . . . 94

6.19 The normalised mean velocity from the CMC calculations (lines) at five axial locations are compared to experimental results (symbols) for flames $\mathrm{F} 2$ and $\mathrm{F} 3 . \quad \ldots \ldots \ldots$. . . . . . . . . . . . 96

6.20 The normalised mean turbulent kinetic energy from the CMC calculations (lines) at five axial locations are compared to experimental results (symbols) for flames F2 and F3. . . . . . . . . . . 97

6.21 The normalised mean mass fraction of $\mathrm{CH}_{4} \times 100$ from the $\mathrm{CMC}$ calculations (lines) at five axial locations are compared to experimental results (symbols) for flames F2 and F3. . . . . . . . . . 98

6.22 The normalised mean temperature from the CMC calculations (lines) at five axial locations are compared to experimental results (symbols) for flames F2 and F3. . . . . . . . . . . . . . . . 99

6.23 The mean mass fractions of $\mathrm{H}_{2} \mathrm{O}(\triangle,-), \mathrm{CO}_{2}(\circ, \cdots)$ and $\mathrm{O}_{2}(\bullet,-$. -) from the CMC calculations (lines) at five axial locations are compared to experimental results (symbols) for flames F2 and F3. 100 
6.24 The mean mass fractions of $\mathrm{CO} \times 10(\circ, \cdots), \mathrm{OH} \times 75(\triangle,-)$ and $\mathrm{H}_{2} \times 100(\bullet,-\cdot-)$ from the CMC calculations (solid lines) at five axial locations are compared to experimental results (symbols) for flames F2 and F3. . . . . . . . . . . . . . . . . . . . . . . . 101

7.1 Schematic diagram of the premixed Bunsen burner from [3; 4]. . . 103

7.2 The premixed combustion regime diagram showing the stoichiometric and lean flames. . . . . . . . . . . . . . . . . 105

7.3 Schematic diagram of the burner setup and the computational domain along with BCs. . . . . . . . . . . . . . . . . 107

7.4 The calculated (lines) normalised mean axial velocity and rms velocity from non-reacting and reacting flows are compared to experimental data (symbols) for flame PM1-200. . . . . . . . . . . . . . 109

7.5 The calculated (lines) normalised mean axial velocity and rms velocity from non-reacting and reacting flows are compared to experimental data (symbols) for flame PM1-50. . . . . . . . . . . . . 110

7.6 The variation of the conditional mean mass fraction with $\zeta$ for major and minor species for flame PM1-200 at $\tilde{\theta}=0.55$ and $x / D$

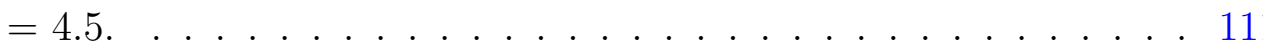

7.7 The variation of the conditional mean mass fraction with $\zeta$ for major and minor species for flame PM1-200 at $\tilde{\theta}=0.55$ and $x / D$

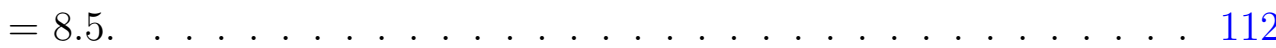

7.8 The variation of the conditional mean mass fraction with $\zeta$ for major and minor species for flame PM1-200 at $\tilde{\theta}=0.55$ and $x / D$ $=10.5 \ldots \ldots \ldots \ldots \ldots \ldots \ldots \ldots \ldots \ldots \ldots \ldots \ldots$

7.9 The typical variation of the conditional scalar dissipation rate, $\left\langle N_{\theta}^{+} \mid \zeta\right\rangle$, obtained in the CMC calculation for flames PM1-50 and PM1-200 at $\tilde{\theta}=0.55, x / D=4.5$. The values are non-dimensionalised using $S_{L}^{o}$ and $\delta_{L}^{o} \ldots \ldots \ldots \ldots \ldots \ldots \ldots \ldots \ldots \ldots$

7.10 The typical variation of the conditional scalar dissipation rate, $\left\langle N_{\theta}^{+} \mid \zeta\right\rangle$, obtained in the CMC calculation for flames PM1-50 and PM1-200 at $\tilde{\theta}=0.55, x / D=8.5$. The values are non-dimensionalised using $S_{L}^{o}$ and $\delta_{L}^{o} \ldots \ldots \ldots \ldots \ldots \ldots \ldots \ldots \ldots$ 
7.11 The typical variation of the conditional scalar dissipation rate, $\left\langle N_{\theta}^{+} \mid \zeta\right\rangle$, obtained in the CMC calculation for flames PM1-50 and PM1-200 at $\tilde{\theta}=0.55, x / D=10.5$. The values are non-dimensionalised using $S_{L}^{o}$ and $\delta_{L}^{o} \ldots \ldots \ldots \ldots \ldots \ldots \ldots \ldots \ldots$

7.12 The variation of the Favre PDF of the progress variable, $\tilde{p}(\zeta)$, at selected locations of the flame brush of flame PM1-50. . . . . . . 115

7.13 The variation of the Favre $\mathrm{PDF}$ of the progress variable, $\tilde{p}(\zeta)$, at selected locations of the flame brush of flame PM1-200. . . . . . . 116

7.14 Computed radial variation of $\widetilde{Z 1}$ from the hot flow simulation at six axial locations for the conditions for flame PM1-200. . . . . . 117

7.15 Computed radial variation of $\widetilde{Z 1}$ from the hot flow simulation are compared to PDF calculations [5] for flame PM1-200. . . . . . . . 118

7.16 Computed radial variation of $\widetilde{Z 2}$ from the hot flow simulation at six axial locations for the conditions for flame PM1-200. . . . . . 118

7.17 Computed radial variation of $\widetilde{Z 3}$ from the hot flow simulation at six axial locations for the conditions for flame PM1-200. . . . . . 119

7.18 Contours of $\widetilde{Z}_{3}$ for flame PM1-200 . . . . . . . . . . . . . . . . 119

7.19 Contours of $\widetilde{Z_{1}}$ and $\widetilde{Z_{2}}$ for flame PM1-200 . . . . . . . . . . . . 120

7.20 Contours of the mean progress variable and its variance for flame PM1-200 . . . . . . . . . . . . . . . . 121

7.21 The mean velocity from the CMC calculations at six axial locations are compared to the experimental data [4] and PDF calculations, $k-\varepsilon$ and $k-\omega[6]$ for flame PM1-200. . . . . . . . . . . . . . . . . 125

7.22 The mean velocity from the CMC calculations at four axial locations are compared to the experimental data [4] and PDF calculations, $k-\varepsilon$ and $k-\omega[6]$ for flame PM1-50. . . . . . . . . . . . . . 126

7.23 The mean rms velocity from the CMC calculations at six axial locations are compared to the experimental data [4] and PDF calculations, $k-\varepsilon$ and $k-\omega[6]$ for flame PM1-200. . . . . . . . . . 127

7.24 The mean rms velocity from the CMC calculations at four axial locations are compared to the experimental data [4] and PDF calculations, $k-\varepsilon$ and $k-\omega[6]$ for flame PM1-50. 
7.25 The mean temperature from the CMC calculations at four axial locations are compared to the experimental data [4] and PDF calculations, $k-\varepsilon$ and $k-\omega$ [6] and VFJPDF [5] for flame PM1-200. . . 129

7.26 The mean temperature from the CMC calculations at four axial locations are compared to the experimental data [4] and PDF calculations, $k-\varepsilon$ and $k-\omega$ [6] and VFJPDF [5] for flame PM1-50. . . 130

7.27 The mean $\widetilde{\mathrm{CO}} \times 10^{3}$ from the CMC calculations at four axial locations are compared to the experimental data [4] and PDF calculations, $k-\varepsilon$ and $k-\omega[6]$ and VFJPDF [5] for flame PM1-200. . . . . 131

7.28 The calculated mean $\widetilde{\mathrm{CO}} \times 10^{3}$ mass fraction from $\mathrm{CMC}$ calculations at four axial locations are compared to the experimental data [4] and PDF calculations, $k-\varepsilon$ and $k-\omega$ [6] and VFJPDF [5] for flame PM1-50. . . . . . . . . . . . . . . . . . 132

7.29 The calculated mean $\overline{\mathrm{OH}} \times 10^{6}$ from CMC calculations at five axial locations are compared to the experimental data [4] and PDF calculations, $k-\varepsilon$ and $k-\omega[6]$ for flame PM1-200. . . . . . . . . . . 133

7.30 The calculated mean $\overline{\mathrm{OH}} \times 10^{6}$ from $\mathrm{CMC}$ calculations at four axial locations are compared to the experimental data [4] and PDF calculations, $k-\varepsilon$ and $k-\omega$ [6] for flame PM1-50. . . . . . . . . . 134 


\section{Nomenclature}

\section{Latin}

$\begin{array}{ll}A & \text { frequency factor } \\ c & \text { progress variable } \\ \bar{c} & \text { Reynolds averaged } c \\ \tilde{c} & \text { Favre averaged } c \\ c^{\prime \prime} & \text { Favre fluctuation of } c \\ C_{\epsilon 1} & \text { constant in } k-\varepsilon \text { model } \\ C_{3}, C_{4} & \text { model parameters, Eq. (4.1) } \\ D & \text { molecular diffusivity } \\ E_{a} & \text { activation energy } \\ h & \text { specific enthalpy } \\ h_{\alpha} & \text { total enthalpy of species } \alpha \\ h^{s} & \text { sensible enthalpy } \\ \tilde{k} & \text { Favre average turbulent kinetic energy } \\ K_{c}^{*} & \text { constants in Eq. (4.1) } \\ N & \text { scalar dissipation rate } \\ \tilde{p}(c) & \text { Favre PDF of } c \\ P & \text { pressure } \\ Q & \text { conditional average } \\ R & \text { characteristics gas constant } \\ \mathcal{R} & \text { universal gas constant }=8.314 \mathrm{JK}^{-1} \mathrm{~mol}^{-1}\end{array}$




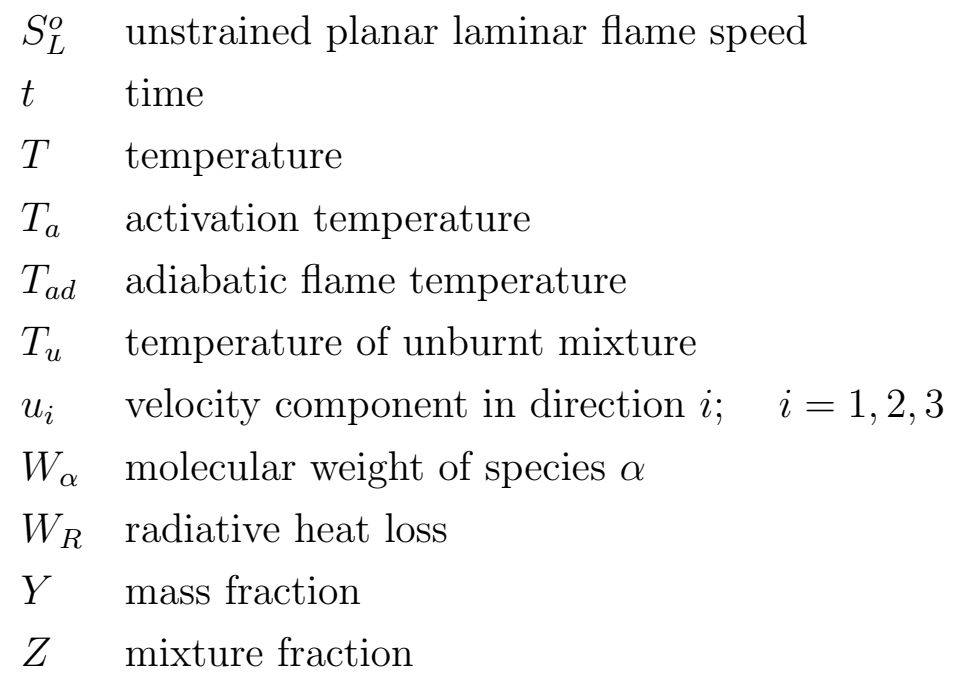

\section{Greek}

$\alpha \quad$ thermal diffusivity, a species in the mixture

$\beta^{\prime} \quad$ model constant

$\delta_{i j} \quad$ Kronecker delta; $\delta_{i j}=1$ if $i=j$ and 0 otherwise

$\delta_{L}^{\circ} \quad$ thermal laminar thickness

$\Delta x \quad$ grid spacing

$\epsilon \quad$ turbulence eddy viscosity

$\tilde{\epsilon} \quad$ Favre average turbulent kinetic energy dissipation rate

$\eta_{k} \quad$ Kolmoforov length scale

$\Lambda \quad$ integral length scale

$\lambda \quad$ thermal conductivity

$\mu \quad$ dynamic viscosity

$\mu_{t} \quad$ turbulent viscosity

$\nu \quad$ kinematic viscosity, stoichiometric coefficient

$\overline{\dot{\omega}} \quad$ mean reaction rate

$\phi \quad$ equivalence ration

$\rho \quad$ density of mixture

$\Sigma \quad$ flame surface density

$\sigma_{i j} \quad$ stress tensor

$\tau \quad$ heat release parameters 


$$
\begin{array}{ll}
\tau_{i j} & \text { shear stress } \\
\tau_{c} & \text { chemical time scale } \\
\tau_{k} & \text { Kolmogrov time scale } \\
\tau_{t} & \text { integral time scale } \\
\tilde{\epsilon_{c}} & \text { Favre averaged scalar dissipation rate of } c \\
\zeta & \text { sample space variable for } c
\end{array}
$$

\section{Symbols}

$\bar{\phi} \quad$ Reynolds average of $\phi$

$\tilde{\phi} \quad$ Favre (density weighted) average of $\phi$

$\phi^{\prime} \quad$ fluctuating component, $\phi^{\prime}=\phi-\tilde{\phi}$

$\phi^{\prime \prime} \quad$ conditional fluctuation, $\phi^{\prime \prime}=\phi-\langle\phi \mid \eta\rangle$

$\widetilde{\phi^{\prime \prime 2}}$ Favre variance of $\phi$

\section{Dimensionless Numbers}

$\begin{array}{lll}D a & \tau_{t} / \tau_{c} & \text { Damköhler number } \\ \text { Le } & S_{C} / P_{r}=\lambda /(\rho c p D) & \text { Lewis number } \\ \operatorname{Pr} & \mu c p / \lambda & \text { Prandtl number } \\ S c & \mu /(\rho D) & \text { Schmidt number } \\ \operatorname{Re} & \rho U L / \mu & \text { Reynolds number } \\ \operatorname{Pe} & \operatorname{Re} \cdot \operatorname{Pr} & \text { Peclet number }\end{array}$

\section{Subscripts}

1 Oxidant stream

$2 \quad$ Fuel stream

$f \quad$ Fuel

$u \quad$ Unburnt mixture

$t \quad$ Turbulent 


\section{Acronyms}

BML Bray-Moss-Libby

CFD Computational Fluid Dynamics

CMC Conditional Moment Closure

CSDR Conditional Scalar Dissipation Rate

DNS Direct Numerical Simulations

EBU Eddy Break-Up

LES Large Eddy Simulation

LHS Left Hand Side

PDF Probability Density Function

RANS Averaged Navier Stokes Simulation

RHS Right Hand Side

RPV Reaction Progress Variable

RSM Reynolds Stress Models 


\section{Chapter 1}

\section{Introduction}

Energy dates back to the beginning of the first human. Primitive man used the wood in the fire for the purpose of heating and cooking. In the later stages, he domesticated animals to help him in agriculture and transport, as well as using other types of energy such as wind and water power for the purpose of maritime transport and irrigation.

Western European countries have witnessed, during the eighteenth and nineteenth centuries, the emergence of the industrial revolution, which shortly thereafter spread around the globe. As a consequence, scientific and technical changes have emerged, especially in industry and transportation sectors. The actual start of the industrial revolution with the invention of the steam engine by James Watt, in 1769 at the University of Glasgow. The basic idea was to transform the steam thermal energy to mechanical energy using coal as a source of energy. Steam engines were used in pumping water and powering trains and ships. The invention of electricity, by Thomas Edison in 1880, followed, by the invention of internal combustion engine, by Rudolf Diesel in 1898, was the start to the shift to the use of fossil fuel in its current forms. Later on, the rapid development in industry 
and transportation sectors increased the demand for fuel.

Fossil fuels are not renewable energy sources and have an impact on the environment and mankind. Combustion can significantly disturb the natural carbon cycle between the earth and atmosphere [7]. For example, the major products from combustion are carbon dioxide $\left(\mathrm{CO}_{2}\right)$ and water $\left(\mathrm{H}_{2} \mathrm{O}\right)$, these gases are known as greenhouse gases. They trap the heat in the atmosphere and as result of that, the average global temperature of earth rises. This phenomenon is widely known as global warming [8]. Also, primarily pollutant such as sulfur dioxide $\left(\mathrm{SO}_{2}\right)$ and nitric oxides $\left(\mathrm{NO}_{\mathrm{x}}\right)$ emitted by the use of fossil fuels, would often reach the upper levels of the atmosphere and drift into areas where natural rain clouds are regularly formed and fall to the ground in the form of acid rains. Acid rain causes severe damage to the environment and humans [9]. As a consequence, the environmental legislation became more stringent to minimise the environmental impact of combustion. For instance, the Clean Air Act 1956 which was introduced after London Smog in 1952. Later on, in 1993, the Charter was updated in its current version to protect the general public from exposure to airborne contaminants that are known to be hazardous to human health.

Although, rising oil prices and growing awareness of the global warming in recent years has led to increase interest in bio-fuel and other forms of noncombustible renewable energy such as solar, wind and hydro power. However, according to European Commission statistics, renewable energy sources accounted for $8.4 \%$ of the EUs consumption in 2008 [10]. Certainly, it is highly unlikely to replace fossil fuel with renewable energy in the near future. Thus, the challenge remains how to reduce emissions and their impact without compromising the efficiency of combustion systems. This is can be achieved by developing new 
combustion technologies that meet environmental and efficiency demands. To accomplish this we must first understand the complex physics of the combustion process.

Combustion is traditionally classified into non-premixed and premixed combustion. In non-premixed combustion, the fuel and the oxidiser enter the combustion zone from two separate streams, but in the premixed situation, the fuel and oxidiser are mixed homogeneously before entering into the combustion zone. However, in practice neither of these two modes occur on their own, but a combination of them is common. The fuel lean premixed combustion is known to have potential to meet the environmental demands without compromising the efficiency by reducing combustion temperature $[11 ; 12 ; 13 ; 14 ; 15 ; 16 ; 17]$. However, the fuel lean premixed flames are susceptible to combustion oscillations, stability and extinction issues. For instance, lean premixed combustion is sensitive to the quality of the mixing in unburned mixture. If the unburned mixture is not perfectly mixed some regions will be richer than others and form high temperature pockets which leads to $\mathrm{NO}_{\mathrm{x}}$ formation [18]. Further more, the stability of lean premixed flames can reduce the efficiency and the life time of the combustion device [19]. Thus, the original equipment manufacturers of combustion systems are in constant search to develop methods, which could be used to identify avenues to improve the stability of turbulent lean premixed flames.

Computational fluid dynamics (CFD), is embraced more in industries to identify potential ways to achieve the desired behaviour of combustion systems, since it is cost-effective and can produce detailed information that are some times difficult and expensive to obtain from experiments. Although modelling of turbulent combustion has been pursued for many years, a precise theoretical description to 
make the combustion models rigorous for industrial use is still evolving due to the complexity of this subject [20]. In premixed flames, there are strong interactions among turbulence and chemical reaction. Turbulence contains a wide range of length and time scales [21]. The chemical reaction process also contains a wide range of length and time scales and determining them over all reaction rates is not a straight forward task for turbulent flames [22]. Thus, modelling turbulent lean premixed flames is challenging. However, combustion models which are capable to address these interactions accurately with ability to predict emissions are required in the design and development of next generation of lean combustion systems. This requirement gives motivation for this study.

Turbulent flows are simulated using three approaches [22; 23]; direct numerical simulation (DNS), large-eddy simulation (LES) and Reynolds averaged Navier stokes simulation (RANS). In DNS, all the length and time scales for turbulence and chemical reactions are captured and the conservation equations (discussed in the next chapter) are solved without any modelling using highly accurate numerical methods. This method is prohibitively expensive to simulate practical flames, because of the geometrical complexities and the range of scales involved. Thus, this method is mainly used as a research tool to gain fundamental insights from turbulent combustion in simple geometries or flows. In LES, the energy containing scales of turbulence are captured explicitly using approximate governing equations. Since chemical reactions occur at small scales, some sort of models are required to represent combustion and its interaction with turbulence. These models are known [22] as sub-grid scale combustion models. However, the numerical resolution requirement for LES and the uncertainty in the sub-grid scale modelling currently limits the use of LES for industry scale flames. 
In RANS, described in some details in chapter 2, all the scales of turbulence and reactions are averaged and thus, the computational costs are lower compared to the previous two approaches. However, the interaction of scales and their effects are to be included using turbulence and combustion models, discussed in the next chapter. Since this method is developed in the past and many computational tools are readily available, the use of RANS is more common in industries, but this may change in the next decade or so. However, as noted earlier, the currently available combustion models are not as good as one would like to have although RANS simulation is commonly used to model the turbulent reacting flows since it is computationally cheaper approach compared to DNS and LES.

The main objective of turbulent combustion modelling is to provide a closure for the mean reaction rate, $\overline{\dot{\omega}}_{\alpha}$, which appears in the mean species transport equation. The reaction rate is a highly non-linear function of temperature and species concentration, evaluating its mean value using average temperature and scalar concentrations is known to be erroneous [24]. Flamelet based methods are common for turbulent premixed flames [17] and these methods consider the turbulent flame as a collection of laminar flames. This view is acceptable if the flame scales are much smaller than the turbulence scales and thus the laminar flame structure is not disturbed by turbulence. Even if the turbulence scales become smaller than the thermal thickness of the laminar flame, the flamelet concept can be used if the reaction zone thickness is smaller than the turbulence scale. However, if the reaction time scale for a scalar become larger than the typical time scale for smaller turbulence eddies then the use of flamelet based ideas to compute that particular scalar becomes an issues $[25 ; 26]$. Pollutants such as carbon monoxide, nitric oxides are good examples for this. Alternative methods 
such as conditional moment closure (CMC) [27; 28] and transported probability density function (transported PDF) [29] have been proposed and developed. The $\mathrm{CMC}$, described in chapter 3 is of specific interest to this study for the following reason. Although the CMC has been developed and successfully applied to various non-premixed combustion systems such as hood fires [30], lifted flames [31], bagasse-fired boiler [32], bluff-body flames [33; 34], spray ignition [35] and soot formation [36], its application to premixed flames is not fully tested and validated yet.

\section{$1.1 \quad$ Objectives}

The prime objective of this work is to study if the pollutants emitted from lean premixed combustion can be calculated using $\mathrm{CMC}$ and to validate this calculations using published experimental results.

The specific objectives are:

1. To further develop CMC sub-models for premixed combustion.

2. To develop a new model for the conditional scalar dissipation rate required in the $\mathrm{CMC}$ for premixed flames.

3. To validate the performance of this model with DNS data.

4. To implement the CMC sub models for premixed flames into a computational code.

5. To validate the simulation results using two different experimental data sets $[2 ; 3]$. 
The outline of this thesis is as follows.

Chapter 2 presents a brief review of the theory and background of turbulent premixed flames. Chapter 3 describes the CMC methodology for turbulent premixed flames and chapter 4 discuses the modelling of mean and conditional scalar dissipation rates used in this study. Chapter 5 describes the numerical methods and the computational tool used in this study. The chosen test cases are described briefly in chapters 6 and 7 along with the simulation results. The conclusions of this study are summarised in the final chapter along with some suggestion for future directions for the CMC modelling of turbulent premixed flames. 


\section{Chapter 2}

\section{Background on Turbulent Premixed Combustion}

The scope of this chapter is to present a comprehensive but a concise, overview of turbulent premixed combustion modelling that appears in the literature $[16 ; 22$; $23 ; 25 ; 26 ; 37 ; 38 ; 39]$. The well known modes of combustion are; non-premixed and premixed combustion, the second type is the focus of this study, as noted in chapter 1.

Non-premixed flames are also called diffusion flames, the oxidiser and the fuel streams are completely separated as shown in Fig 2.1. The fuel and oxidiser diffuse simultaneously towards the reaction zone by molecular diffusion to sustain the combustion. The classical examples for non-premixed combustion include the compression ignition engines, furnaces and fires.

In premixed combustion the fuel and oxidiser are homogeneously mixed in a desired proportion prior to combustion and a suitable ignition source, e.g. spark, provides sufficient energy needed to initiate combustion. Exothermic reactions 


\section{Background on Turbulent Premixed Combustion}

release energy in the flame front which propagates normal to itself to consume the available reactant mixture with a flame propagation speed $S_{L}^{\circ}$. The burnt gases and fresh gases are separated by a thin flame with a thickness, $\delta_{L}^{\circ}$, of about $0.5 \mathrm{~mm}$. The structure of a laminar premixed flame is presented in Fig 2.2. However, in practical combustion devices the combustion occurs in mixed mode having characteristics of both non-premixed and premixed combustion due to incomplete mixing between fuel and oxidiser causing large local fluctuations in the equivalence ratio. This mode of combustion is called partially premixed combustion [26].

The occurrence of turbulence in practical combustors is inevitable and thus one must consider turbulent combustion in modelling. The major challenge in turbulent combustion modelling is due to the interaction of turbulence and chemical reactions. This has been studied in the past and these studies, specifically on turbulent premixed flames, are reviewed briefly in the rest of this chapter.

This chapter is organised as follows. Since the governing equations are the starting point for the modelling studies, the instantaneous conservation equations for mass, momentum, energy and species mass fractions are discussed in section 2.1 along with other constitutive relations required. The modelling of instantaneous reaction rate is noted briefly in section 2.1.2. The three simulation paradigms are briefly discussed to present their essential features, advantages and disadvantages in section 2.2. Since RANS methodology is used in this study, this methodology and related turbulence modelling are discussed in sections 2.3 and 2.4 respectively in some detail. The background studies on turbulent premixed combustion and its modelling are discussed in section 2.5. 


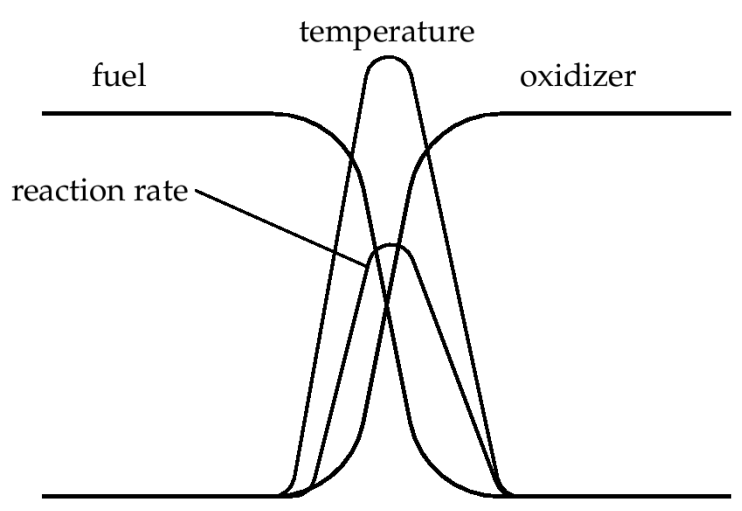

Figure 2.1: Structure of a laminar diffusion flames.

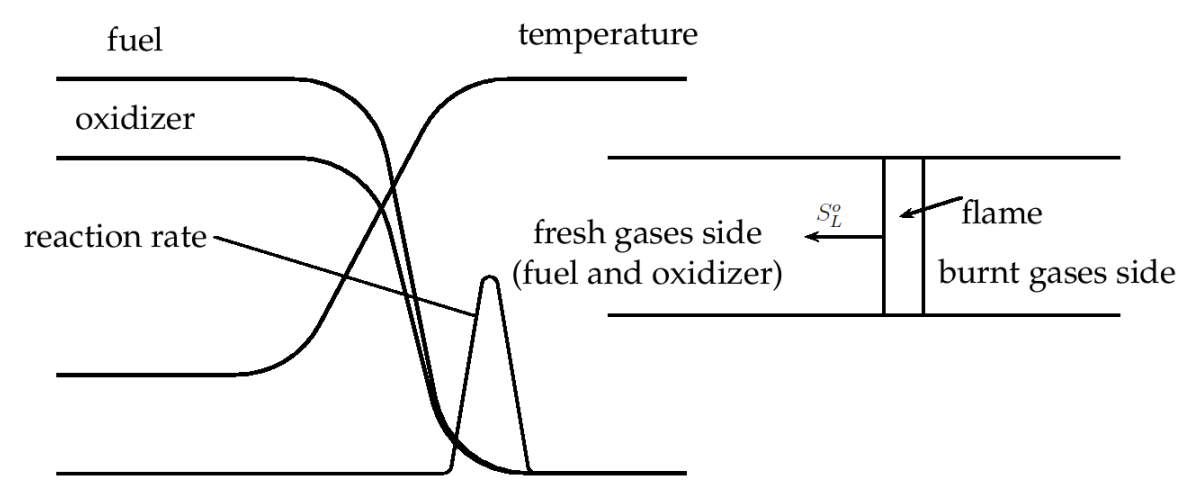

Figure 2.2: Structure of a laminar premixed flames.

\subsection{Governing Equations}

\subsubsection{Fluid Flow Equations}

These equations are given by the conservation of mass, momentum, species mass fractions and energy in Cartesian coordinates of a reacting system with $N$ species and $I$ reactions $[22 ; 23 ; 37 ; 38]$.

- Conservation of mass 


$$
\frac{\partial \rho}{\partial t}+\frac{\partial\left(\rho u_{i}\right)}{\partial x_{i}}=0
$$

The first and second terms on the LHS of Eq. (2.1) are local temporal change of $\rho$ and its convective transport respectively inside a control volume.

- Conservation of momentum

$$
\frac{\partial\left(\rho u_{i}\right)}{\partial t}+\frac{\partial\left(\rho u_{i} u_{j}\right)}{\partial x_{j}}=-\frac{\partial p}{\partial x_{i}}+\frac{\partial \tau_{i j}}{\partial x_{j}}+F_{i}
$$

The first and second terms on LHS of Eq. (2.2) are the unsteady and advective terms respectively. The first and third terms on the RHS represent the contribution of the pressure gradient and body force in the $i$ th coordinate direction respectively to the force balance. The second term on the RHS is due to the viscous forces, where $\tau_{i j}$ denotes the viscous stress tensor,

$$
\tau_{i j}=\mu\left(\frac{\partial u_{i}}{\partial x_{j}}+\frac{\partial u_{j}}{\partial x_{i}}\right)-\frac{2}{3} \mu \frac{\partial u_{k}}{\partial x_{k}} \delta_{i j}
$$

where $\mu$ is the dynamic viscosity and $\delta_{i j}$ is the Kronecker delta.

- Conservation of mass fraction of species $\alpha$

$$
\frac{\partial\left(\rho Y_{\alpha}\right)}{\partial t}+\frac{\partial\left(\rho u_{i} Y_{\alpha}\right)}{\partial x_{i}}=-\frac{\partial J_{i}^{\alpha}}{\partial x_{i}}+\dot{\omega}_{\alpha} \quad(\alpha=1,2, \ldots, N)
$$




\section{Background on Turbulent Premixed Combustion}

The first and second terms on the LHS of Eq. (2.4) are local rate of change of $Y_{\alpha}$ and convective transport respectively. The first and second terms on the RHS are molecular diffusion flux of species $\alpha$ in the $i$ th coordinate direction and the total rate of mass production by chemical reaction respectively. The diffusion flux is represented by Fick's first law as

$$
\frac{\partial J_{i}^{\alpha}}{\partial x_{i}}=-\frac{\partial}{\partial x_{i}}\left(\rho D_{\alpha} \frac{\partial Y_{\alpha}}{\partial x_{i}}\right)=-\frac{\partial}{\partial x_{i}}\left(\frac{\mu}{S c_{\alpha}} \frac{\partial Y_{\alpha}}{\partial x_{i}}\right)
$$

where $D_{\alpha}$ is the molecular diffusivity of species $\alpha$ relative to other species and $S c_{\alpha}$ is the Schmidt number of species $\alpha$.

- Conservation of energy

The enthalpy, $h$, of a reactive mixture is defined as the sum of the specific enthalpies $h_{\alpha}$ of species $\alpha$

$$
h=\sum_{\alpha=1}^{n} Y_{\alpha} h_{\alpha} .
$$

The absolute enthalpy, $h_{\alpha}$, is equal to the sum of enthalpy of formation, $h_{f, \alpha}^{\circ}$, of species $\alpha$ at a reference temperature and sensible enthalpy, $h^{s}$, so that

$$
h_{\alpha}=h_{f, \alpha}^{\circ}+h_{\alpha}^{s} .
$$


The sensible enthalpy, $h^{s}$, of an ideal gas is equal to

$$
h_{\alpha}^{s}=\int_{T_{r e f}}^{T} c_{p_{\alpha}}(T) d T,
$$

where $T$ is the absolute temperature. By assuming low Mach number flow, the simple form of the energy conservation equation can be written as [22]

$$
\frac{\partial(\rho h)}{\partial t}+\frac{\partial\left(\rho u_{i} h\right)}{\partial x_{i}}=\frac{\partial p}{\partial t}+\frac{\partial}{\partial x_{i}}\left(\frac{\lambda}{c_{p}} \frac{\partial h}{\partial x_{i}}\right)+S_{R}
$$

The two terms on the LHS have their usual meaning. The first term on the RHS represents the pressure gradient while the second and third terms represent the diffusion of enthalpy and the source or sink term due to radiative heat exchange respectively.

- State equation

The pressure, $p$, is obtained from the equation of state as

$$
p=\rho \mathcal{R} T \sum_{\alpha=1}^{n} \frac{Y_{\alpha}}{W_{\alpha}} .
$$

It is common to use two additional equations, the reaction progress variable and mixture fraction equations in turbulent combustion modelling and simulation studies. These two equations are presented next.

- Progress variable

The progress variable, $c$, is required for turbulent premixed combustion modelling which measures the reaction progress in premixed combustion. The progress 
variable can be defined based on temperature, $T$, or using fuel mass fraction, $Y_{f}$, [17] as

$$
C_{T}=\frac{T-T_{u}}{T_{b}-T_{u}}
$$

where $T_{u}$ and $T_{b}$ respectively denote the temperature of unburnt and burnt mixture in adiabatic laminar flames, and

$$
C_{f}=1-\frac{Y_{f}}{Y_{f}^{u}} .
$$

If the Lewis numbers, define as the ratio of thermal diffusivity to mass diffusivity, of the mixture is unity then $C_{f}=C_{T}$ [22]. An alternative choice using the sensible enthalpy is also possible [40]:

$$
c=\frac{h^{s}-h_{u}^{s}}{h_{b}^{s}-h_{u}^{s}}
$$

The subscripts $b$ and $u$ denote the burnt and unburnt mixtures respectively. For this study, $c$ based on fuel mass fraction is used and it is governed by

$$
\frac{\partial(\rho c)}{\partial t}+\frac{\partial\left(\rho u_{i} c\right)}{\partial x_{i}}-\frac{\partial}{\partial x_{i}}\left(\rho D_{c} \frac{\partial c}{\partial x_{i}}\right)=\rho \dot{\omega}_{c}
$$

in the usual nomenclature.

- Mixture fraction

The mixture fraction is a passive scalar which describes the stoichiometry of the reacting mixture. It takes a value of 0 in the oxidiser stream and 1 in the fuel 
stream. The instantaneous transport equation for $Z$ is given by

$$
\frac{\partial(\rho Z)}{\partial t}+\frac{\partial\left(\rho u_{i} Z\right)}{\partial x_{i}}-\frac{\partial}{\partial x_{i}}\left(\rho D_{Z} \frac{\partial Z}{\partial x_{i}}\right)=0
$$

\subsubsection{Reaction Rates}

Consider a set of $I$ elementary reactions, which can be expressed in symbolic form as

$$
\sum_{\alpha=1}^{N} \nu_{\alpha}^{\prime} \mathcal{M}_{\alpha} \Longleftrightarrow \sum_{\alpha=1}^{N} \nu_{\alpha}^{\prime \prime} \mathcal{M}_{\alpha}
$$

The net rate of chemical reaction $k$ is given by

$$
\dot{\omega}_{k}=k_{f k} \prod_{\alpha=1}^{N}\left(\frac{\rho Y_{\alpha}}{W_{\alpha}}\right)^{\nu_{\alpha k}^{\prime}}-k_{b k} \prod_{\alpha=1}^{N}\left(\frac{\rho Y_{\alpha}}{W_{\alpha}}\right)^{\nu_{\alpha k}^{\prime \prime}}
$$

where $k_{f k}$ and $k_{b k}$ are the forward and backward rate coefficients respectively and $W_{\alpha}$ is the molecular weight of species $\alpha$. The exponent $\nu_{\alpha k}^{\prime}$ and $\nu_{\alpha k}^{\prime \prime}$ denote the forward and backward stoichiometric coefficients respectively. The forward and backward rate coefficients are expressed using Arrhenius form as

$$
k=A T^{n} \exp \left(-E_{a} / \mathcal{R} T\right)
$$

where $A$ is the pre-exponential factor and $E_{a}$ is the activation energy. It should also be noted that the backward rate is related to the forward rate through the equilibrium constant. Now, the chemical reaction rate, on a mass basis, for species $\alpha$ is given by 


$$
\dot{\omega}_{\alpha}=W_{\alpha} \sum_{k=1}^{I} \dot{\omega}_{k} \quad\left(\nu_{\alpha k}^{\prime \prime}-\nu_{\alpha k}^{\prime}\right) .
$$

\subsection{Three Simulation Paradigms}

In the simulation of turbulent premixed flames, the above conservation equations are solved with or without additional models depending on the methodology to be followed. As noted in chapter 1, there are three methods used commonly, and these methods are DNS, LES and RANS. The essential features, advantages and disadvantages of these methodologies are briefly discussed below.

\subsubsection{DNS}

In direct numerical simulation, the full set of the instantaneous Navier-Stokes equations discussed in section 2.1 are solved without any turbulence models. It resolves all spatial and temporal scales in turbulent flows and simulates the problem in detail with specified initial and boundary conditions. Thus, DNS can produce detailed information which may not be available from experimental measurements due to the cost involved.

However, unfortunately there are restrictions in applying the method to realistic turbulent flows, due to the non-linearity in the governing equations and the wide range of length and time scales in such flows [16]. For instance, to resolve all the spatial and temporal length scales, the integral length $\Lambda$ and Kolmogorov length $\eta_{k}$ scales must be captured by the simulation grid. This is can be achieved 


\section{Background on Turbulent Premixed Combustion}

only when

$$
L=N . \Delta x \geqslant \Lambda \quad \text { and } \quad \Delta x=\frac{L}{N} \leqslant \eta_{k},
$$

where $L$ is the domain size, $\Delta x$ is the grid spacing and $N$ is the number of grid points in each direction in the physical space. Since the turbulent Reynolds number, $R e_{t}$, is related to the turbulent length scales by [22]

$$
R e_{t}^{3 / 4}=\frac{\Lambda}{\eta_{k}},
$$

then the maximum number of grid points used in DNS simulation must obey $N>R e_{t}^{3 / 4}[22]$. Therefore, the DNS simulation is prohibitively expensive for realistic flows with Reynolds number of order of $10^{6}[16]$.

For turbulent reacting flows this situation is even more complex, because chemical reactions introduce further length and timescales. In some cases, these scales are smaller then the turbulence scales. This situation requires further resolution to resolve all the range of the scales involved in the combustion process. Therefore, DNS is used only in laboratory scale for research purposes to study the physics of combustion [41; 42; 43], or for a flow with low Reynolds number [44]. The chemistry used in DNS, has an influence on the complexity of the DNS by increasing the number of differential equations needed to be solved. Thus, DNS of turbulent combustion is simplified by using a single global chemical reaction or limited sets of elementary reactions. 


\subsubsection{LES}

LES is considered to be an intermediate tool between DNS and RANS. In this approach, only the large scale eddies are resolved using the filtered form of the governing equations shown in section 2.1, while the effect of small scales eddies, which are the most expensive computational wise, are represented by models $[45 ; 46 ; 47 ; 48 ; 49]$. This technique reduces the restriction in the domain size and the computational expenses in DNS simulation that makes LES applications to practical systems possible. The filter retains eddies with lengths greater than the filter width, $\Delta$, to be resolved on the grid such that $\Delta x \approx \Delta$. On the other hand, the small scales are removed and modelled by sub-grid scales (SGS). The filtered quantity $\phi$ is defined as

$$
\bar{\phi}(\mathbf{x}, t)=\int \phi(\mathbf{x}, t) F\left[\left(\mathbf{x}-\mathbf{x}^{\prime}\right) ; \Delta\right] d x^{\prime}
$$

where $F$ is the filtering function and it can have a different prescribed shapes [22]. The values of $F$ is close to zero when $x-x^{\prime}$ exceeds the filter size $\Delta$.

\subsubsection{RANS}

Since this approach is used for this study, the next section describes this method in some detail.

\subsection{RANS Methodology}

In this method, the instantaneous governing equations are averaged and then solved. Since the governing equations are non-linear, the averaging process in- 


\section{Background on Turbulent Premixed Combustion}

troduces additional unknown terms. This can be seen clearly in the following example. By decomposing an instantaneous quantity $\phi$ as its mean and fluctuating parts, $\phi(\mathbf{x}, t)=\bar{\phi}(\mathbf{x}, t)+\phi^{\prime}(\mathbf{x}, t)$, the mass conservation equation gives

- Conservation of Mass

$$
\frac{\partial \bar{\rho}}{\partial t}+\frac{\partial}{\partial x_{i}}\left(\overline{\rho u_{i}}+\overline{\rho^{\prime} u_{i}^{\prime}}\right)=0
$$

where the over bar denotes an averaging procedure, which can be either spatial or temporal depending on the flow characteristics. The averaging procedure yields additional term $\left(\overline{\rho^{\prime} u_{i}^{\prime}}\right)$, which arises from the correlations between the velocity and the density fluctuations and it needs to be modelled. To avoid these kind of correlations terms involving density fluctuations, Favre or density weighted averaging is used for flows involving large density variation, such as combustion. The instantaneous quantity is decomposed as $\phi(\mathbf{x}, t)=\widetilde{\phi}(\mathbf{x}, t)+\phi^{\prime \prime}(\mathbf{x}, t)$, where $\widetilde{\phi}=\overline{\rho \phi} / \bar{\rho}$ and $\widetilde{\phi^{\prime \prime}}=0$. It is well known that applying Favre averaging to the governing equations in section 2.1, gives additional terms, such as $\widetilde{u_{i}^{\prime \prime} u_{j}^{\prime \prime}}, \widetilde{u_{i}^{\prime \prime} Y_{\alpha}^{\prime \prime}}$, etc. Applying Favre averaging to the instantaneous governing equations gives

- conservation of mass

$$
\frac{\partial \bar{\rho}}{\partial t}+\frac{\partial \bar{\rho} \widetilde{u}_{i}}{\partial x_{i}}=0
$$

- conservation of momentum

$$
\frac{\partial\left(\bar{\rho} \widetilde{u}_{i}\right)}{\partial t}+\frac{\partial\left(\bar{\rho} \widetilde{u_{i}} \widetilde{u}_{j}\right)}{\partial x_{i}}=-\frac{\partial \bar{p}}{\partial x_{j}}+\frac{\partial}{\partial x_{i}}\left(\tau_{i j}-\overline{\rho u_{i}^{\prime \prime} u_{j}^{\prime \prime}}\right)+\overline{F_{i}},
$$




\section{Background on Turbulent Premixed Combustion}

where, $\overline{\rho u_{i}^{\prime \prime} u_{j}^{\prime \prime}}$ is the Reynolds stress and its closure is discussed in section 2.4.

- conservation of mass fraction of species $\alpha$

$$
\frac{\partial\left(\bar{\rho} \widetilde{Y_{\alpha}}\right)}{\partial t}+\frac{\partial\left(\bar{\rho} \widetilde{u_{i}} \widetilde{Y_{\alpha}}\right)}{\partial x_{i}}=\frac{\partial}{\partial x_{i}}\left(\overline{\rho D_{\alpha} \frac{\partial Y_{\alpha}}{\partial x_{i}}}-\overline{\rho u_{i}^{\prime \prime} Y_{\alpha}^{\prime \prime}}\right)+\overline{\dot{\omega}}_{\alpha} \quad(\alpha=1,2, \ldots, N)
$$

Closures for the turbulent fluxes, $\overline{\rho u_{i}^{\prime \prime} Y_{\alpha}^{\prime \prime}}$, and mean reaction rate, $\overline{\dot{\omega}}_{\alpha}$, are discussed later in sections 2.4 and 2.5 respectively.

- conservation of energy

$$
\frac{\partial(\bar{\rho} \widetilde{h})}{\partial t}+\frac{\partial\left(\bar{\rho} \widetilde{u_{i}} \widetilde{h}\right)}{\partial x_{i}}=\frac{\partial \bar{p}}{\partial t}-\frac{\partial}{\partial x_{i}}\left(\bar{\rho} \widetilde{u_{i}^{\prime \prime} h^{\prime \prime}}\right)+\frac{\partial}{\partial x_{i}}\left(\frac{\lambda}{c_{p}} \frac{\partial \widetilde{h}}{\partial x_{i}}\right) .
$$

- progress variable equation

$$
\frac{\partial(\bar{\rho} \widetilde{c})}{\partial t}+\frac{\partial\left(\bar{\rho} \widetilde{u_{i}} \widetilde{c}\right)}{\partial x_{i}}=\frac{\partial}{\partial x_{i}}\left(\overline{\rho D_{c} \frac{\partial c}{\partial x_{i}}}-\overline{\rho u_{i}^{\prime \prime} c^{\prime \prime}}\right)+\overline{\dot{\omega}}_{c}
$$

A closure for the turbulent scalar flux, $\overline{\rho u_{i}^{\prime \prime} c^{\prime \prime}}$, and the mean reaction rate are discussed later.

- mixture fraction equation

$$
\frac{\partial(\bar{\rho} \widetilde{Z})}{\partial t}+\frac{\partial\left(\bar{\rho} \widetilde{u_{i}} \widetilde{Z}\right)}{\partial x_{i}}=\frac{\partial}{\partial x_{i}}\left(\overline{\rho D_{Z} \frac{\partial Z}{\partial x_{i}}}-\overline{\rho u_{i}^{\prime \prime} Z^{\prime \prime}}\right)
$$




\section{Background on Turbulent Premixed Combustion}

In addition to the governing equations for the mean progress variable and mixture fraction, transport equations for their variances must also be solved. These equations are $[16 ; 22]$

$$
\begin{array}{r}
\frac{\partial\left(\bar{\rho} \widetilde{c^{\prime \prime 2}}\right)}{\partial t}+\frac{\partial\left(\bar{\rho} \widetilde{u_{i}} \widetilde{c^{\prime \prime 2}}\right)}{\partial x_{i}}=\frac{\partial}{\partial x_{i}}\left(\overline{\rho D_{c} \frac{\partial c^{\prime \prime 2}}{\partial x_{i}}}-\overline{\rho u_{i}^{\prime \prime} c^{\prime \prime 2}}\right)-2 \overline{\rho u_{i}^{\prime \prime} c^{\prime \prime}} \frac{\partial \tilde{c}}{\partial x_{i}} \\
-2 \bar{\rho} \tilde{\epsilon_{c}}+2 \overline{c^{\prime \prime} \dot{\omega}_{c}}
\end{array}
$$

and

$$
\begin{array}{r}
\frac{\partial\left(\bar{\rho} \widetilde{Z^{\prime \prime 2}}\right)}{\partial t}+\frac{\partial\left(\bar{\rho} \widetilde{u_{i}} \widetilde{Z^{\prime \prime 2}}\right)}{\partial x_{i}}=\frac{\partial}{\partial x_{i}}\left(\overline{\rho D_{Z} \frac{\partial Z^{\prime \prime 2}}{\partial x_{i}}}-\overline{\rho u_{i}^{\prime \prime} Z^{\prime \prime 2}}\right)-2 \overline{\rho u_{i}^{\prime \prime} Z^{\prime \prime}} \frac{\partial \tilde{Z}}{\partial x_{i}} \\
-2 \bar{\rho} \tilde{\epsilon}_{Z}
\end{array}
$$

The contribution of the turbulent fluxes, dissipation rates and chemical reactions in the above two variance equations are to be modelled. The modelling of turbulent fluxes are discussed in the next section and the modelling of reaction related terms are discussed in chapter 3. The dissipation rate of the mixture fraction variance is given by $\bar{\rho} \widetilde{\epsilon}_{Z}=\overline{\rho D_{Z}\left(\partial Z^{\prime \prime} / \partial x_{i}\right)\left(\partial Z^{\prime \prime} / \partial x_{i}\right)}$ and it is modelled using the classical model [22] given by

$$
\tilde{\epsilon_{Z}} \approx C_{D}\left(\frac{\tilde{\varepsilon}}{\tilde{k}}\right) \widetilde{Z^{\prime \prime 2}}
$$

where $C_{D}$ is a model parameter which takes a value of about 0.9 to 1.0. The modelling of the dissipation rate, $\widetilde{\rho} \widetilde{\epsilon}_{c}=\overline{\rho D_{c}\left(\partial c^{\prime \prime} / \partial x_{i}\right)\left(\partial c^{\prime \prime} / \partial x_{i}\right)}$, is discussed in details in chapter 4 . 


\subsection{Turbulence Models}

\subsubsection{Turbulent Reynolds Stress Model}

The transport equations presented in the previous section are unclosed and models are required for Reynolds stresses, turbulent transport, mean reaction rates, and dissipation rates. In this section, the turbulence modelling is reviewed very briefly and the $k-\varepsilon$ models used in this study for turbulent premixed flames are discussed in some detail $[22 ; 23]$. The Reynolds stress, $\widetilde{\rho} \widetilde{u_{i}^{\prime \prime} u_{j}^{\prime \prime}}$, are modelled using the turbulent viscosity hypothesis, and according to this hypothesis the Reynolds stress is proportional to the mean rate of strain [22; 23]:

$$
\bar{\rho} \widetilde{u_{i}^{\prime \prime} u_{j}^{\prime \prime}}=-\mu_{t}\left(\frac{\partial \widetilde{u_{i}}}{\partial x_{j}}+\frac{\partial \widetilde{u_{j}}}{\partial x_{i}}-\frac{2}{3} \delta_{i j} \frac{\partial \widetilde{u_{k}}}{\partial x_{k}}\right)+\frac{2}{3} \bar{\rho} \tilde{k} \delta_{i j}
$$

involving the turbulent viscosity, $\mu_{t}$, which needs to be modelled. Many approaches [50] have been proposed in the past for this modelling. In the simplest possible approach proposed by Prandtl, the eddy viscosity is given by $\mu_{t}=\bar{\rho} l_{m i x}^{2}|\tilde{S}|$, where $\tilde{S}$ is the mean stress tensor defined as $\tilde{S}_{i j}=\left(\frac{\partial \tilde{u}_{i}}{\partial x_{j}}+\frac{\partial \tilde{u}_{j}}{\partial x_{i}}\right) / 2$, and $l_{m i x}$ is the mixing length. This model is known as mixing length or zero equation model.

In another approach [50], the eddy viscosity is modelled as $\mu_{t}=\bar{\rho} C_{\mu} l_{m} \tilde{k}^{\frac{1}{2}}$, where $\tilde{k}$ is the turbulence kinetic energy obtained from the solution of its transport equation and $C_{\mu}$ is a model parameter.

It is known from many past studies [51] that the eddy viscosity obtained using the solution of $\tilde{k}$ and its dissipation rate, $\tilde{\varepsilon}$, transport equations give an acceptable level of accuracy for a range of flows. This approach, is widely used in RANS 


\section{Background on Turbulent Premixed Combustion}

simulation and is called the $k-\varepsilon$ [51] model. The turbulent viscosity is given by

$$
\mu_{t}=\bar{\rho} C_{\mu}\left(\widetilde{k}^{2} / \widetilde{\varepsilon}\right)
$$

The transport equations for $\tilde{k}$ and $\tilde{\varepsilon}$ are written as [52]

$$
\frac{\partial(\bar{\rho} \widetilde{k})}{\partial t}+\frac{\partial\left(\bar{\rho} \widetilde{u_{i}} \widetilde{k}\right)}{\partial x_{i}}=\frac{\partial}{\partial x_{i}}\left[\left(\mu+\frac{\mu_{t}}{\sigma_{\tilde{k}}}\right) \frac{\partial \widetilde{k}}{\partial x_{i}}\right]+P_{k}-\bar{\rho} \widetilde{\varepsilon}
$$

and

$$
\frac{\partial(\bar{\rho} \widetilde{\varepsilon})}{\partial t}+\frac{\partial\left(\bar{\rho} \widetilde{u_{i}} \widetilde{\varepsilon}\right)}{\partial x_{i}}=\frac{\partial}{\partial x_{i}}\left[\left(\mu+\frac{\mu_{t}}{\sigma_{\varepsilon}}\right) \frac{\partial \widetilde{\varepsilon}}{\partial x_{i}}\right]+C_{\varepsilon 1} \frac{\widetilde{\varepsilon}}{\widetilde{k}} P_{\widetilde{k}}-C_{\varepsilon 2} \frac{\widetilde{\rho} \frac{\widetilde{\varepsilon}^{2}}{\widetilde{k}}}{.}
$$

where the source term $P_{k}$ is given as $P_{k}=-\bar{\rho} \widetilde{u_{i}^{\prime \prime} u_{j}^{\prime \prime}}\left(\partial \widetilde{u_{i}} / \partial x_{i}\right)-\overline{u_{i}^{\prime \prime}} \partial \bar{p} / \partial x_{i}+\overline{p^{\prime} u_{i}^{\prime \prime} / \partial x_{i}}$. The Reynolds stresses, $\widetilde{\rho} \widetilde{u_{i}^{\prime \prime} u_{j}^{\prime \prime}}$, are determined from equation Eq. (2.33) and the standard model constants [16] are $C_{\mu}=0.09, \sigma_{k}=1, \sigma_{\varepsilon}=1.3, C_{\varepsilon 1}=1.44$ and $C_{\varepsilon 2}=1.92$. The pressure related terms are to be modelled and their contributions can be significant for turbulent premixed flames in enclosed geometries [53] and can be ignored for open flames. In this study, these terms are neglected.

In another approach, known as the Reynolds stress method (RSM), transport equations for the individual components of the Reynolds stress are solved with additional models $[54 ; 55 ; 56]$. This model is expected to provide improved accuracy for complex flows but with relatively high computational cost. Also, the Reynolds stress equations are stiff causing difficulties while finding their numerical solution [22]. 


\subsubsection{Turbulent Scalar Flux Model}

To solve the progress variable, $\tilde{c}$, transport equation shown in section 2.1 , the turbulent scalar flux, $\widetilde{u_{i}^{\prime \prime} c^{\prime \prime}}$, needs to be modelled, where the symbols, $u_{i}^{\prime \prime}$ and $c^{\prime \prime}$, are the Favre fluctuation of velocity vector and progress variable, respectively [57]. This term is often modelled using the classical gradient transport hypothesis based on the eddy viscosity [58] given as

$$
\bar{\rho} \widetilde{u_{i}^{\prime \prime} c^{\prime \prime}}=\overline{\rho u_{i}^{\prime \prime} c^{\prime \prime}}=-\frac{\mu_{t}}{S c_{c}} \frac{\partial \widetilde{c}}{\partial x_{i}}
$$

where $\mu_{t}$ is the turbulent eddy viscosity and $S c_{c}$ is the turbulent Schmidt number. According to the gradient assumption, turbulent flux transport is analogues to molecular transport. However, theoretical analysis [59; 60], experimental [61; 62; $63 ; 64 ; 65]$ and DNS $[57 ; 66 ; 67]$ studies pointed out the existence of both gradient and counter gradient fluxes in turbulent premixed flames. The transition from gradient type to counter gradient type is influenced by the ratio $u^{\prime} / S_{L}^{\circ}$ and the heat release factor $\tau$ [66], where $u^{\prime}$ is the rms of turbulence velocity fluctuations and $S_{L}^{\circ}$ is the laminar flame speed. According to the DNS analysis in [66], the counter gradient transport occurs when the flow field is dominated by thermal expansion due to the heat release, while the gradient transport occurs when the flow field is dominated by turbulence, ie., when the ratio $u^{\prime} / S_{L}^{\circ}$ is large. The physical mechanism responsible for counter-gradient transport in turbulent premixed flames is explained by Bray-Moss-Libby model [59], where the turbulent scalar flux is given by

$$
\bar{\rho} \widetilde{u_{i}^{\prime \prime} c^{\prime \prime}}=\bar{\rho} \tilde{c}(1-\tilde{c})\left(\bar{u}_{i b}-\bar{u}_{i u}\right)
$$


where, $\bar{u}_{i b}$ and $\bar{u}_{i u}$ are the conditional mean velocity for burnt and unburnt gases, respectively. Since the density of the burnt gases, $\rho_{b}$, is lower compared to unburnt gases, $\rho_{u}$, thus, the pressure gradient due to the heat release accelerates preferentially more the burnt gases compared to the unburnt gases that makes $\bar{u}_{i b}>\bar{u}_{i u}$. This situation will promote counter gradient flux which contradicts the gradient assumption given in Eq. 2.37. A transport equation for the turbulent flux can be solved to account for the occurrence of gradient and counter gradient fluxes as has been done in [68] and explained in [16]. A simple algebraic model has also been proposed in an earlier study [66] to include the gradient and nongradient scalar flux transports in premixed flames. However, it is not uncommon to use gradient flux model in calculations of high Reynolds number turbulent premixed flames and this model is used in this work.

\subsection{Premixed Combustion Sub-Models}

The main objective of turbulent combustion modelling is to provide models for the mean reaction rate term $\overline{\dot{\omega}}_{\alpha}$ which appears in the species transport equation. The average reaction rate $\overline{\dot{\omega}}_{\alpha}$ cannot be easily expressed as a function of averaged mass fractions $\widetilde{Y_{f}}, \widetilde{Y}_{o}$, mean density $\bar{\rho}$ and mean temperature $\widetilde{T}$ due to the nonlinearity associated with this term. If one assumes a single step reaction then the mean reaction rate can be expressed as [22].

$$
\overline{\dot{\omega}}_{f}=-A \bar{\rho}^{2} \tilde{T}^{n} \tilde{Y}_{f} \tilde{Y}_{o} \exp \left(-\frac{T_{a}}{\tilde{T}}\right)\left[1+\left(\frac{T_{a}}{\tilde{T}}\right)^{2} \frac{\widetilde{T^{\prime \prime 2}}}{\tilde{T}^{2}}+\widetilde{\frac{Y_{f}^{\prime \prime} Y_{o}^{\prime \prime}}{\tilde{Y}_{f} \tilde{Y}_{o}}}+\ldots\right]
$$




\section{Background on Turbulent Premixed Combustion}

This expression also involves a number of higher order correlations which must be retained, at least up to 20th order, to maintain accuracy [24] and this is impractical. Furthermore this will introduce a large set of modelling parameters. In another words, trying to find a closure model for the mean reaction rate using this approach is not practical. Hence, alternative approaches have been proposed in the past and have been discussed elaborately in $[17 ; 20 ; 22 ; 52]$. It is not possible to review all of these approaches here. Furthermore, the interest of this work is on turbulent premixed combustion and thus the related combustion modelling are reviewed in this section.

The mean reaction rate models involve statistical relationships among fluctuating quantities in turbulent flames. These relationships, or the models, depend on the structure of the small scales lost due to averaging. Furthermore, the models depend on the relativity between the scales of turbulence and that of the flame. Damköhler [69] identified two limiting scenarios; flamelet and non-flamelet combustion.

In flamelet combustion, the flame scales are much smaller than the turbulence scales and vice-verse for non-flamelet combustion. These concepts are explained better using a combustion regime diagram.

The turbulent scales range from the Kolmogorov length scale, $\eta_{k}$, with a characteristic velocity $u_{k}^{\prime}$ to the integral length scale, $\Lambda$, with characteristic velocity $u^{\prime}$. Thus, the integral time scale, $\tau_{t}$, and the Kolmogorov time scale, $\tau_{k}$, are defined respectively as

$$
\tau_{t}=\frac{\Lambda}{u^{\prime}}, \quad \tau_{k}=\frac{\eta_{k}}{u_{k}^{\prime}}
$$




\section{Background on Turbulent Premixed Combustion}

The combustion scales are typically represented by flame front thickness, $\delta$, and speed, $S_{L}^{o}$. Thus, the chemical time scale, $\tau_{c}$, is defined as

$$
\tau_{c}=\frac{\delta}{S_{L}^{o}}
$$

The ratio of the integral turbulence time scales to the chemical time scale is defined as the Damköler number

$$
D a=\frac{\tau_{t}}{\tau_{c}}=\frac{\Lambda / \delta}{u^{\prime} / S_{L}^{o}}
$$

The Karlovitz number is defined as the ratio of the chemical time scale to the small-scale turbulence time scale

$$
K a=\frac{\tau_{c}}{\tau_{k}}=\frac{\delta^{2}}{\eta_{k}^{2}}
$$

The turbulence Reynolds number can also be written [70] as

$$
R e=\frac{u^{\prime} / S_{L}^{o}}{\delta / \Lambda}
$$

if $\nu=\delta S_{L}^{o}$. The relationship among these three non-dimensional numbers are commonly represented using the combustion regime diagram [16;70; 71], which is used to classify the turbulent premixed combustion. Such a diagram is shown in Fig. 2.3.

If the thickness of the flame front is smaller than the integral eddies then chemical time scale, $\tau_{c}$, is faster compared to the integral turbulence time scale, $\tau_{t}$, in high $R e$ turbulence. In this situation, turbulence cannot enter the flame 


\section{Background on Turbulent Premixed Combustion}

front and its structure remains close to laminar flame. This flame front is simply wrinkled by turbulence. This situation is denoted on the regime diagram by $D a>1$ and is known as flamelet combustion regime. When the thickness of the flame front is larger than the size of integral eddies then turbulence time scale, $\tau_{t}$, is faster compared to chemical time scale, $\tau_{c}$, disturbing its internal structure if the turbulence level is large. The turbulent eddies can penetrate the flame front and continuously mixing reactants with products. This regime is denoted on the regime diagram by $D a<1$ and is known as perfectly stirred reactor regime.

Karlovitz number is used to further subdivide the flamelets regime depending on the role played by the small-scale eddies. When the flame scales are much smaller than the turbulence small-scales then the internal structure of the flamelets are not disturbed by the small scales of turbulence also. This situation is simply denoted by $K a<1$ in the regime diagram and $K a=1$ is called the Klimov-Williams line signifying the $\delta=\eta_{k}$ situation. When $K a>1$ but $K a<100$, the reaction zones of the flamelets are intact but the small eddies disturb the preheat zone structure which enhances the heat and mass transport in that zone. This situation is known as thin reaction zones combustion [70] as marked in Fig. 2.3. Most of the practical combustion is expected to span between corrugated and thin reaction zones regime. The review by Driscoll [13] suggests that the evidence for non-flamelet behaviour is sparse. With these understandings, a brief review of the existing modelling for premixed turbulent combustion is presented in the following subsections. 


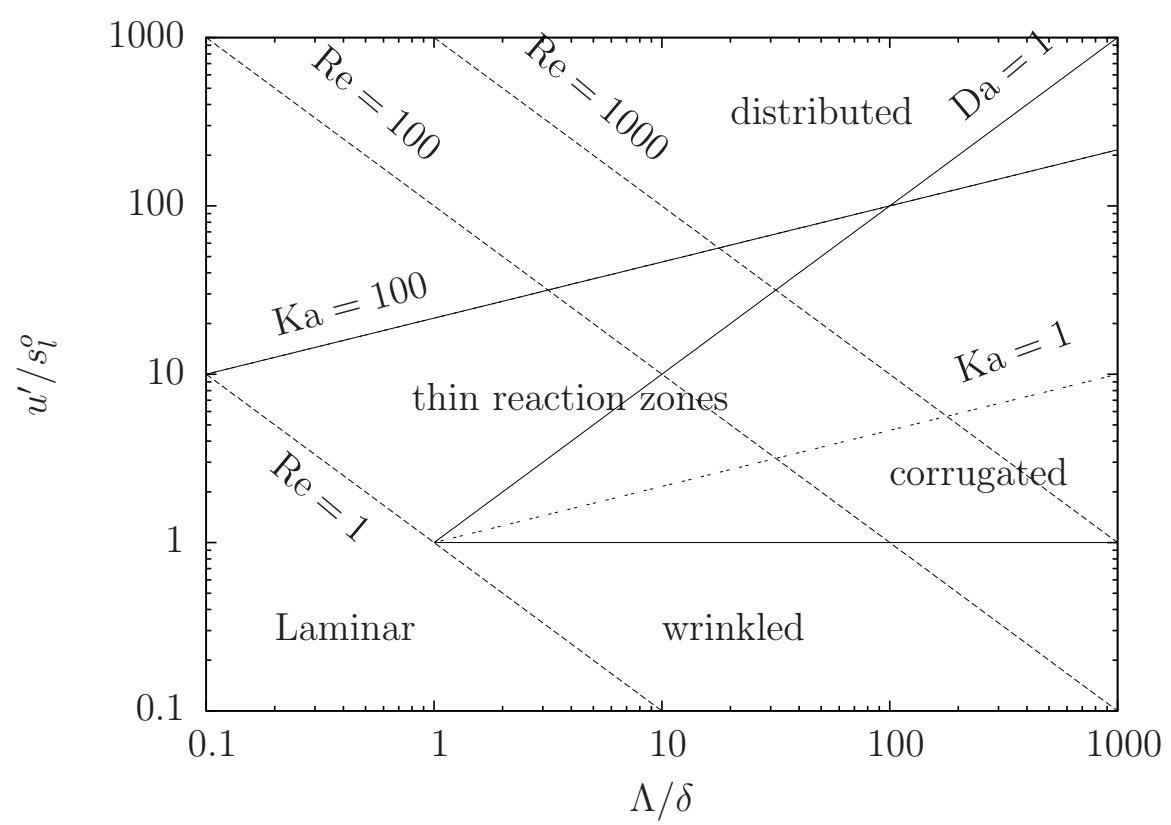

Figure 2.3: The premixed combustion regime diagram.

\subsubsection{Eddy Break-Up Model}

As proposed by Spalding [72] the Eddy Break Up (EBU) model is used for flames with high $R e$ and $D a$ numbers. The basic idea of the model that the reaction zone is described as pockets of unburnt and burnt gases and the turbulent eddies will mix these pockets. Correspondingly, the reaction rate is mainly controlled by this turbulent mixing rate and it is given by

$$
\overline{\dot{\omega}}=C_{E B U} \bar{\rho} \frac{\sqrt{\widetilde{c^{\prime \prime 2}}}}{\tau_{t}}
$$

where, $C_{E B U}$ is the model constant and $\tau_{t}$ is the turbulent time scale which is equal to $\widetilde{k} / \widetilde{\varepsilon}$. An estimation for $\widetilde{c^{\prime \prime 2}}$ based on the assumption of infinitely thin flame yields $\widetilde{c^{\prime \prime 2}}=\widetilde{c}(1-\widetilde{c})$. The EBU model is typically limited to one-step chemistry. 


\subsubsection{Bray-Moss-Libby Model}

Bray-Moss-Libby model (BML) is based on a statistical approach using the probability density function (PDF) of the progress variable, $c$, which is defined to be zero in the reactant and 1 in the product $[73 ; 74 ; 75]$. In order to simplify the analysis the combustion is taken to occur at constant pressure with unity Lewis numbers under adiabatic conditions and the mixture is made of ideal gases. The turbulent premixed flame is taken to be a collection of thin flamelets separating reactants from products implying that at a specific location inside the flame brush and time, reactants are detected for a while and then products are detected for the rest of the time [70;71]. This view allows us to express the PDF of $c$ at a given location to be two delta functions, one at $c=0$ and another at $c=1$ with respective weights of $\alpha$ and $\beta$, and a contribution from the burning parts. Thus,

$$
p(c=\zeta ; \mathbf{x}, t)=\alpha(\mathbf{x}, t) \delta(\zeta)+\beta(\mathbf{x}, t) \delta(1-\zeta)+\gamma(\mathbf{x}, t) f(\zeta),
$$

where, $\zeta$ is the sample space variable for $c$ and $\alpha+\beta+\gamma=1$. For high $R e$ and $D a$ numbers, the flame front is thin and the probability of finding burning gases is very low compared to finding unburnt or burnt mixture therefore, the last term in Eq. (2.46) is neglected. The coefficients $\alpha$ and $\beta$ can be determined from the Favre mean progress variable which is obtained from its transport equation, Eq. (2.28) as follows:

$$
\bar{\rho} \widetilde{c}=\int_{0}^{1} \rho(\zeta) \zeta p(\zeta) d \zeta=\rho_{b} \beta
$$




\section{Background on Turbulent Premixed Combustion}

after using Eq. (2.46) with $\gamma \simeq 0$. The coefficients $\alpha$ and $\beta$ can now be written as $[74]$

$$
\alpha=\frac{1-\widetilde{c}}{1+\tau \widetilde{c}} ; \quad \beta=\frac{(1+\tau) \widetilde{c}}{1+\tau \widetilde{c}}
$$

where $\tau=\left(\rho_{u} / \rho_{b}\right)$ - 1. Now, the mean density is given [58] by $\bar{\rho}=\rho_{u} /(1+\tau \widetilde{c})$ and $\bar{c}=(1-\tau) \tilde{c} /(1+\tau \tilde{c})$. Since the burning mode part of the PDF is neglected $(\gamma=0)$ in the BML approach, an alternative methodology is required to close the mean reaction rate. Bray $[75 ; 76]$ showed that

$$
\overline{\dot{\omega}}_{c}=2 \frac{\bar{\rho} \widetilde{\varepsilon}_{c}}{2 c_{m}-1}
$$

in the limit of $\gamma \rightarrow 0$ by noting the relationship between $\tilde{c}$ and $\widetilde{c^{\prime \prime 2}}$. The model constant $c_{m}$ is given by

$$
c_{m}=\frac{\overline{c \dot{\omega}_{c}}}{\overline{\dot{\omega}}_{c}}=\frac{\int_{0}^{1} \zeta \dot{\omega}_{c} p(\zeta) d(\zeta)}{\int_{0}^{1} \dot{\omega}_{c} p(\zeta) d(\zeta)},
$$

and its typical values vary from 0.7 to 0.8 . The mean scalar dissipation rate, $\tilde{\epsilon}_{c}$, in Eq. (2.49) is defined as

$$
\rho \tilde{\epsilon_{c}}=\overline{\rho D \frac{\partial c^{\prime \prime}}{\partial x_{i}} \frac{\partial c^{\prime \prime}}{\partial x_{i}}}
$$

Models such as $\tilde{\epsilon_{c}}=\widetilde{c^{\prime \prime 2}} / \tau_{t}$ have been used in the past studies, where $\tau_{t}=\tilde{k} / \tilde{\varepsilon}$. Recent developments of this approach for non-negligible $\gamma$ have been discussed elaborately by Bray in reference [16].

Another approach to close the mean reaction rate is based on the flame cross- 


\section{Background on Turbulent Premixed Combustion}

ing frequency analysis. Bray and Libby [77] observed that the mean reaction rate at a given location depends on the frequency at which the flame front crosses this location in turbulent flow. In this analysis, the mean reaction rate is expressed as the product of the flame crossing frequency, $f_{c}$, and the reaction rate per flame crossing, $\dot{\omega}_{f},[77]$ as .

$$
\overline{\dot{\omega}}_{c}=\omega_{f} f_{c}
$$

The flame crossing frequency, $f_{c}$, can be estimated as

$$
f_{c}=2 \frac{\bar{c}(1-\bar{c})}{\tau_{c}}
$$

where $\tau_{c}$ is the mean period of a telegraphic signal, which is used to represent the instantaneous c. This period can be estimated from a turbulence time scale $\tau_{t}=\widetilde{k} / \widetilde{\varepsilon}$. The reaction rate per flame crossing, $\dot{\omega}_{f}$, is modelled as

$$
\dot{\omega}_{f}=\frac{\rho_{u} S_{L}^{0}}{\delta_{L}^{0} / t_{t}}
$$

where $t_{t}$ is the transit time and is defined as the time required to cross the flame front [77].

Bradley [78] suggested that

$$
\overline{\dot{\omega}}_{c}=\iint \dot{\omega}(c, a) p(c, a) d c d a
$$

by assuming that the local flamelets are stretched by turbulent eddies. The stretching effects include the influences of straining and bending of the flame front by turbulent eddies. The symbol $a$ denotes the turbulent stretch and $\dot{\omega}(c, a)$ rep- 
resents the reaction rate in stretched flamelets. Recently, an equivalent approach using the scalar dissipation rate, instead of the stretch rate, has been developed $[79 ; 80]$.

\subsubsection{Flame Surface Density Model}

The flame surface density (FSD) model was originally proposed [81] for nonpremixed flames and was developed later [82; 83] for premixed flames. This approach assumes that the flame propagates locally as a laminar flame and thus the mean reaction rate is expressed as

$$
\overline{\dot{\omega}}_{c}=\rho_{u} S_{L} \Sigma
$$

where $\Sigma$ is the flame surface area per unit volume and $S_{L}$ is the local laminar flame speed accounting for the turbulent stretch. This flame speed is typically expressed as $S_{L}=I_{0} S_{L}^{0}$, where $I_{0}$ is known as the stretch factor [84] and its typical values range from 0.9 to 1 .

One can estimate the flame surface density $\Sigma$ either from algebraic expressions or by solving exact balance equation for $\Sigma$ with suitable closure. A balance equation for $\Sigma$ was first proposed by [81] for non-premixed flames followed by more concrete mathematical derivation based on geometrical $[82 ; 85]$ and statistical analysis [83; 86]. Pope [83] showed that $\Sigma$ associated with a specific iso-surface of progress variable, $c=c *$, as

$$
\Sigma\left(c^{*} ; \underline{\mathrm{x}}, t\right)=\left\langle\overline{|\nabla c|} \mid c=c^{*}\right\rangle p\left(c^{*} ; \underline{\mathrm{x}}, t\right)
$$




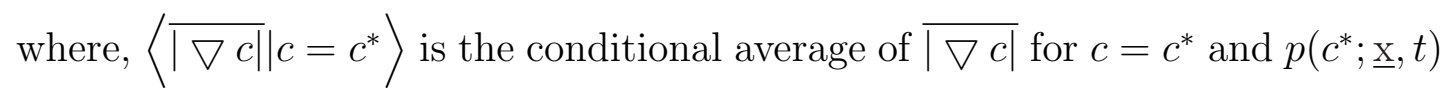
is the PDF.

A balance equation for $\Sigma$ can be written $[82 ; 83 ; 85]$ as

$$
\frac{\partial \Sigma}{\partial t}+\frac{\partial}{\partial x_{i}}\left(\left\langle u_{i}\right\rangle_{s} \Sigma\right)+\frac{\partial}{\partial x_{i}}\left(\left\langle s_{d} n_{i}\right\rangle_{s} \Sigma\right)=\left\langle\left(\sigma_{i j}-n_{i} n_{j}\right) \frac{\partial u_{i}}{\partial x_{i}}\right\rangle_{s} \Sigma+\left\langle s_{d} \frac{\partial n_{i}}{\partial x_{i}}\right\rangle_{s} \Sigma,
$$

where $s_{d}$ is the flame surface displacement speed and is defined as

$$
s_{d}=\left(\frac{1}{|\nabla c|} \frac{D c}{D_{t}}\right)_{c=c^{*}}
$$

$\left(\sigma_{i j}-n_{i} n_{j}\right)$ is the tangential strain rate and $n_{i}$ is the $i t h$ component of the unit vector normal to the flame surface. The modelling of the various terms in Eq. (2.58) has been the subject of many past studies and the important results have been summarised in the books $[16 ; 20 ; 22 ; 84]$ and review paper [17].

\subsubsection{The Probability Density Function}

The Probability Density Function based approach is a statistical description of the problem. For a given location, $x$, and time, $t$, in the flame, the PDF measures the probability to find a variable $\psi$ within $[\psi-\triangle \psi / 2, \psi+\triangle \psi / 2]$ and satisfies the condition.

$$
\int_{\psi_{1}, \psi_{2}, . . \psi_{N}} p\left(\psi_{1}, \psi_{2}, . . \psi_{N}\right) d\left(\psi_{1}, \psi_{2}, . . \psi_{N}\right)=1
$$




\section{Background on Turbulent Premixed Combustion}

where $\psi_{1}, \psi_{2}, . . \psi_{N}$ are the sample space variables for the density, temperature, mass fraction, velocity, etc. The mean reaction rate can be written as

$$
\overline{\dot{\omega}}_{c}=\int_{\psi_{1}, \psi_{2}, . . \psi_{N}} \dot{\omega}\left(\psi_{1}, \psi_{2}, . . \psi_{N}\right) p\left(\psi_{1}, \psi_{2}, . . \psi_{N}\right) d\left(\psi_{1}, \psi_{2}, . . \psi_{N}\right)
$$

where $\dot{\omega}\left(\psi_{1}, \psi_{2}, . . \psi_{N}\right)$ is the functional form of the instantaneous reaction rate. The mean value of, say, $\psi_{1}$ can be simply obtained as

$$
\bar{\psi}_{1}=\int_{\psi_{1}, \psi_{2}, . . \psi_{N}} \psi_{1} p\left(\psi_{1}, \psi_{2}, . . \psi_{N}\right) d\left(\psi_{1}, \psi_{2}, . . \psi_{N}\right)
$$

The challenge now is how to determine the joint PDF, $p$. Two different approaches are commonly used.

The first approach is to solve a transport equation for the joint probability density function $[29 ; 87]$. The advantage of this approach is that the chemical reaction does not require any modelling. However, one major drawback is the computational expense in solving the PDF transport equation, which is written $[29 ; 87]$ as

$$
\begin{gathered}
\frac{\partial \bar{\rho} \tilde{p}(\underline{\psi})}{\partial t}+\frac{\partial}{\partial x_{i}}\left[\bar{\rho} \tilde{u}_{i} \tilde{p}(\underline{\psi})\right]+\sum_{\alpha=1}^{n} \frac{\partial}{\partial \psi_{\alpha}}\left[\frac{1}{\rho} \dot{\omega}_{\alpha}(\underline{\psi}) \bar{\rho} \tilde{p}(\underline{\psi})\right]= \\
-\frac{\partial}{\partial x_{i}}\left[\left\langle u_{i}^{\prime \prime} \mid \underline{\psi}\right\rangle \bar{\rho} \tilde{p}(\underline{\psi})\right]+\sum_{\alpha=1}^{n} \frac{\partial}{\partial \psi_{\alpha}}\left[\left\langle\frac{1}{\rho} \frac{\partial J_{i, \alpha}}{\partial x_{i}} \mid \underline{\psi}\right\rangle \bar{\rho} \tilde{p}(\underline{\psi})\right] .
\end{gathered}
$$

The first, second and third terms on the LHS of Eq. (2.63) are respectively the rate of change of $\tilde{p}(\underline{\psi})$, convective transport and the flux of the PDF in the sample space due to chemical reaction. The first and the second terms on the RHS represent turbulent convection and molecular diffusion effects respectively. 


\section{Background on Turbulent Premixed Combustion}

The vector $\psi$ represents $\left(Y_{1}, Y_{2}, \ldots Y_{N}, T\right)$. The terms on the LHS are closed. The turbulent flux is usually closed using gradient assumption. The molecular diffusion term requires a closure, several mixing models are proposed in past studies. The details of this approach has been reviewed recently by Haworth [88] and Haworth \& Pope [89]. The application of this approach for turbulent premixed flames has been discussed in detail by Lindstedt [90].

In the second approach, a shape for the PDF is presumed and it is usually for the marginal PDF of the progress variable. A presumed shape for the joint PDF has also been attempted in the past [91] but involves a number of uncertainties. The BML PDF in section 2.5.2 is a good example for the presumed PDF approach. It is also typical to use a $\beta$-function [16] for given values of mean and variance. The Favre $\beta$-PDF is given by

$$
\widetilde{p}(\zeta)=\frac{\zeta^{a-1}(1-\zeta)^{b-1}}{\beta(a, b)}
$$

where,

$$
a=\tilde{c}\left(\frac{1-g}{g}\right), b=(1-\tilde{c})\left(\frac{1-g}{g}\right)
$$

where $g=\widetilde{c^{\prime \prime 2}} / \tilde{c}(1-\tilde{c})$ is the variance parameter and

$$
\beta(a, b)=\int_{0}^{1} \zeta^{a-1}(1-\zeta)^{b-1} d \zeta
$$

The values of $\tilde{c}$ and $\widetilde{c^{\prime \prime 2}}$ are usually obtained by solving their respective transport equations. The Favre PDF, $\tilde{p}$, is related to the Reynolds PDF through $p \rho=\bar{\rho} \tilde{p}$ [16]. 


\subsubsection{G-Equation Model}

The G-equation model is based on the flamelet modelling assumption, whereby the chemical reaction occurs within the flame front, which separates the unburned and burned mixtures and moves with a burning velocity normal to the front $S_{T}$.

In this approach, the flame propagation is described as the propagation of an iso-surface of $G(x, t)$. A transport equation for $G$ is derived [24] to track the flame propagation by considering an arbitrary iso-scalar value $G_{0}$ with $G<G_{0}$ representing the unburnt mixture and $G>G_{0}$ representing the burnt mixture. This method has been developed and discussed elaborately by Peters [70]. Basically, a transport equation for the mean $G$-field, written as,

$$
\frac{\partial \bar{\rho} \widetilde{G}}{\partial t}+\frac{\partial \bar{\rho} \widetilde{u}_{i} \widetilde{G}}{\partial x_{i}}=\bar{\rho} S_{T}\left|\frac{\partial \widetilde{G}}{\partial x_{i}}\right|-\bar{\rho} D_{t} \kappa\left|\frac{\partial \widetilde{G}}{\partial x_{i}}\right|,
$$

is solved with models for $S_{T}, D_{t}$ and $\kappa$ along with a transport equation for $\widetilde{G^{\prime \prime 2}}$. The detail of this method is discussed by Peters [70] and Peters and Bray [92]. This formulation is valid only for the corrugated flamelets regime of combustion [93].

\subsection{Summary}

All the methods described above are used in modelling turbulent premixed combustion but choosing the right method is essential. For example, in the EBU model the reaction rate is controlled by mixing and finite rate chemistry effects are not typically included in the model. The laminar flamelet models are based on the assumption that the flame scales are smaller than the turbulence scales. 
This assumption becomes invalid if one considers some minor species and pollutants. The transported PDF models depend on many variables that make the method computationally expensive.

Conditional moment closure $[27 ; 40]$ is an alternative approach showing a good potential to predict minor and pollutant species of engineering interest with moderate computational cost as one shall note from this study. This method has been used widely for non-premixed flames and it is development and application to premixed flames is sparse. Hence, this method is chosen for this study and is described in detail in the next chapter. 


\section{Chapter 3}

\section{Conditional Moment Closure}

This chapter presents a review of the main features of the conditional moment closure (CMC). The first section begins by presenting the concept and fundamentals of this method. The second and third sections focus on CMC for premixed combustion followed by premixed CMC sub-models.

\subsection{CMC Methodology}

As shown in section 2.5, an accurate closure for the mean reaction rate cannot be found using the conventional moment method. This is because, the fluctuations of various scalar concentrations and temperature over the mean are very large. This large fluctuation along with the strong non-linearity in the reaction rate mainly renders the moment method to be of less use to obtain a closure for the mean reaction rate. However, as Bilger [27] pointed out, the philosophy of the moment method can be adopted if one uses conditional moments rather than the unconditional moments. This is because the fluctuations over the conditional 
mean are small compared to the unconditional fluctuation level. This point is depicted in Fig. 3.1, showing the scatter plot of temperature and $\mathrm{OH}$ mass fraction in mixture fraction space. The corresponding conditional averages are also shown on the left. The solid lines represent the solution of fully burning strained laminar diffusion flame in counter-flow configuration with a strain rate parameter of $5 s^{-1}$. It is seen in the scatter plot that the fluctuations in $T, Y_{O H}$ and $Z$ are large. If one constructs conditional averages, to be defined below, then the fluctuations levels become small as shown on the right hand side of Fig. 3.1. Here the main hypothesis of CMC is that the fluctuations of temperature and scalar mass fraction values are closely associated to the fluctuation in one or two key scalar quantities. Thus, if one constructs averages conditional upon a particular value for the key scalars then there is a hope to find an accurate closure for the mean reaction rate using the concept of moment methods. The mixture fraction and progress variable are good conditioning variables for non-premixed and premixed flames respectively [40].

In $\mathrm{CMC}$, transport equations for the conditional averages are derived and solved along with other moment equations subject to appropriate initial and boundary conditions. First, the instantaneous value of, say mass fraction of scalar $\alpha$, is decomposed into its conditional mean, $Q_{\alpha}$, and fluctuation, $y_{\alpha}^{\prime \prime}$, over $Q_{\alpha}$ as

$$
Y_{\alpha}(\underline{\mathrm{x}}, t)=\left\langle Y_{\alpha} \mid Z=\eta\right\rangle+y_{\alpha}^{\prime \prime}(\underline{\mathrm{x}}, t)=Q_{\alpha}(\eta ; \underline{\mathrm{x}}, t)+y_{\alpha}^{\prime \prime}(\underline{\mathrm{x}}, t)
$$

where the angled brackets denote ensemble averaging subject to the condition on the right of the vertical bar and this average is defined as conditional average. Substituting this decomposition into Eq. (2.4) and then taking the conditional 
average of the resulting equation gives a transport equation for $Q_{\alpha}$. The detail of this derivation is presented clearly by Bilger [27] and Klimenko and Bilger [40]. An appropriate joint PDF transport equation, see for example Eq. (2.63), can also be used to obtain the transport equation for $Q_{\alpha}$ as shown by Klimenko [28]. The similarities and differences between the decomposition and joint PDF methods to obtain $Q_{\alpha}$ transport equation are discussed in detail by Klimenko and Bilger [40] and interested readers are referred to that study. However, both of these methods led to almost the same transport equation for $Q_{\alpha}$, which will be presented in the next section. As one shall see in that section, this transport equation involves some terms, primarily the conditional averages of velocities, reaction rates, and scalar dissipation rate of the key conditioning scalar, requiring closure models. Many models have been developed in past studies and the CMC have been successfully applied to various non-premixed combustion systems such as bagasse-fired boiler [32], hood fires [30], bluff-body stabilised[33; 34] and lifted jet flames [31], spray autoignition [35] and soot formation [36]. The application of CMC to premixed flames is not fully tested and validated yet. Despite some initial results reported in $[94 ; 95 ; 96]$, the main difficulty in applying $\mathrm{CMC}$ to turbulent premixed flames is associated with modelling of conditional scalar dissipation rate, $\left\langle N_{c} \mid c=\zeta\right\rangle=\left\langle D_{c}(\nabla c \cdot \nabla c) \mid \zeta\right\rangle$, of the key conditioning scalar, the progress variable $c$.

Many studies $[16 ; 79 ; 97 ; 98 ; 99]$ have attempted to shed more light on this dissipation rate and its modelling, which encourages to explore CMC for premixed flames. 

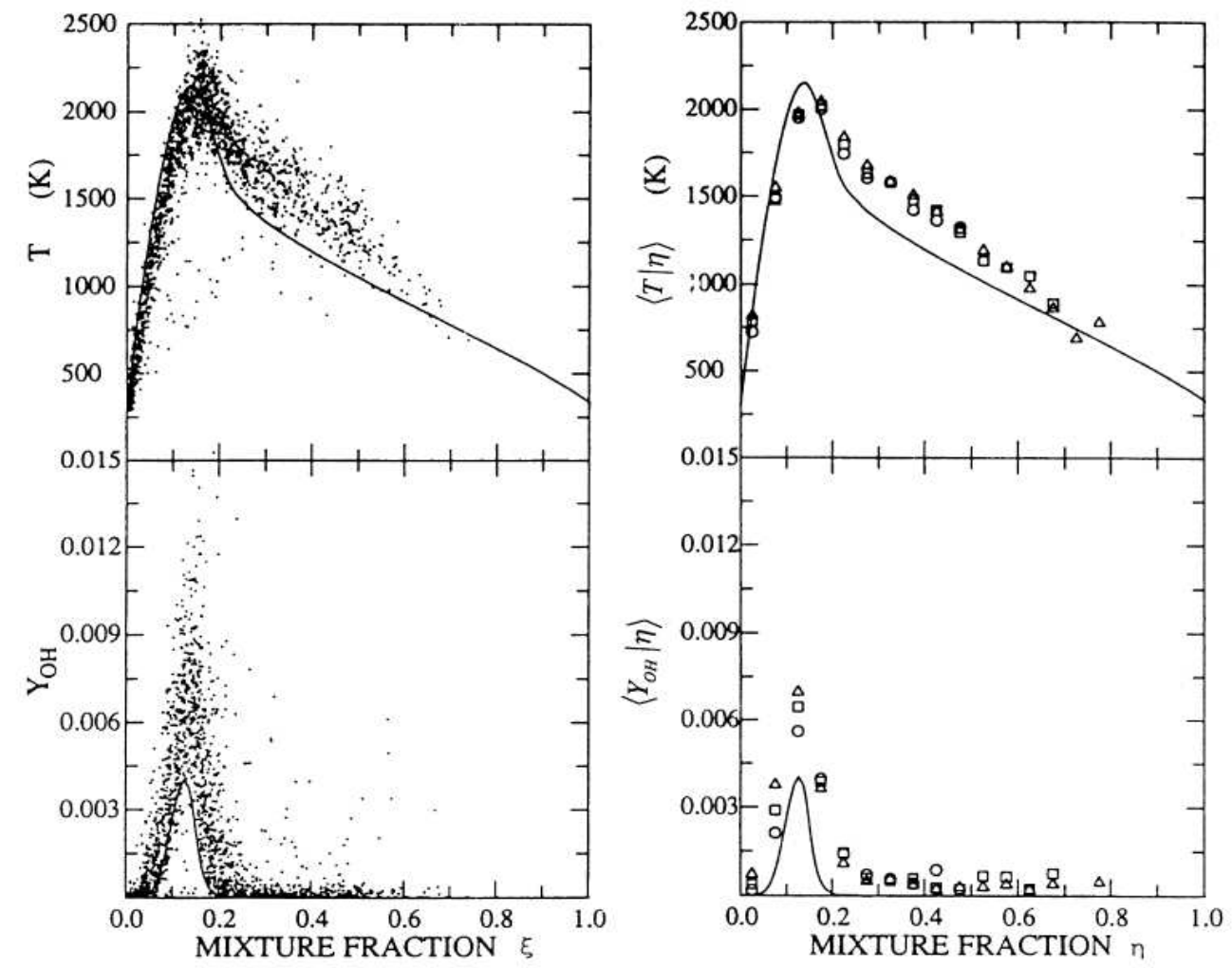

Figure 3.1: Scatter plots of instantaneous and conditional averages for the temperature and $\mathrm{OH}$ mass fraction at $x / D=20$ and $r / D=1.7(\circ) ; 1.4(\square) ; 1.1(\triangle)$ [1]. Solid line represents fully burning strained laminar flame result. 


\subsection{Premixed CMC Method}

As noted earlier, the CMC method is based on the hypothesis that the fluctuations of species mass fractions, temperature and enthalpy are associated only with the fluctuation of a key scalar [40], which is the progress variable, $c$, for premixed combustion. The progress variable is a reactive scalar and it can be defined based on temperature, Eq. (2.11), or using the fuel mass fraction, Eq. (2.12) [17]. An alternative definition using sensible enthalpy is also possible [40]. Here, it is defined as $\theta=Y_{f} / Y_{f}^{u}$ using the fuel mass fraction, where $Y_{f}^{u}$ is the unburnt fuel mass fraction value. This is a progress variable and is used as conditioning variable. The value of $\theta=1$ implies that the mixture is unburnt and $\theta=0$ implies that the mixture is completely burnt.

Transport equations for conditional mean scalar values, $Q_{\alpha}$, are derived by substituting, as noted earlier in Eq. (3.1)

$$
Y_{\alpha}(x, t)=Q_{\alpha}(\zeta ; \mathbf{x}, t)+y_{\alpha}^{\prime \prime}(\mathbf{x}, t)
$$

in the transport equation for the instantaneous scalar value $Y_{\alpha}$, Eq. (2.4) [27]. It is to be noted that density weighted conditional averaging [40] must be used, which is given by $Q_{\alpha}(\zeta ; \mathbf{x}, t)=\left\langle\rho Y_{\alpha} \mid \zeta\right\rangle /\langle\rho \mid \zeta\rangle$, where $\zeta$ is the sample space variable for $\theta$. A full derivation of the $\mathrm{CMC}$ transport equation is given in the Appendix A. It is to be noted that the decomposition method is used because of its simplicity, although one can follow the joint PDF approach of Klimenko [28]. Both of these approaches essentially yield the same transport equation for $Q_{\alpha}$ which is written as $[40 ; 96]$ 


$$
\begin{array}{r}
\langle\rho \mid \zeta\rangle \frac{\partial Q_{\alpha}}{\partial t}+\left\langle\rho u_{i} \mid \zeta\right\rangle \frac{\partial Q_{\alpha}}{\partial x_{i}}-\frac{\operatorname{Le}_{\theta}}{\operatorname{Le}_{\alpha}}\left\langle\rho N_{\theta} \mid \zeta\right\rangle \frac{\partial^{2} Q_{\alpha}}{\partial \zeta^{2}}=\left\langle\dot{\omega}_{\alpha} \mid \zeta\right\rangle-\left\langle\dot{\omega}_{\theta} \mid \zeta\right\rangle \frac{\partial Q_{\alpha}}{\partial \zeta} \\
-\frac{1}{\widetilde{p}} \frac{\partial}{\partial x_{i}}\left[\left\langle\rho u_{i}^{\prime \prime} y_{\alpha}^{\prime \prime} \mid \zeta\right\rangle\langle\rho \mid \zeta\rangle \widetilde{p}\right]+e_{Q_{\alpha}}
\end{array}
$$

where $\mathrm{Le}_{\alpha}$ is the Lewis number of species $\alpha$ and $\widetilde{p}$ is the Favre PDF of $c$. The physical meaning of various terms in Eq. (3.3) is as follows. The first and second terms of Eq. (3.3) respectively denote the unsteady and convective changes of $Q_{\alpha}$. The third term represents the diffusion of the conditional average in the sample space $\zeta$, which is modulated by the non-unity Lewis number effects. The fourth term is the conditional chemical reaction rate for species $\alpha$. Since $\theta$ is a reactive scalar, its influence on the evolution of $Q_{\alpha}$ is given by the fifth term in Eq. (3.3). The sixth term represents the contribution of conditional fluctuation $y_{\alpha}^{\prime \prime}$ to $Q_{\alpha}$ evolution, which comes from the convective term of $e_{y_{\alpha}}$ given in Eq. 16 of Appendix-A. The last term represents contributions of molecular diffusion in the physical space and differential diffusion effects. The explicit from of $e_{Q_{\alpha}}$ is given in Eq. 17 of the Appendix-A.

The CMC transport equations are to be solved along with other governing and modeling equations discussed in sections 2.3 for the RANS methodology. One must also not forget that appropriate initial and boundary conditions are required for the CMC transport equation and these conditions will be discussed while presenting the numerical methods in chapter 5 . In the next section closures for the unclosed terms in Eq. (3.3) are discussed. 


\subsection{Premixed CMC Sub-Models}

The quantities $\left\langle u_{i}^{\prime \prime} y_{\alpha}^{\prime \prime} \mid \zeta\right\rangle, \widetilde{p},\left\langle u_{i} \mid \zeta\right\rangle,\left\langle\dot{\omega}_{\alpha} \mid \zeta\right\rangle,\left\langle\dot{\omega}_{\theta} \mid \zeta\right\rangle, e_{Q_{\alpha}},\langle\rho \mid \zeta\rangle$ and $\left\langle N_{\theta} \mid \zeta\right\rangle$ require suitable models. The PDF, $\widetilde{p}$, is modelled using a presumed shape with a Beta function, Eq. (2.64).

The conditional turbulent scalar flux, $\left\langle u_{i}^{\prime \prime} y_{\alpha}^{\prime \prime} \mid \zeta\right\rangle$, is generally closed using gradient transport hypothesis for non-premixed flames [40], although it is also possible for this conditional flux to be counter-gradient [100] and new modelling methods are to be developed to account for this. However, the adequacy of the gradient flux model for this study can be judged from the results to be presented in chapter 6 , and it is written as

$$
\left\langle u_{i}^{\prime \prime} y_{\alpha}^{\prime \prime} \mid \eta\right\rangle=-\frac{\mu_{t}}{S c_{\alpha}} \frac{\partial Q_{\alpha}}{\partial x_{i}}
$$

where $S c_{\alpha}$ is the turbulent Schmidt number for species $\alpha$, which is typically taken to be 0.7 . This model is reasonable for high Re flows and it has been used in the past for RANS and LES simulations [32; 80] and [101].

The conditional mean reaction rate, $\left\langle\dot{\omega}_{\alpha} \mid \zeta\right\rangle$, for species $\alpha$ in Eq. (3.3) is closed using a first order CMC closure [40]. According to this closure, the conditional mean reaction rate has the functional dependence on the conditional mean as the instantaneous reaction rate has on the instantaneous scalar values. Hence,

$$
\left\langle\dot{\omega}_{\alpha} \mid \zeta\right\rangle=\dot{\omega}_{\alpha}\left(\langle\rho \mid \zeta\rangle, \mathbf{Q}_{\alpha}, Q_{T}\right)
$$


where $Q_{T}$ is the conditional mean temperature. This first order closure is shown [40] to be accurate for non-premixed flames when there is no local extinction or ignition events present in the flow. A prior analyses [96] using DNS data showed that this closure is also accurate for premixed flames and thus it is used in this study. Since the transport equations for $Q_{\alpha}$ are solved along with appropriate initial and boundary conditions, an arbitrarily complex chemical kinetics can be included in the CMC.

The closure of $\left\langle\dot{\omega}_{\theta} \mid \zeta\right\rangle$ is based on the definition of $\theta$. Since $\theta$ is defined based on the fuel mixture fraction

$$
\left\langle\dot{\omega}_{\theta} \mid \zeta\right\rangle=\frac{\left\langle\dot{\omega}_{f} \mid \zeta\right\rangle}{Y_{C H_{4}}^{u}}
$$

The conditional mean velocity components are closed using a linear model [40], which has been used for non-premixed flames. This model is given by

$$
\left\langle u_{i} \mid \zeta\right\rangle=\tilde{u_{i}}+\frac{\widetilde{u_{i}^{\prime \prime} \theta^{\prime \prime}}}{\widetilde{\theta^{\prime \prime 2}}}(\zeta-\tilde{c})
$$

where, $\tilde{u}_{i}$ is the unconditional Favre mean velocity, $\widetilde{u_{i}^{\prime \prime} \theta^{\prime \prime}}$ is the correlation between the velocity fluctuations and the progress variable fluctuations, $\widetilde{\theta^{\prime \prime 2}}$ is the variance of the progress variable fluctuations. This model showed good agreement with DNS results [96] for turbulent premixed flames. Although other modellings are possible [96], this linear model is used because it is simple and sufficiently accurate. 
From the Eq. 17 of Appendix A,

$$
\begin{array}{r}
e_{Q_{\alpha}} \equiv\left\langle\frac{\partial}{\partial x_{i}}\left(\rho D_{\alpha} \frac{\partial Q_{\alpha}}{\partial x_{i}}\right)+\rho D_{\alpha} \frac{\partial \theta}{\partial x_{i}} \frac{\partial}{\partial x_{i}}\left(\frac{\partial Q_{\alpha}}{\partial \zeta}\right) \mid \zeta\right\rangle+ \\
\left\langle\frac{\partial Q_{\alpha}}{\partial \zeta} \frac{\partial}{\partial x_{i}}\left[\left(1-\frac{\mathrm{Le}_{\alpha}}{\mathrm{Le}_{\theta}}\right) \rho D_{\alpha} \frac{\partial \theta}{\partial x_{i}}\right] \mid \zeta\right\rangle
\end{array}
$$

The molecular diffusion contributions are negligible for high Reynolds number flows and the differential diffusion effect, the third term in Eq. (3.8) remains as the dominant term [96]. Thus,

$$
\begin{aligned}
& e_{Q_{\alpha}}=\left(1-\frac{\mathrm{Le}_{\alpha}}{\mathrm{Le}_{\theta}}\right) \frac{\partial Q_{\alpha}}{\partial \zeta}\left[\rho D_{\alpha} \frac{\partial \tilde{\theta}}{\partial x_{i}}\right] \\
& e_{Q_{\alpha}}=\left(1-\frac{\mathrm{Le}_{\alpha}}{\operatorname{Le}_{\theta}}\right) \frac{\partial Q_{\alpha}}{\partial \zeta} \frac{1}{\tilde{p}} \frac{\partial\left\langle N_{\theta} \mid \zeta\right\rangle \tilde{p}}{\partial \zeta} .
\end{aligned}
$$

The second part of Eq. (3.10) is obtained after using the inter-relationship between the conditional diffusion and conditional dissipation in high Re turbulent flames [40;96]. It is apparent that the contribution of $e_{Q_{\alpha}}$ depends on $\mathrm{Le}_{\alpha}$, if $\mathrm{Le}_{\alpha} / \mathrm{Le}_{\theta}=1$, this contribution is zero. It is to be noted that this closure requires a model for the conditional dissipation rate $\left\langle N_{\theta} \mid \zeta\right\rangle$, which is discussed in chapter 4 .

The conditional density, $\langle\rho \mid \zeta\rangle$, is obtained using the state equation and the conditional temperature, $Q_{T}$, through

$$
\langle\rho \mid \zeta\rangle=\frac{P}{R Q_{T}}
$$




\section{Conditional Moment Closure}

where $R$ is the characteristics gas constant.

As noted earlier in section 3.1, the modelling of the conditional and unconditional dissipation rate of the progress variable offer significant challenges, since the progress variable is a reactive scalar. This is discussed in the next chapter. 


\section{Chapter 4}

\section{Scalar Dissipation Rate}

The effect of turbulence on premixed flames is to increase the flame surface area by wrinkling the flame front [24]. In premixed flames, mixing of cold reactants and hot products on the flame surface, is crucial to sustain the combustion. This mixing phenomenon is characterised by the scalar dissipation rate, $N_{\theta}$, which may be broadly defined as the rate of mixing at a molecular level. This term appears in many turbulent combustion models such as flamelets [102], probability density function [29] and conditional moment closure [40] and requires an accurate model. A considerable amount of experimental and modelling literature has been published on the scalar dissipation rate for non-premixed [17; 103; 104; 105; 106] and for premixed $[79 ; 97 ; 98 ; 107 ; 108]$ combustion. Nevertheless, in premixed flames, a sensible model for the scalar dissipation rate must account for the interaction between turbulence, chemical reaction and molecular diffusion and thus using turbulent time scale alone proved to be insufficient [97; 109].

Recent development in the modelling of the mean dissipation rate is explored briefly in section 4.1. The modellings of unconditional dissipation rate are dis- 
cussed in section 4.2. The validation of these models are presented in section 4.3, before choosing a suitable model for the CMC calculation.

\subsection{Mean Scalar Dissipation Rate}

An exact transport equation for the dissipation rate has been derived and analysed to alleviate these issues [97]. This analysis and the insights obtained using this transport equation in a number of studies have been summarised by Chakraborty et al., [110]. For the purpose of this study, the final result, an algebraic model for the mean scalar dissipation rate, $\left.\widetilde{\epsilon_{\theta}}=\overline{\rho \mathcal{D}_{\theta}\left(\nabla \theta^{\prime \prime} \cdot \nabla \theta^{\prime \prime}\right.}\right) / \bar{\rho}$, is employed. This algebraic model [98] is obtained by analysing the closure models proposed in [99] for the leading order terms of the mean scalar dissipation rate transport equation, when the Damköler number is large. This model, validated using DNS and experimental data [99], is written as

$$
\widetilde{\epsilon_{\theta}}=\frac{1}{\beta^{\prime}}\left[\left(2 K_{c}^{*}-\tau C_{4}\right) \frac{S_{L}^{0}}{\delta_{L}^{0}}+C_{3} \frac{\tilde{\varepsilon}}{\tilde{k}}\right] \widetilde{\theta^{\prime \prime 2}}
$$

where, $\beta^{\prime}=6.7, K_{c}^{*}=0.85 \tau$ for hydrocarbon-air flames, $C_{4}=1.1 /(1+K a)^{0.4}$ and $C_{3}=1.5 \sqrt{K a} /(1+\sqrt{K a})$. The Karlovitz number is defined as

$$
K a=\left\{\frac{1}{2(1+\tau)^{0.7}}\left(\frac{u^{\prime}}{S_{L}^{\circ}}\right)^{3}\left(\frac{\delta_{L}^{\circ}}{\Lambda}\right)\right\}^{0.5} .
$$

The Favre averaged turbulence kinetic energy and its dissipation rate are denoted by $\widetilde{k}$ and $\widetilde{\varepsilon}$ respectively. 


\subsection{Conditional Mean Scalar Dissipation Rate}

The conditional mean scalar dissipation rate, $\langle N \mid \theta\rangle$, is related to the unconditional mean dissipation rate through

$$
\tilde{\epsilon_{\theta}}=\int_{0}^{1}\left\langle N_{\theta} \mid \zeta\right\rangle \tilde{p}(\zeta) d \zeta
$$

Thus, a closure for $\left\langle N_{\theta} \mid \zeta\right\rangle$ can be obtained from $\widetilde{\epsilon_{\theta}}$ using two different approaches; one is based on physical understanding and the other one is by directly solving the integral equation in Eq. (4.3). Both of these are explored below, before choosing a model for the CMC calculations.

\subsubsection{Algebraic Model}

It is well known that the turbulent stretch influences the local reaction rates in premixed flames. Thus, the local scalar gradients will also be clearly influenced by the stretch rate since the scalar gradients are produced predominately by chemical reactions in premixed flames. If one considers the local flame front as stretched flamelets then Kolla and Swaminathan [79] showed that the dissipation rate in the stretched flamelets, subject to a stretch rate $a$, can be expressed as $N(\theta, a)=N\left(\theta^{*}, a\right) f(\theta)$, where $\theta$ is the conditioning variable and $\theta^{*}$ is its value at the inner reaction zone. The inner reaction zone is defined as the location where the fuel molecule will be attacked by a hydrogen atom and the typical value of $\theta^{*}$ is about 0.3 for common hydrocarbon fuels.

If one considers the turbulent flame-brush as a collection of stretched laminar 
flames then the mean dissipation rate is given by

$$
\begin{gathered}
\widetilde{\epsilon_{\theta}}=\int_{0}^{1} \int_{0}^{\infty} N(\theta, a) p(\theta, a) d a d \theta \\
\widetilde{\epsilon_{\theta}}=\int_{0}^{1}\langle N \mid \theta\rangle \tilde{p}(\theta) d \theta \\
\widetilde{\epsilon_{\theta}}=\left\langle N \mid \theta^{*}\right\rangle \int_{0}^{1} f(\theta) \tilde{p}(\theta) d \theta .
\end{gathered}
$$

Replacing $\left\langle N \mid \theta^{*}\right\rangle=\langle N \mid \theta\rangle / f(\theta)$ one gets

$$
\langle N \mid \theta\rangle=\frac{\tilde{\epsilon} f(\theta)}{\int_{0}^{1} f\left(\theta^{\prime}\right) \tilde{p}\left(\theta^{\prime}\right) d\left(\theta^{\prime}\right)},
$$

as a model for the conditional dissipation rate [79]. The function $f(\theta)$ obtained from the unstrained laminar premixed flame is shown to be a very good approximation by Kolla and Swaminathan [79]. If one knows $\tilde{\theta}$ and $\widetilde{\theta^{\prime \prime 2}}$ then $\langle N \mid \theta\rangle$ can be obtained by presuming the PDF, $\tilde{p}$, as in Eq. (2.64), and using Eq. (4.1) for $\widetilde{\epsilon_{\theta}}$. The validation of this model will be discussed in section 4.3.

\subsubsection{Inverse Model}

The second method to obtain the conditional scalar dissipation rate is based on finding a solution [111] for the ill-posed integral equation in Eq. (4.3). Ill-posed problems arise in many science and engineering applications, which have a nonunique and/or an unstable solution. To explain this, let's consider a Fredholm integral equation of the first kind as

$$
F(y)=\int_{a}^{b} K(x, y) G(x) d x, \quad c \leq y \leq d
$$


where, $G(x)$ is unknown function, $F(y)$ and the kernel $K(x, y)$ are approximately known functions. It can be written in matrix form as

$$
K G=F,
$$

where $K$ is the $n \times n$ matrix, $F$ is $n \times 1$ matrix and $G$ is $n \times 1$. The solution for Eq. (4.9) would be given by

$$
G \simeq K^{-1} F
$$

and if the problem is well-posed then a unique solution will exist. But these problems are characterised as ill-posed system when the solution $G$ is very sensitive to small changes or errors in $F(y)$ or $K(x, y)$, because the matrix $K$ may have local singularities. For these situations, a regularisation method is adopted usually to find a stable and meaningful solution. Out of several regularisation method available Tikhonov regularisation algorithm [111] is used in this study to find an approximate solution for the conditional scalar dissipation rate from Eq. (4.3).

This regularisation method translates the problem into a minimisation problem written as

$$
\left.\min \left\{\left|\tilde{p} N_{\theta}^{\alpha}-\tilde{\epsilon_{\theta}}\right|^{2}+\alpha\left|\left(N_{\theta}^{\alpha}-N_{\theta}^{\alpha-1}\right)\right|^{2}\right)\right\}
$$

where, $\alpha \geq 0$ is the regularisation parameter. The notation $N_{\theta}^{\alpha}$ implies the value of $N_{\theta}$ obtained with regularisation parameter $\alpha$. The value of this parameter evolves as $\alpha_{j}=e \alpha_{j-1}$ while solving the above minimisation using an iterative technique, where $j$ is the iteration index and $0<e<1$. Thus, the final converged 
solution for $N_{\theta}$ will strongly depend on its initial guess value and the behaviour of the kernel $\tilde{p}$.

\subsection{Model Validation}

\subsubsection{DNS Database}

The above two models are validated using two different sets of DNS database having different thermo-chemical and turbulence conditions. A brief discussion of these databases is presented first before discussing the validation results.

Direct numerical simulations of turbulent premixed V-flames of methane-air mixture of equivalence ratio 0.58 with preheated reactant temperature of $600 \mathrm{~K}$ [112]. The V-flame simulations were carried out using the fully compressible, three-dimensional DNS code SENGA2. The chemical kinetics was simulated [112] using a single irreversible reaction with kinetic parameters giving a value of about $0.6 \mathrm{~m} / \mathrm{s}$ for the unstrained laminar flame speed and about $0.4 \mathrm{~mm}$ for the thermal thickness. The Lewis number was taken to be unity.

The domain is cubic with sides $L_{x}=L_{y}=L_{z}=29.7 \delta_{L}^{\circ}$. Non-reflecting outflows are specified on the downstream and transverse faces, and the remaining direction is periodic. Turbulence is supplied at the inlet from a pre-computed frozen solution of fully developed, homogeneous isotropic turbulence at the appropriate turbulence intensity. Once inside the V-flame domain, the turbulence freely decays as it interacts with the flame and is convected downstream with the mean velocity. The flame is stabilised at the flame holder by imposing product mass fractions over a small cylindrical region at $x=3.48 \delta_{L}^{\circ}$ from the inlet 
boundary; equivalent to a catalytic wire aligned in the periodic direction.

Once initial transients have decayed, the simulations are run for one complete flow-through time, $\tau_{D}=L_{x} / \bar{u}_{i n}$ where $\bar{u}_{i n}$ is the mean inlet velocity, during which data are saved at regular intervals. All mean quantities are then time and space averaged from ensembles of the saved snapshots and over all points in the periodic direction. To capture the spatial development of the flame, two locations downstream from the flame holder are considered: $X 1=16.7 \delta_{L}^{\circ}$ and $X 2=27.9 \delta_{L}^{\circ}$, at which the influence of the flame holder is known to be negligible. The relevant parameters of these two $\mathrm{V}$ flames are given in table 4.1 and the combustion is in the corrugated flamelets and thin reaction zones regime of turbulent combustion as noted in Fig. 4.3.

\begin{tabular}{ccccccc}
\hline \hline Flame & $u^{\prime} / S_{L}^{\circ}$ & $\Lambda / \delta_{L}^{\circ}$ & $R e$ & $D a$ & $K a$ & $t_{a n}^{*}$ \\
\hline R2a & 0.85 & 78.0 & 106.8 & 91.8 & 0.2 & 1.0 \\
R2c & 3.40 & 19.5 & 106.2 & 5.7 & 3.2 & 2.5 \\
VA & 2.0 & 3.62 & 37 & 1.81 & 1.49 & 1.0 \\
VB & 6.0 & 3.43 & 92 & 0.57 & 7.94 & 6.0
\end{tabular}

Table 4.1: Inlet parameters for the selected DNS flames. $t_{a n}^{*}$ is in terms of the initial eddy turnover for R2a \& R2c flames, it is in term of flow through time for V flames.

The second set of DNS data considered includes turbulent premixed flames of H2-air turbulent premixed flames propagating in three-dimensional homogeneous isotropic turbulence developed at Tokyo Institute of Technology [113; 114] are considered. In this DNS, stoichiometric premixed H2-air flames with preheated reactants at $T_{u}=700 \mathrm{~K}$ were simulated using a multiple-step detailed kinetic mechanism involving 27 reactions and 12 reactive species. These two simulations denoted as R2a and R2c were in the wrinkled flamelets and thin reaction zones 


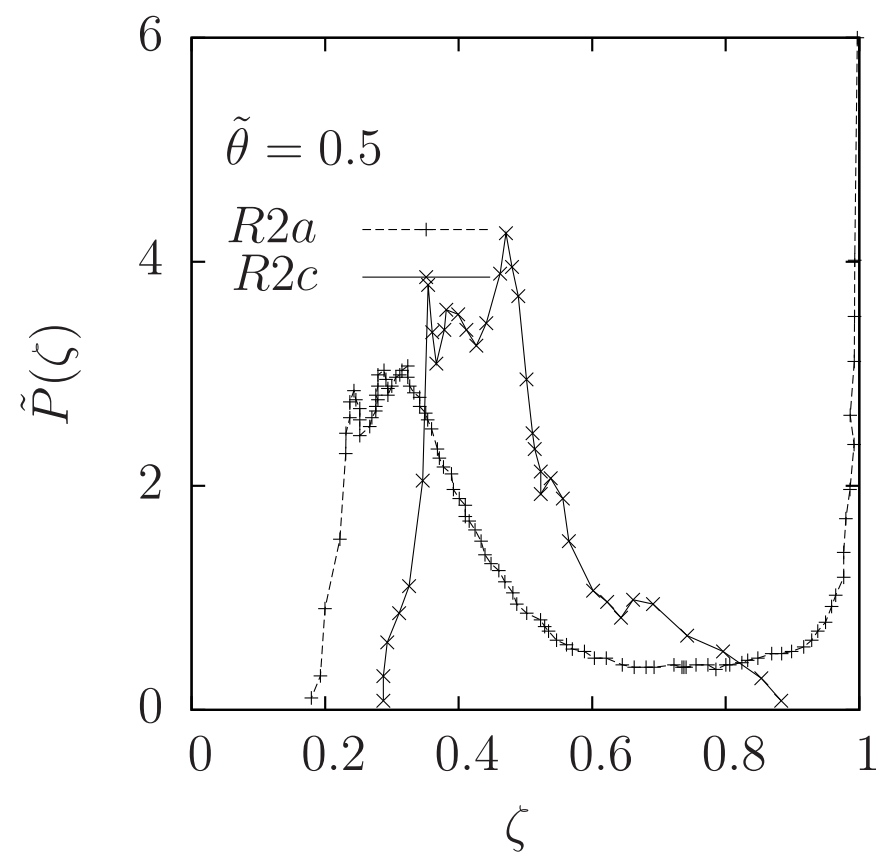

Figure 4.1: The variation of the Favre averaged PDF of the progress variable, $\tilde{p}(\zeta)$, at $\theta=0.5$ inside the flame brush of flames R2a and R2c.

regimes of turbulent combustion as shown in Fig. 4.3. The relevant parameters of these two data sets are listed in table 4.1.

The last column of table 4.1 shows the time of analysis for the H2-air data set and the sampling time for the $\mathrm{V}$ flames. This time is normalised using the initial large eddy turn over time of the turbulence for H2-air flames and the flow through for the $\mathrm{V}$ flames. These data sets are analysed to validate the models for the conditional dissipation rate discussed in the previous section. Note that the results from $\mathrm{V}$ flames are obtained at two different axial locations in the flame; $x_{1}=16.7 \delta_{L}^{\circ}$ and $x_{2}=27.9 \delta_{L}^{\circ}$.

Figs. 4.1 and 4.2 show the Favre PDF of the progress variable $\tilde{\theta}$ for flames R2a and R2c at $\theta=0.5$ and flames VA and VB at $\theta=0.6$. 

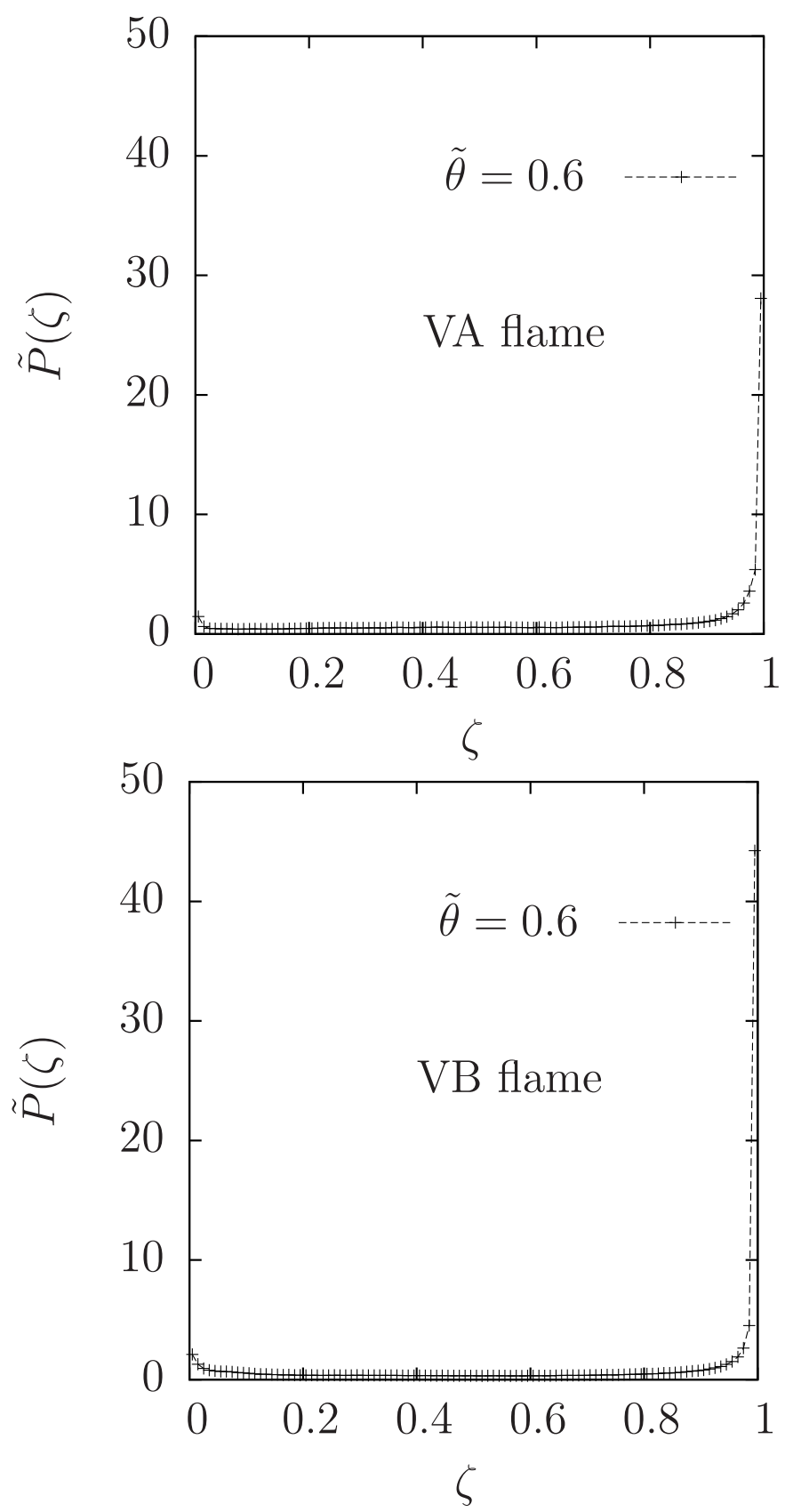

Figure 4.2: The variation of the Favre averaged PDF of the progress variable, $\tilde{p}(\zeta)$, at $\theta=0.6$ inside the flame brush of flames VA and VB. 


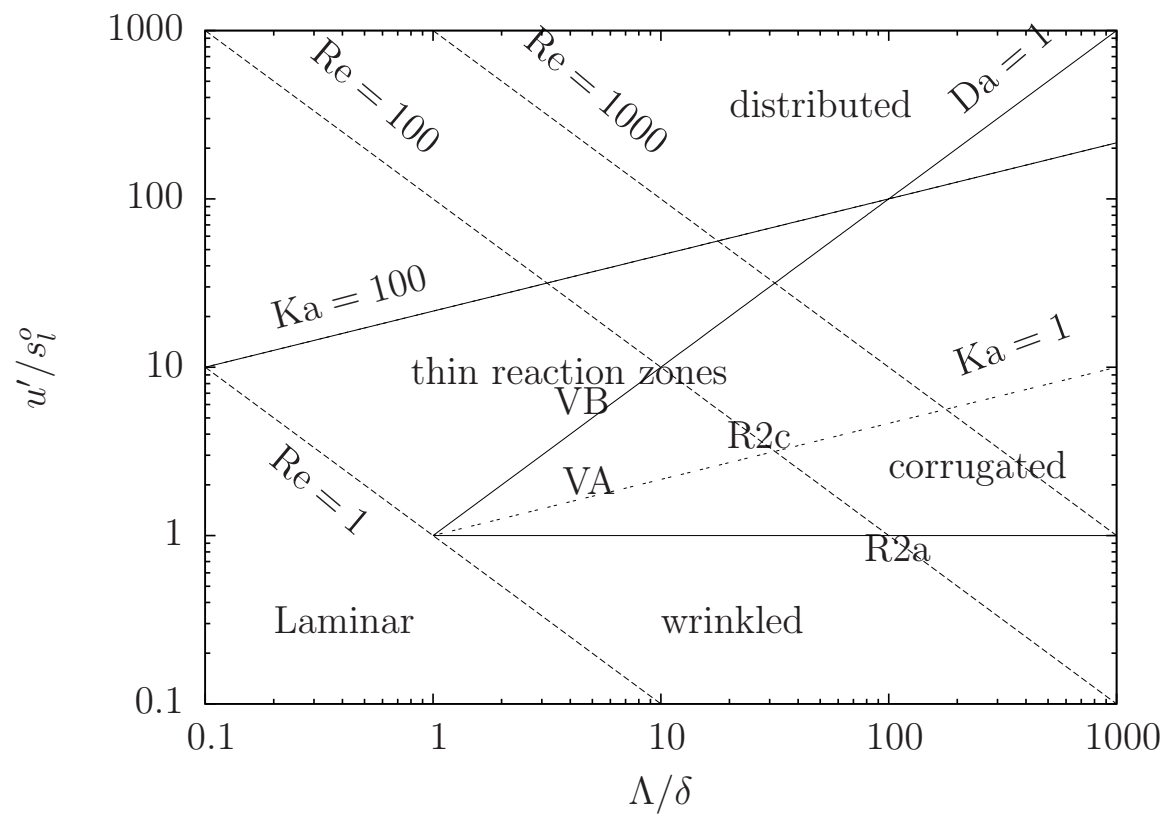

Figure 4.3: The premixed combustion regime diagram showing the selected DNS flames.

\subsubsection{Results and Discussion}

The code used to determine the solution for the minimal least squares norm, Eq. (4.11), is developed at Moscow State University [115]. The regularization parameter set to be 1.001 to give the smallest value of residual with 560 number of iterations. The initial solution is specified from the results obtained using algebraic model, Eq. (4.7).

Figures 4.4 and 4.5 show the variation of the conditional mean scalar dissipation rate, $\left\langle N_{\theta} \mid \zeta\right\rangle^{+}$, with sample space, $\zeta$, at $\tilde{\theta}=0.5$ for flames R2a and R2c and $\tilde{\theta}=0.6$ for $\mathrm{V}$ flames. The results are obtained using algebraic model, Eq. (4.7), and inverse model, Eq. (4.11), and compared to DNS results. The results obtained are normalised using $S_{L}^{o}$ and $\delta_{L}^{o}$. As shown in the figures, both the inverse model Eq. (4.11) and the algebraic model Eq. (4.7) have predicted the peak of conditional mean scalar dissipation rate. The values of $\left\langle N_{\theta} \mid \zeta\right\rangle^{+}$obtained by the 

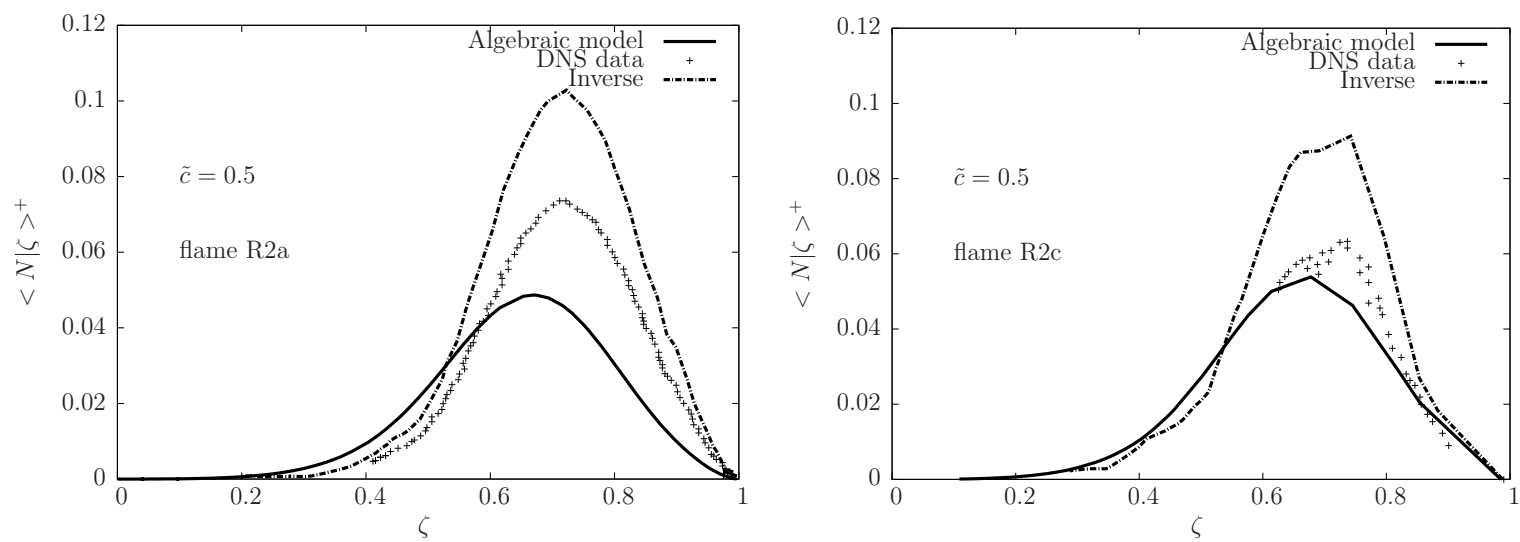

Figure 4.4: The typical variation of the conditional scalar dissipation rate, $\langle N \mid \zeta\rangle^{+}$, calculations obtained by, Eq. (4.7) and Eq. (4.11) with $\zeta$ in flames R2a and R2c. The values are non-dimensionalised using $S_{L}^{o}$ and $\delta_{L}^{o}$.

algebraic model are in good agreement with the DNS data for flame R2c and slightly under predicts flame R2a, while values obtained by the inverse model are over-prediction. As noted earlier, the inverse model requires an initial estimate for the conditional mean scalar dissipation rate and the model was found to be very sensitive to the initial estimate. For example if the initial estimate is increased by $30 \%$ then the model will deviate drastically from the DNS value. 

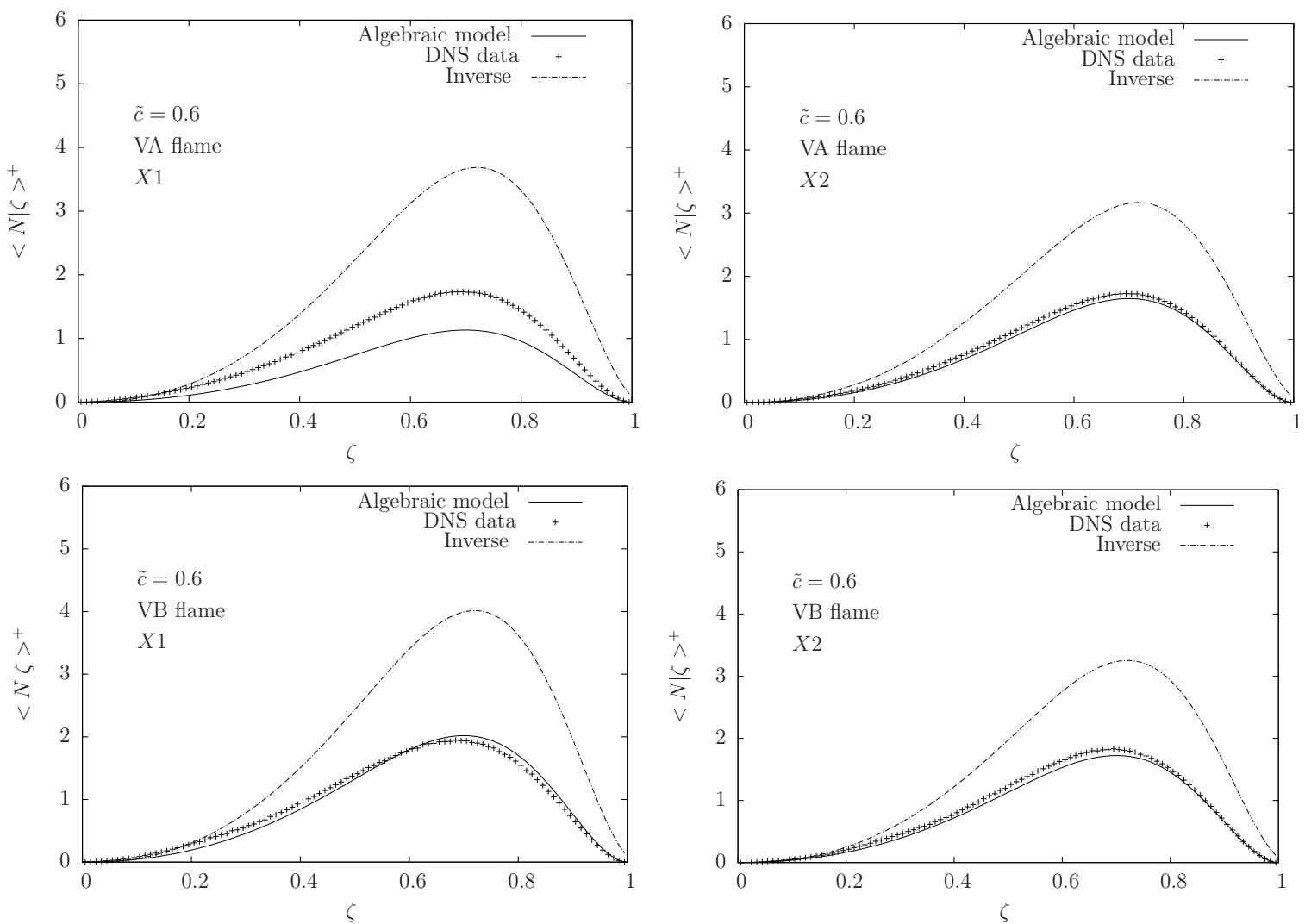

Figure 4.5: The typical variation of the conditional scalar dissipation rate, $\langle N \mid \zeta\rangle^{+}$, obtained by, Eq. (4.7) and Eq. (4.11) with $\zeta$ in flames VA and VB at two different axial locations in the flame; $x_{1}=16.7 \delta_{L}^{\circ}$ and $x_{2}=27.9 \delta_{L}^{\circ}$. These values are non-dimensionalised using $S_{L}^{o}$ and $\delta_{L}^{o}$. 


\section{Chapter 5}

\section{Mathematical Model}

This chapter presents a summary of the numerical methods used to simulate turbulent flows, since these methods are well established in the literature now. The discretisation of the CMC equation using the finite volume methodology is described in some detail, and this methodology follows an earlier study [32] where the CMC code for non-premixed flames was developed. The modifications to the code needed for premixed flames are described in section 5.2. The solution methodology and the interaction of RANS and CMC equations are described in section 5.3.

\subsection{Discretisation of the CMC Equation}

The stationary form of the governing equations given in section 2.3 for the mean fields are discretised by finite volume methodology of Patankar [116] using a power law scheme [116] for the spatial derivatives. In Cartesian coordinates $(x, y, z)$, the conservative form of CMC transport equation is discretised [32] over a physical 


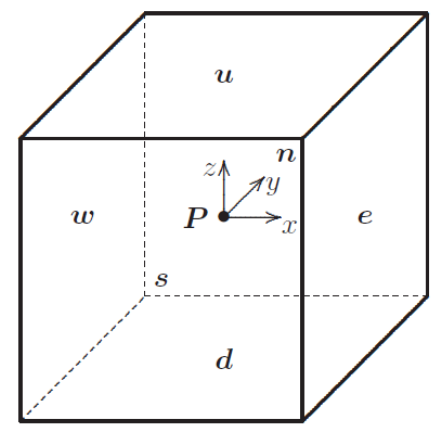

Real space

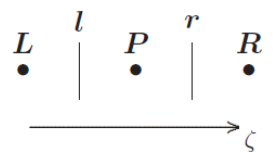

Progress variable space $\zeta$

Figure 5.1: Control volume in a finite volume method.

space control volume as shown in Fig. 5.1. The point $P$ is at the center of the control volume and the neighbouring cells are designated as west, east, south, north, up and down. The walls of the cells are represented by lower case letters. In the progress variable space, the neighbouring points are designated as left and right. The conservative form of Eq. (3.3) is written as

$$
\begin{array}{r}
\frac{\partial}{\partial x_{i}}\left(\gamma\left\langle u_{i} \mid \zeta\right\rangle Q_{\alpha}-\gamma D_{t} \frac{\partial Q_{\alpha}}{\partial x_{i}}\right)-\frac{\operatorname{Le}_{\theta}}{\operatorname{Le}_{\alpha}} \gamma\left\langle N_{\theta} \mid \zeta\right\rangle \frac{\partial^{2} Q_{\alpha}}{\partial \zeta^{2}}=\gamma\left\langle\dot{\omega}_{\alpha} \mid \zeta\right\rangle- \\
\frac{\partial}{\partial \zeta}\left[\gamma\left\langle\dot{\omega}_{\theta} \mid \zeta\right\rangle Q_{\alpha}\right],
\end{array}
$$

where, $\gamma=\langle\rho \mid \zeta\rangle \widetilde{p}(\zeta)$. Integrating equation Eq. (5.1) over the control volumes in real and progress variable spaces and dividing by $\gamma V \Delta \zeta$ yields

$$
\frac{\gamma_{e} A_{e}}{\gamma V}\left(\left\langle u_{i} \mid \zeta\right\rangle Q_{\alpha}-D_{t} \frac{\partial Q_{\alpha}}{\partial x}\right)_{e}-\frac{\gamma_{w} A_{w}}{\gamma V}\left(\left\langle u_{i} \mid \zeta\right\rangle Q_{\alpha}-D_{t} \frac{\partial Q_{\alpha}}{\partial x}\right)_{w}
$$




$$
\begin{array}{r}
+\frac{\gamma_{n} A_{n}}{\gamma V}\left(\left\langle u_{j} \mid \zeta\right\rangle Q_{\alpha}-D_{t} \frac{\partial Q_{\alpha}}{\partial y}\right)_{n}-\frac{\gamma_{s} A_{s}}{\gamma V}\left(\left\langle u_{j} \mid \zeta\right\rangle Q_{\alpha}-D_{t} \frac{\partial Q_{\alpha}}{\partial y}\right)_{s} \\
+\frac{\gamma_{u} A_{u}}{\gamma V}\left(\left\langle u_{k} \mid \zeta\right\rangle Q_{\alpha}-D_{t} \frac{\partial Q_{\alpha}}{\partial z}\right)_{u}-\frac{\gamma_{d} A_{d}}{\gamma V}\left(\left\langle u_{k} \mid \zeta\right\rangle Q_{\alpha}-D_{t} \frac{\partial Q_{\alpha}}{\partial z}\right)_{d} \\
-\frac{\operatorname{Le}_{\theta}}{\operatorname{Le}_{\alpha}} \frac{\left\langle N_{\theta} \mid \zeta\right\rangle}{\Delta \zeta}\left[\left(\frac{\partial Q_{\alpha}}{\partial \zeta}\right)_{r}-\left(\frac{\partial Q_{\alpha}}{\partial \zeta}\right)_{l}\right]=\frac{\gamma V}{\gamma V}\left\langle\dot{\omega}_{\alpha} \mid \zeta\right\rangle-\frac{\gamma V\left\langle\dot{\omega}_{\theta} \mid \zeta\right\rangle}{\gamma V \Delta \zeta}\left[Q_{r}-Q_{l}\right]
\end{array}
$$

The face values of the conditional quantities, $Q_{\alpha}$, in the advection terms of Eq. (5.2) are written as

$$
Q_{e}=f_{e} Q_{E}+\left(1-f_{e}\right) Q_{P}
$$

and

$$
Q_{w}=f_{w} Q_{W}+\left(1-f_{w}\right) Q_{P}
$$

where the subscripts $e$ and $w$ represent the east and west walls of the cell and the subscripts $E$ and $W$ represent the east and west neighbouring cells. The weighting factor $f$ is giving by

$$
f \equiv \frac{1}{P e}\left(1-(1-0.1 P e)^{5}\right)
$$

according to the power-law scheme of Patankar [116]. Accordingly, the total flux across each face in the positive and negative $x$-directions are

$$
\begin{aligned}
J_{e} & =\frac{\gamma_{e} A_{e}}{\gamma V}\left[\left\langle u_{i} \mid \zeta\right\rangle_{e} Q_{e}-\frac{D_{t}\left(Q_{E}-Q_{P}\right)}{(\delta x)_{e}}\right] \\
& =\frac{\gamma_{e} A_{e}}{\gamma V}\left[\left(Q_{E}-Q_{P}\right)\left(f_{e}\left\langle u_{i} \mid \zeta\right\rangle_{e}-\frac{D_{t}}{(\delta x)_{e}}\right)+\left\langle u_{i} \mid \zeta\right\rangle_{e} Q_{P}\right]
\end{aligned}
$$


and

$$
\begin{aligned}
J_{w} & =\frac{\gamma_{w} A_{w}}{\gamma V}\left[\left\langle u_{i} \mid \zeta\right\rangle_{w} Q_{w}-\frac{D_{t}\left(Q_{P}-Q_{W}\right)}{(\delta x)_{w}}\right] \\
& =\frac{\gamma_{w} A_{w}}{\gamma V}\left[\left(Q_{W}-Q_{P}\right)\left(f_{w}\left\langle u_{i} \mid \zeta\right\rangle_{w}-\frac{D_{t}}{(\delta x)_{w}}\right)+\left\langle u_{i} \mid \zeta\right\rangle_{w} Q_{P}\right]
\end{aligned}
$$

Defining the coefficients, $a_{E}, F_{e}, a_{W}$ and $F_{w}$ as

$$
\begin{gathered}
a_{E}=\frac{\gamma_{e} A_{e}}{\gamma V}\left(-f_{e}\left\langle u_{i} \mid \zeta\right\rangle_{e}+\frac{D_{t}}{(\delta x)_{e}}\right), \\
F_{e}=\frac{\gamma_{e} A_{e}\left\langle u_{i} \mid \zeta\right\rangle_{e}}{\gamma V}, \\
a_{W}=\frac{\gamma_{w} A_{w}}{\gamma V}\left(f_{w}\left\langle u_{i} \mid \zeta\right\rangle_{w}+\frac{D_{t}}{(\delta x)_{w}}\right),
\end{gathered}
$$

and

$$
F_{w}=\frac{\gamma_{w} A_{w}\left\langle u_{i} \mid \zeta\right\rangle_{w}}{\gamma V}
$$

The total fluxes in the positive and negative directions in the physical space are written as

$$
\begin{gathered}
J_{e}=a_{E}\left(Q_{P}-Q_{E}\right)+F_{e} Q_{P}, \\
J_{w}=a_{W}\left(Q_{W}-Q_{P}\right)+F_{w} Q_{P} .
\end{gathered}
$$

The same principle is used for the other faces.

In progress variable space, the advection terms of Eq. (5.2) are written as

$$
Q_{r}=f_{r} Q_{R}+\left(1-f_{r}\right) Q_{P}
$$




$$
Q_{l}=f_{l} Q_{L}+\left(1-f_{l}\right) Q_{P} .
$$

where the subscripts $L$ and $R$ represent the left and right neighbouring grid points in the progress variable space $\zeta$. Accordingly, the total fluxes are defined as

$$
\begin{aligned}
J_{r} & =\frac{\operatorname{Le}_{\theta}}{\operatorname{Le}_{\alpha}} \frac{\left\langle N_{\theta} \mid \zeta\right\rangle}{\Delta \zeta(\delta x)_{r}}\left(Q_{R}-Q_{P}\right)-\frac{\left\langle\dot{\omega}_{\theta} \mid \zeta\right\rangle}{\Delta \zeta} Q_{r} \\
& =\frac{\left(Q_{R}-Q_{P}\right)}{\Delta \zeta}\left(-f_{r}\left\langle\dot{\omega}_{\theta} \mid \zeta\right\rangle+\frac{\operatorname{Le}_{\theta}}{\operatorname{Le}_{\alpha}} \frac{\left\langle N_{\theta} \mid \zeta\right\rangle}{(\delta x)_{r}}\right)-\frac{\left\langle\dot{\omega}_{\theta} \mid \zeta\right\rangle}{\Delta \zeta} Q_{P}
\end{aligned}
$$

and

$$
\begin{aligned}
J_{l} & =\frac{\operatorname{Le}_{\theta}}{\operatorname{Le}_{\alpha}} \frac{\left\langle N_{\theta} \mid \zeta\right\rangle}{\Delta \zeta(\delta x)_{l}}\left(Q_{L}-Q_{P}\right)-\frac{\left\langle\dot{\omega}_{\theta} \mid \zeta\right\rangle}{\Delta \zeta} Q_{l} \\
& =\frac{\left(Q_{L}-Q_{P}\right)}{\Delta \zeta}\left(-f_{l}\left\langle\dot{\omega}_{\theta} \mid \zeta\right\rangle+\frac{\operatorname{Le}_{\theta}}{\operatorname{Le}_{\alpha}} \frac{\left\langle N_{\theta} \mid \zeta\right\rangle}{(\delta x)_{l}}\right)-\frac{\left\langle\dot{\omega}_{\theta} \mid \zeta\right\rangle}{\Delta \zeta} Q_{P}
\end{aligned}
$$

By defining the coefficients, $a_{R}$ and $a_{L}$ as

$$
\begin{aligned}
& a_{R}=\frac{1}{\Delta \zeta}\left(-f_{r}\left\langle\dot{\omega}_{\theta} \mid \zeta\right\rangle+\frac{\operatorname{Le}_{\theta}}{\operatorname{Le}_{\alpha}} \frac{\left\langle N_{\theta} \mid \zeta\right\rangle}{(\delta x)_{r}}\right), \\
& a_{L}=\frac{1}{\Delta \zeta}\left(-f_{l}\left\langle\dot{\omega}_{\theta} \mid \zeta\right\rangle+\frac{\operatorname{Le}_{\theta}}{\operatorname{Le}_{\alpha}} \frac{\left\langle N_{\theta} \mid \zeta\right\rangle}{(\delta x)_{l}}\right) .
\end{aligned}
$$

The total fluxes in the positive and negative directions of $\zeta$ co-ordinate are written as

$$
J_{r}=a_{R}\left(Q_{R}-Q_{P}\right)+\frac{\left\langle\dot{\omega}_{\theta} \mid \zeta\right\rangle Q_{P}}{\Delta \zeta}
$$


and

$$
J_{l}=a_{L}\left(Q_{P}-Q_{L}\right)+\frac{\left\langle\dot{\omega}_{\theta} \mid \zeta\right\rangle Q_{P}}{\Delta \zeta} .
$$

Now the total flux balance equation is written as

$$
\begin{array}{r}
J_{e}-J_{w}+J_{n}-J_{s}+J_{u}-J_{d}=\left(J_{r}\right)-\left(J_{l}\right)+\left\langle\dot{\omega}_{\alpha} \mid \zeta\right\rangle \\
+Q_{P}\left(F_{e}-F_{w}+F_{n}-F_{s}+F_{u}-F_{d}\right) .
\end{array}
$$

Substituting the total fluxes in Eq. (5.24), and after some arrangement yields the final discretised CMC transport equation, which is written as

$$
\sum a_{\mathrm{nb}} Q_{P}-\sum a_{\mathrm{nb}} Q_{\mathrm{nb}}=\left\langle\dot{\omega}_{\alpha} \mid \zeta\right\rangle .
$$

A first order closure of the chemical source term is obtained by linearising it with respect to $Q$. This algebraic equation is solved in the CMC part of the computer code.

\subsection{Implementation for Premixed Flames}

The original version of the research code was designed for non-premixed flames with two separate streams [117], fuel and oxidiser, with their mixing being described by solving a transport equation for Favre averaged mixture fraction, which is a passive scalar, see Eq. (2.29). In contrast, the pilot stabilised premixed flames involve more than two streams, the premixed reactant stream, the pilot stream and the ambient air stream. Note that, some cases can have more than one pilot stream, as noted in chapter 7. For these reasons, the code has been modified 
to include more than two streams along with appropriate transport equations to track the fluids emerging from these various streams. The transport equations for the Favre averaged progress variable $\tilde{\theta}$ and its variance $\widetilde{\theta^{\prime \prime 2}}$, Eqs. (2.28) and (2.30) are also included. Furthermore, one must also include additional variables which are passive scalars, to account for the mixing among streams. The simple mixing rules used in this study are explained while presenting results for the test flames in the following chapters. The source term, $\overline{\dot{\omega}}_{\theta}$, in Eq. (2.28) is closed in a manner consistent with CMC methodology as

$$
\overline{\dot{\omega}}_{\theta}=\int_{0}^{1}\left\langle\dot{\omega}_{\theta} \mid \zeta\right\rangle p d \zeta
$$

The dissipation rate, $\widetilde{\epsilon}_{\theta}$, of the variance $\widetilde{\theta^{\prime \prime 2}}$ in Eq. (2.30) is closed using Eq. (4.1) and the chemical source term is modelled using

$$
\overline{c^{\prime \prime} \dot{\omega}_{\theta}}=\int_{0}^{1} \zeta\left\langle\dot{\omega}_{\theta} \mid \zeta\right\rangle p d \zeta-\tilde{\theta} \int_{0}^{1}\left\langle\dot{\omega}_{\theta} \mid \zeta\right\rangle p d \zeta
$$

The solution to CMC equations requires initial conditions. For this purpose, steady flamelet equations written as

$$
\frac{\operatorname{Le}_{\theta}}{\operatorname{Le}_{\alpha}} \rho N_{\theta} \frac{\partial^{2} Y_{\alpha}}{\partial \zeta^{2}}+\dot{\omega}_{\alpha}-\dot{\omega}_{\theta} \frac{\partial Y_{\alpha}}{\partial \zeta}=0
$$

with appropriate boundary conditions at $\zeta=0$ and $\zeta=1$ for $Y_{\alpha}$, are solved. This equation is also implemented in the code for this purpose by comparing its solution with that obtained using Chemkin for a freely propagating laminar premixed flame. The variation of $N_{\theta}$ with $\theta$ obtained from Chemkin was supplied to this flamelet equation and the chemical kinetics was modelled using GRI-mech. 


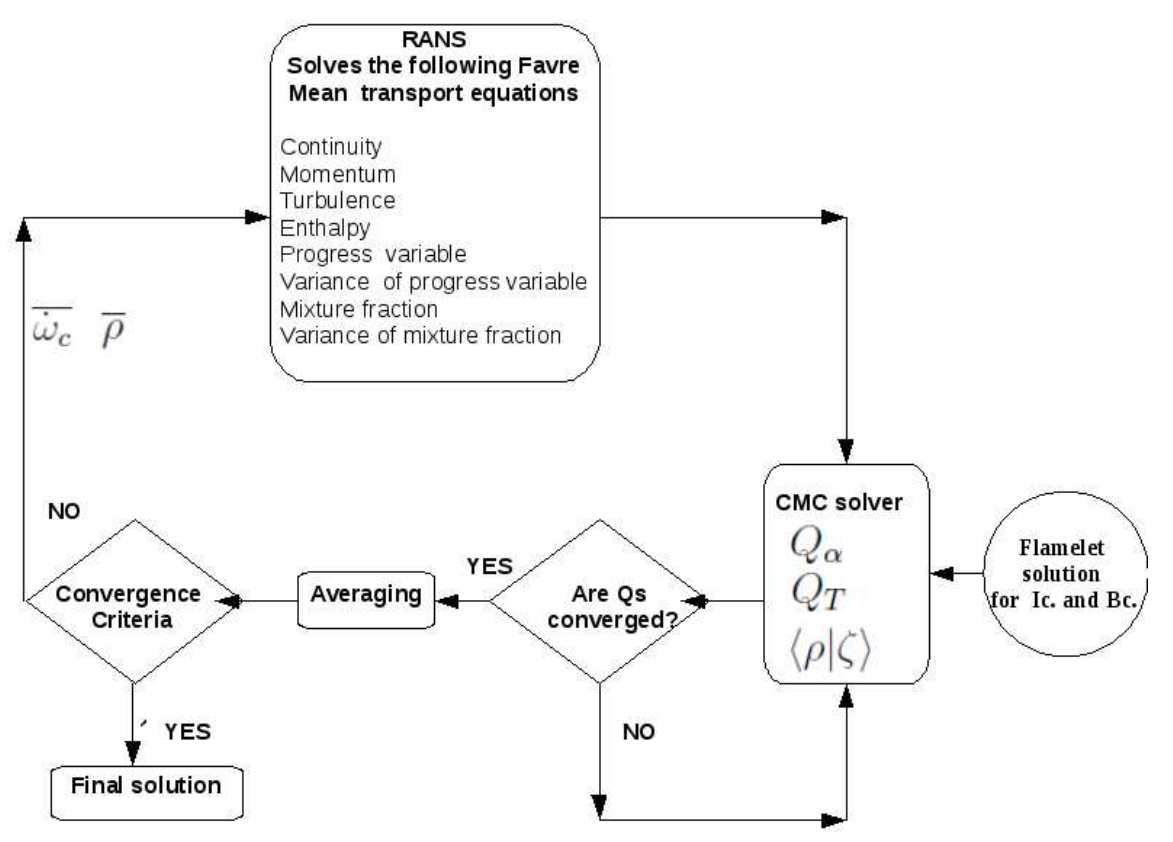

Figure 5.2: Interaction of CMC and RANS equation in the simulation.

3.0. The premixed CMC transport equation is closed using the models discussed in section 3.3

\subsection{Computer Model and Sequence}

The Favre averaged, continuity, momentum and enthalpy equations are solved along with transport equations for $\tilde{\theta}, \widetilde{\theta^{\prime \prime^{2}}}, \tilde{Z}, \tilde{k}$ and $\tilde{\varepsilon}$.

The flow chart shown in Fig. 5.2 describes the computational sequence and the interaction between RANS and CMC equations. A SIMPLER approach [116] is used to couple the velocity and pressure fields inside the computational domain. These discretised equations are solved using an iterative algorithm using under relaxation factors, given in table 5.1. The mean flow and turbulence quantities obtained from the fluid dynamics solver are passed to the CMC solver. A steady 


\begin{tabular}{cccccccccccccc}
\hline$P$ & $u$ & $v$ & $w$ & $k$ & $\varepsilon$ & $h$ & $\mu$ & $\rho$ & $T$ & $\tilde{\theta}$ & $\widetilde{\theta^{\prime \prime 2}}$ & $\tilde{Z}$ & $\widetilde{Z^{\prime \prime 2}}$ \\
\hline 0.4 & 0.4 & 0.4 & 0.4 & 0.4 & 0.4 & 0.4 & 0.4 & 0.4 & 0.1 & 0.1 & 0.1 & 0.1 & 0.1 \\
\hline
\end{tabular}

Table 5.1: Under-relaxation factors.

flamelet solution is used to specify the initial and the boundary conditions for the CMC equations as noted in section 5.2, and the CMC equations are solved to get a converged solution. Then the mean density, $\overline{\dot{\omega}}_{\theta}$ and $\overline{\theta^{\prime \prime} \dot{\omega}_{\theta}}$ calculated using the respective conditional means obtained from the CMC solver are fed back to the fluid dynamics part. These processes are iterated, until convergence criteria are met for all quantities of interest as shown in Fig. 5.2. This convergence criteria is set to be 5E-5 for the fluid dynamics and CMC. Further detail on the computational tool used in this study can be found in $[32 ; 117]$ as that study validated this tool for bagasse-fired boiler involving a non-premixed flame.

From the converged solution, the various mean quantities, required for comparison with experimental measurements, are obtained as

$$
\widetilde{Y_{\alpha}}=\int_{0}^{1} Q_{\alpha} \widetilde{p} d \zeta
$$

The computational results obtained using the numerical methods described above are compared to experimental measurement of pilot stabilised turbulent premixed flames for range of thermochemical and turbulence conditions. These comparisons are discussed in the following chapters. 


\section{Chapter 6}

\section{Stoichiometric Premixed Flame}

\section{Calculation}

\subsection{Computational Details}

The test flames used in this chapter are the pilot stabilised turbulent Bunsen flames of Chen et al. [2], which is shown schematically in Fig. 6.1 along with the computational domain and boundary conditions used. These flames have been investigated in earlier studies to validate various premixed turbulent combustion models such as transported PDF model [68; 118], flame surface density approach [119], strained flamelets [79] and G-equation [120]. Recently, these flames have also been computed using large eddy simulation with the thickened flame model [121]. Stoichiometric methane-air flames with a fuel nozzle diameter $D=12 \mathrm{~mm}$ and a pilot diameter $d_{p}=68 \mathrm{~mm}$ were considered. Three flames designated as F1, F2, and F3, were investigated in the experiments. The bulk mean velocity, $U_{o}$, at the exit of the fuel nozzle is 65,50 and $30 \mathrm{~m} / \mathrm{s}$ for flames F1, F2 and F3 


\section{Stoichiometric Premixed Flame Calculation}

respectively. The Reynolds number, Re, based on $D$ and $U_{o}$, for these flames are 52000, 40000 and 24000 respectively. The hot stoichiometric products from the pilot flames were flowing with a uniform velocity of $0.2 \mathrm{~m} / \mathrm{s}$, and this value is obtained from the reported [2] volumetric flow rate at the burner exit.

As shown in the turbulent combustion regime diagram, Fig. 6.2, flames F1 and F2 are in the thin reaction zones regime and flame F3 is on the border between the thin reaction zones and the flamelets regimes. In the current study these flames are computed using the RANS-CMC approach, with a specific interest on carbon monoxide since the suggested [40] contribution of the CMC for premixed systems is expected to be in the prediction of species with slow time scales including pollutants.

Since no explicit assumptions on the influence of turbulent eddies on the flame front structure were made while deriving the CMC equation, Eq. (3.3), the CMC may hold in all regimes of premixed combustion although its successful application would be determined by the accuracy of the closure models and the assumptions used to obtain them. The conditional scalar dissipation rate, $\left\langle N_{c} \mid \zeta\right\rangle$, is important from this view-point since the progress variable gradient is strongly determined by chemical reaction, molecular diffusion and their interaction in premixed flames. However, as [40] noted, the contribution of CMC for premixed combustion would be on the prediction of species with slow time scales, including the effects of turbulence on the chemical structure. Using one time or length scale related to the fast chemical process, typically used to construct the regime diagram, may be insufficient to argue the applicability of the $\mathrm{CMC}$ in various premixed combustion regimes. Careful studies using DNS data with complex chemical kinetics in three dimensions and laser diagnostics data on the progress variable 

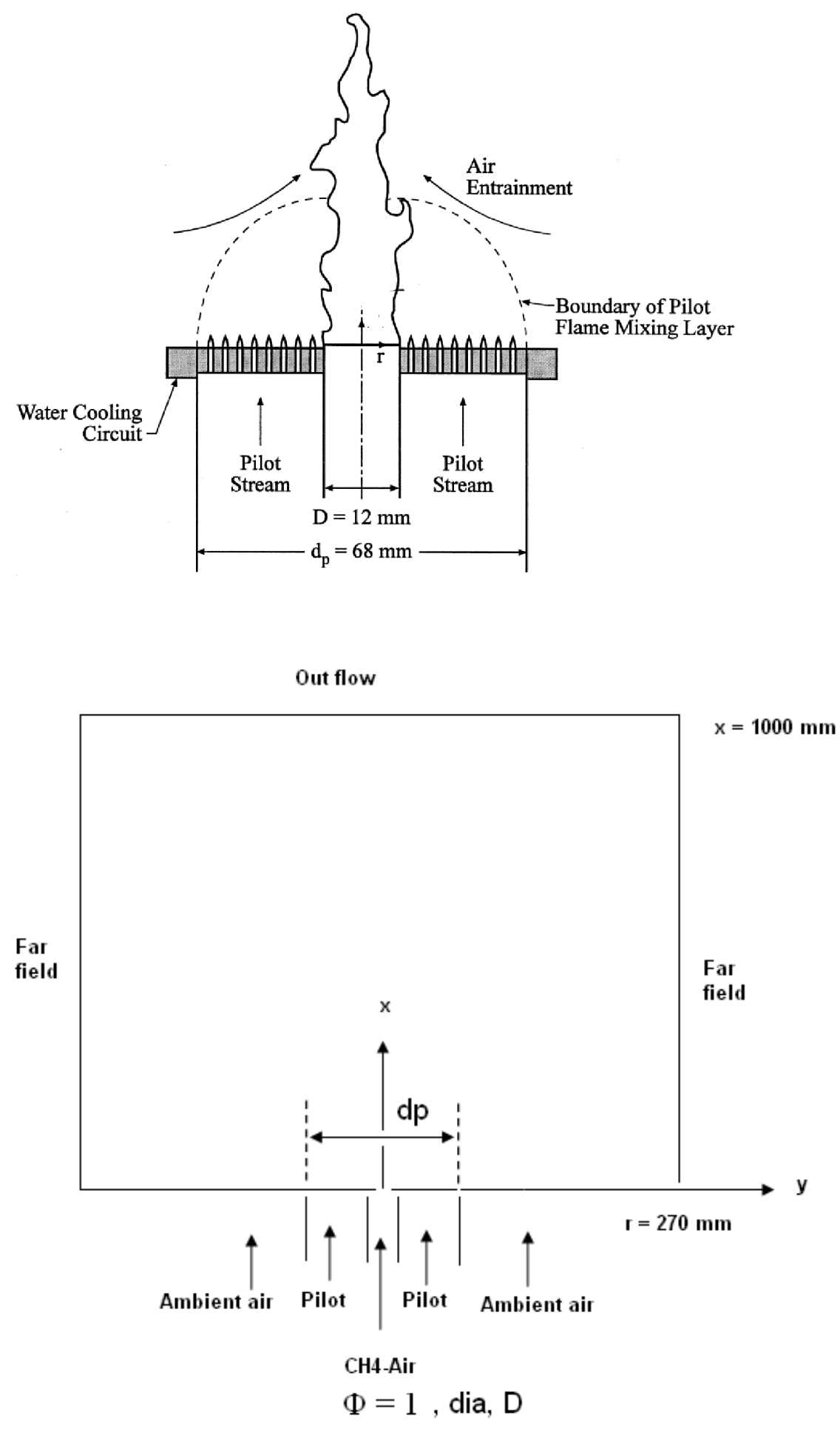

Figure 6.1: Schematic diagram of the burner setup [2] and the computational domain along with BCs. 


\section{Stoichiometric Premixed Flame Calculation}

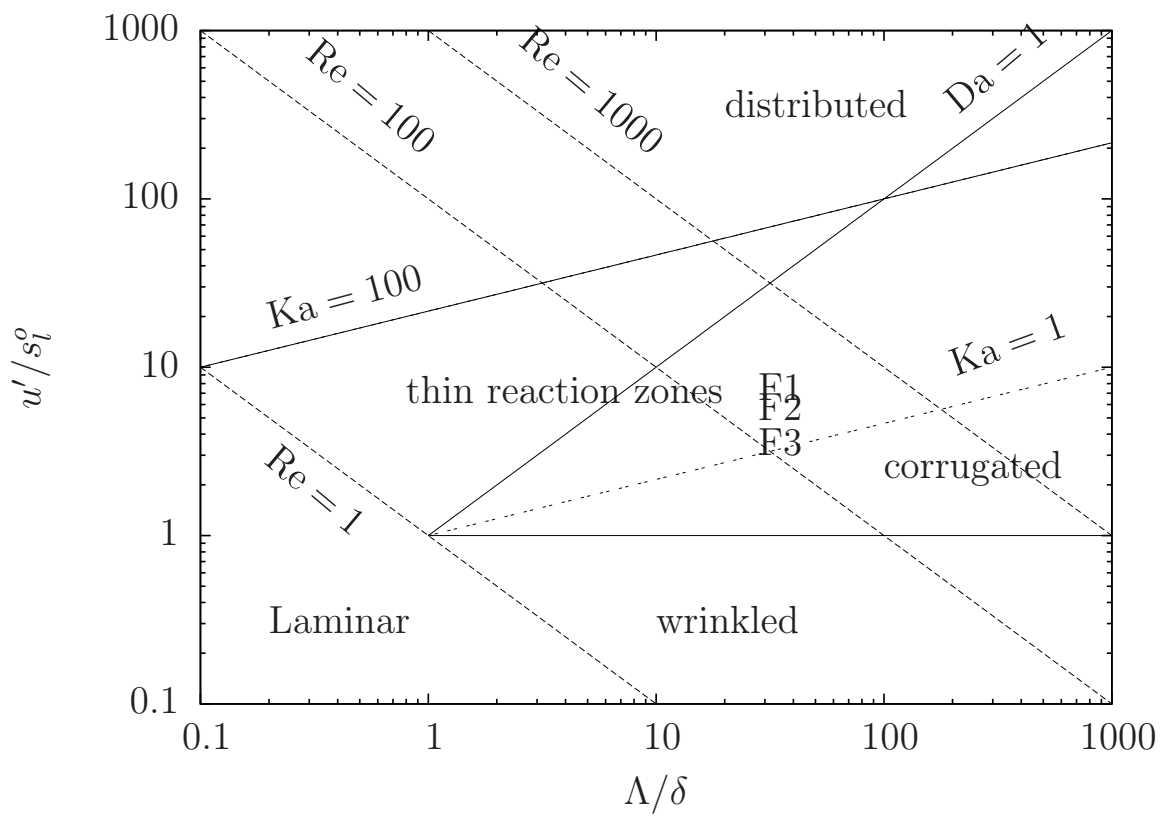

Figure 6.2: The premixed combustion regime diagram showing flames F1, F2 and F3.

gradient information and multi-species measurements are very much required to address this open question.

The Favre averaged, continuity, momentum and enthalpy equations are solved along with transport equations for $\tilde{\theta}, \widetilde{\theta^{\prime \prime^{2}}}, \tilde{Z}$ and $\widetilde{Z^{\prime \prime 2}}$. Although the mixture fraction variance is included, for the calculation of partially premixed flames in future, its solution is not used in this study. The mean mixture fraction must be seen as a marker for the reacting mixture from the main jet and pilot, and it is set to be unity in these two streams at the burner exit. This marker is used to account for the mixing between the jet fluid and surroundings air as discussed by [79]. The value of the total enthalpy computed using its transport equation at a grid point is written as

$$
\widetilde{h}=c_{p, \text { mix }}\left(\widetilde{T}-T_{o}\right)+\Delta h_{f, \text { mix }}^{o}
$$


where $c_{p, \text { mix }}=\widetilde{Z} \sum \widetilde{Y}_{\alpha} \theta_{p, \alpha}+(1-\widetilde{Z}) \theta_{p, \text { air }}$, and $\Delta h_{f, \text { mix }}^{o}=\widetilde{Z} \sum \widetilde{Y}_{\alpha} \Delta h_{f, \alpha}^{o}+(1-$ $\widetilde{Z}) \Delta h_{f \text {,air }}^{o}$, to obtain the average temperature $\widetilde{T}$. The enthalpy of formation for the air is taken to be zero.

The turbulence is modeled using a standard $k-\varepsilon$ model with changes to $C_{\epsilon 1}$ (from 1.44 to 1.52 ) to account for round jet anomaly [122]. The boundary conditions reported by [2] for the mean flow and turbulence quantities are used at the burner exit (see Fig. 6.1) for the calculations reported in this study. A small uniform velocity of $0.2 \mathrm{~m} / \mathrm{s}$ is specified to account for the ambient air entrainment. The mean progress variable is specified to be one in the main jet and zero in the pilot and ambient air streams. The variance of the progress variable is specified to be zero in all the streams entering the computational domain. The conditions at other computational boundaries are marked in Fig. 6.1.

A computational domain of size $1000 \mathrm{~mm}$ in the axial direction, $x$, and $270 \mathrm{~mm}$ in the radial direction, $r$, is used with refined grid near the fuel exit. The smallest cell size is $0.5 \times 1.0$ (in mm) and the CFD computational domain has $80 \times 80 \times 120$ cells in $y, z, x$ directions, for turbulence and mean quantities. For the CMC calculations, 500 non-uniform cells are used in $\zeta$ space to resolve the very strong gradients near the hot side ( $\zeta=0$ in Figs. 6.8 and 6.9 to be discussed later) and to obtain meaningful and smooth variations of conditional averages of major and minor species involved in the chemical mechanism. Two cells in each physical direction of the main grid for the fluid dynamics variables are combined to create the physical space grid for the CMC equations, since the conditional averages are expected to vary slowly in the physical space. These grids gave solutions with negligible sensitivity to any further changes in the physical or CMC grids. Thus, the results reported here are for the above grids. 
In order to assess the influence of chemical kinetics mechanism and $e_{Q_{\alpha}}$ term on the reacting flow results, three simulations were performed for flame F1 using two different chemical mechanisms for methane-air combustion. In the first case, the GRI-mechanism [123] is used, which consists of 325 reactions and 53 species. In the second case, the skeletal mechanism of [124] involving 25 elementary chemical reactions and 16 species is used. The effect of chemical kinetics mechanism is studied by comparing these two cases. To evaluate the influence of $e_{Q_{\alpha}}$, see Eq. (3.3), on the evolution of $Q_{\alpha}$, a third simulation is conducted using the GRImech and including $e_{Q_{\alpha}}$ given in Eq. (3.10). The other two flames, F2 and F3, are simulated using the GRI-mechanism excluding the contributions from $e_{Q_{\alpha}}$.

A steady flamelet solution is used to specify the initial and the boundary conditions for the $\mathrm{CMC}$ equations as noted in section 5.2. A single three-dimensional simulation took about 336 hours wall clock time in a $3.5 \mathrm{GHz}$, 8GB RAM, intel Xeon desktop computer to get a converged solution. This computational time can be reduced significantly by using parallel computation techniques, which are yet to be implemented in this computer code. The computed solutions are compared to experimental data reported by [2]. These comparison are discussed next.

\subsection{Results and Discussion}

\subsubsection{Non Reacting Flow}

The non-reacting flow were simulated first to assess the turbulence models and the flow boundary conditions used. Figures 6.3 and 6.4 show the normalised mean velocity and turbulence kinetic energy results in non-reacting flow for flames F1, 


\section{Stoichiometric Premixed Flame Calculation}

F2 and F3. The turbulence kinetic energy, $\widetilde{k}_{o}$, at the centre line of the fuel nozzle exit are 12.7, 10.8 and $3.82 \mathrm{~m}^{2} / \mathrm{s}^{2}$ for flames $\mathrm{F} 1, \mathrm{~F} 2$ and $\mathrm{F} 3$ respectively. These results are shown for five axial locations, the symbols are the experimental measurements and the lines are the computational results. The good agreement shown here for the mean velocity suggests that the spread rate of the cold jet is captured in the simulations and the boundary conditions used describe the experimental conditions well.

The normalised mean turbulence kinetic energy profiles for flame F1 are in excellent agreement with the measurements at the centre line for locations close to the nozzle exit, while further down-stream, it is slightly over predicted. In the radial direction, the computed $\tilde{k}$ is slightly over predicted at all locations in flame F1.

Almost similar levels of agreement in the mean velocity and turbulent kinetic energy are observed for flames F2 and F3 as shown in figures 6.3 and 6.4. The normalised mean turbulent kinetic energy agrees well with the measurements at the center line for all axial locations. In the radial directions, it is in good agreement with the measurements for all axial locations except, at location $x / d=10.5$, it is slightly over predicted in the radial direction. In general, the results from the cold flow are typical for $k-\varepsilon$ model, and they are satisfactory and acceptable.

The radial variation of $\widetilde{Z}$ at different axial distances is shown in Fig. 6.5 for flame F1. Unfortunately, there is no experimental data for this comparison, however, the spread of this marker variable is as expected in this type of flow.

Since $\widetilde{Z}=1$ in the fuel jet and pilot, the center-line value does not drop from unity for the stream-wise locations noted. 


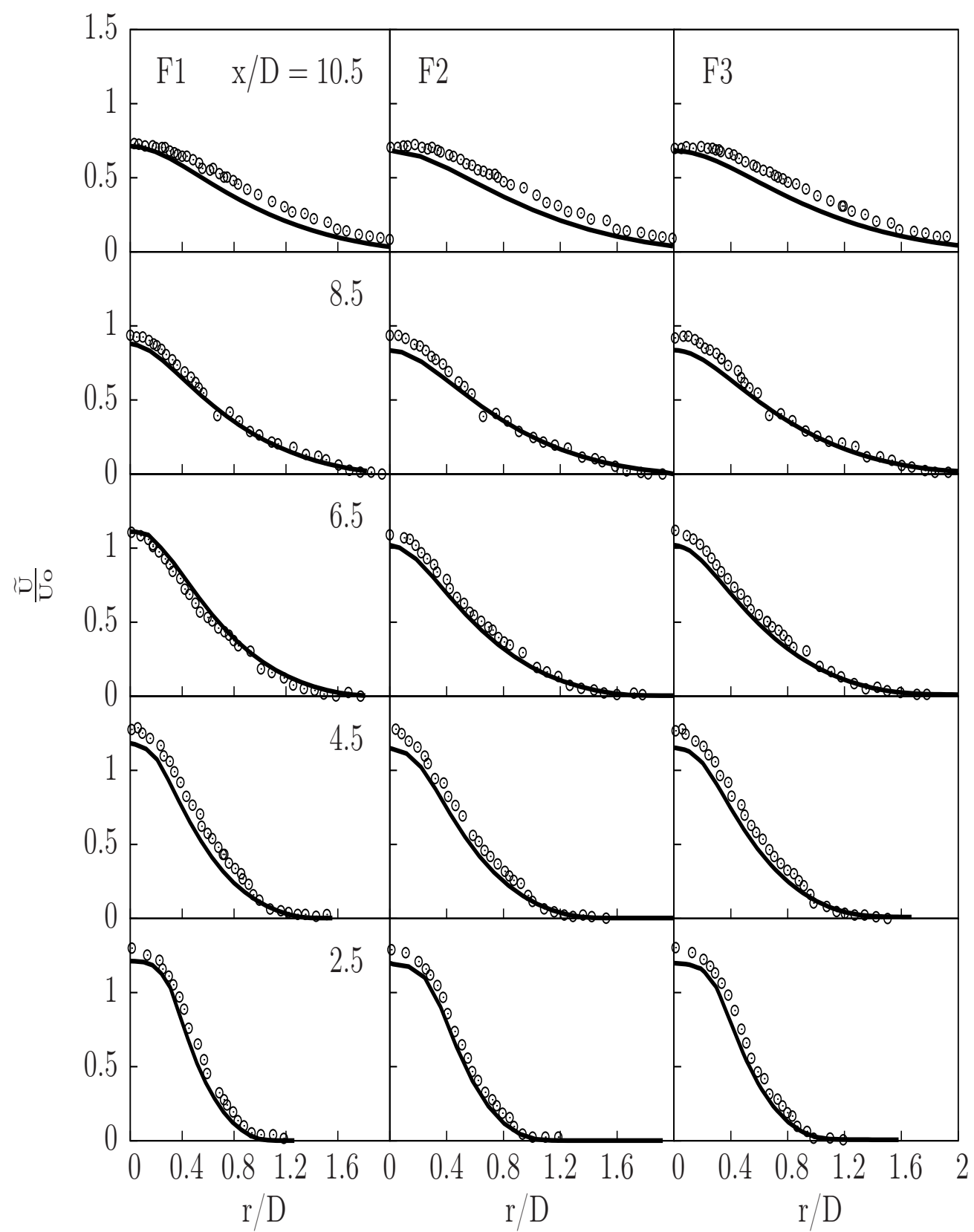

Figure 6.3: The normalised mean velocity (lines) from non-reacting flow are compared to the the experimental measurements (symbols) for flames F1, F2 and F3. 


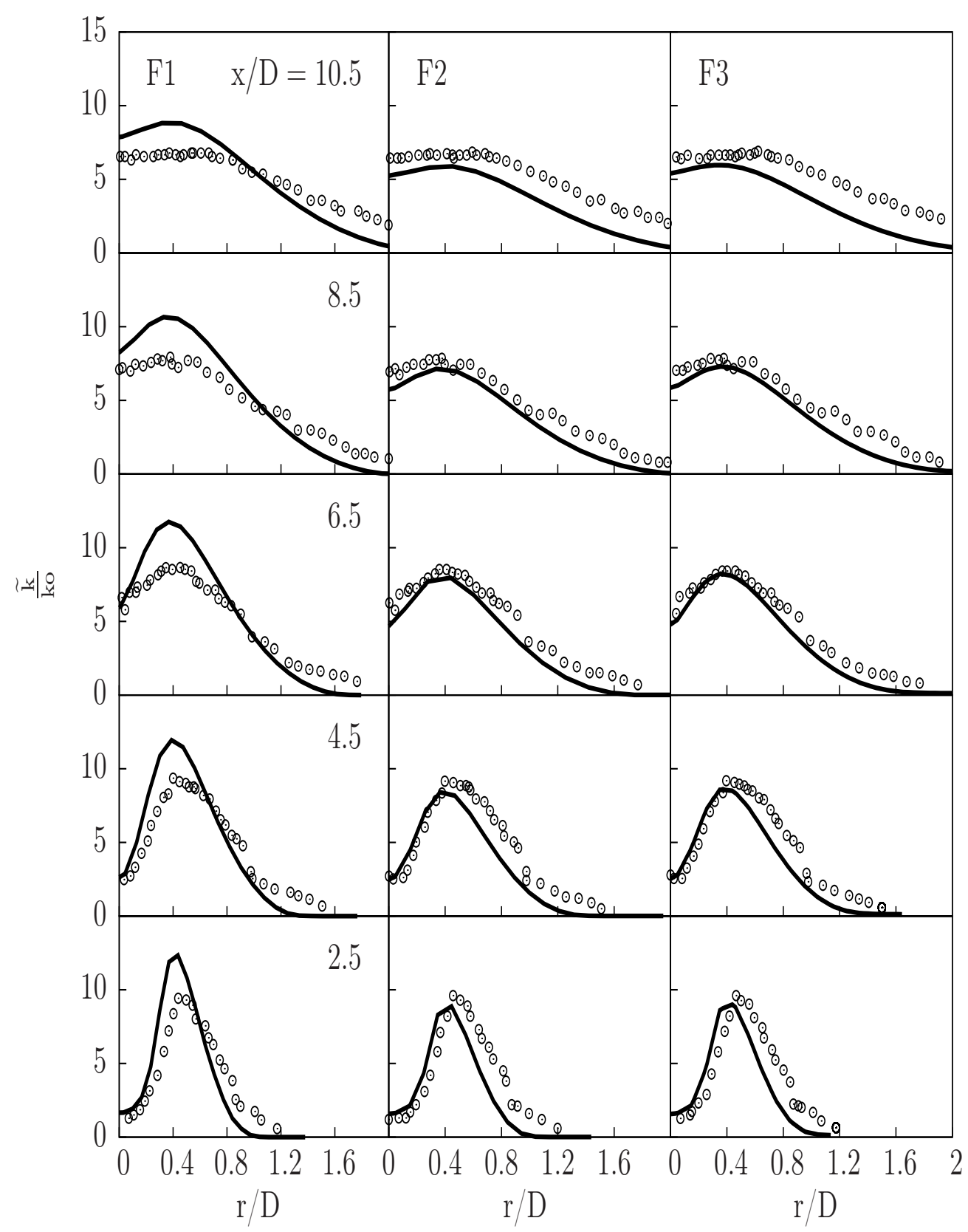

Figure 6.4: The normalised mean turbulent kinetic energy (lines) from nonreacting flow are compared to the experimental measurements (symbols) for flames F1, F2 and F3. 


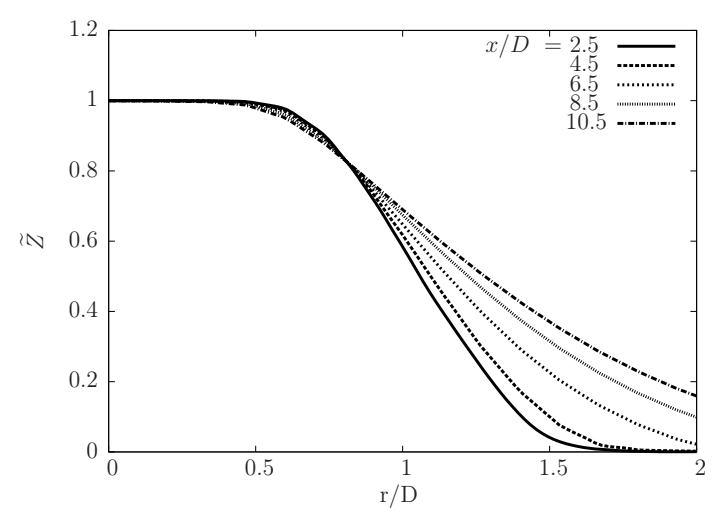

Figure 6.5: Computed radial variation of $\widetilde{Z}$ in cold flow simulation at five axial locations for the conditions of flame F1.

\subsubsection{Conditional Scalar Dissipation Rate}

The typical values of conditional dissipation rate obtained in the CMC calculation of flame F1 are shown in Figs. 6.6 and 6.7. Note that the model used in this calculation is the algebraic model, Eq. 4.7. These values obtained at axial locations $x / D=4.5$ and 8.5 for different $\widetilde{\theta}$ inside the flame brush. these values are non-dimensionalised using $S_{L}^{o}$ and $\delta_{L}^{o}$. As shown in the figure, the characteristics variation of $\langle N \mid \zeta\rangle^{+}$with $\zeta$ is observed in the CMC calculation. This is because the chemical reactions occur towards the hot side of the flame front, which is denoted by low value of $\zeta$, the progress variable. Unfortunately, there is no experimental data for this comparison, however, if one compares the values of $\langle N \mid \zeta\rangle^{+}$of flame F1 to the DNS flames shown in chapter 4, these values appear to be larger because the turbulence level in flame F1 is large compared to the DNS flames. Also as seen from the results the variation of $\langle N \mid \zeta\rangle^{+}$with different $\widetilde{\theta}$ and axial locations are very small. 


\section{Stoichiometric Premixed Flame Calculation}
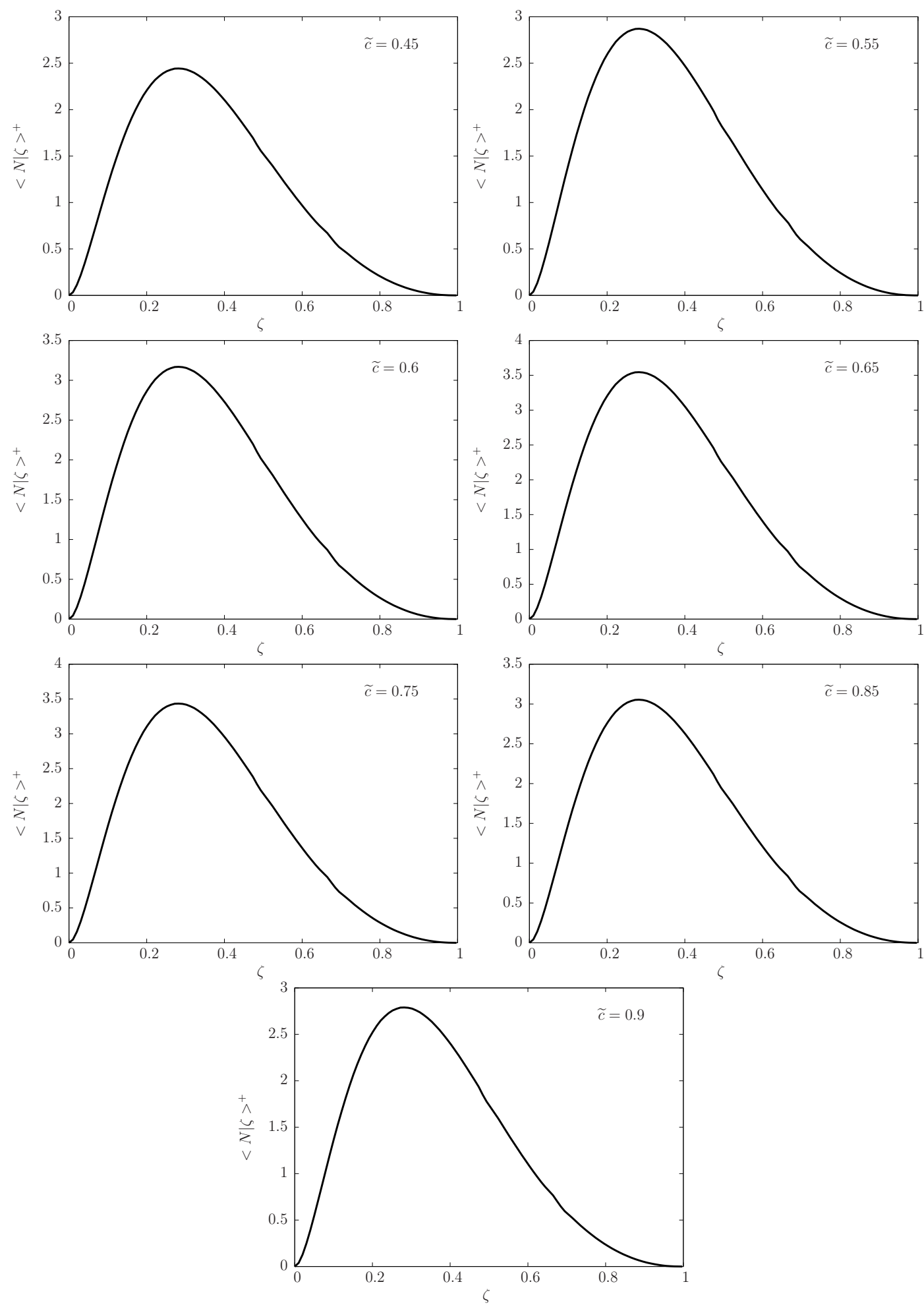

Figure 6.6: The typical variation of the conditional scalar dissipation rate, $\langle N \mid \zeta\rangle^{+}$, obtained in CMC calculations by, Eq. (4.7), with $\zeta$ in flame F1 at different locations inside the flame brush at $x / D=4.5$. The values are non-dimensionalised using $S_{L}^{o}$ and $\delta_{L}^{o}$. 


\section{Stoichiometric Premixed Flame Calculation}
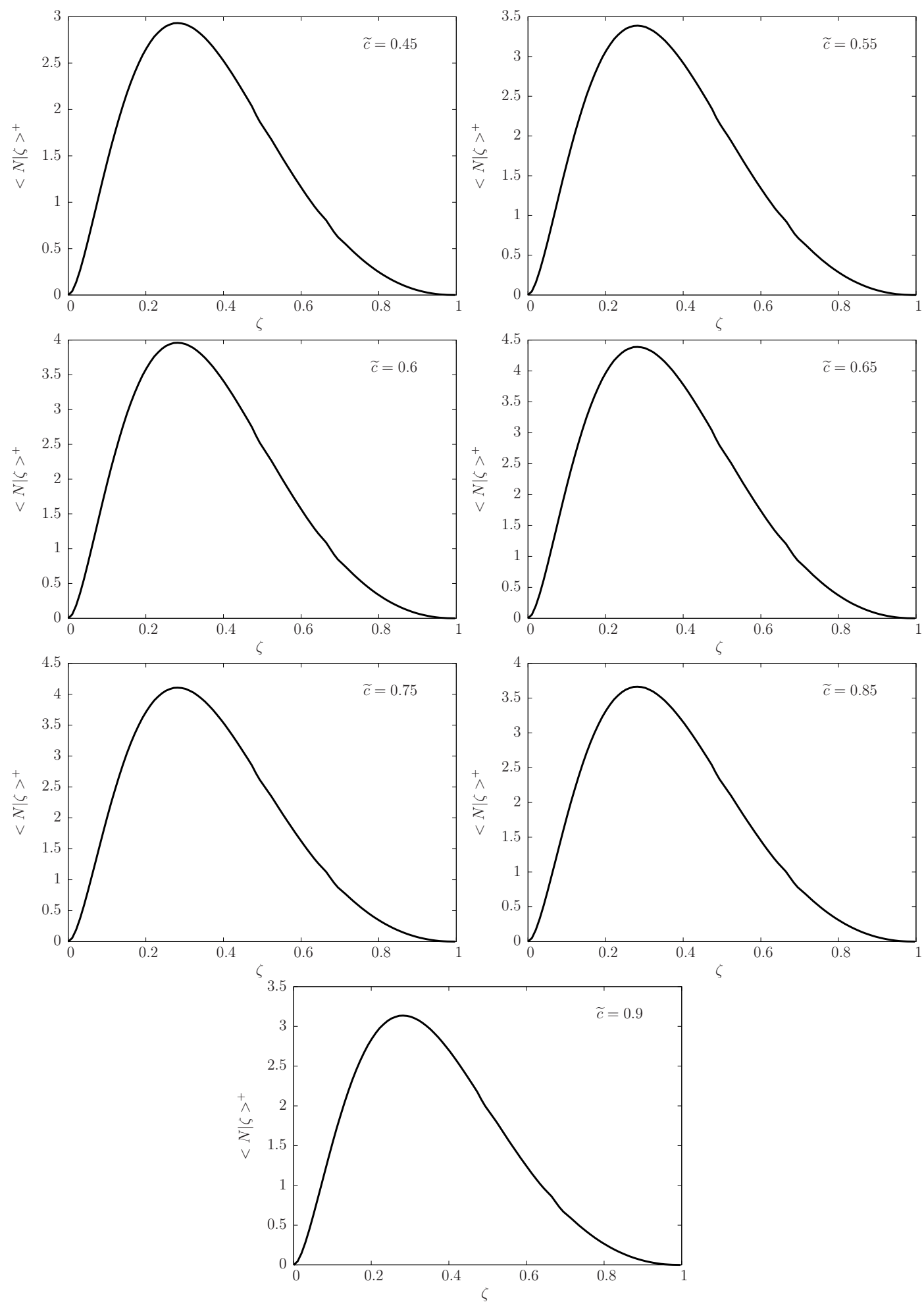

Figure 6.7: The typical variation of the conditional scalar dissipation rate, $\langle N \mid \zeta\rangle^{+}$, obtained in CMC calculations by, Eq. (4.7), with $\zeta$ in flame F1 at different locations inside the flame brush at $x / D=8.5$. The values are non-dimensionalised using $S_{L}^{o}$ and $\delta_{L}^{o}$. 


\subsubsection{Terms in CMC Transport Equation}

After neglecting the unsteady term in Eq. (3.3), the steady CMC transport equation can be written as

$$
\text { Conv. }- \text { Diff. }- \text { reac. }+\operatorname{reac}_{\theta}-\mathrm{e}_{\mathrm{Q}_{\alpha}}=\text { Imb. }
$$

The typical variation of these terms with $\zeta$ for a few species is shown in Fig. 6.8 using GRI-mech. The results are shown for few representative scalars from flame F1 at $\tilde{\theta}=0.55$ and $x / d=4.5$. Note that $\zeta=0$ is the burnt side and $\zeta=1$ is the unburnt side. Since the values shown in Fig. 6.8 do not include the algebraic sign in Eq. (6.2), a positive value indicates a source and a negative value implies a sink for the terms related to the reactions and $e_{Q_{\alpha}}$. Since the four species shown in Fig. 6.8 are produced by the chemical reactions in methane-air flames, the values of the "reac." term is positive. The reactive nature of the conditioning variable is making a sink contribution to these scalars. Also as observed in Fig. 6.8, there is a predominant balance between the convective and the diffusive terms and reaction rate of species $\alpha$ is balanced by the "reac ${ }_{\theta}$ " and $e_{Q_{\alpha}}$ terms in the reactive zone. The Lewis number for the conditioning variable is unity. The magnitude of $e_{Q_{\alpha}}$ is dictated by $\partial Q_{\alpha} / \partial \zeta$ and the value of Lewis number for species $\alpha$, see Eq. (3.10). However, if $\mathrm{Le}_{\alpha}$ is close to unity then one can see that $e_{Q_{\alpha}}=0$ unless $\partial Q_{\alpha} / \partial \zeta$ is singular. The negligible value of $e_{Q_{\alpha}}$ is noted in Fig. 6.8 for CO, since Le $\mathrm{CO}$ $=1.084$. The contribution of $e_{Q_{\alpha}}$ for $\mathrm{H}_{2} \mathrm{O}$ is smaller then the reactive terms but non-negligible because $\mathrm{Le}_{\mathrm{H}_{2} \mathrm{O}}=0.78$. However, for the atomic and molecular hydrogen the contribution of $e_{Q_{\alpha}}$ is as big as the reactive term in Eq. (3.10) since the Lewis number for these species are small $\left(\mathrm{Le}_{\mathrm{H}}=0.172, \mathrm{Le}_{\mathrm{H}_{2}}=0.293\right)$. Finally 

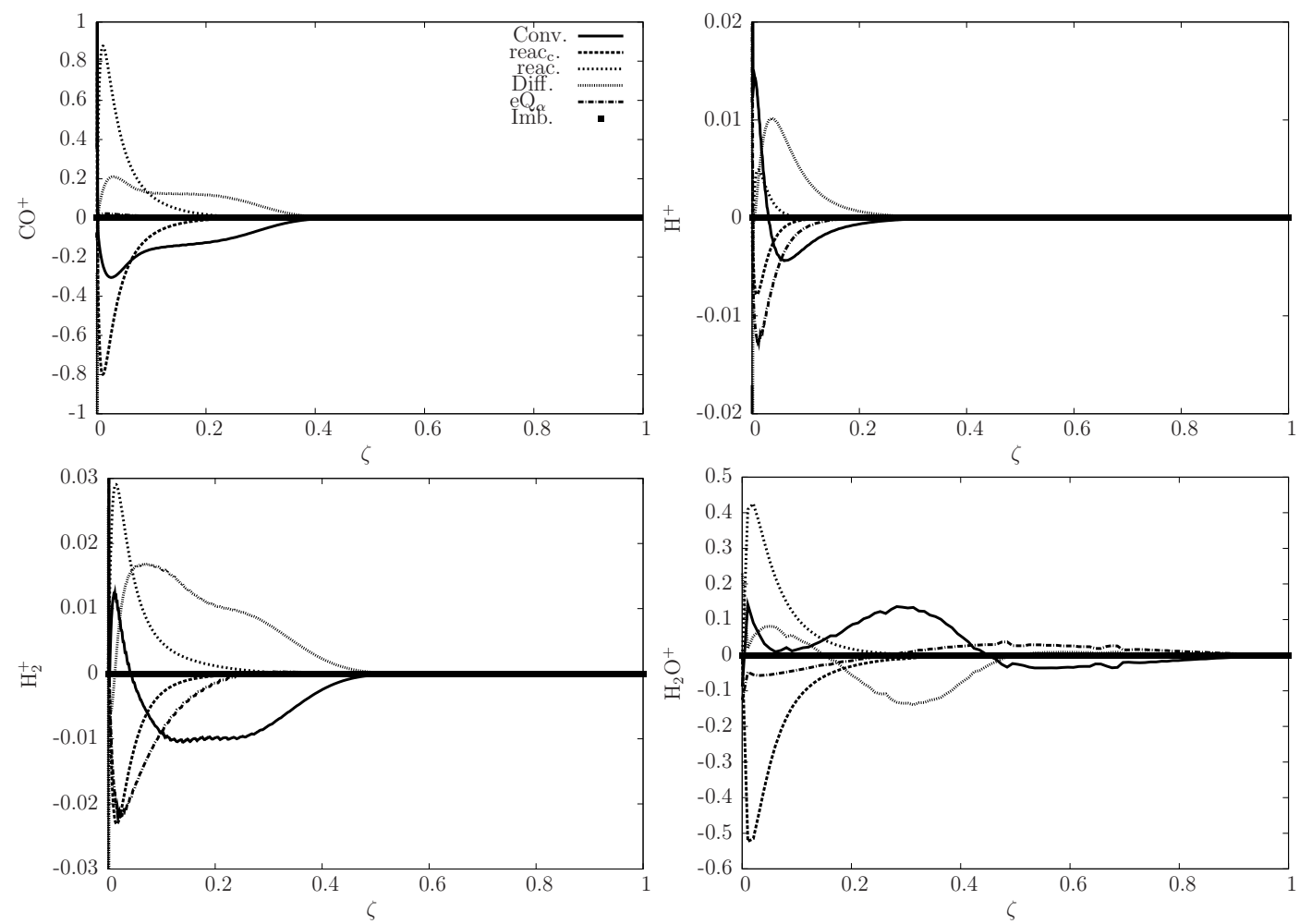

Figure 6.8: Typical variation of different terms of the CMC transport equation, Eq. (6.2), with $\zeta$ in flame F1 at $\tilde{\theta}=0.55$. The results are shown for few representative scalars. 

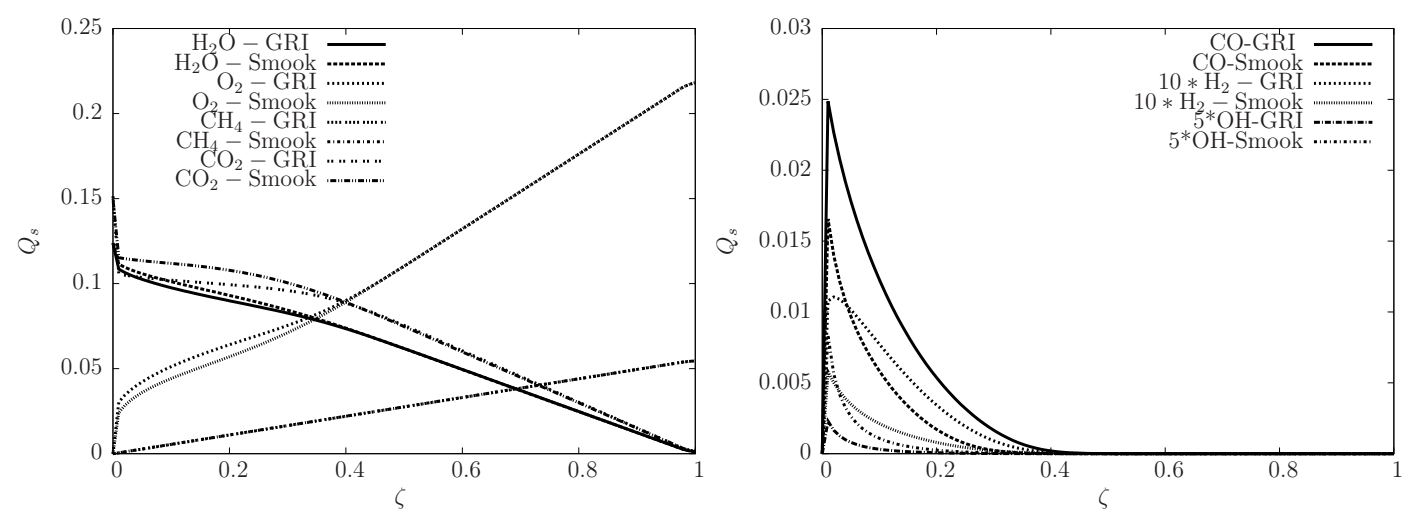

Figure 6.9: The variation of the conditional mean mass fraction with $\zeta$ for major and minor species in flame $\mathrm{F} 1$ at $\tilde{\theta}=0.55$ and $x / d=4.5$.
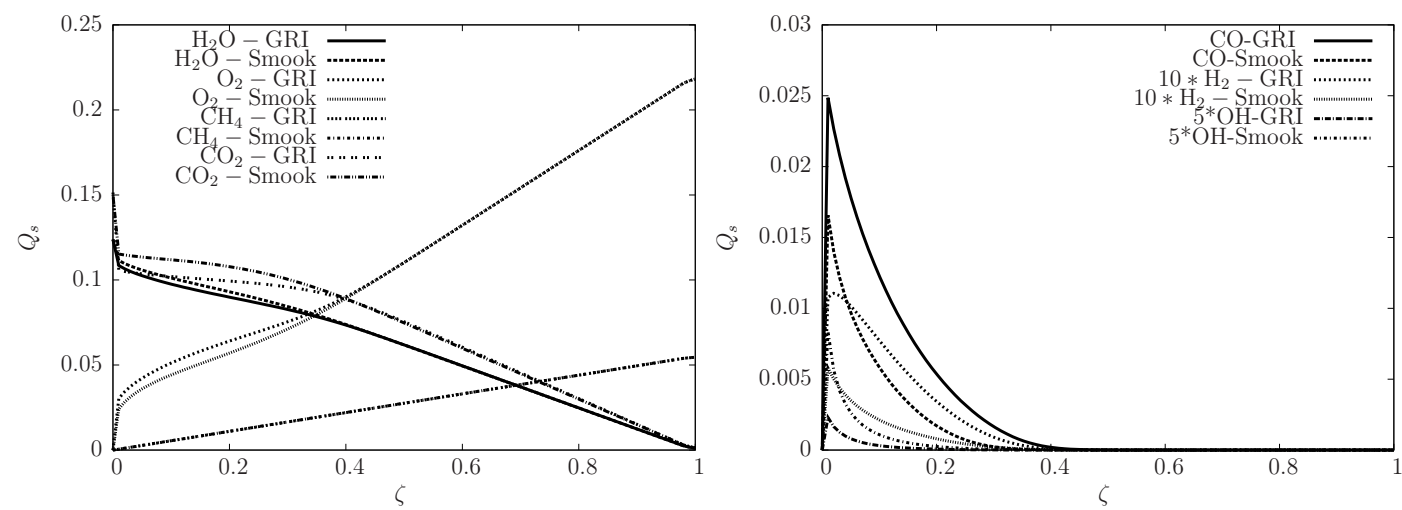

Figure 6.10: The variation of the conditional mean mass fraction with $\zeta$ for major and minor species in flame $\mathrm{F} 1$ at $\tilde{\theta}=0.55$ and $x / d=8.5$.

the numerical errors represented by the "imb." in Eq. (6.2) are observed to be very close to zero in Fig. 6.8 indicating that the equations are solved with small errors.

The typical variation of conditional mean mass fractions of representative major and minor species is shown in Figs. 6.9, 6.10 and 6.11 for flame F1. The results are shown for $\tilde{\theta}=0.55$, the middle of flame brush at $x / d=4.5,8.5$ and for the two kinetics mechanisms used. It is clear that the influence of the chemical kinetics mechanism on the computed values of the major species is 

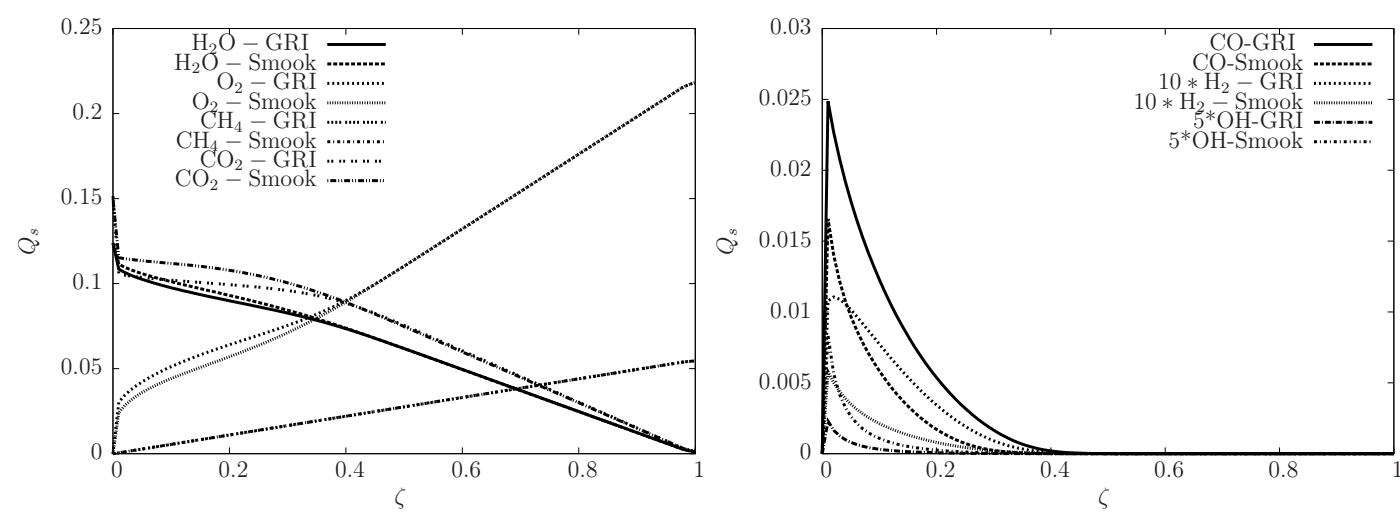

Figure 6.11: The variation of the conditional mean mass fraction with $\zeta$ for major and minor species in flame $\mathrm{F} 1$ at $\tilde{\theta}=0.55$ and $x / d=10.5$.

minimal. However, as one would expect the intermediate and the minor species values are strongly influenced by the kinetics mechanism. The results suggest that the skeletal mechanism gives lower values of $Q_{\alpha}$ compared to the GRI-mech. This is because of the additional chemical pathways available for the formation of the intermediate species in the GRI-mech. As seen from the calculated results that they have identical values at different axial locations $x / d=4.5,8.5$ and 10.5. This is supported by the CMC hypothesis that conditional mean quantities have weaker spatial dependence than unconditional quantities. Thus, CMC has a coarse grid compared to RANS, as indicated in section 6.1 .

\subsubsection{Comparison to Experimental Results}

To compute the mean values using Eq. (5.29), one requires the marginal PDF of the conditioning variable. As noted earlier, this PDF is obtained by presuming its shape as a Beta function, see Eq. (2.64), and using the computed values of $\tilde{\theta}$ and $\widetilde{\theta^{\prime \prime 2}}$. The PDF obtained thus in the CMC calculation is shown in Fig. 6.12 for flame F1. The typical bimodal shape, a well known characteristics of turbulent 


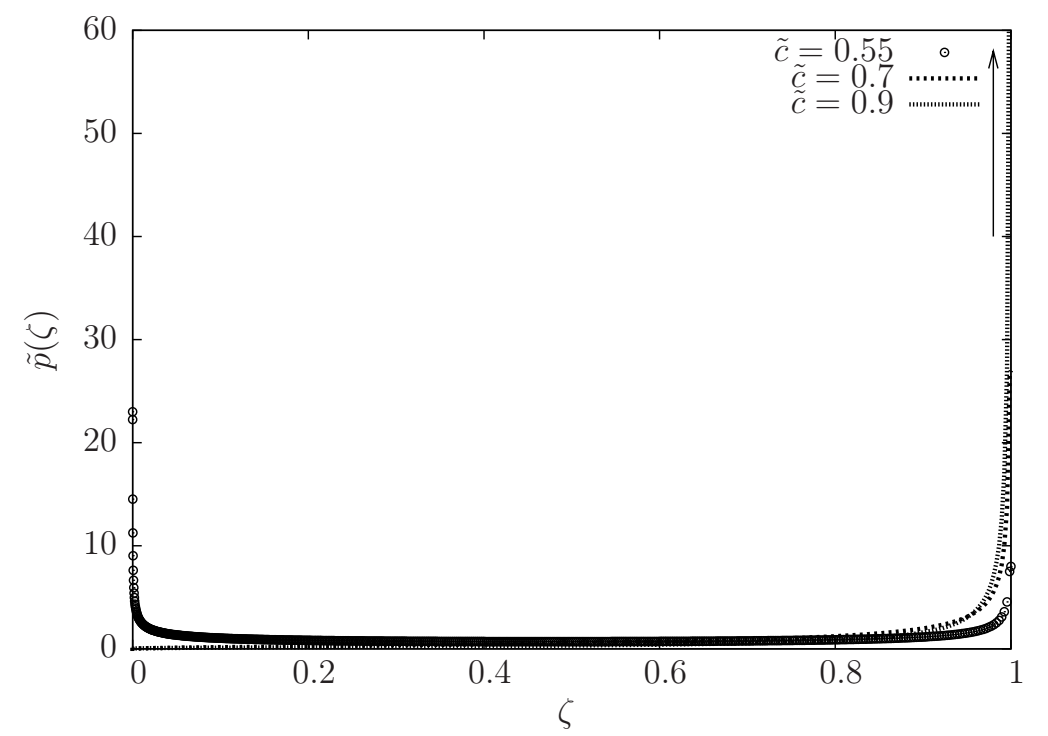

Figure 6.12: The variation of the Favre averaged PDF of the progress variable, $\tilde{p}(\zeta)$, at three locations in the flame brush of flame F1.

premixed flames, is seen in this figure for all locations shown.

Typical contours for the calculated Favre averaged $\tilde{\theta}, \widetilde{\theta^{\prime \prime 2}}, \tilde{Z}$ and $\tilde{T}$ are shown in Figs. 6.13 and 6.14 for flame F1 for visual inspection and the expect behaviour is seen clearly. The results from the reacting flow calculations are shown in Figs. 6.15 to 6.24 for up to five axial locations, the symbols are the experimental measurements and the lines are the computational results.

Figure 6.15 shows the radial variation of the normalised mean velocity and turbulent energy for five axial locations for flame F1. The results are shown for GRI-mech and Smooke's skeletal mechanism. The influence of $e_{Q_{\alpha}}$ is also shown. In general, the computed mean velocity agrees well with the measured values. The values computed using GRI-mech are closer to the experimental data, compared to the values obtained using Smooke's mechanism. The differential diffusion effects denoted by $e_{Q_{\alpha}}$ do not seem to influence the mean velocity values. However, the computed values of the turbulent kinetic energy show some 

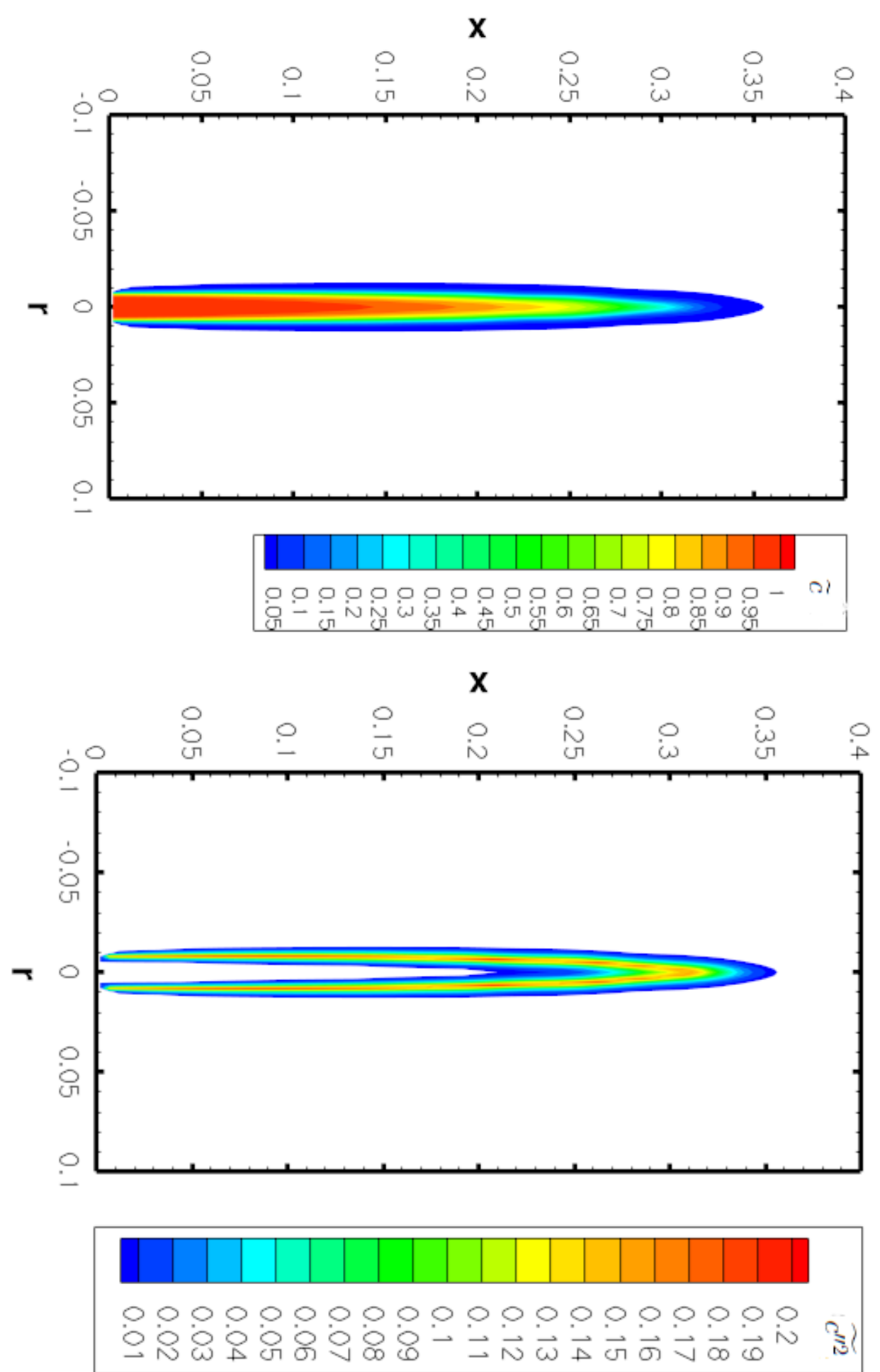

Figure 6.13: Contours of the mean progress variable and its variance for flame F1 

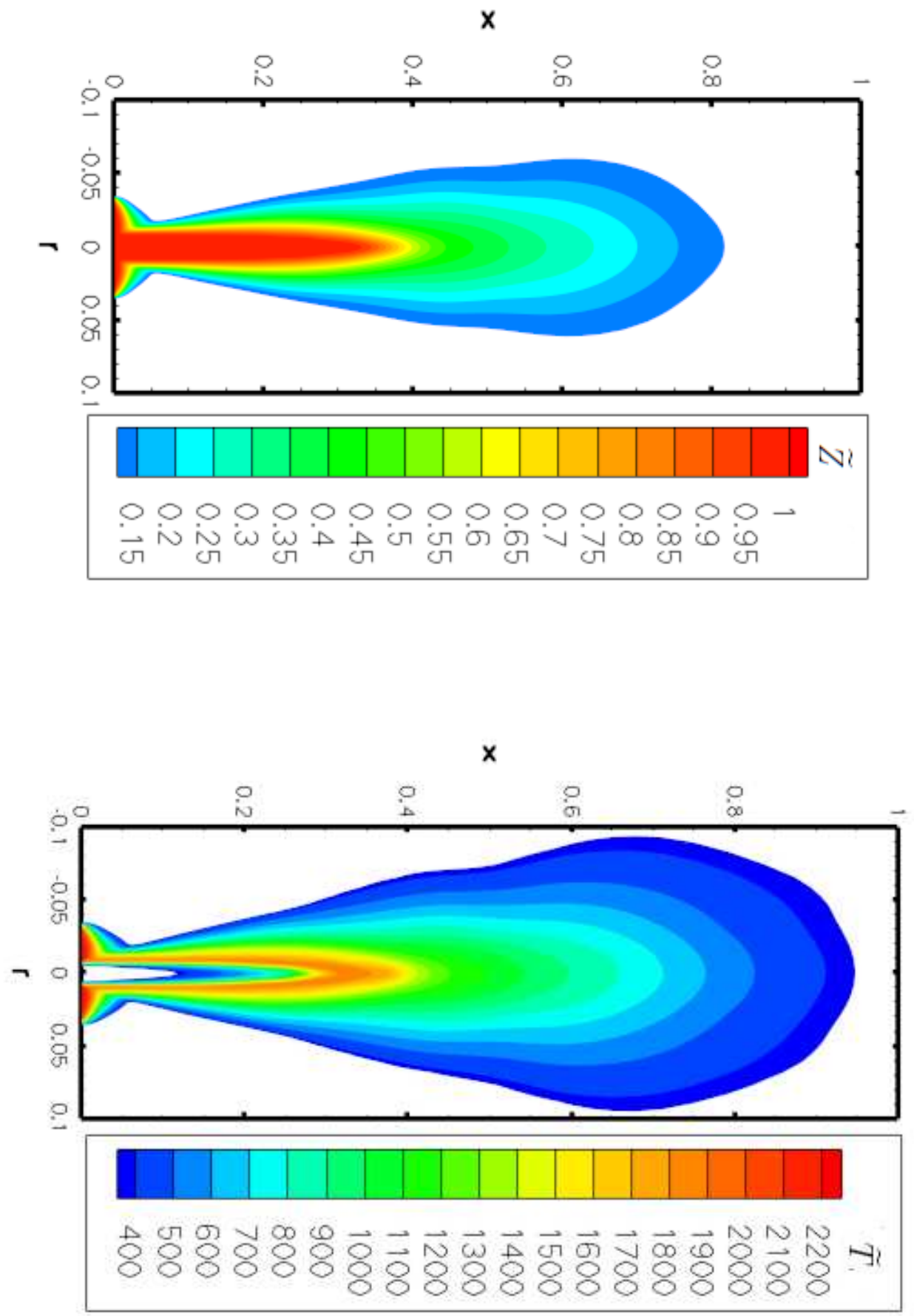

Figure 6.14: Contours of the mean mixture fraction variable and temperature for flame F1 


\section{Stoichiometric Premixed Flame Calculation}

sensitivity to the chemical kinetics mechanism and the differential diffusion effects. At $x / d=2.5$, the Smooke mechanism seem to work very well while the GRI-mech over predicts the turbulent kinetic energy. However, the contribution of $e_{Q_{\alpha}}$ seem to correct this over prediction. For all the axial locations studied in Fig 6.15, the turbulent kinetic energy computed using Smooke mechanism and GRI-mech with $e_{Q_{\alpha}}$ compares well with the measured values up to $r / d \simeq 0.5$ for flame F1. By comparing the results obtained using GRI-mech with and without $e_{Q_{\alpha}}$ contribution, one notes that the effects of $e_{Q_{\alpha}}$ is large up to $r / d=0.8$ in the near field and this region started to move slowly inward (toward the jet center) as $x / d$ increases. This suggests that the differential diffusion effects are important near the central core region. This is consistent with our expectation, since the turbulence is produced by the shear production mechanism and the turbulence in the jet is small.

The computed values of mean mass fraction of methane and normalised mean temperature are compared to the experimental data in Fig. 6.16 for flame F1. The agreement in the mean mass fraction of methane is very good and its sensitivity to the chemical kinetics mechanism is negligible. The mean temperature is over predicted in the near field and the agreement improves with down-stream distance. Except for $x / d=2.5$, the computed mean temperature is weakly sensitive to the chemical kinetics mechanism and differential diffusion effects. There is some uncertainty in the temperature boundary condition for the pilot stream as has been noted in many previous studies $[68 ; 79 ; 119 ; 120 ; 121]$ using this experiment and thus one must be careful while interpreting the results for the mean temperature at $x / d=2.5$. However, the general agreement between the computed and measured values is satisfactory and the computed values show negligibly small 


\section{Stoichiometric Premixed Flame Calculation}
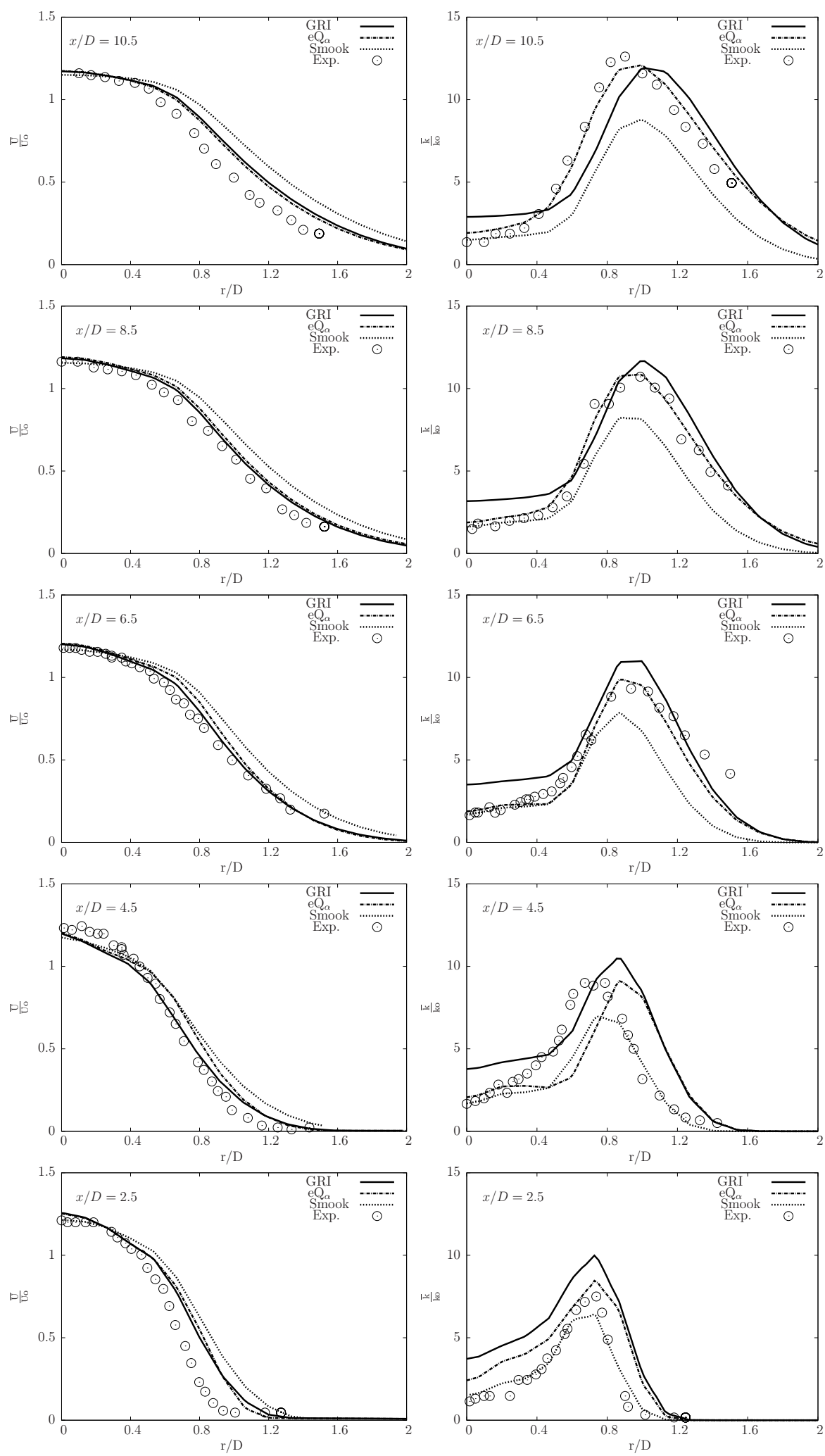

Figure 6.15: The normalised mean velocity and turbulent kinetic energy from the $\mathrm{CMC}$ calculations (lines) at five axial locations are compared to experimental results (symbols) for flame F1. 


\section{Stoichiometric Premixed Flame Calculation}

sensitivity to the chemical mechanism and the $e_{Q_{\alpha}}$ contribution. This observation also applies to the major species mass fraction reported in Fig. 6.17. The agreement for $\mathrm{H}_{2} \mathrm{O}$ and $\mathrm{O}_{2}$ is better than for $\mathrm{CO}_{2}$ as shown in Fig. 6.17 and these relative behaviours (between the computations and measurements) is similar to these reported in earlier studies using other combustion modelling methods.

The radial variation of mean mass fractions of $\mathrm{CO}, \mathrm{OH}$ and $\mathrm{H}_{2}$ are compared to the experimental measurements in Fig. 6.18 for five axial locations for flame F1. As one would expect the computed values are sensitive to the kinetics scheme, however, the influence of $e_{Q_{\alpha}}$ is observed to be small as in Fig. 6.18. The peak values of the averaged mass fraction of these species are larger for GRI mechanism compared to Smooke mechanism and the values obtained using Smooke mechanism are closer to the experimental data. The agreement between the computed and measured values are good for $\mathrm{OH}$ and $\mathrm{H}_{2}$ as shown in Fig. 6.18. However for $\mathrm{CO}$, there seems to be large discrepancies and the reason for this is unclear at this stage. The peak values and the radial variation of mean mass fraction of $\mathrm{CO}$ computed using $\mathrm{CMC}$ in this study are similar to those reported earlier using other modelling approaches for premixed flames.

The flames, F2 and F3, are simulated using the GRI-mechanism excluding the contributions from $e_{Q_{\alpha}}$. Figures 6.19 and 6.20 show the radial variation of the normalised mean velocity and turbulent kinetics energy for flames F2 and F3. The level of agreement between the computed and measured values of these quantities for flames F2 and F3 is similar to that shown for flame F1. At the center line, the computed mean velocity and turbulent energy are in excellent agreement with the experimental measurements for all axial locations for both flames F2 and F3. In the radial direction, the computed mean velocity agrees well with 


\section{Stoichiometric Premixed Flame Calculation}
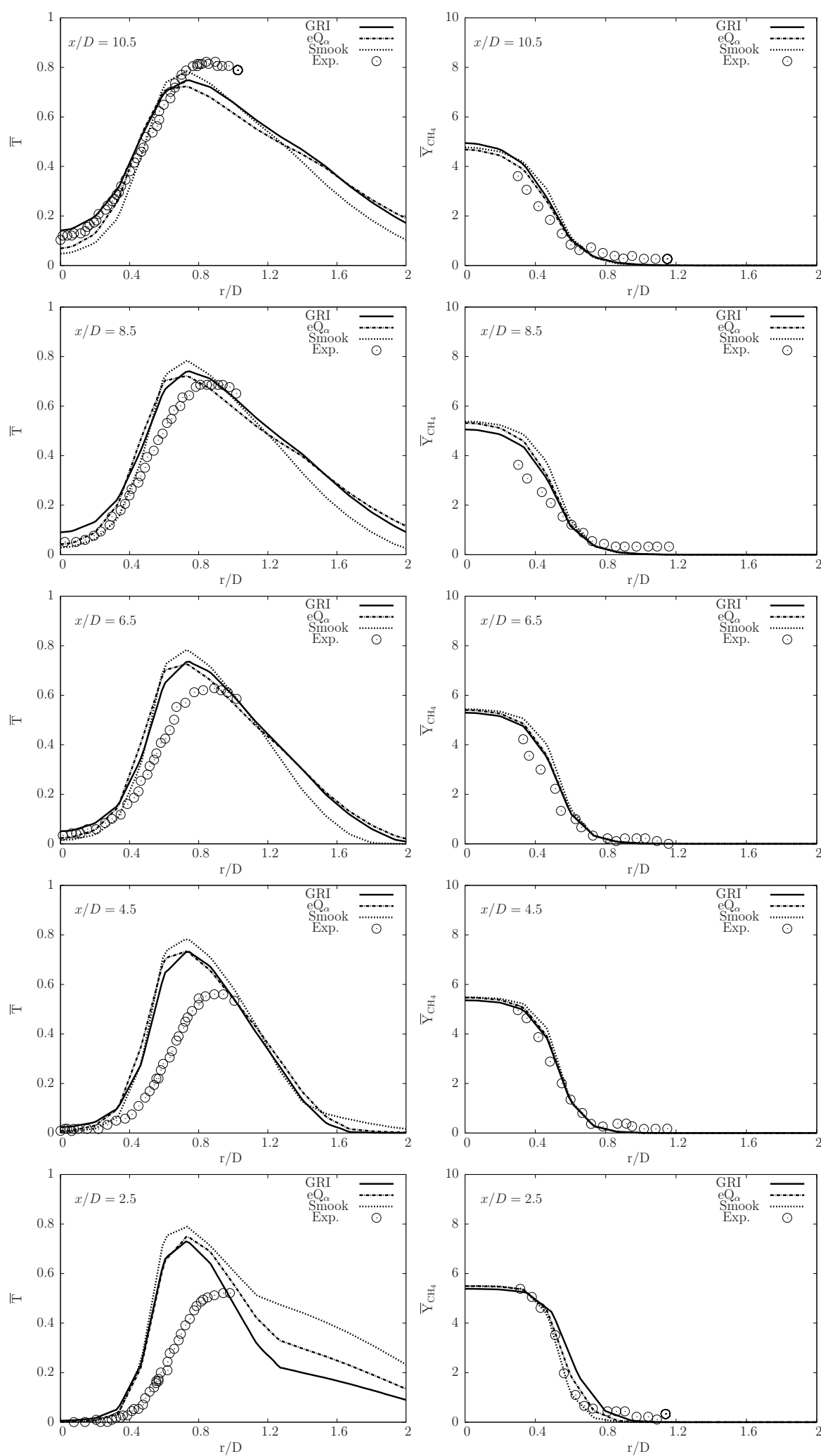

Figure 6.16: The normalised mean temperature and $\mathrm{CH}_{4}$ mass fraction $\times 100$, from the CMC calculations (lines) at five axial locations are compared to experimental results (symbols) for flame F1. 

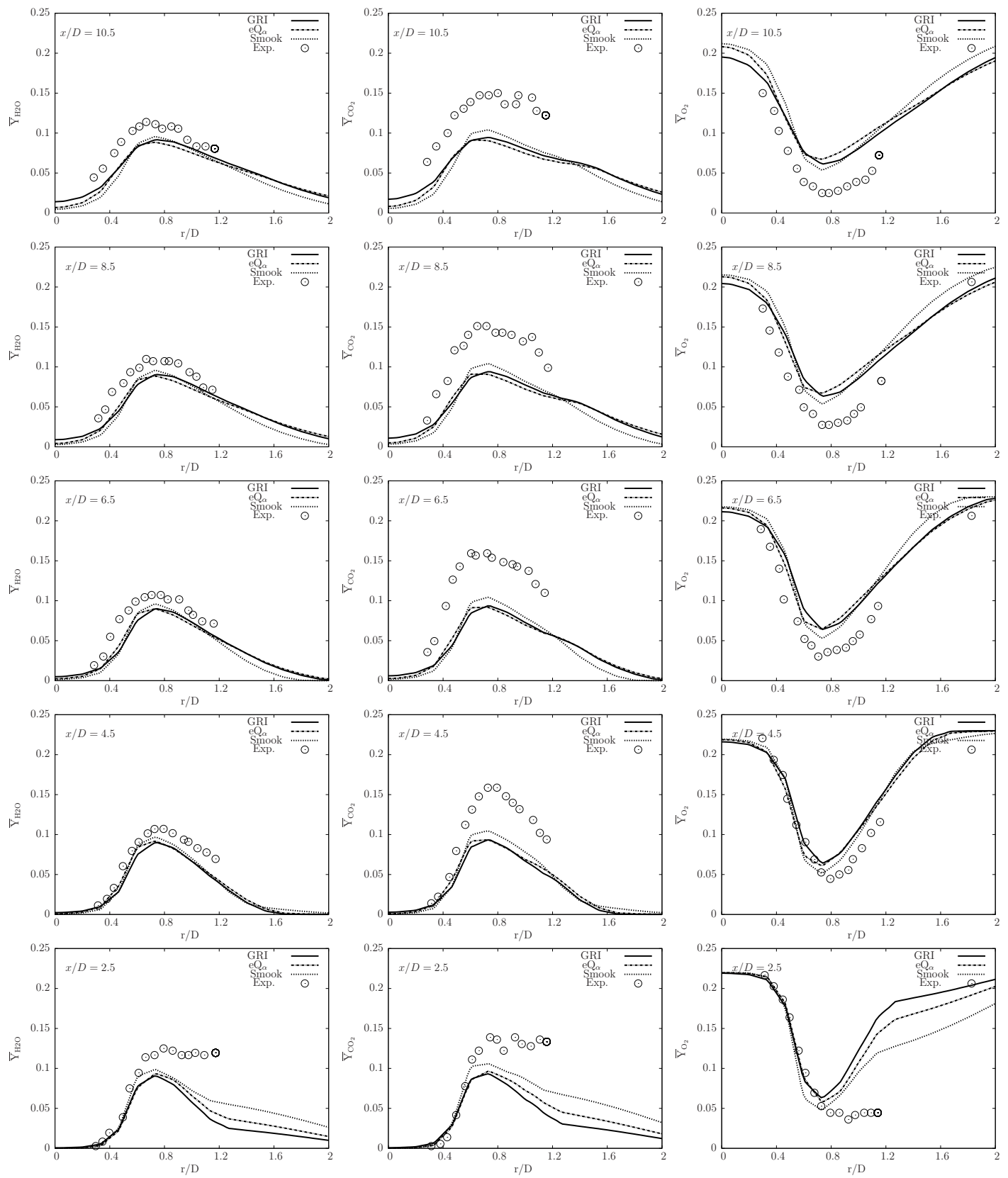

Figure 6.17: The mean mass fractions of $\mathrm{H}_{2} \mathrm{O}, \mathrm{CO}_{2}$ and $\mathrm{O}_{2}$ from the CMC calculations (lines) at five axial locations are compared to experimental results (symbols) for flame F1. 

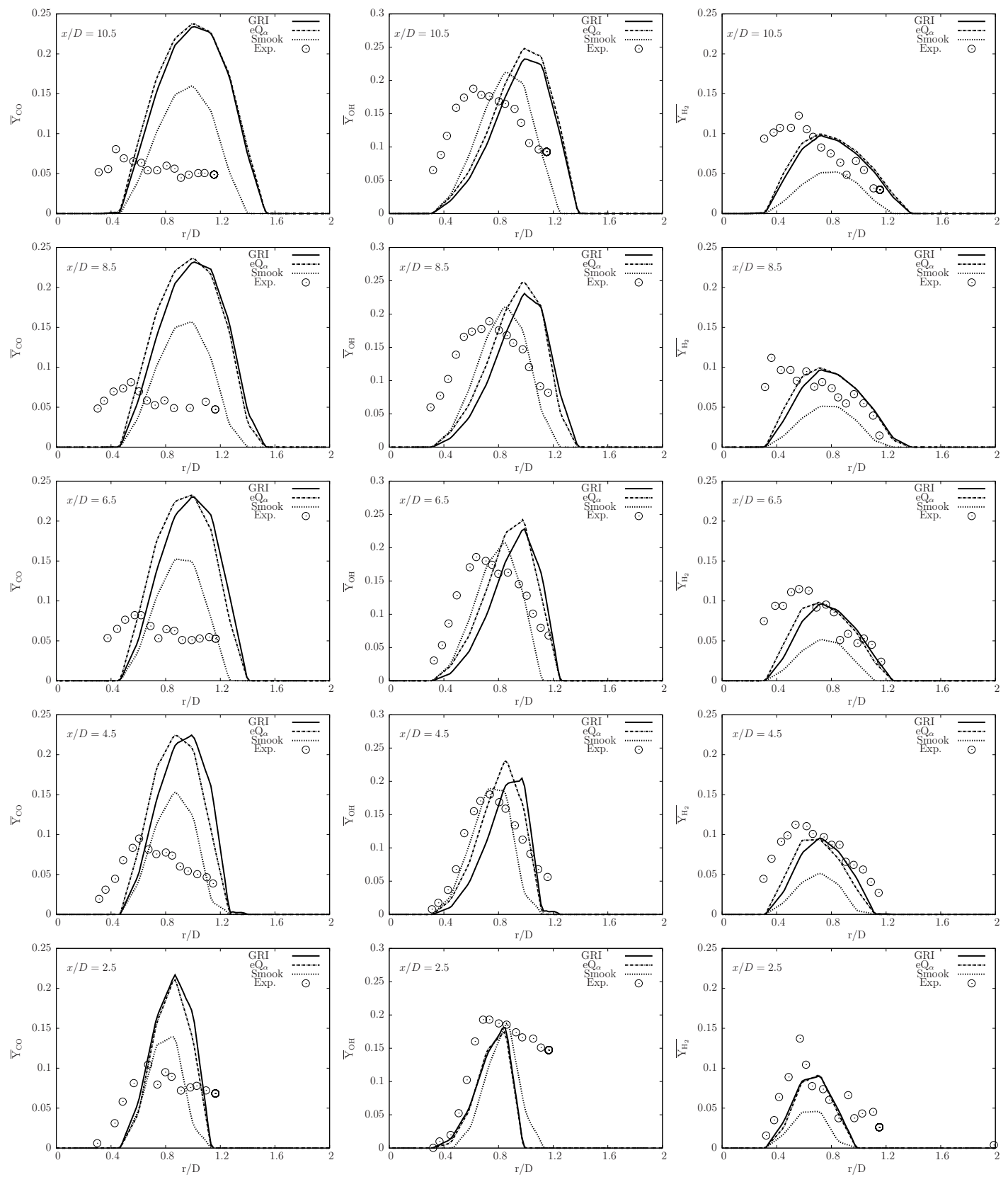

Figure 6.18: The mean mass fractions of $\mathrm{CO} \times 10, \mathrm{OH} \times 75$ and $\mathrm{H}_{2} \times 100$ from the $\mathrm{CMC}$ calculations (lines) at five axial locations are compared to experimental results (symbols) for flame F1. 


\section{Stoichiometric Premixed Flame Calculation}

the measurement values for flame F2, while flame F3 shows some discrepancies particularly at $r / d>0.8$. The turbulent energy is slightly over predicted in the near field and the agreement improves with down stream distance for flames F2 and F3.

The computed values of mean mass fraction of methane and normalised mean temperature of flames F2 and F3 are compared to the experimental data in Figs. 6.21 and 6.22. The level of agreement between the computed and measured values of the mean temperature and mass fraction of $\mathrm{CH}_{4}$ improves slightly as turbulence Reynolds number decreases (ie. from flame F1 to F3). As shown in the figure, the mean mass fraction of methane agrees well with experimental values and the mean temperature is over predicted in the near field and the agreement improves gradually in down stream direction. The computed values of the mean mass fraction of the major species of flames F2 and F3 are shown in Fig. 6.23. The major species are in good agreement in the near field and the agreement improves with down stream distance. The computed values of mean mass fractions of $\mathrm{CO}, \mathrm{OH}$ and $\mathrm{H}_{2}$ are compared to the experimental values in Fig. 6.24 for flames F2 and F3. The results are shown as radial variations at a few down stream locations. The relative behaviours between the calculated and measured values are similar to that for flame F1 for all species studied. The peak CO values measured for a given $x / d$ location seem to be weakly sensitive to the changes in the Reynolds and Damköhler numbers (compare the peak value, for example for $x / d=8.5$ among $\mathrm{F} 1, \mathrm{~F} 2$ and F3 flames), but the CMC results shows a gradual drop in the peak CO value as the Damköhler number increases. This is consistent with our expectation. 

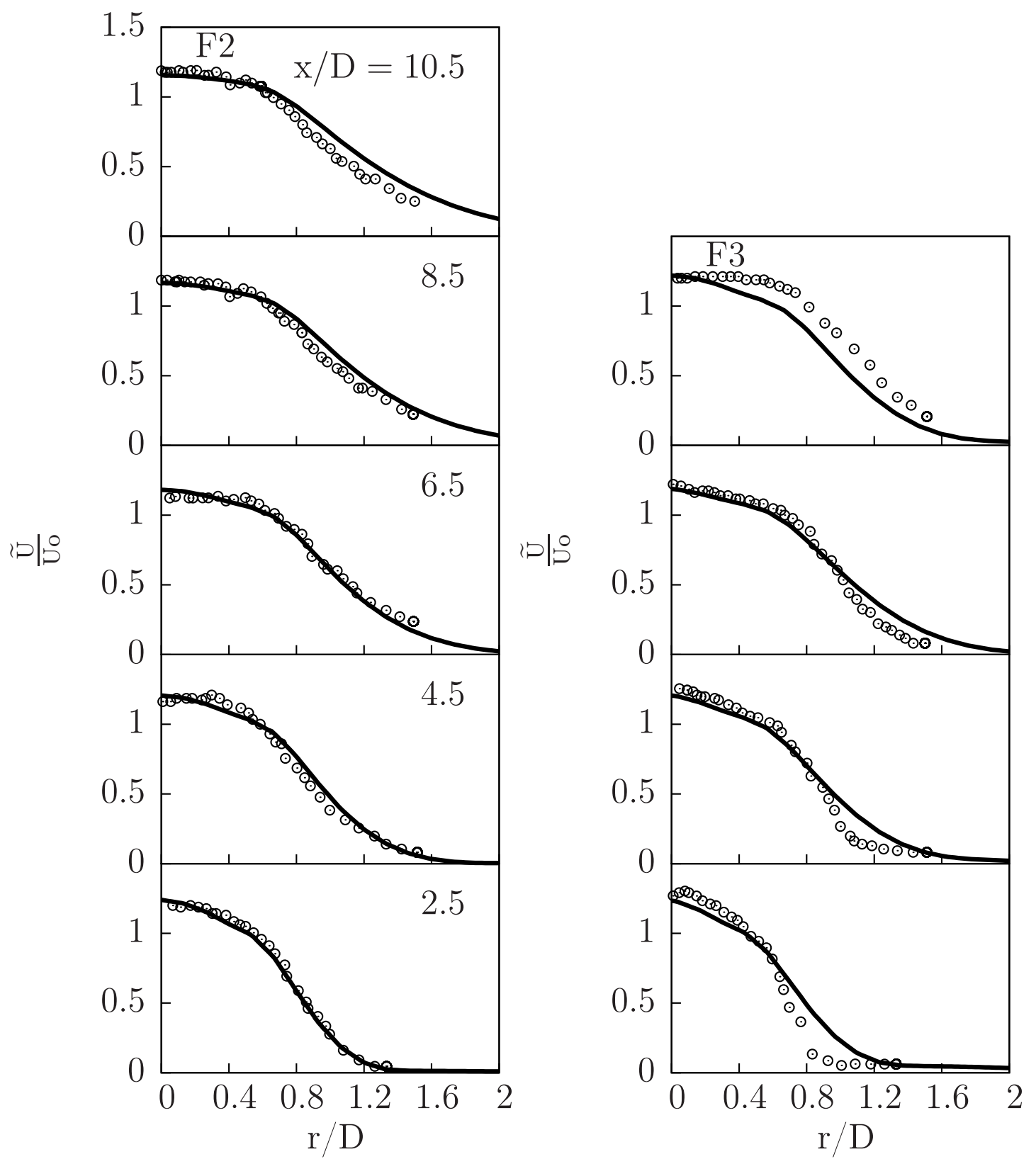

Figure 6.19: The normalised mean velocity from the CMC calculations (lines) at five axial locations are compared to experimental results (symbols) for flames F2 and F3. 

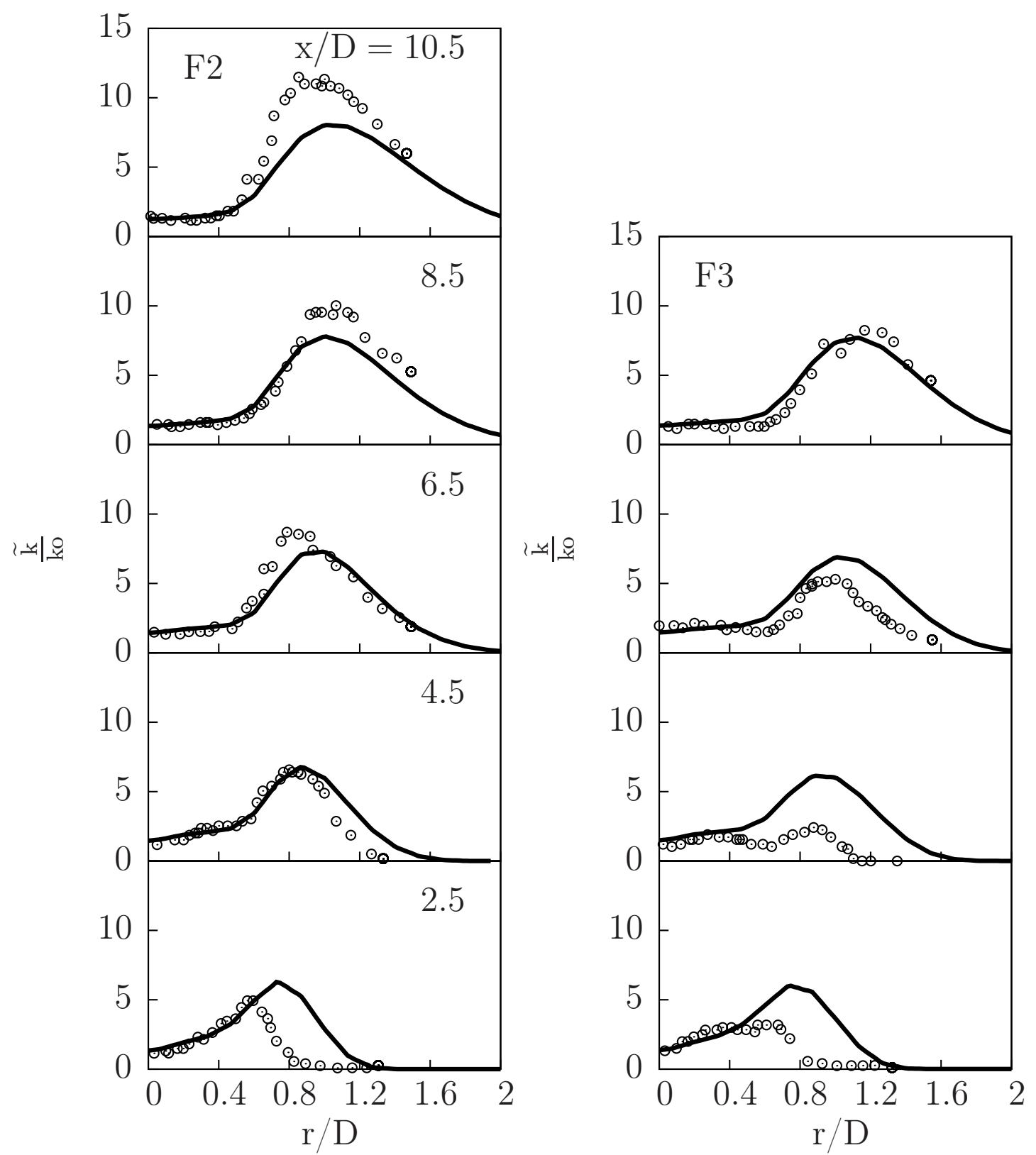

Figure 6.20: The normalised mean turbulent kinetic energy from the CMC calculations (lines) at five axial locations are compared to experimental results (symbols) for flames F2 and F3. 

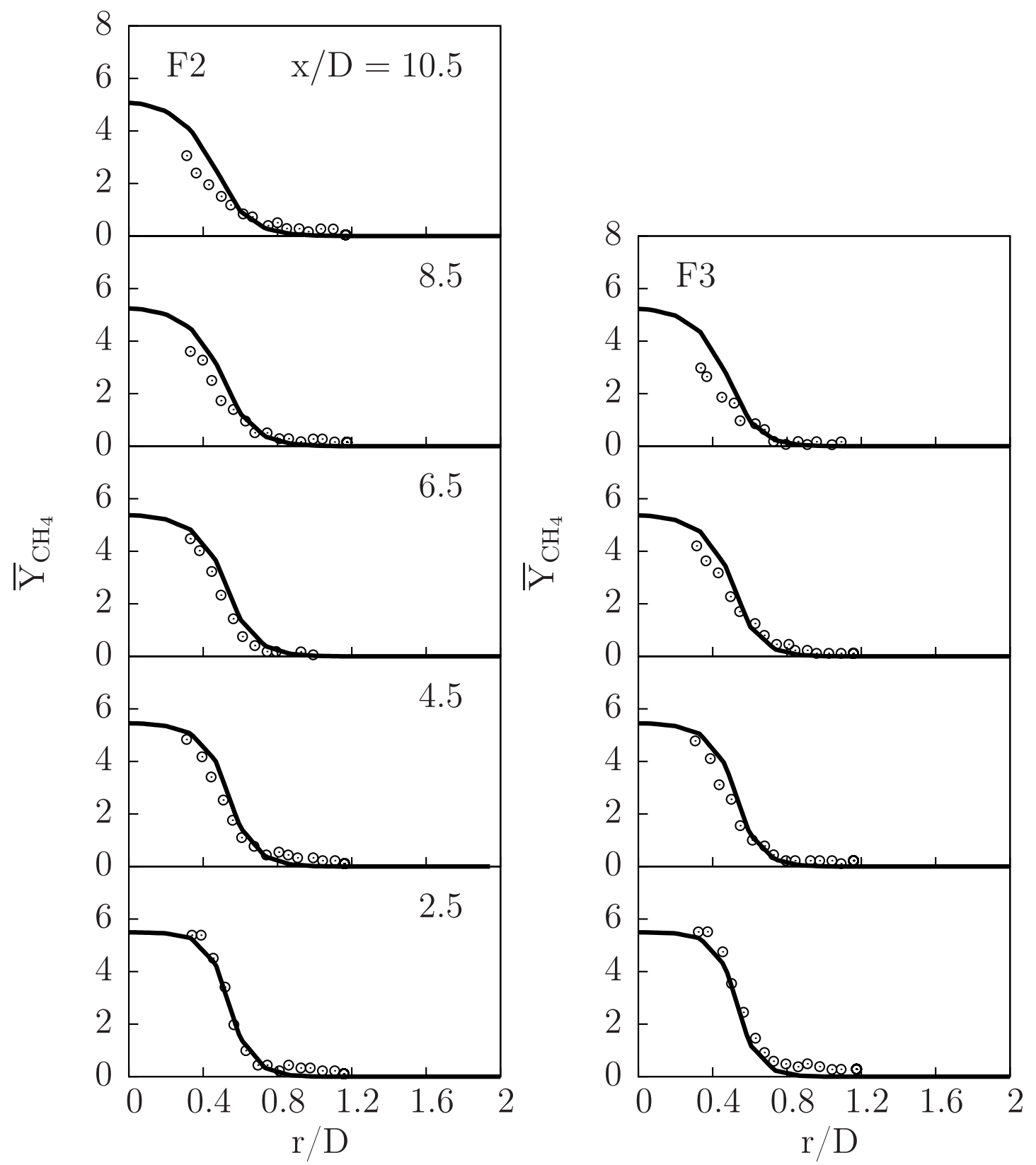

Figure 6.21: The normalised mean mass fraction of $\mathrm{CH}_{4} \times 100$ from the CMC calculations (lines) at five axial locations are compared to experimental results (symbols) for flames F2 and F3. 

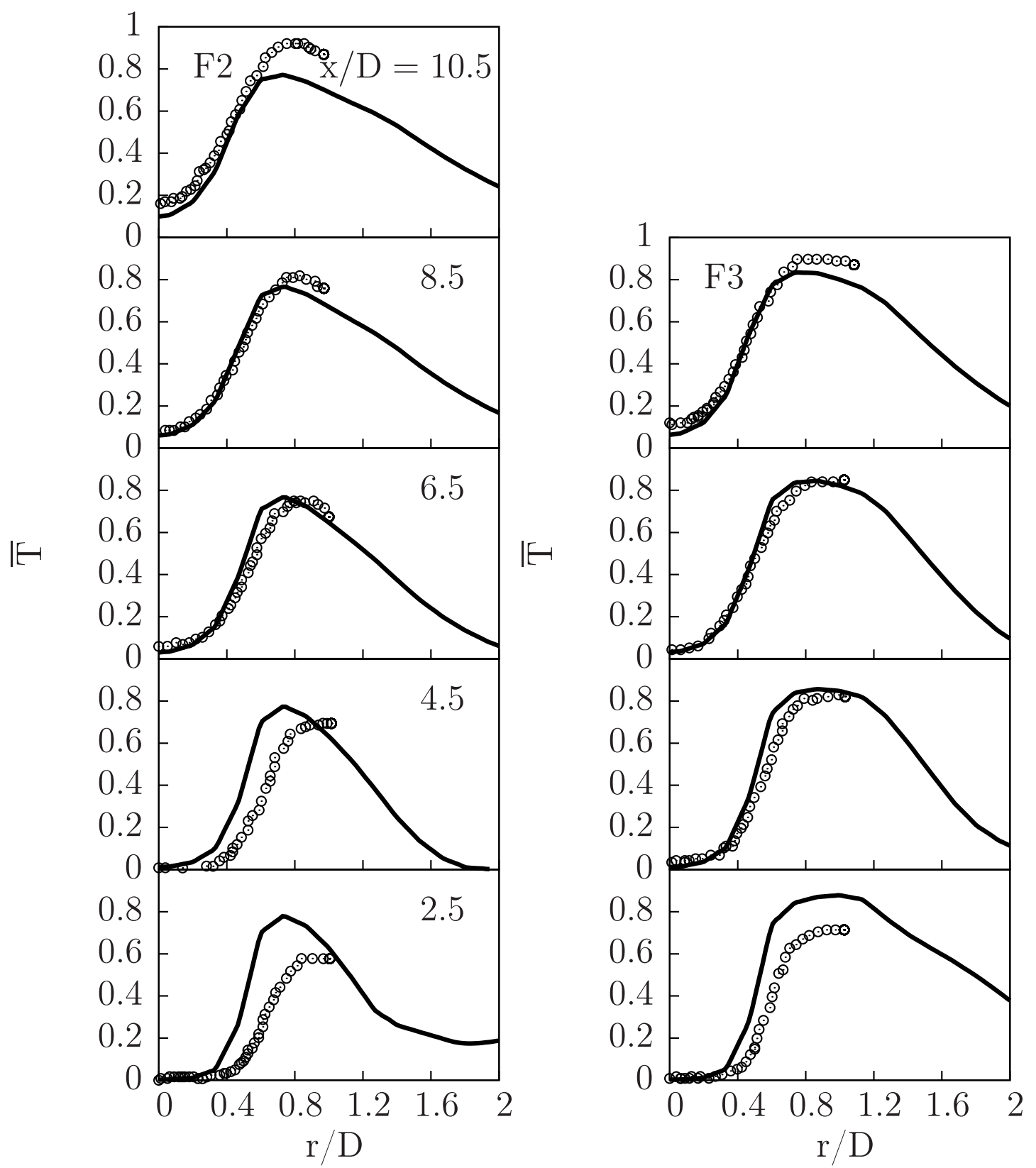

Figure 6.22: The normalised mean temperature from the CMC calculations (lines) at five axial locations are compared to experimental results (symbols) for flames F2 and F3. 

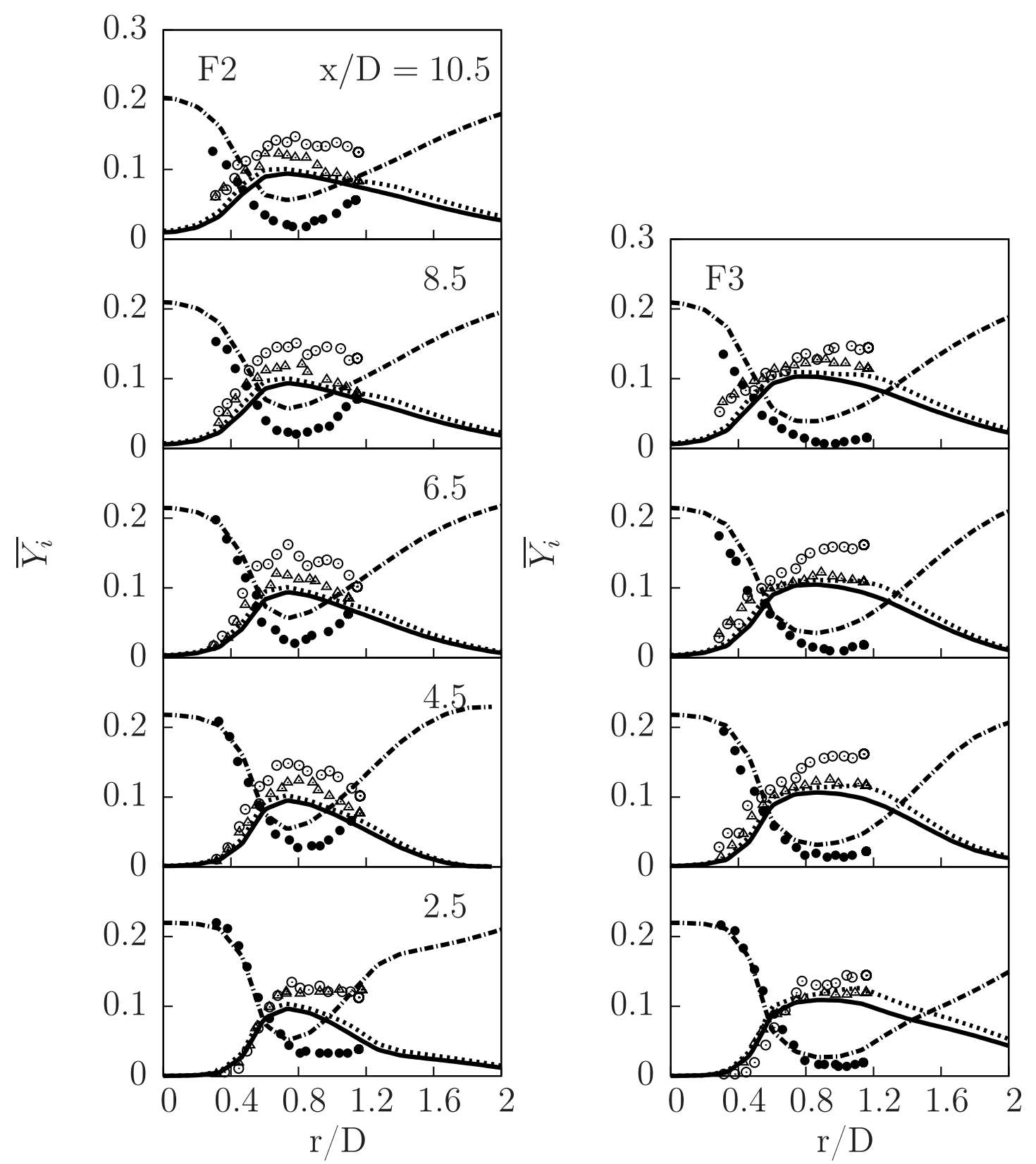

Figure 6.23: The mean mass fractions of $\mathrm{H}_{2} \mathrm{O}(\triangle,-), \mathrm{CO}_{2}(\circ, \cdots)$ and $\mathrm{O}_{2}(\bullet,-\cdot$ -) from the CMC calculations (lines) at five axial locations are compared to experimental results (symbols) for flames F2 and F3. 

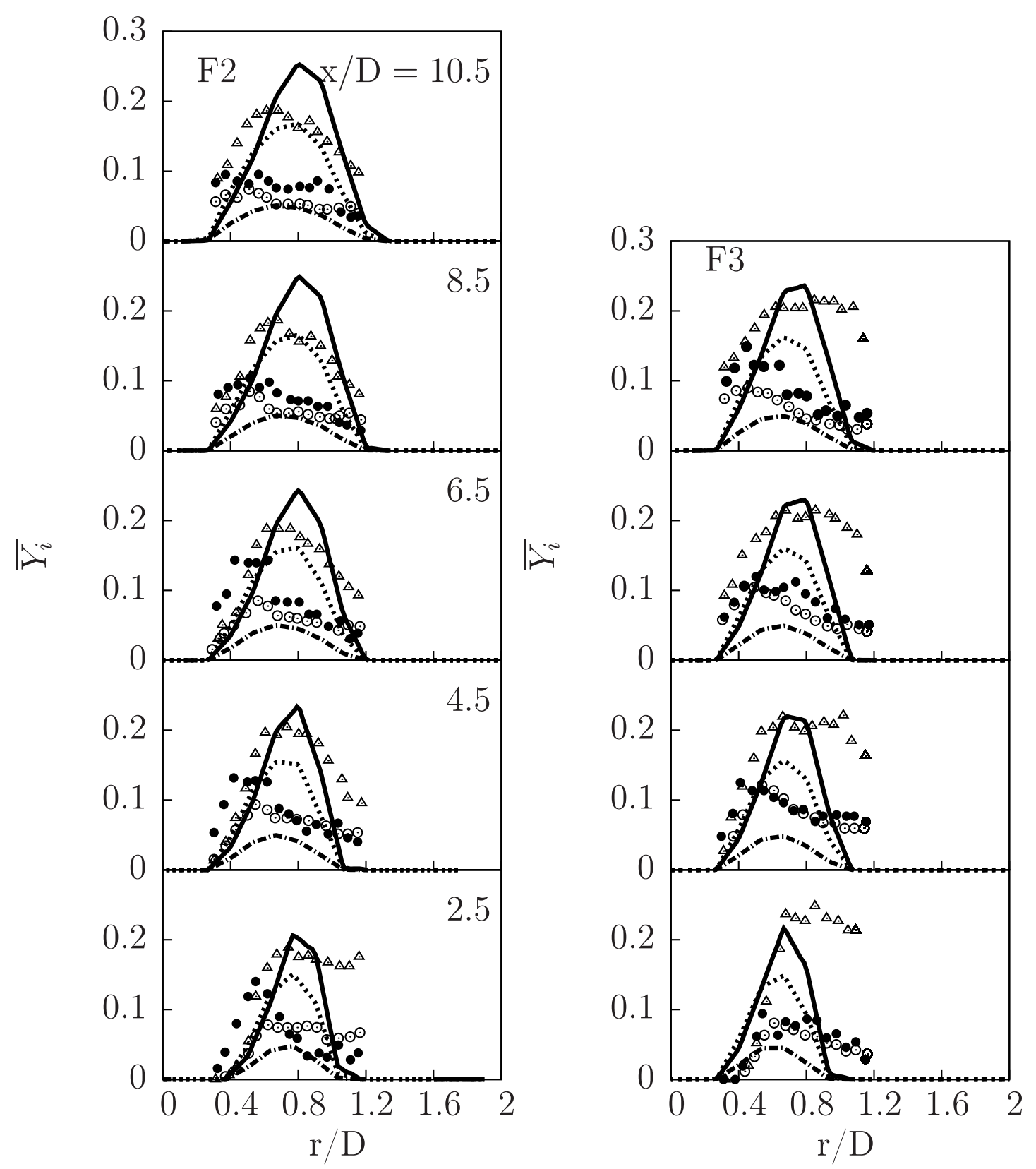

Figure 6.24: The mean mass fractions of $\mathrm{CO} \times 10(\circ, \cdots), \mathrm{OH} \times 75(\triangle,-)$ and $\mathrm{H}_{2} \times 100(\bullet,-\cdot-)$ from the CMC calculations (solid lines) at five axial locations are compared to experimental results (symbols) for flames F2 and F3. 


\section{Chapter 7}

\section{Lean Premixed Flame}

\section{Calculation}

\subsection{Flames Details}

The second test case used in this study is the pilot stabilised lean premixed flames of Dunn et al. [3], which is shown schematically in Fig. 7.1. This jet burner (PPJB) used in the experimental study [3] consists of a central jet of a lean premixed methane-air mixture with equivalence ratio of 0.5 and initial temperature of $300 \mathrm{~K}$, and a pilot of a hot stoichiometric premixed methaneair products. The central jet and the hot pilot have diameters of 4 and 23.5 $\mathrm{mm}$, respectively. These two streams are surrounded by a hot co-flow from lean premixed laminar $\mathrm{H}_{2}$-air flames with equivalence ratio of 0.43 . The diameter for this hot co-flow stream is $197 \mathrm{~mm}$. The pilot and the hot co-flow streams were flowing with small uniform velocities of 0.8 and $0.7 \mathrm{~m} / \mathrm{s}$, respectively [3]. Four flames were considered in the experimental study [3] designated as PM1- 


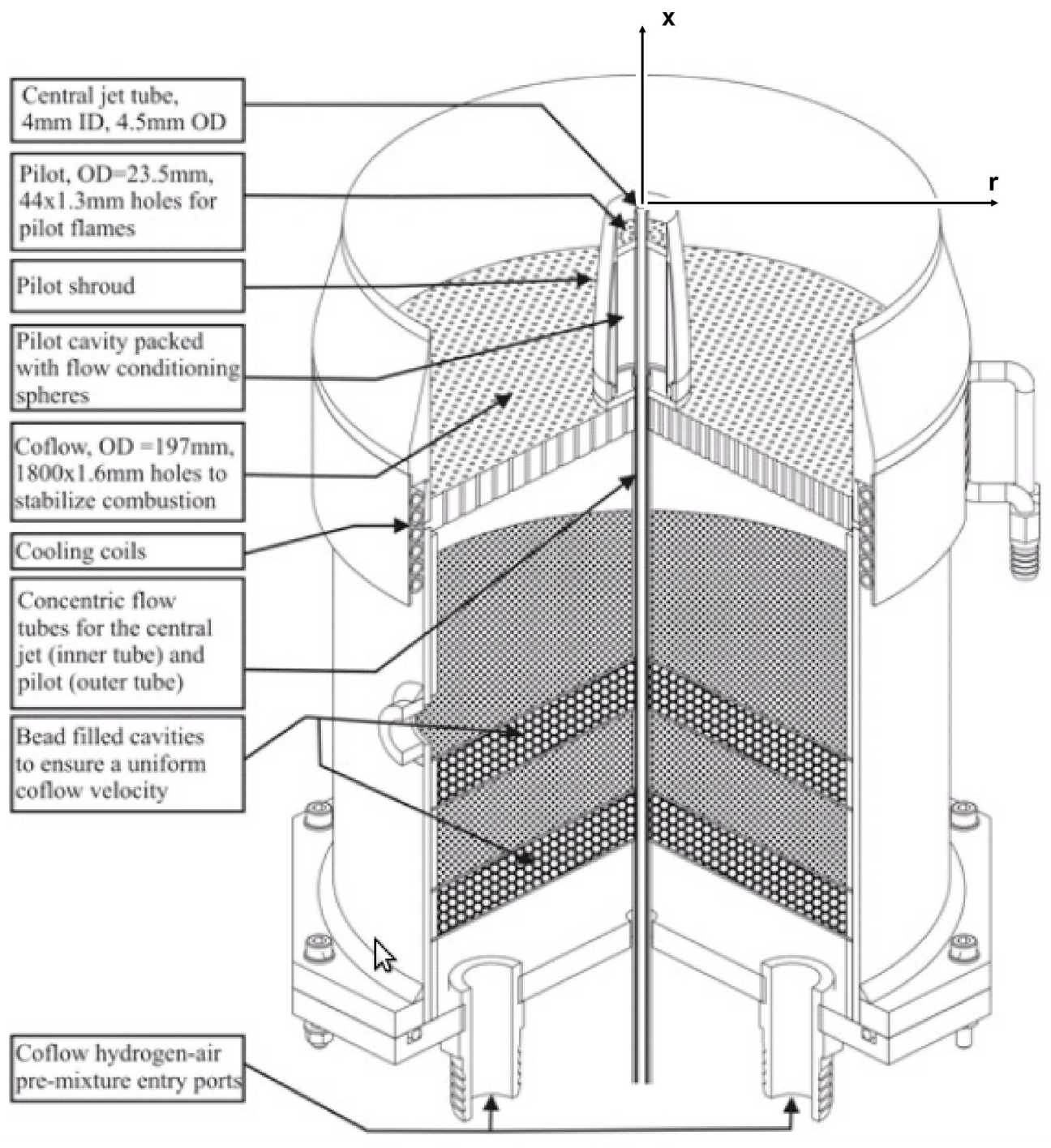

Figure 7.1: Schematic diagram of the premixed Bunsen burner from [3; 4]. 


\begin{tabular}{cccccc}
\hline \hline Stream & $D(\mathrm{~mm})$ & $U_{0}(\mathrm{~m} / \mathrm{s})$ & $T(\mathrm{~K})$ & $\phi$ & mixture \\
\hline Jet & 4 & $50-200$ & 300 & 0.5 & $\mathrm{CH}_{4}-$ air (reactant) \\
Pilot & 23.5 & 0.8 & 2280 & 1 & $\mathrm{CH}_{4}-$ air (products) \\
Co-flow & 197 & 0.7 & 1500 & 0.43 & $\mathrm{H}_{2}-$ air (products) \\
\hline
\end{tabular}

Table 7.1: The properties of the streams of flames PM1-50 and PM1-200.

50, PM1-100, PM1-150 and PM1-200 with central jet bulk mean velocities, $U_{o}$, of 50, 100, 150 and 200, respectively. The Reynolds number, Re, based on the central jet velocity, diameter, D, are 12500, 25000, 37000 and 50000, respectively. A small uniform velocity of $0.2 \mathrm{~m} / \mathrm{s}$ is specified to account for the ambient air entrainment. The characteristics of these streams are summarized in table 7.1.

As shown in the turbulent combustion regime diagram, Fig. 7.2, these flames are in the distributed combustion regime. In this regime, scales of the chemical length and time scales are larger than the turbulence. Hence, applying flamelets based methods to these flames may be inappropriate. Note that the values of $\Lambda / \delta$ for flames PM1-50 and PM1-200 are 2.4 and 1.8, respectively and the values of $u^{\prime}$ are 15 and 54, respectively as reported by Dunn et al.[125]. The laminar flame speed is $S_{L}^{o}=0.051 \mathrm{~m} / \mathrm{s}$. These flames have been used in earlier studies to validate premixed turbulent combustion models such as a transported PDF models [6] and joint velocity-turbulence frequency-composition VFJPDF method [5] and large eddy simulation [126]. In the current study, these flames are computed using the RANS-CMC approach discussed in earlier chapters of this thesis. 


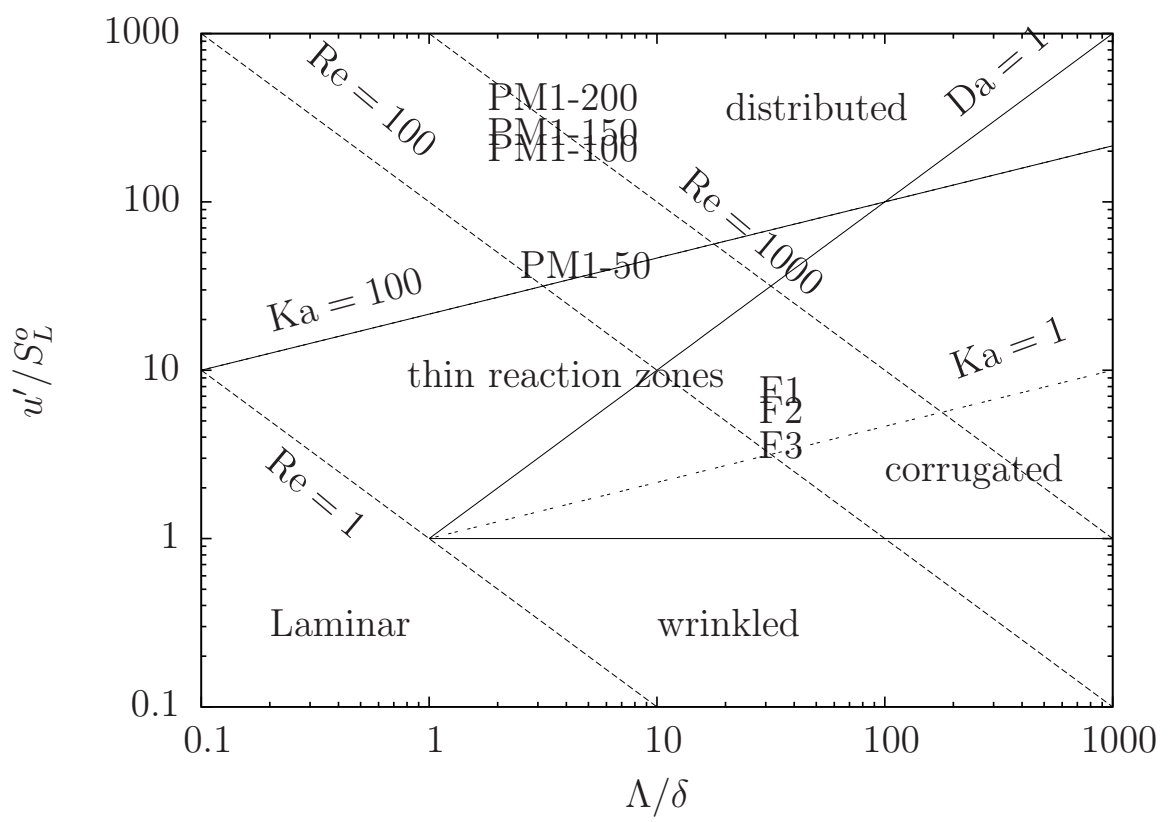

Figure 7.2: The premixed combustion regime diagram showing the stoichiometric and lean flames.

\subsection{Computational Details}

A schematic of the computational domain for this flame is shown in Fig 7.3. It must be noted that the three streams have different composition and equivalence ratios as given in table 7.1, and thus more than one passive scalar (fluid marker variable) is required to account for the mixing of these streams. Thus, in addition to the Favre averaged transport equations used for the stoichiometric premixed flames discussed in chapter 6 , additional transport equations for two passive scalars, $\widetilde{Z_{2}}$ and $\widetilde{Z_{3}}$, are implemented to track the fluids emerging from the hot pilot and hot co-flow streams and to account for the effects of mixing of these streams with the main jet and with the entrained air. Thus, each stream has its own designated markers, $\widetilde{Z_{1}}, \widetilde{Z_{2}}$ and $\widetilde{Z_{3}}$ and their values are set to be unity at the inlet of appropriate streams as shown in Fig 7.3. These markers are used in 
the following manner to obtain the average temperature $\widetilde{T}$. The total enthalpy computed using its transport equation at a given grid point in the physical space is written as

$$
\begin{aligned}
& \widetilde{h}=h^{s}+\Delta h_{f, \operatorname{mix}}^{o} \\
& =\sum_{i}\left[\widetilde{Z_{1}} \widetilde{Y_{\alpha}}+\widetilde{Z_{2}} \widetilde{Y_{\alpha, 2}}+\widetilde{Z_{3}} \widetilde{Y_{\alpha, 3}}+\left(1-\widetilde{Z_{1}}-\widetilde{Z_{2}}-\widetilde{Z_{3}}\right) \widetilde{Y_{\alpha, a i r}}\right] \theta_{p_{i}}\left(\widetilde{T}-T_{0}\right) \\
& +\sum_{i}\left(\widetilde{Z_{1}} \widetilde{Y_{\alpha}}+\widetilde{Z_{2}} \widetilde{Y_{\alpha, 2}}+\widetilde{Z_{3}} \widetilde{Y_{\alpha, 3}}\right) \Delta h_{f_{\alpha}}^{o}
\end{aligned}
$$

where $\widetilde{Y_{\alpha}}$ is the mass fraction of species $\alpha$ at the grid point. $\widetilde{Y_{\alpha, 2}}$ and $\widetilde{Y_{\alpha, 3}}$ are the mass fractions of species $\alpha$ in the streams 2 and 3 at the inlet. $\Delta h_{f_{\alpha}}^{o}$ is the enthalpy of formation for species $\alpha$ and the enthalpy of formation for air is taken to be zero. From the above equation, one can easily verify that the temperature of the incoming streams are recovered by using the boundary values for $\widetilde{Z_{1}}, \widetilde{Z_{2}}$ and $\widetilde{Z_{3}}$ marked in Fig 7.3.

The inlet velocity profile is specified using the bulk mean value as $u(r) / U_{0}=$ $(1-r / R)^{1 / 7}$, where $R$ is the radius of the main jet port. Uniform velocities of 0.8 and $0.7 \mathrm{~m} / \mathrm{s}$ are specified as initial values for the pilot and hot co-flow streams. A small uniform velocity of $0.2 \mathrm{~m} / \mathrm{s}$ is specified for the surroundings air. A uniform value for the mean turbulent kinetic energy, $\widetilde{k}_{o}$, at the center line of the fuel nozzle exit, is obtained from $I=u^{\prime} / U_{0}=\sqrt{2 k / 3} / U_{0}$. The axial rms velocity, $u^{\prime}$, for the cold and reacting flows for both selected flames are reported in the experiment [3], see $u^{\prime}$, Figs. 7.4 and 7.5. The initial value for dissipation rate, $\widetilde{\epsilon}_{o}$, 


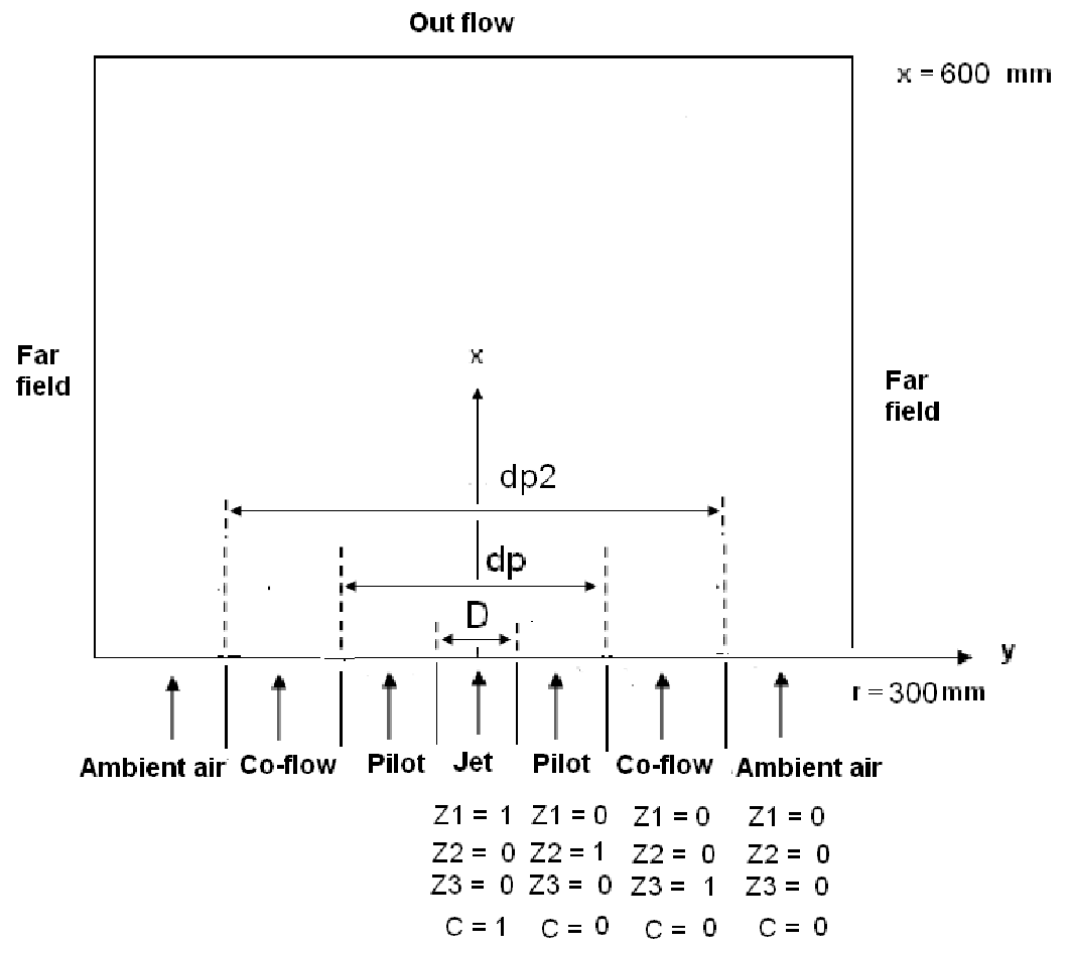

Figure 7.3: Schematic diagram of the burner setup and the computational domain along with BCs. 
at the center line of the fuel nozzle exit, is obtained using $\widetilde{\epsilon}_{o}=C_{u} \widetilde{k}_{o}^{3 / 2} / \Lambda$.

The computational domain is $600 \mathrm{~mm}$ long in the axial direction, $x$, and $300 \mathrm{~mm}$ in the radial direction, $r$. The CFD computational domain has 150 cells in the axial direction and 100 cells in the radial direction. The domain is refined non uniformly near the fuel, pilot and co-flow streams, where the smallest cell size is $1 \times 1.6$ (in mm). As for the stoichiometric flame, the CMC grid has 500 non-uniform cells in $\zeta$ space, which were required to resolve the strong gradients in the progress variable space. Two cells in each direction of the physical space grid for the RANS equations are combined to create the physical space grid for the CMC equations. The sensitivity of the computed solution to the grid size is tested by doubling and halving the size of the smallest cell, while retaining the grow the rate for the cell size. The solution showed a weak sensitivity and thus, the grid resolution noted above is used for the results reported in this chapter.

The values of various model parameters in the turbulence and other models discussed in earlier chapters are kept to be the same as those used for stoichiometric flame studied in chapter 6 , except for $C_{\epsilon 1}=1.6$. The chemical kinetics is modelled using the GRI-mechanism [123] and a steady flamelet solution is used as an initial solution and to specify the boundary conditions for the CMC equations. The computed solutions are compared to the experimental data reported in $[4 ; 6]$ in the following discussion.

\subsection{Results and Discussion}

Turbulence models and boundary conditions used in this calculation are assessed first by comparing the non-reacting flow results for both flames, PM1-200 and 

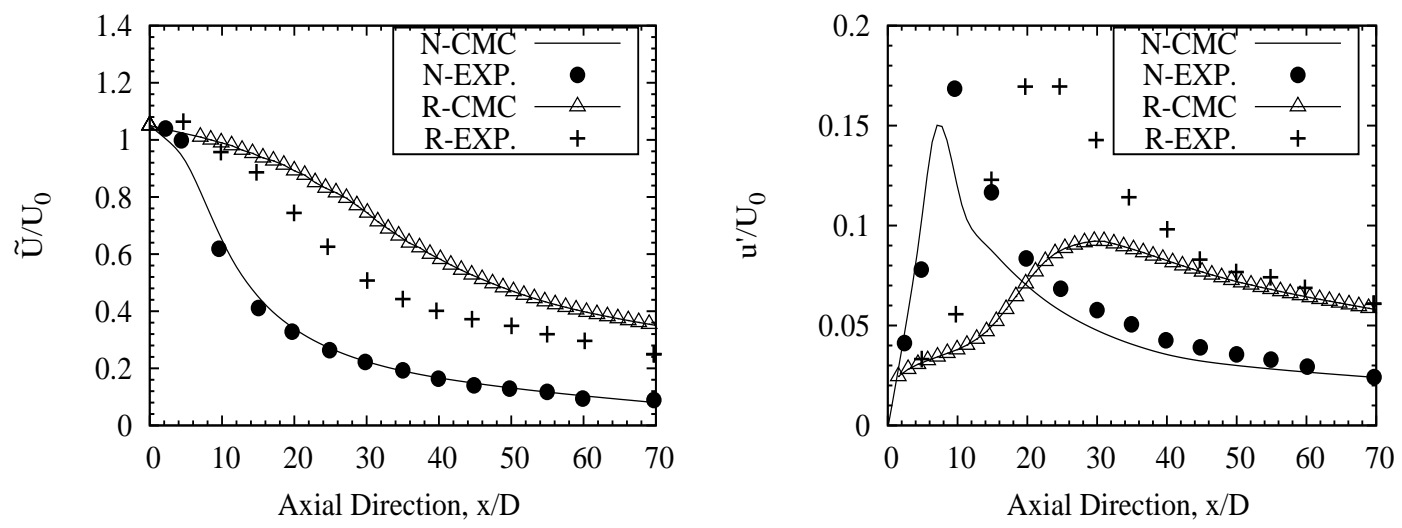

Figure 7.4: The calculated (lines) normalised mean axial velocity and rms velocity from non-reacting and reacting flows are compared to experimental data (symbols) for flame PM1-200.

PM1-50 selected for this study. Figures. 7.4 and 7.5 compares the calculated mean and rms axial velocity. The values are normalised using the bulk mean velocity, $U_{0}$, at the jet exit. The non-reacting and reacting flows are denoted by the letter $\mathrm{N}$ and $\mathrm{R}$, respectively in these figures. The normalised mean axial velocity for both of these flames are in excellent agreement with the measurements. A very good agreement for the normalized rms velocity is observed. These comparison clearly indicated that the boundary conditions used in the simulations mimic the experimental conditions well. Note that the $u^{\prime}$ is obtained from the calculated $\widetilde{k}$ as explained in the previous section, while the experimental results is obtained using

$$
u^{\prime}=\sqrt{\frac{\sum_{i=1}^{i=n}\left[u\left(t_{i}\right)-\bar{U}\right]^{2} \Delta t_{i}}{\sum_{i=1}^{i=n} \Delta t_{i}}},
$$

where $n$ is taken to be the number of realisations and $\Delta t_{i}$ is the gate period in the measurements for the $i^{\text {th }}$ realisation of the velocity. 

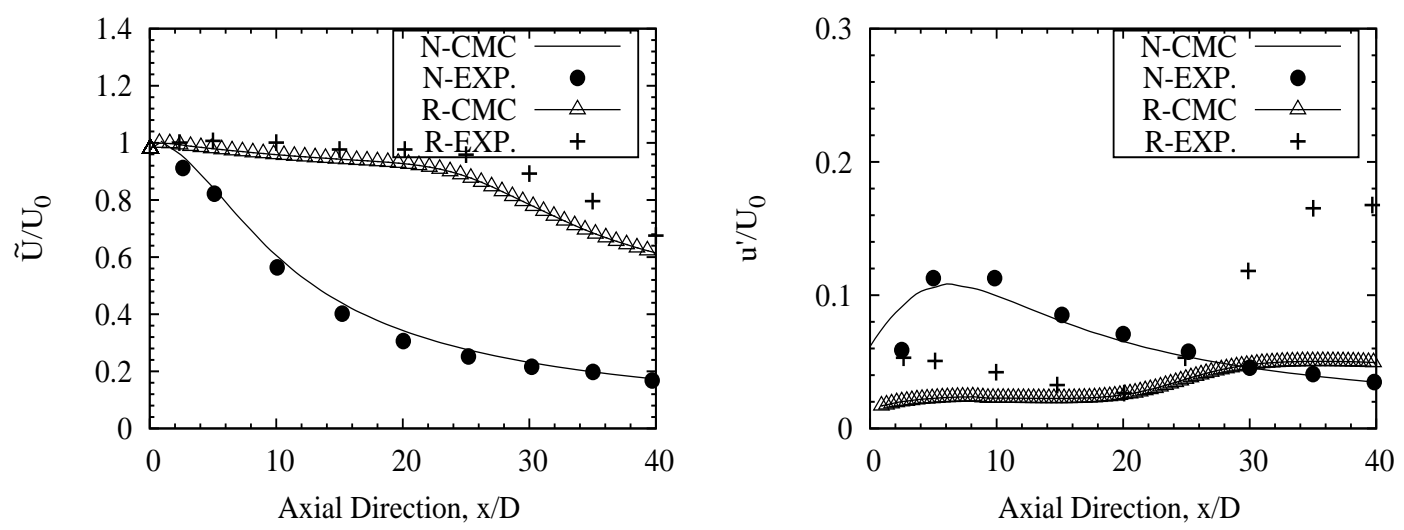

Figure 7.5: The calculated (lines) normalised mean axial velocity and rms velocity from non-reacting and reacting flows are compared to experimental data (symbols) for flame PM1-50.

\subsubsection{Conditional Mean Mass Fractions}

The conditional mean mass fraction, $Q_{\alpha}$, is obtained by solving the CMC equation, Eq. (3.3). The typical variation of conditional mean mass fractions for some selected representative major and minor species is shown in Figs. 7.6, 7.7 and 7.8 for flame PM1-200. The results are shown for $\tilde{\theta}=0.55$, the middle of flame brush at axial location $x / D=4.5,8.5$ and 10.5. $\zeta=0$ is the product side and $\zeta=1$ is the reactant side. Note that, the values of $\mathrm{H}_{2}$ and $\mathrm{OH}$ are multiplied by 40 and 20, respectively for plotting purpose. The unstrained laminar premixed flame is computed using CHEMKIN-II package along with GRI-mech. The variation of conditional averages of major species in the sample space is very close to those found in the unstrained laminar flame. However, the representative minor species variation with $\zeta$ in the $\mathrm{CMC}$ calculation is similar to those observed in the laminar flame, except for the molecular hydrogen. In the laminar flame, the hydrogen mass fraction varies little with a sharp drop near $\zeta=0$ and $\zeta=1$, but 

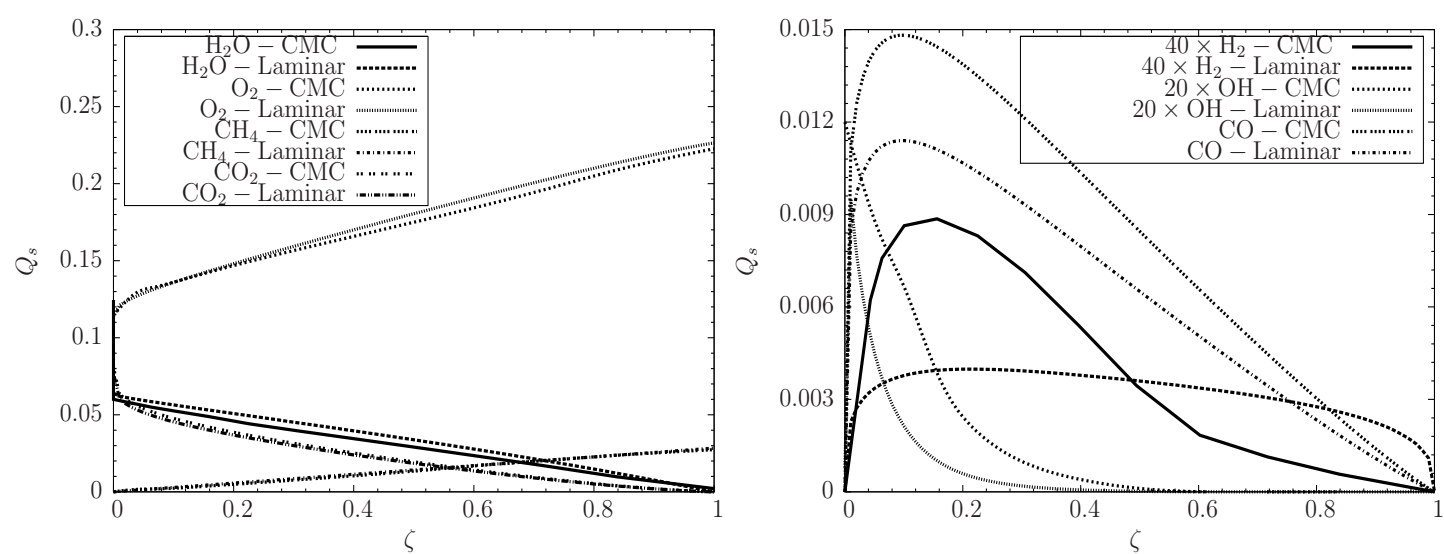

Figure 7.6: The variation of the conditional mean mass fraction with $\zeta$ for major and minor species for flame PM1-200 at $\tilde{\theta}=0.55$ and $x / D=4.5$.

the conditional mean value for the hydrogen shows a peak near $\zeta \approx 0.16$. For the PM1-50 flame, the variation of conditional means are similar to those shown in Fig. 7.6. As seen from the calculated results that they have identical values at different axial locations $x / d=4.5,8.5$ and 10.5. This is supported by the CMC hypothesis that conditional mean quantities have weaker spatial dependence than unconditional quantities.

\subsubsection{Comparison to Experimental Results}

The typical variation of the conditional dissipation rate for the flames, PM1200 and PM1-50 is shown in Figs. 7.9, 7.10 and 7.11 at $\tilde{\theta}=0.55$ and axial locations $x / D=4.5,6.5$ and 10.5. The turbulence level for flame PM1-200 is higher compared to flame PM1-50, and thus the mean dissipation rates are larger in flame PM1-200. The variation of $\left\langle N_{\theta} \mid \zeta\right\rangle^{+}$shown in Figs $7.9,7.10$ and 7.11 are similar to that observed in Fig 6.6 for the stoichiometric flame and the magnitudes are different because of the difference in the thermo-chemical 

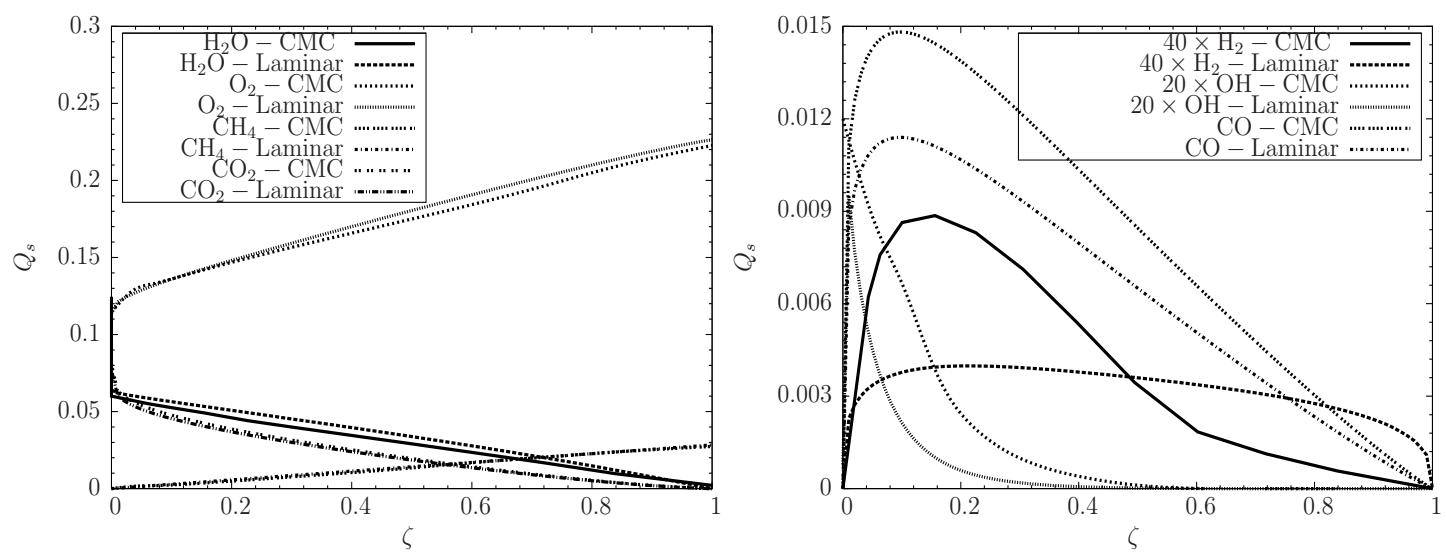

Figure 7.7: The variation of the conditional mean mass fraction with $\zeta$ for major and minor species for flame PM1-200 at $\tilde{\theta}=0.55$ and $x / D=8.5$.
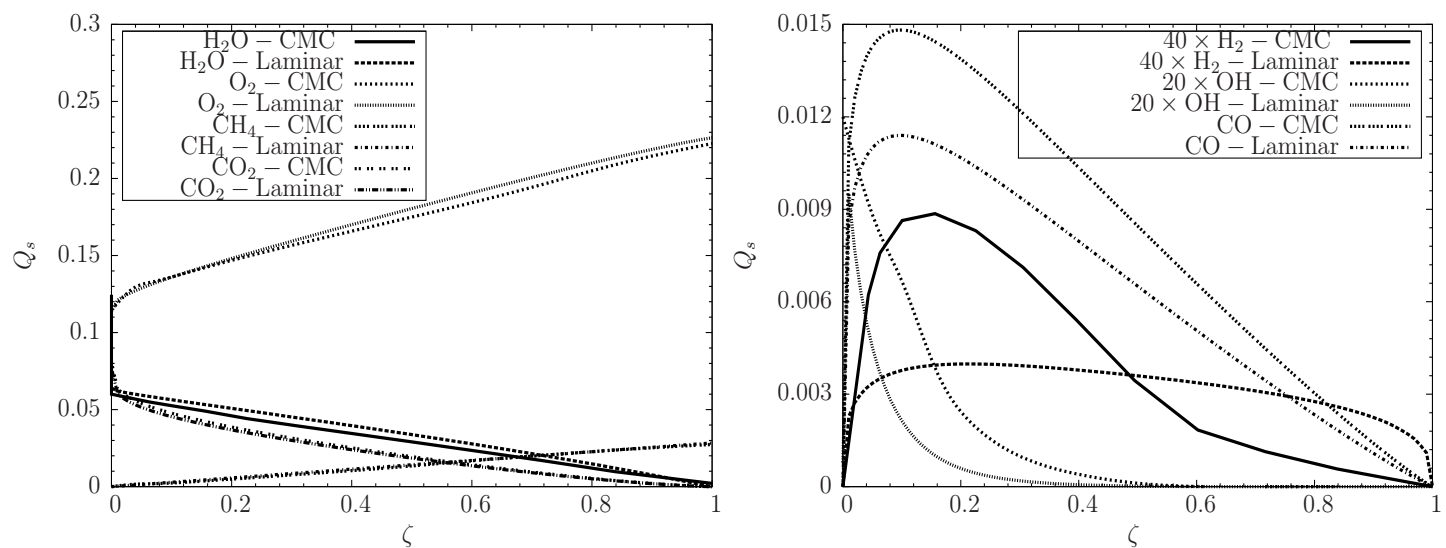

Figure 7.8: The variation of the conditional mean mass fraction with $\zeta$ for major and minor species for flame PM1-200 at $\tilde{\theta}=0.55$ and $x / D=10.5$. 


\begin{tabular}{ccccc}
\hline \hline$\widetilde{\theta}$ & $\widetilde{\theta^{\prime \prime 2}}$ & $\widetilde{Z_{1}}$ & $\widetilde{Z_{2}}$ & $\widetilde{Z_{3}}$ \\
\hline 0.9 & 0.027 & 0.971 & 0.026 & 0.0025 \\
0.8 & 0.063 & 0.931 & 0.061 & 0.006 \\
0.7 & 0.097 & 0.894 & 0.094 & 0.011 \\
0.6 & 0.129 & 0.857 & 0.126 & 0.015 \\
0.5 & 0.161 & 0.821 & 0.158 & 0.020 \\
0.4 & 0.193 & 0.784 & 0.190 & 0.024 \\
0.2 & 0.145 & 0.635 & 0.308 & 0.055 \\
\hline
\end{tabular}

Table 7.2: Values of $\widetilde{\theta}, \widetilde{\theta^{\prime \prime 2}}, \widetilde{Z_{1}}, \widetilde{Z_{2}}$ and $\widetilde{Z_{3}}$ in flame PM1-50 for the PDFs shown in Fig. 7.12.

and turbulence conditions of these flames. Also it obvious from the results that the conditional scalar dissipation rate changes slowly in the axial direction. Its magnitude is increased about $5 \%$ in the down stream direction.

The marginal PDF of the progress variable, obtained using the presumed Beta function for the given Favre mean and variance is shown in Figs. 7.12 and 7.13 for flames PM1-50 and PM1-200, respectively. The Favre mean, $\widetilde{\theta}$, and variance, $\widetilde{\theta^{\prime \prime 2}}$, are obtained from their respective transport equations. Unlike for the stoichiometric flames discussed in chapter 6, these PDFs are not bimodal across the flame brush. These PDFs are shown for seven different radial locations, denoted by $\widetilde{\theta}$, at a given axial location $x / D=4.5$. The values of $\widetilde{\theta^{\prime \prime 2}}, \widetilde{Z_{1}}, \widetilde{Z_{2}}$ and $\widetilde{Z}_{3}$ at these locations are given in tables 7.2 and 7.3 respectively for flames PM150 and PM1-200. The value of the Favre variance for the bimodal PDF limit given by $\widetilde{\theta^{\prime \prime 2}}=\widetilde{\theta}(1-\widetilde{\theta})$ is observed only for low values of $\widetilde{\theta}$. At location very close to the burnt side of the flame brush, ie. $\widetilde{\theta} \leq 0.15$, bimodal PDFs are observed for both flames PM1-50 and PM1-200. The mono-modal behaviour of this marginal PDF is typical of distributed combustion regime.

The radial variations of $\widetilde{Z_{1}}, \widetilde{Z}_{2}$ and $\widetilde{Z_{3}}$ at six axial locations in flame PM1-200 


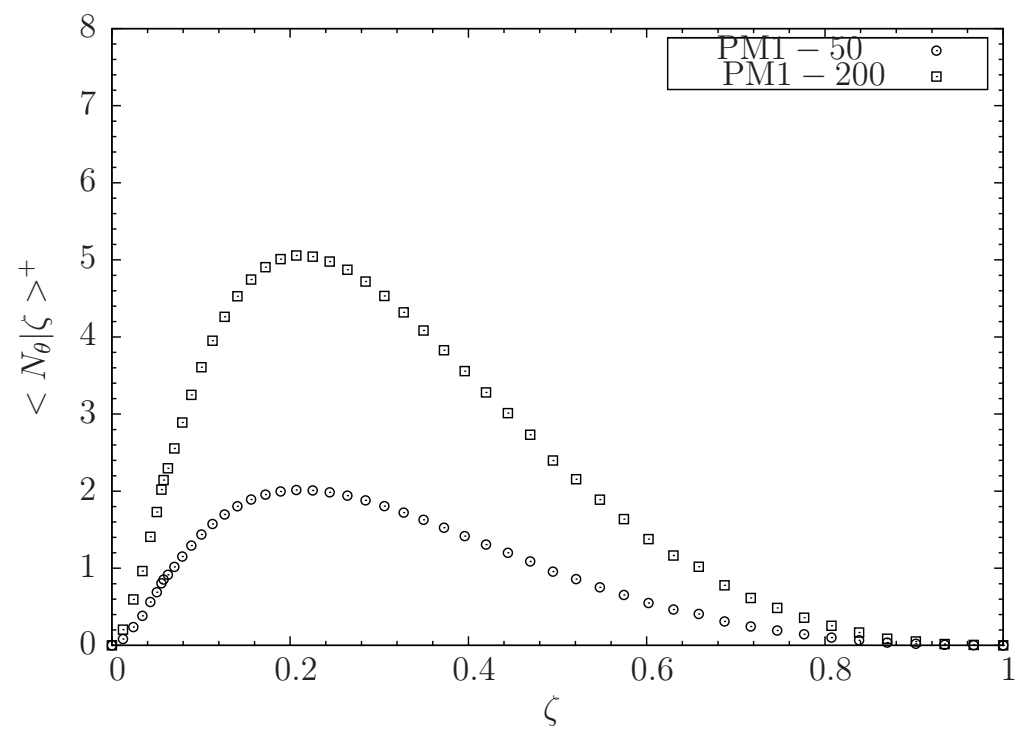

Figure 7.9: The typical variation of the conditional scalar dissipation rate, $\left\langle N_{\theta}^{+} \mid \zeta\right\rangle$, obtained in the CMC calculation for flames PM1-50 and PM1-200 at $\tilde{\theta}=0.55$, $x / D=4.5$. The values are non-dimensionalised using $S_{L}^{o}$ and $\delta_{L}^{o}$.

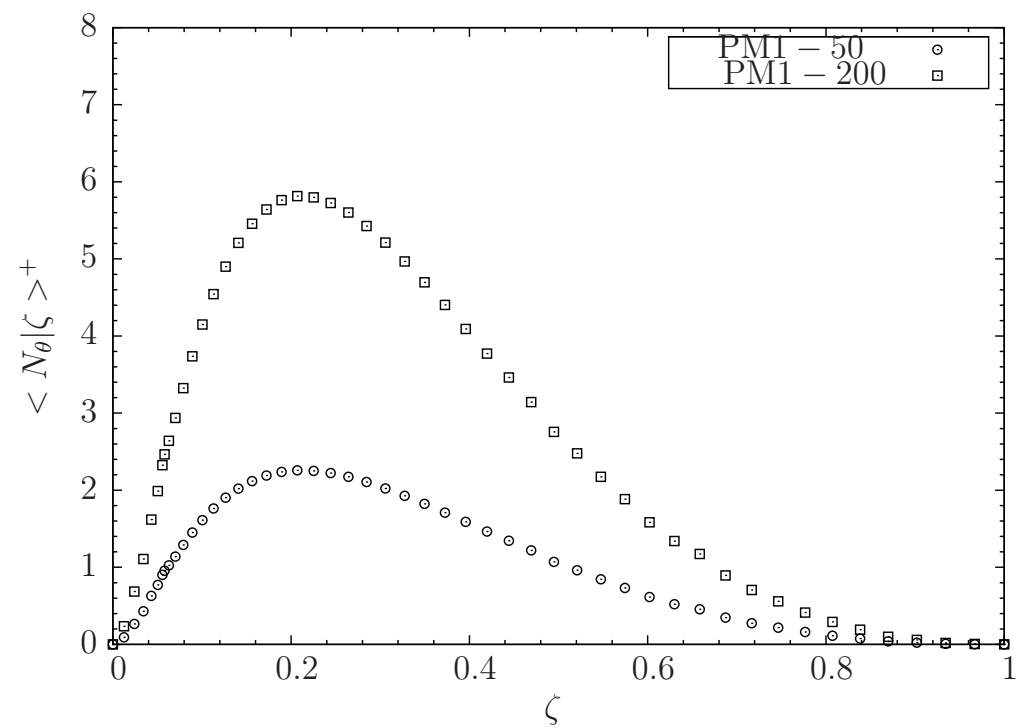

Figure 7.10: The typical variation of the conditional scalar dissipation rate, $\left\langle N_{\theta}^{+} \mid \zeta\right\rangle$, obtained in the CMC calculation for flames PM1-50 and PM1-200 at $\tilde{\theta}=0.55, x / D=8.5$. The values are non-dimensionalised using $S_{L}^{o}$ and $\delta_{L}^{o}$. 


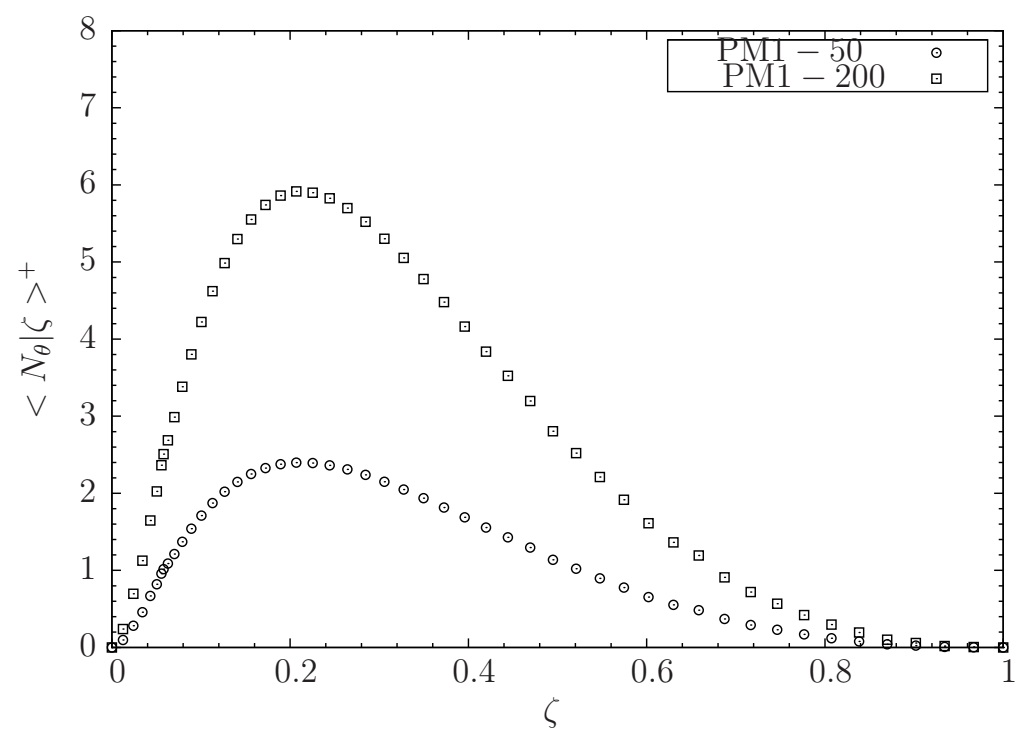

Figure 7.11: The typical variation of the conditional scalar dissipation rate, $\left\langle N_{\theta}^{+} \mid \zeta\right\rangle$, obtained in the CMC calculation for flames PM1-50 and PM1-200 at $\tilde{\theta}=0.55, x / D=10.5$. The values are non-dimensionalised using $S_{L}^{o}$ and $\delta_{L}^{o}$.

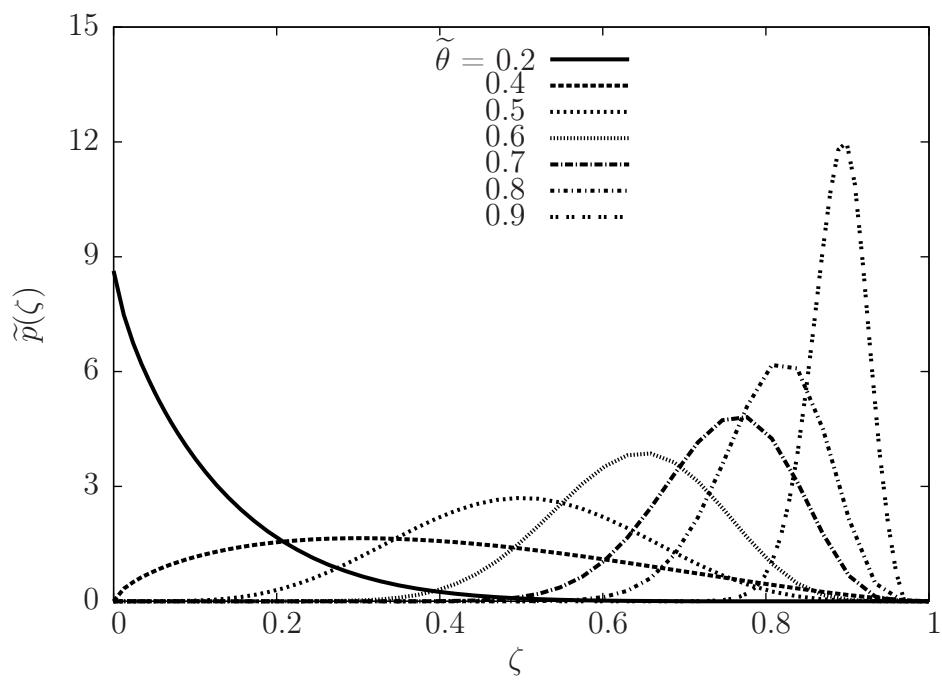

Figure 7.12: The variation of the Favre PDF of the progress variable, $\tilde{p}(\zeta)$, at selected locations of the flame brush of flame PM1-50. 


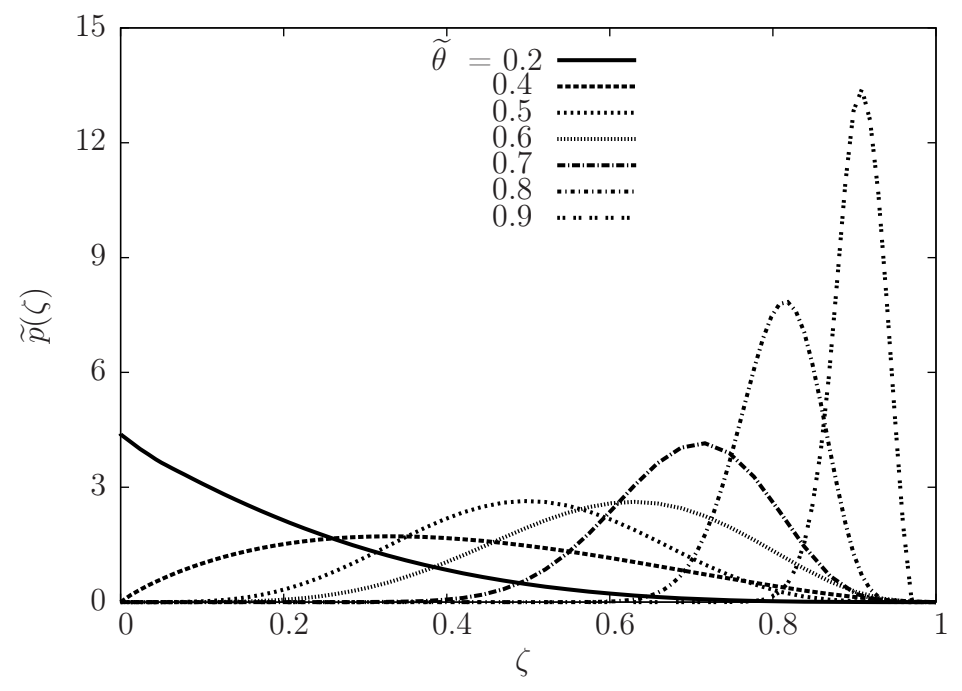

Figure 7.13: The variation of the Favre PDF of the progress variable, $\tilde{p}(\zeta)$, at selected locations of the flame brush of flame PM1-200.

\begin{tabular}{ccccc}
\hline \hline$\widetilde{\theta}$ & $\widetilde{\theta^{\prime \prime 2}}$ & $\widetilde{Z_{1}}$ & $\widetilde{Z_{2}}$ & $\widetilde{Z_{3}}$ \\
\hline 0.9 & 0.030 & 0.964 & 0.0181 & 0.017 \\
0.8 & 0.051 & 0.925 & 0.035 & 0.039 \\
0.7 & 0.094 & 0.883 & 0.054 & 0.062 \\
0.6 & 0.143 & 0.848 & 0.069 & 0.081 \\
0.5 & 0.141 & 0.809 & 0.087 & 0.103 \\
0.4 & 0.205 & 0.761 & 0.101 & 0.136 \\
0.2 & 0.155 & 0.620 & 0.126 & 0.252 \\
\hline
\end{tabular}

Table 7.3: Values of $\widetilde{\theta}, \widetilde{\theta^{\prime \prime 2}}, \widetilde{Z_{1}}, \widetilde{Z_{2}}$ and $\widetilde{Z_{3}}$ in flame PM1-200 for the PDFs shown in Fig. 7.13. 


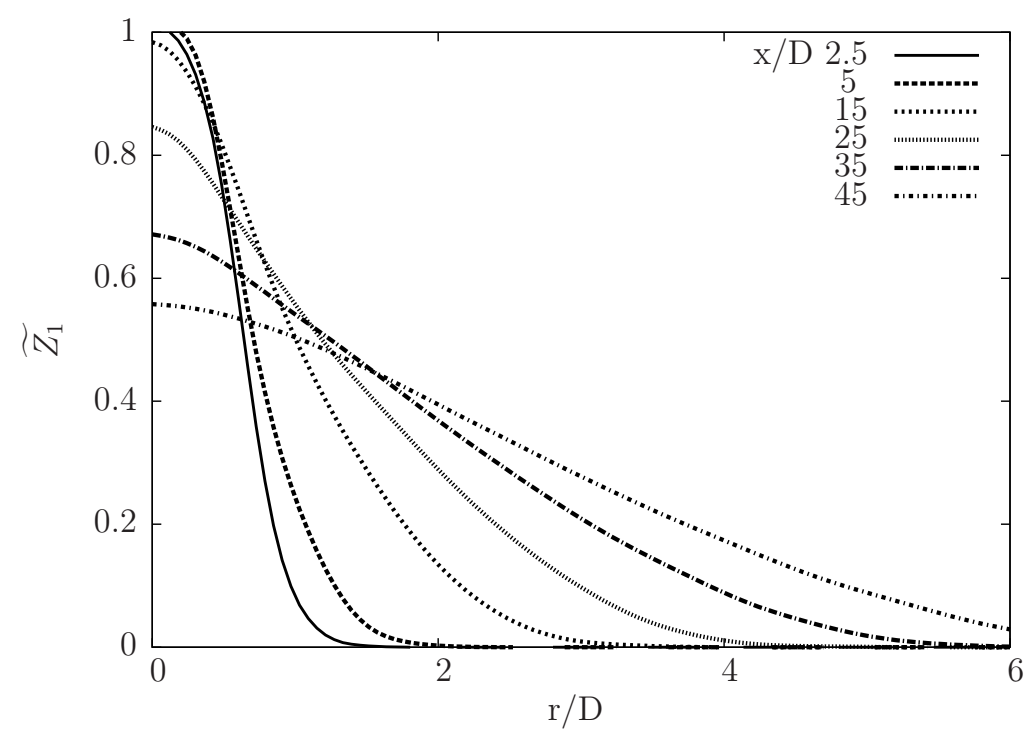

Figure 7.14: Computed radial variation of $\widetilde{Z 1}$ from the hot flow simulation at six axial locations for the conditions for flame PM1-200.

are shown in Figs. 7.14, 7.15, 7.16 and 7.17 respectively. Since these fields are nonreactive, convective and diffusive fields, they are expected to spread with down stream distance. The results in Figs. 7.14 to 7.17 clearly shown this behaviour. However, $\widetilde{Z_{3}}$ does not seem to decrease below one for $10 \leq r / D \leq 20$ for the axial locations shown in Fig. 7.17, this is because $x / d p 2=0.91$ for $x / D=45$. To see any significant drop from unity for $10 \leq r / D \leq 20$, one must go beyond $100 D$ axial distance. This point is clearly from Fig. 7.18 , showing a colour map of $\widetilde{Z_{3}}$. The spatial evolution of $\widetilde{Z_{1}}$ and $\widetilde{Z_{2}}$ is shown in Fig. 7.19. From these results, one observes that the mixing of these three streams is captured well. Figure 7.20 shows the spatial variation of $\widetilde{\theta}$ and $\widetilde{\theta^{\prime \prime 2}}$. Since the variance $\widetilde{\theta^{\prime \prime 2}}$ is produced by the chemical reaction and $\nabla \widetilde{\theta}$, one will observe a sharp increase in $\widetilde{\theta^{\prime \prime 2}}$ inside the flame brush. This behaviour is clearly in Fig.7.20

The variation of the center-line value of the mean and rms values of the axial velocity is shown in Figs. 7.4 and 7.5 respectively. These values are normalised 


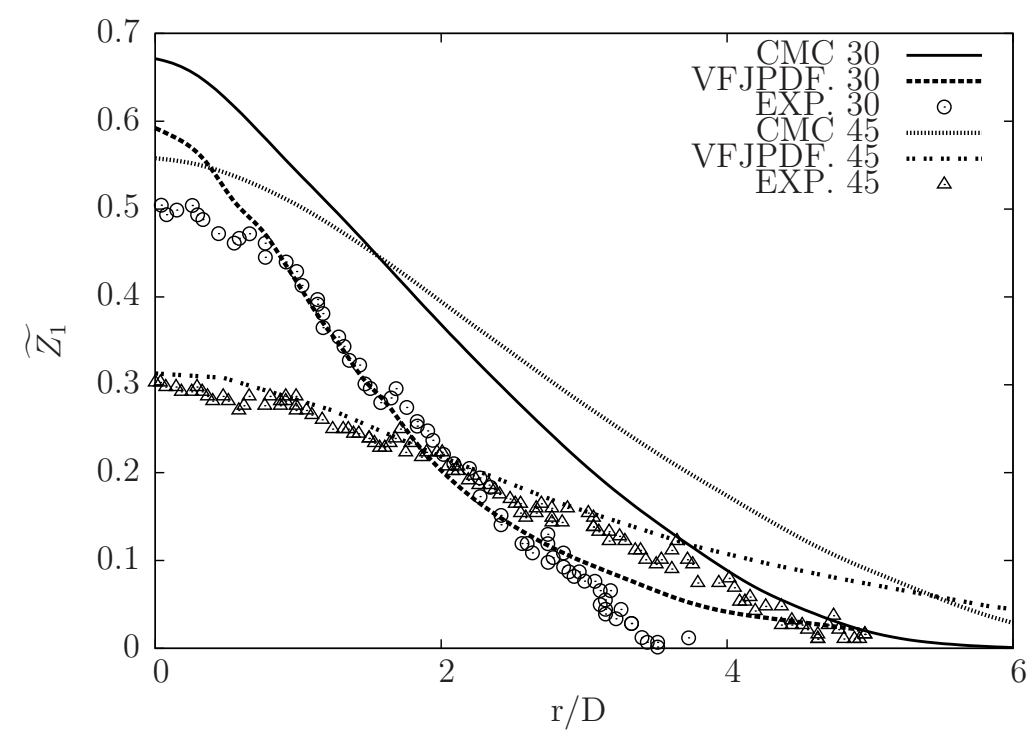

Figure 7.15: Computed radial variation of $\widetilde{Z 1}$ from the hot flow simulation are compared to PDF calculations [5] for flame PM1-200.

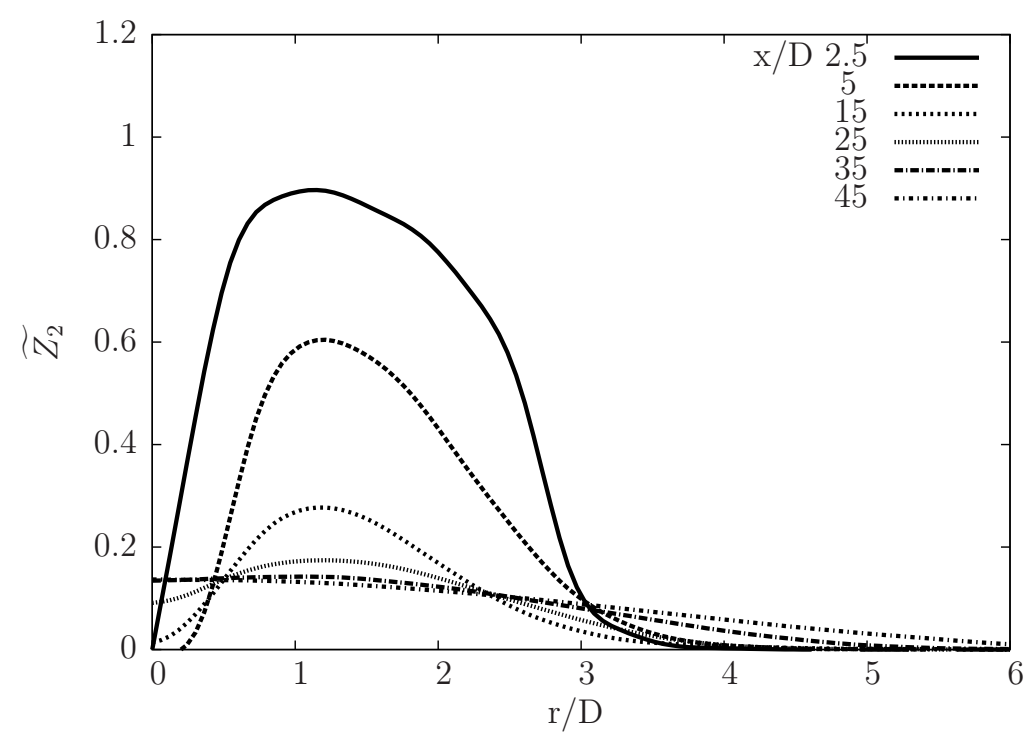

Figure 7.16: Computed radial variation of $\widetilde{Z 2}$ from the hot flow simulation at six axial locations for the conditions for flame PM1-200. 


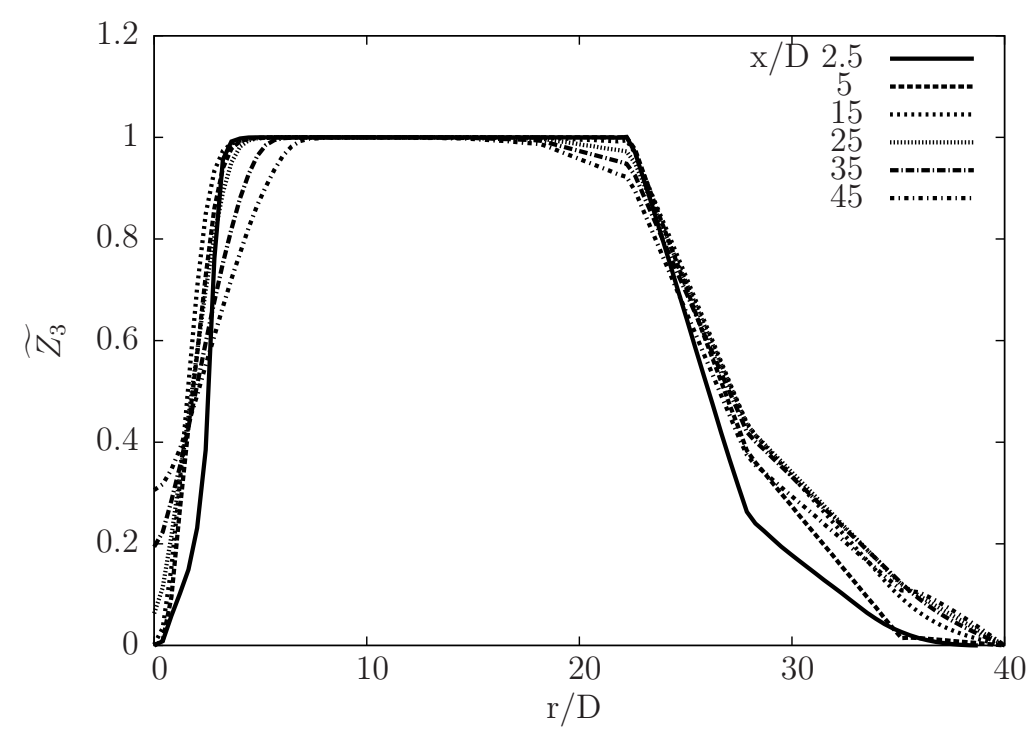

Figure 7.17: Computed radial variation of $\widetilde{Z 3}$ from the hot flow simulation at six axial locations for the conditions for flame PM1-200.

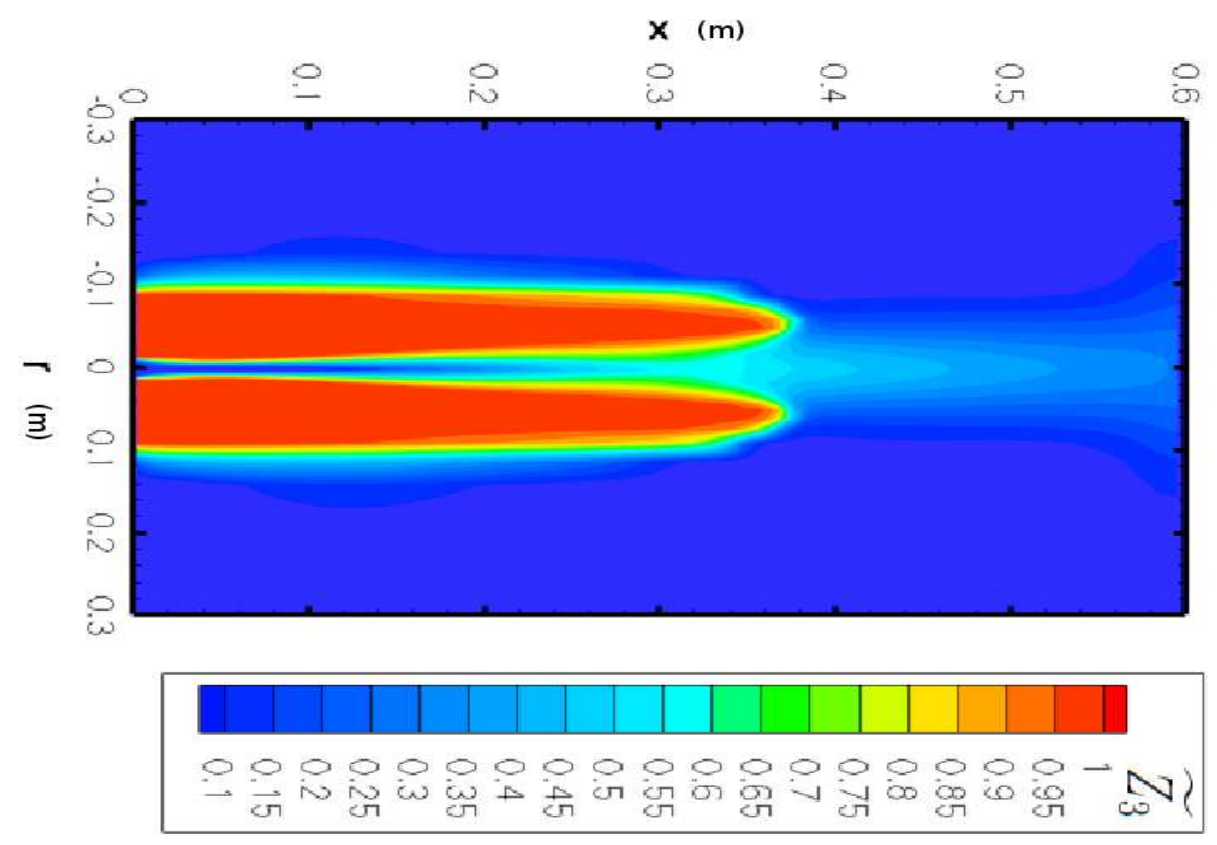

Figure 7.18: Contours of $\widetilde{Z_{3}}$ for flame PM1-200 

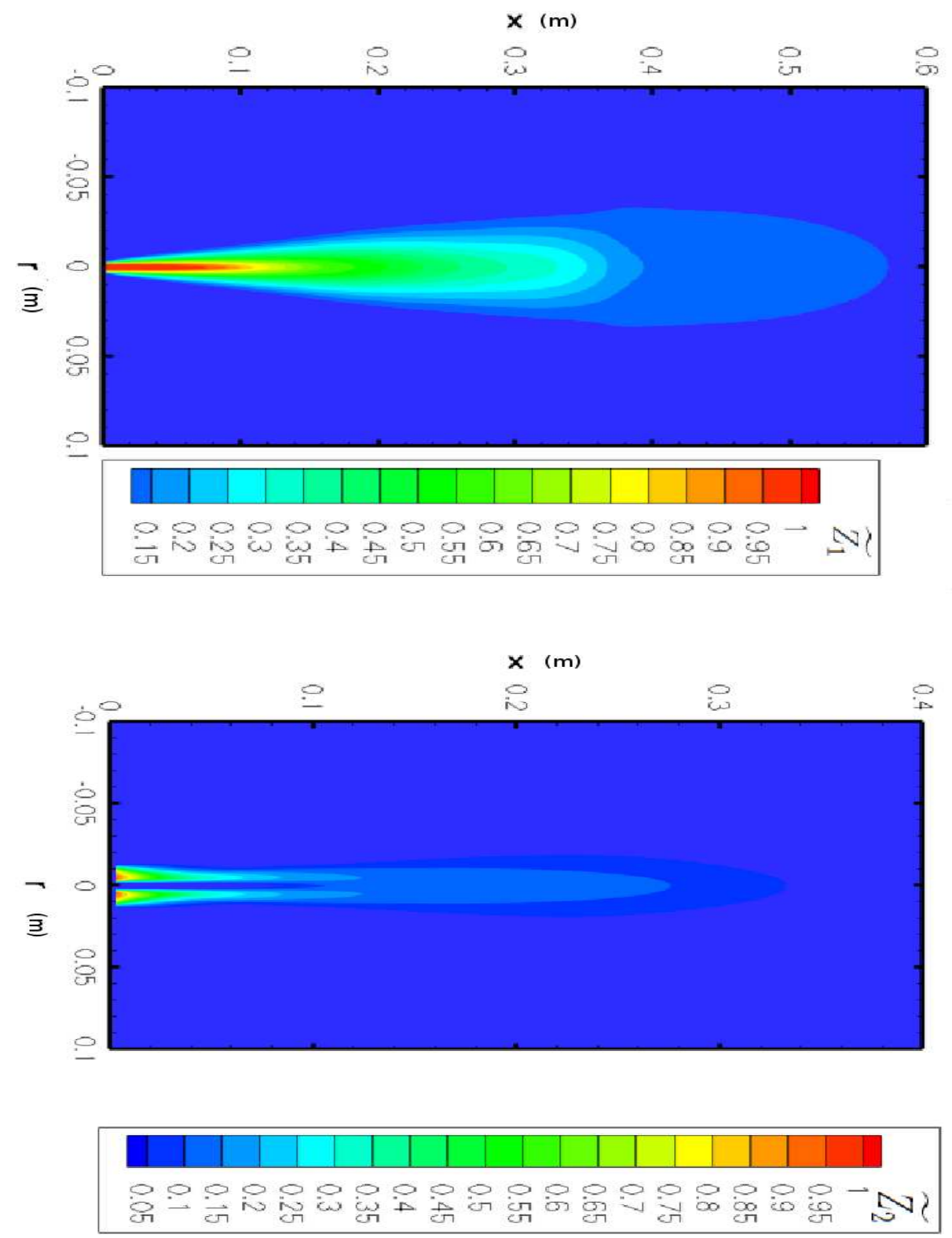

Figure 7.19: Contours of $\widetilde{Z_{1}}$ and $\widetilde{Z_{2}}$ for flame PM1-200 

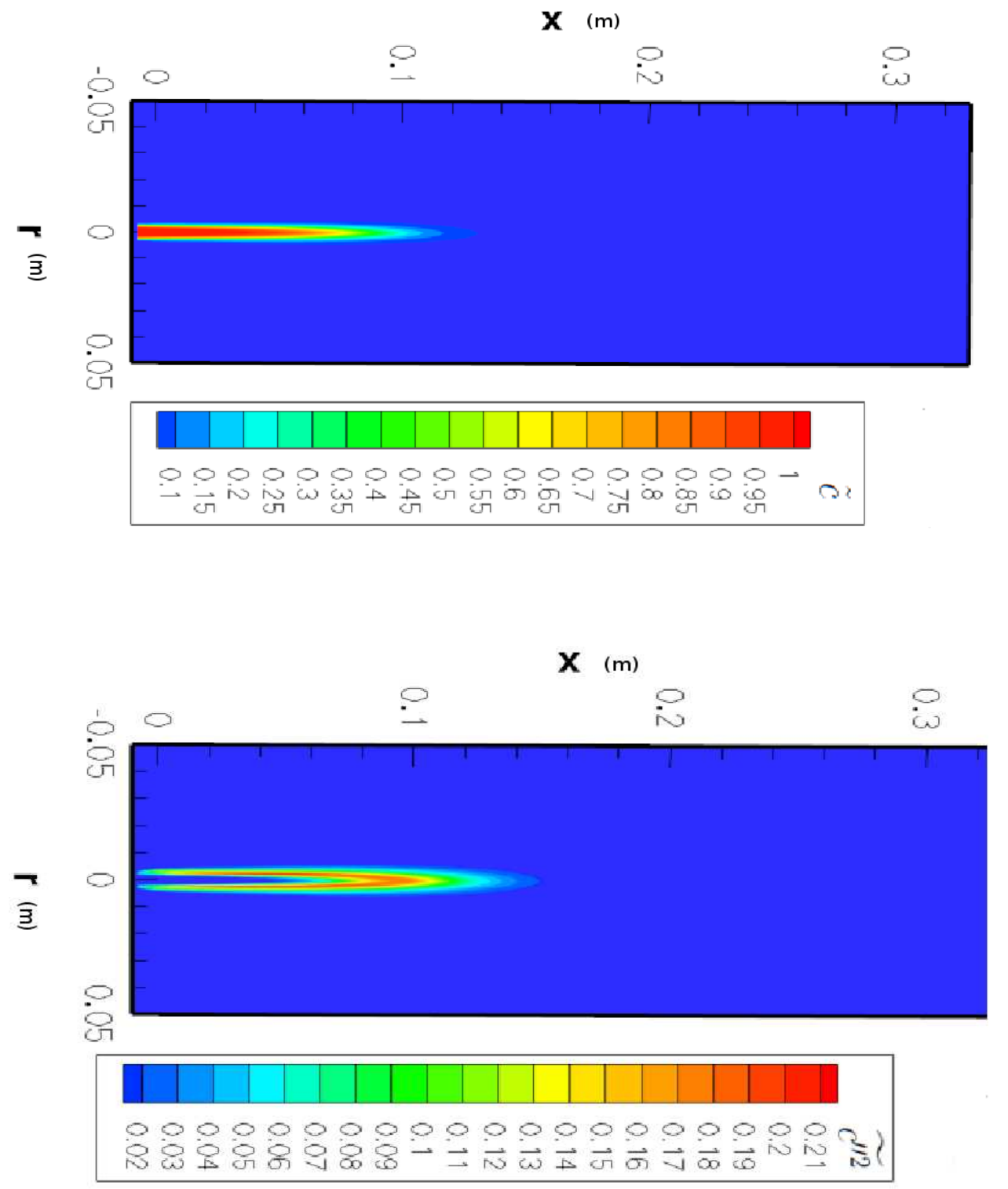

Figure 7.20: Contours of the mean progress variable and its variance for flame PM1-200 
using the bulk mean value at the main jet inlet, $U_{0}$. The computed values are compared to the measurements in these figures. The agreement in the mean values at locations close to the fuel nozzle is excellent. Further down stream, particularly for $x / D>20$, the computed values are over-predicted for flame PM1-200, while the agreement is improved for flame PM1-50 which has a lower Re number. The rms values for both flames show some discrepancies at all axial locations.

In the radial direction, the computed mean velocity using the CMC for flame PM1-200 is over predicted for all axial locations as shown in Fig. 7.21. A good agreement is observed for $x / D=5$ and 55. However, for flame PM1-50, a good agreement with the experimental measurements is observed for all axial locations as shown in Fig. 7.22. The rms velocity for flame PM1-200 is shown in Fig. 7.23, and the CMC values are in reasonable agreement with the experimental measurements. While, for flame PM1-50 there are some discrepancies with the experimental measurements as in Fig. 7.24. These are typical for $k$ - $\varepsilon$ turbulence model used in the calculations. These behaviours for the computed velocities are very similar to those reported by Dunn et al. [6] and Dunn [4] using transported PDF modelling. This comparison is also shown in Figs 7.21 to 7.24.

Figures 7.25 and 7.26 show the radial variation of mean temperature from the CMC calculations of flames PM1-200 and PM1-50, respectively. The influence of the pilot gases on the main jet mixture is well captured. The adiabatic flame temperature $\approx 1480 \mathrm{~K}$, is predicted well inside the flame brush at $r / D=0.7$ at $x / D=2.5$, and it is consistent with these reported in the experiment [6]. The high temperature between $r / D=1$ to 2.5 , indicate that the reaction of the main jet is influenced mainly by the hot pilot temperature. Also, the effect from the 
lean hot co-flow stream is well captured at $r / D=2.8$ and beyond. This effect is captured as a drop in the temperature between the stoichiometric pilot and the lean co-flow streams. The drop in the mean temperature to a value of about $1300 \mathrm{~K}$ near $r / D=3$ for $x / D=2.5$ could be due heat loss to the burner, which is not included in the CFD model. This heat loss effect is shown clearly by Rowinski and Pope [5], however, it is also noted in that study that the sensitivity of the computed results at locations beyond $x / D=25$ is small.

The radial variation of the mean temperature shown here for PM1-200 is very similar to that shown by Rowinksi and Pope [5], Dunn et al. [6] and Dunn [4] computed using different approaches and modellings for transported PDF methodology. The radial variation of the calculated mean temperature is shown in Fig 7.26 for flame PM1-50 and the agreement with the measured values is observed to be very good, when compared to the PM1-200 flame. This is not surprising because the turbulence model used in this study seems to predict the turbulence quantities better for PM1-50 flame as noted earlier. Since the CMC sub-models feed on the predicted turbulence quantities, one observes a good prediction for flame PM1-50. The relative behaviour of the computed and measured mean temperature for flame PM1-50 is very similar to that reported by Rowinksi and Pope [5], Dunn et al. [6] and Dunn [4]. This comparison is also shown in Figs 7.25 to 7.26

The radial variation of the mean mass fractions of $\mathrm{CO}$ and $\mathrm{OH}$ computed using the CMC are compared to the experimental measurements and PDF computations $[4 ; 6]$ in Figs. 7.27, 7.28, 7.29 and 7.30. Note that the values of CO and $\mathrm{OH}$ are multiplied by $10^{3}$ and $10^{6}$, respectively. Except for the near field location, $x / D=2.5$, the mean $\mathrm{CO}$ and $\mathrm{OH}$ mass fractions are over predicted for 
flame PM1-200 at all axial location as in Fig 7.27 and 7.29. The radial variation of the mean $\mathrm{CO}$ and $\mathrm{OH}$ mass fractions are captured well for flame PM1-50. The mean CO mass fraction for PM1-50 is in very good agreement with the experimental measurements except for location $x / D=25$, while the mean $\mathrm{OH}$ mass fraction is in good agreement only in the near field location, $x / D=2.5$. The comparisons between the CMC and measured values are similar to those observed for transported PDF calculations, as noted in Figs 7.27 to 7.30

The comparison of computed and measured CO shown in Fig 7.28 for flame PM1-50 is slightly better than those shown by Rowinksi and Pope [5], Dunn et al. [6] and Dunn [4] from calculations using the PDF methodologies. However, the comparison shown here for flame PM1-200 is somewhat similar to that reported in $[5]$ and $[4 ; 6]$. It is interesting to note a similar behaviour for the reactive scalar mean mass fractions and temperatures, despite a vast difference in the modelling methods used. There is gross over prediction of mean $\mathrm{CO}$ and $\mathrm{OH}$ mass fractions and temperature for flame PM1-200. Rowinksi and Pope [5] showed that this is because the reaction rates in flame PM1-200 are reduced and the reaction rates have to be slowed down by a factor of 5 in their PDF calculation to obtain a good match with measured mean mass fraction values and temperature. This observation seem to apply for the CMC calculations reported here, but further calculations are required to verify this. 

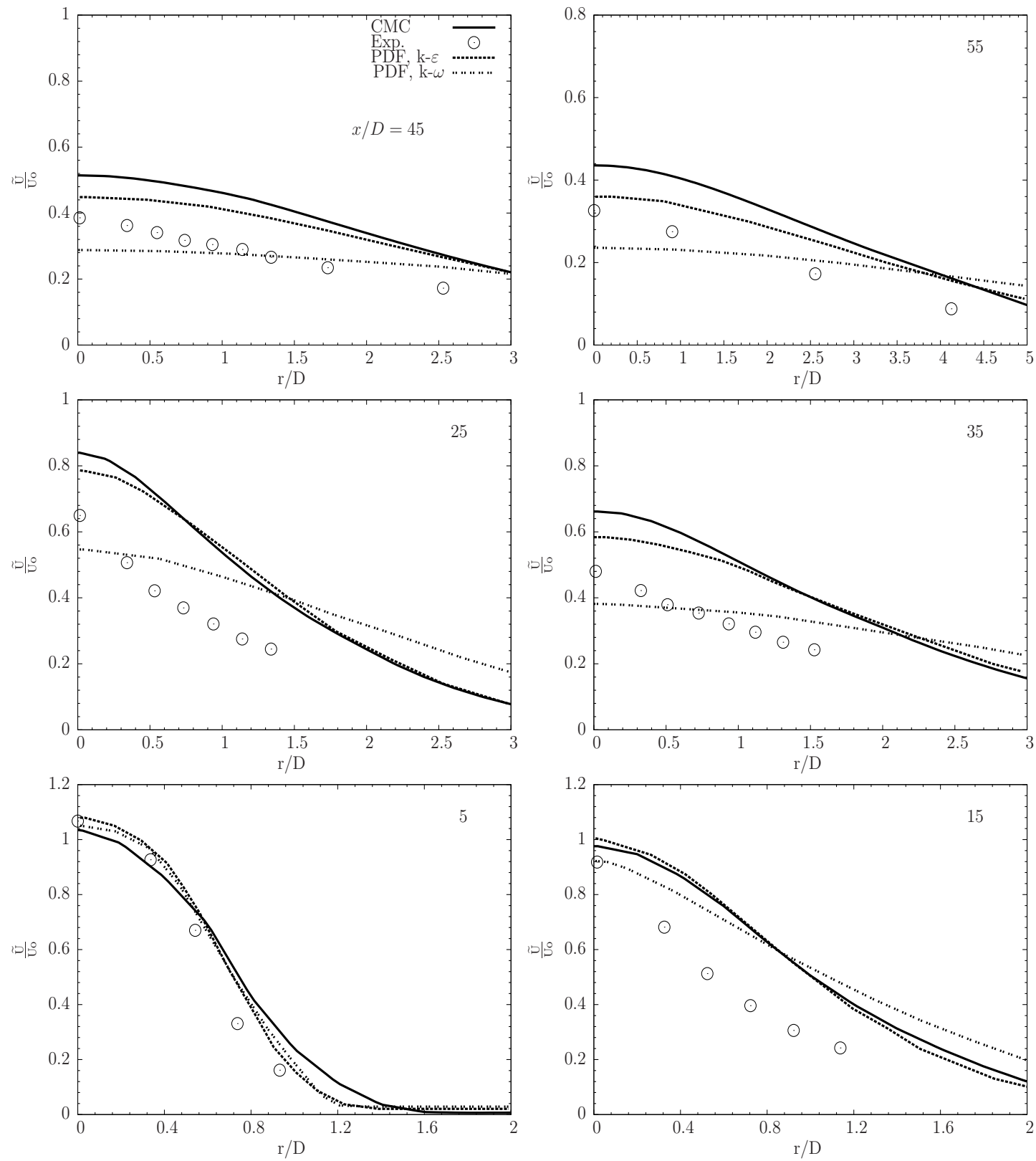

Figure 7.21: The mean velocity from the CMC calculations at six axial locations are compared to the experimental data [4] and PDF calculations, $k-\varepsilon$ and $k-\omega[6]$ for flame PM1-200. 

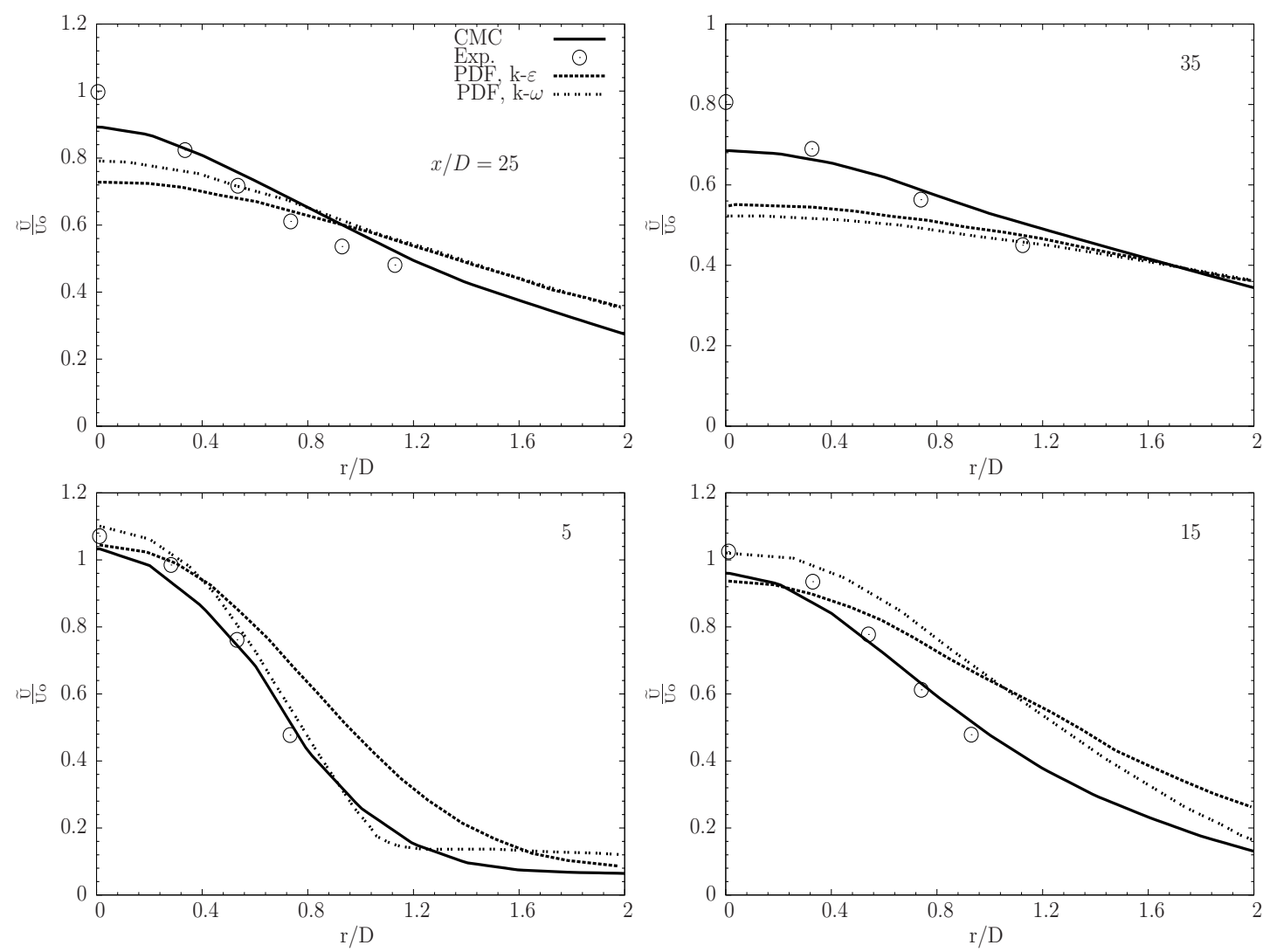

Figure 7.22: The mean velocity from the CMC calculations at four axial locations are compared to the experimental data [4] and PDF calculations, $k-\varepsilon$ and $k-\omega[6]$ for flame PM1-50. 

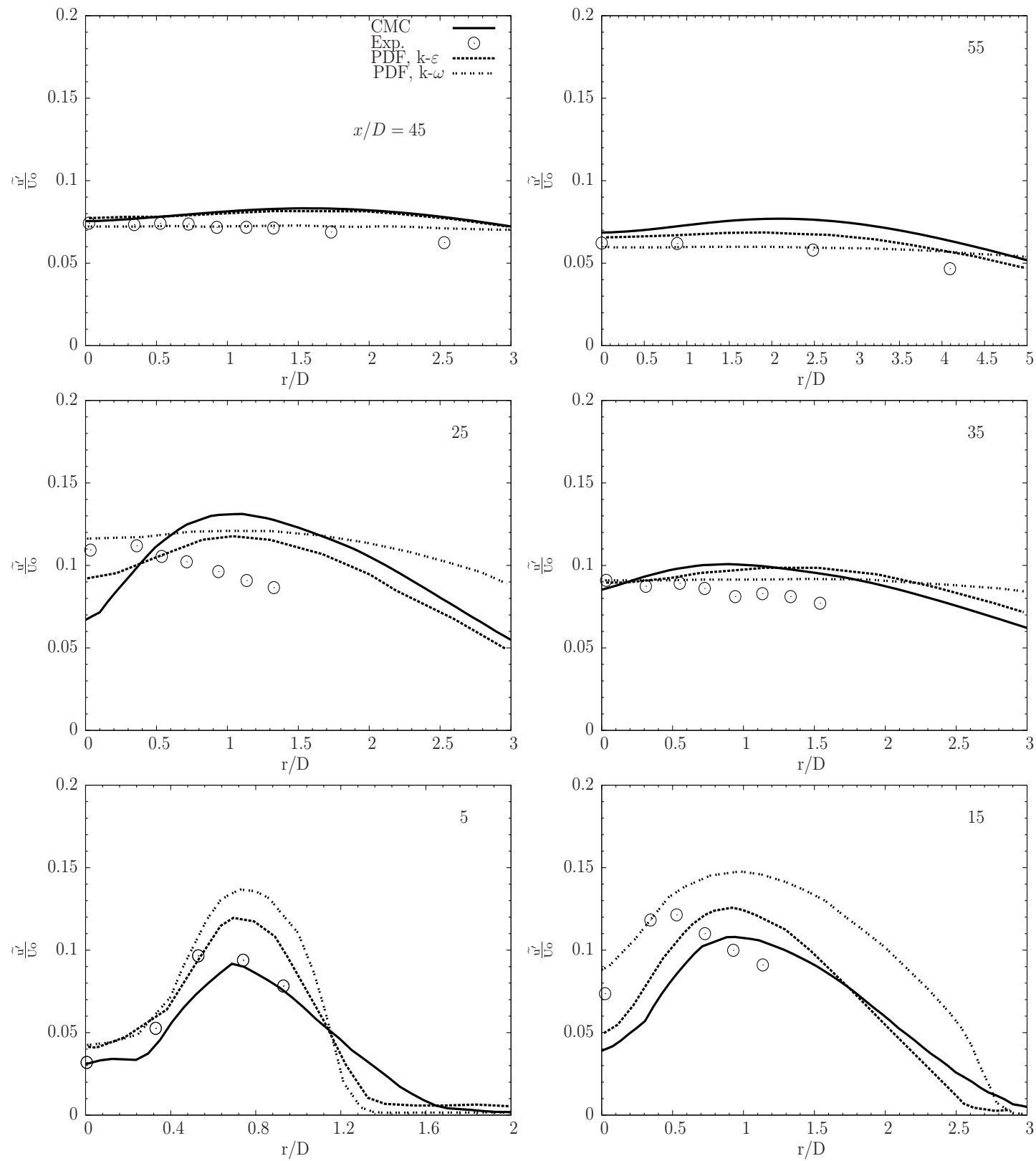

Figure 7.23: The mean rms velocity from the CMC calculations at six axial locations are compared to the experimental data [4] and PDF calculations, $k-\varepsilon$ and $k-\omega[6]$ for flame PM1-200. 

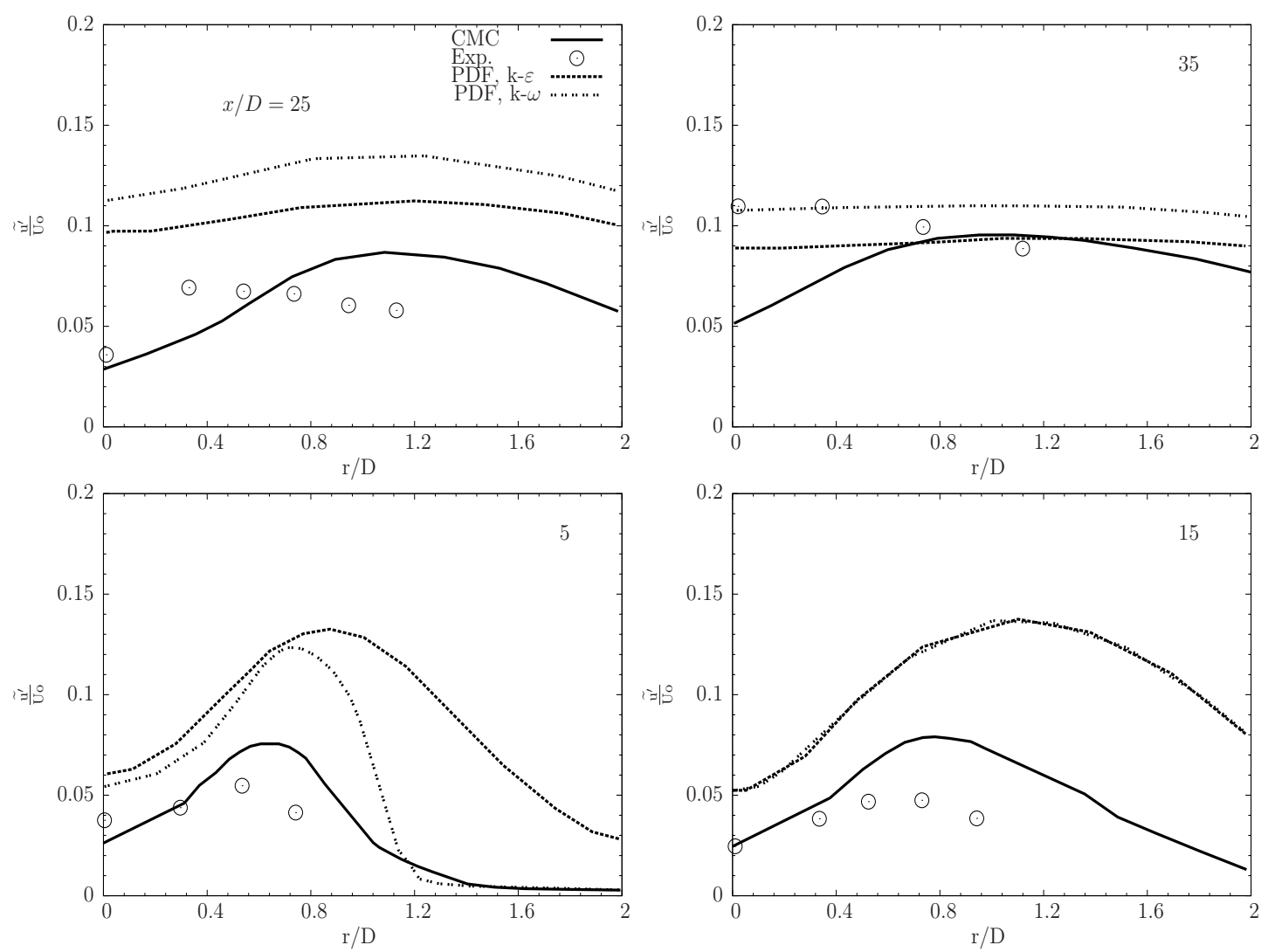

Figure 7.24: The mean rms velocity from the CMC calculations at four axial locations are compared to the experimental data [4] and PDF calculations, $k-\varepsilon$ and $k-\omega[6]$ for flame PM1-50. 

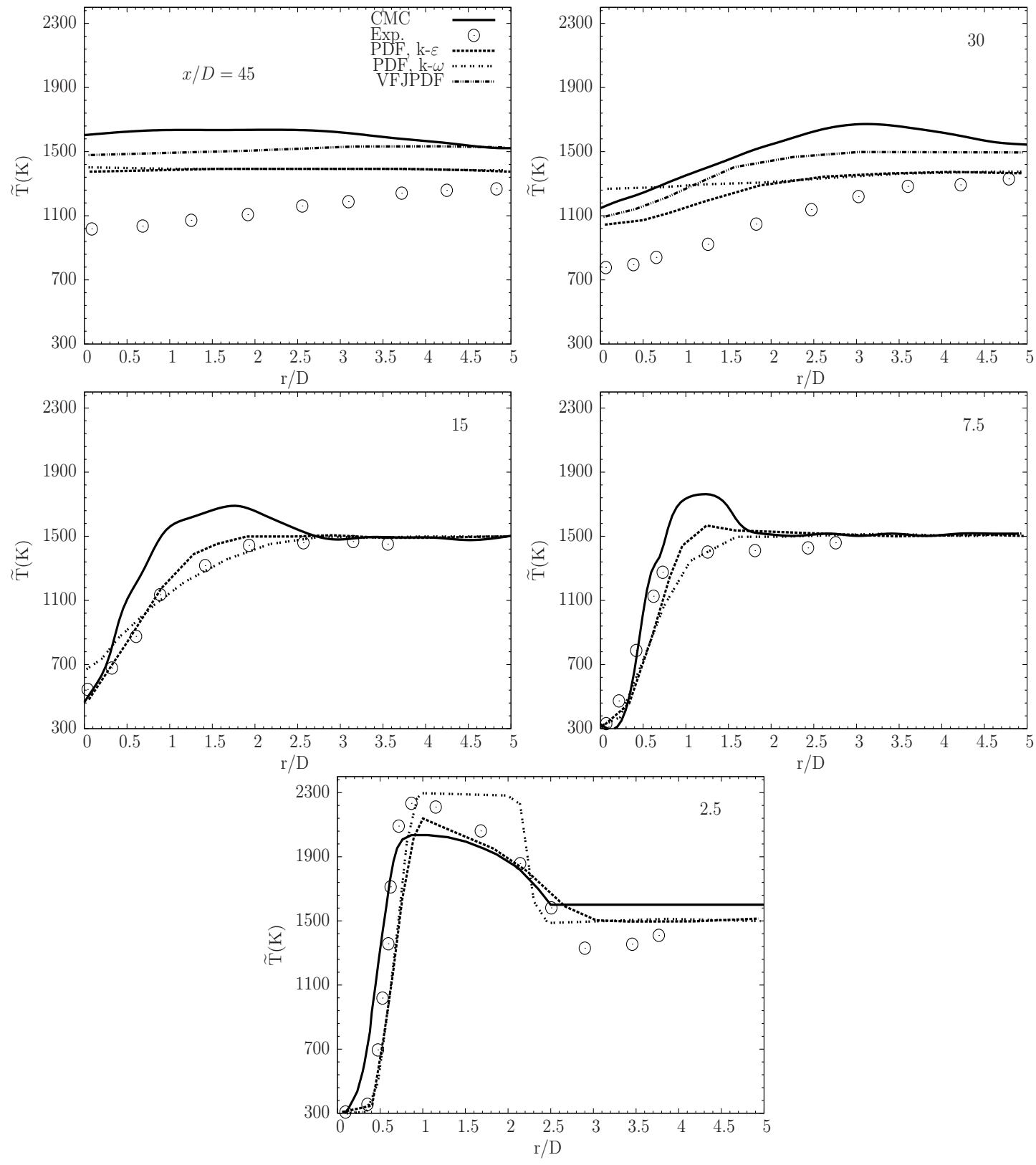

Figure 7.25: The mean temperature from the CMC calculations at four axial locations are compared to the experimental data [4] and PDF calculations, $k-\varepsilon$ and $k-\omega[6]$ and VFJPDF [5] for flame PM1-200. 

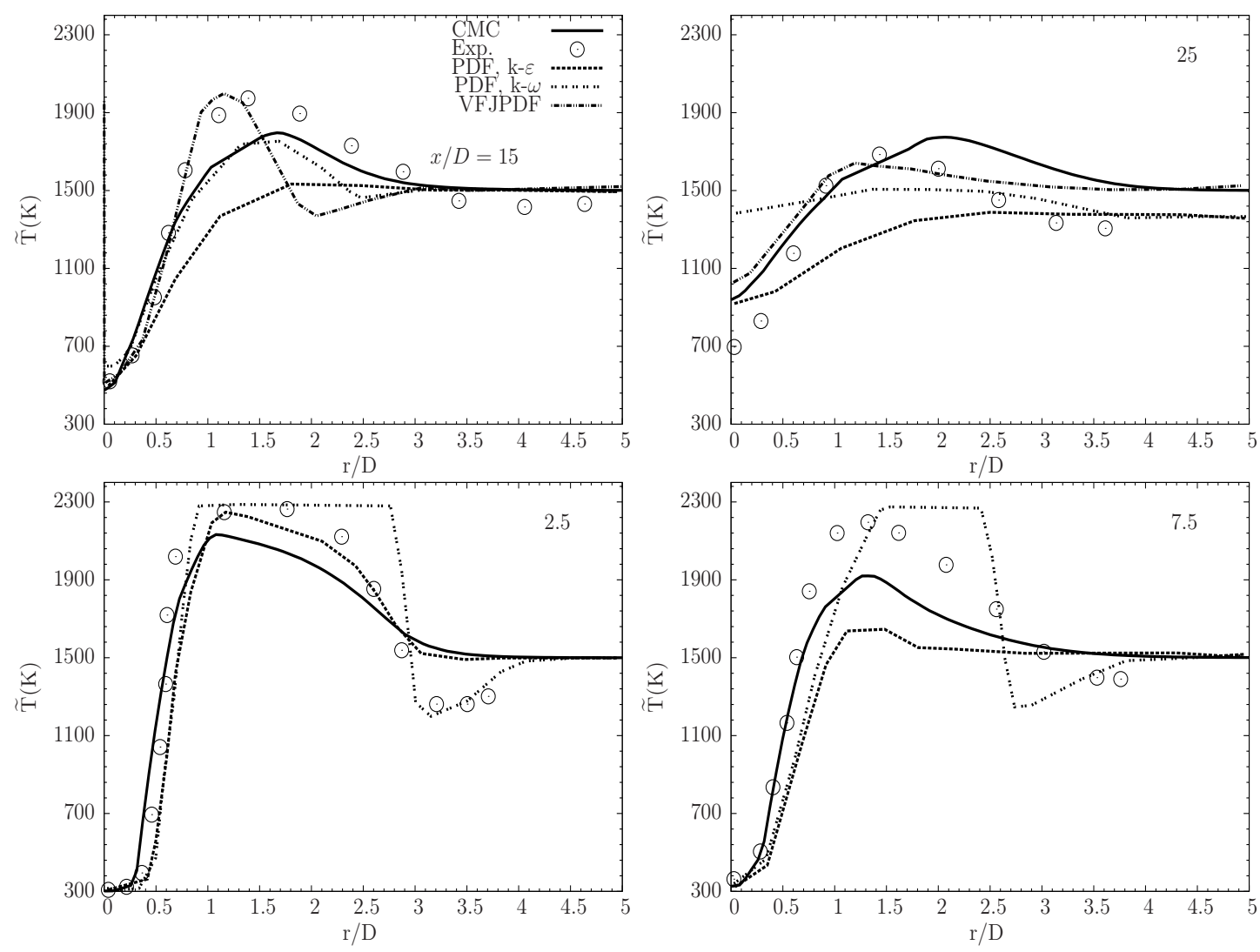

Figure 7.26: The mean temperature from the CMC calculations at four axial locations are compared to the experimental data [4] and PDF calculations, $k-\varepsilon$ and $k-\omega[6]$ and VFJPDF [5] for flame PM1-50. 

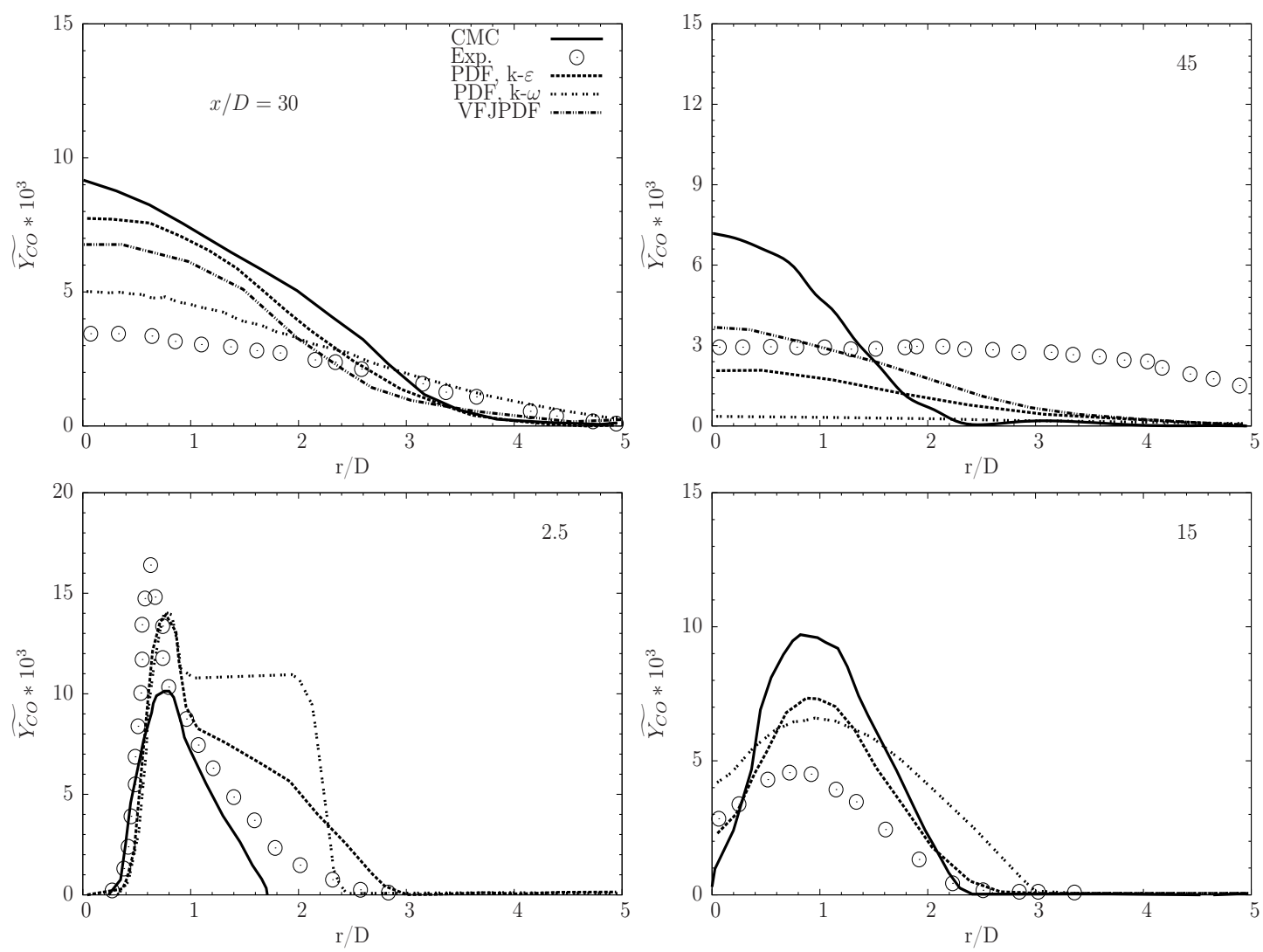

Figure 7.27: The mean $\widetilde{\mathrm{CO}} \times 10^{3}$ from the CMC calculations at four axial locations are compared to the experimental data [4] and PDF calculations, $k-\varepsilon$ and $k-\omega[6]$ and VFJPDF [5] for flame PM1-200. 

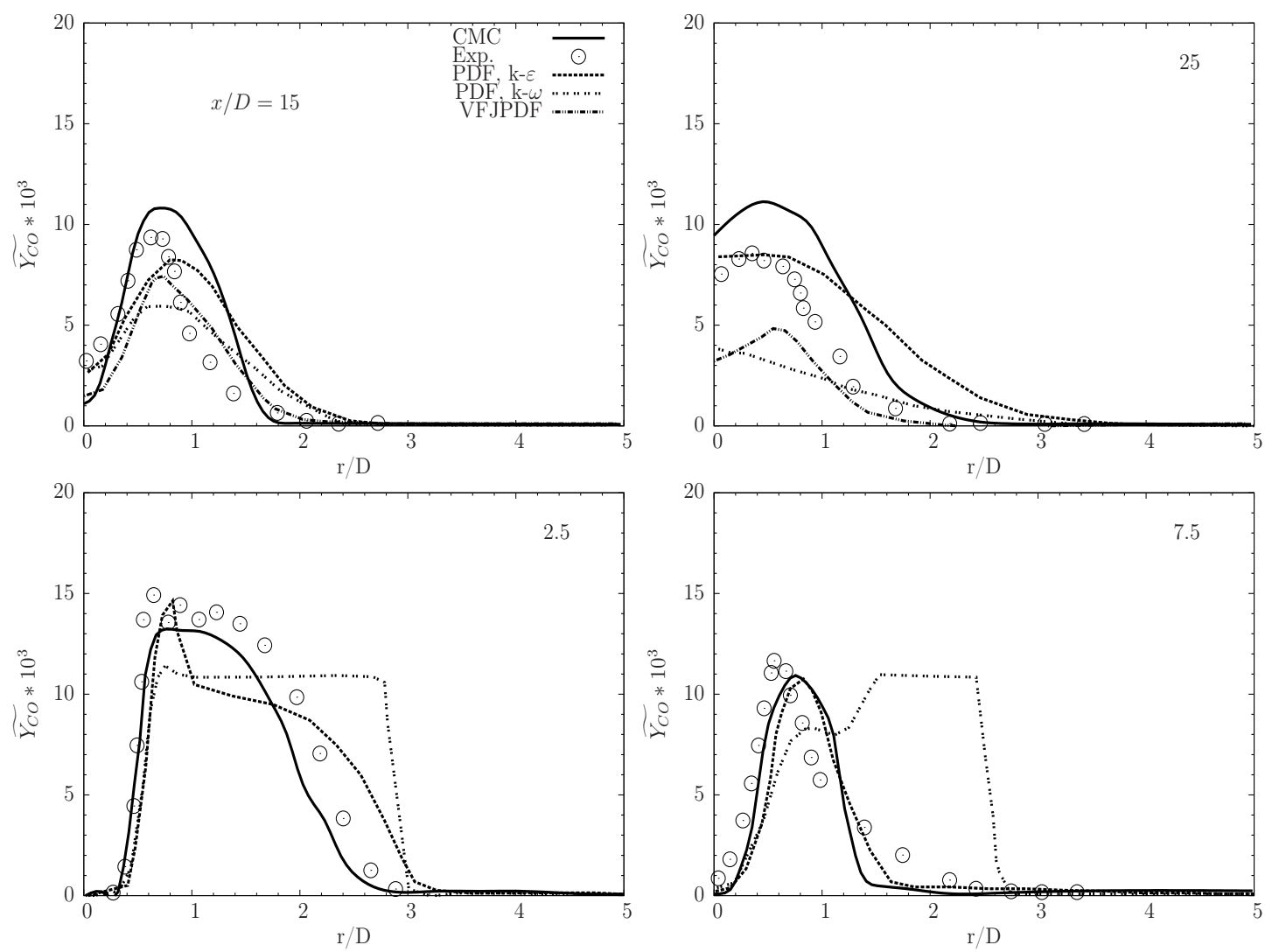

Figure 7.28: The calculated mean $\widetilde{\mathrm{CO}} \times 10^{3}$ mass fraction from CMC calculations at four axial locations are compared to the experimental data [4] and PDF calculations, $k-\varepsilon$ and $k-\omega[6]$ and VFJPDF [5] for flame PM1-50. 

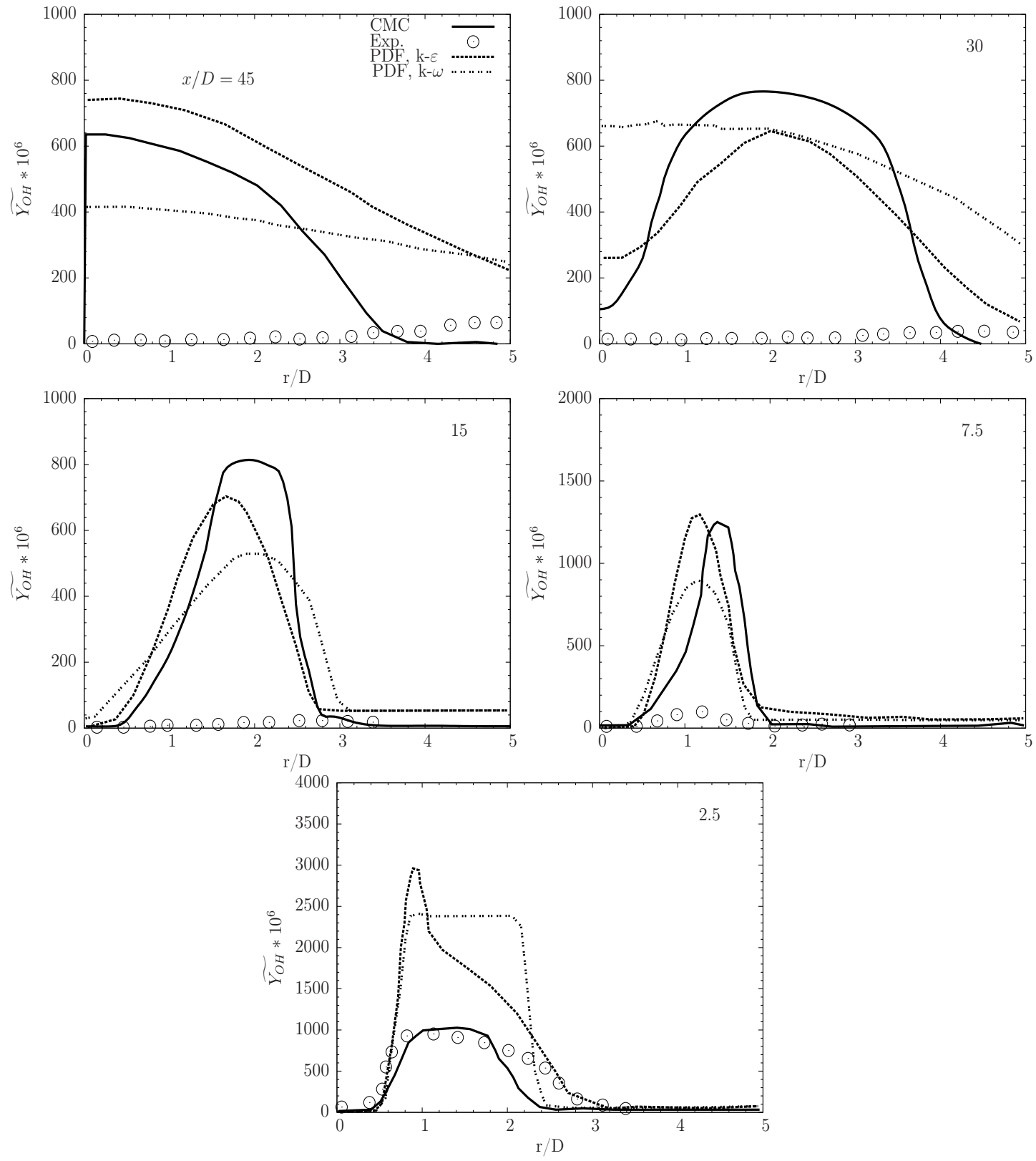

Figure 7.29: The calculated mean $\overline{\mathrm{OH}} \times 10^{6}$ from CMC calculations at five axial locations are compared to the experimental data [4] and PDF calculations, $k-\varepsilon$ and $k-\omega[6]$ for flame PM1-200. 

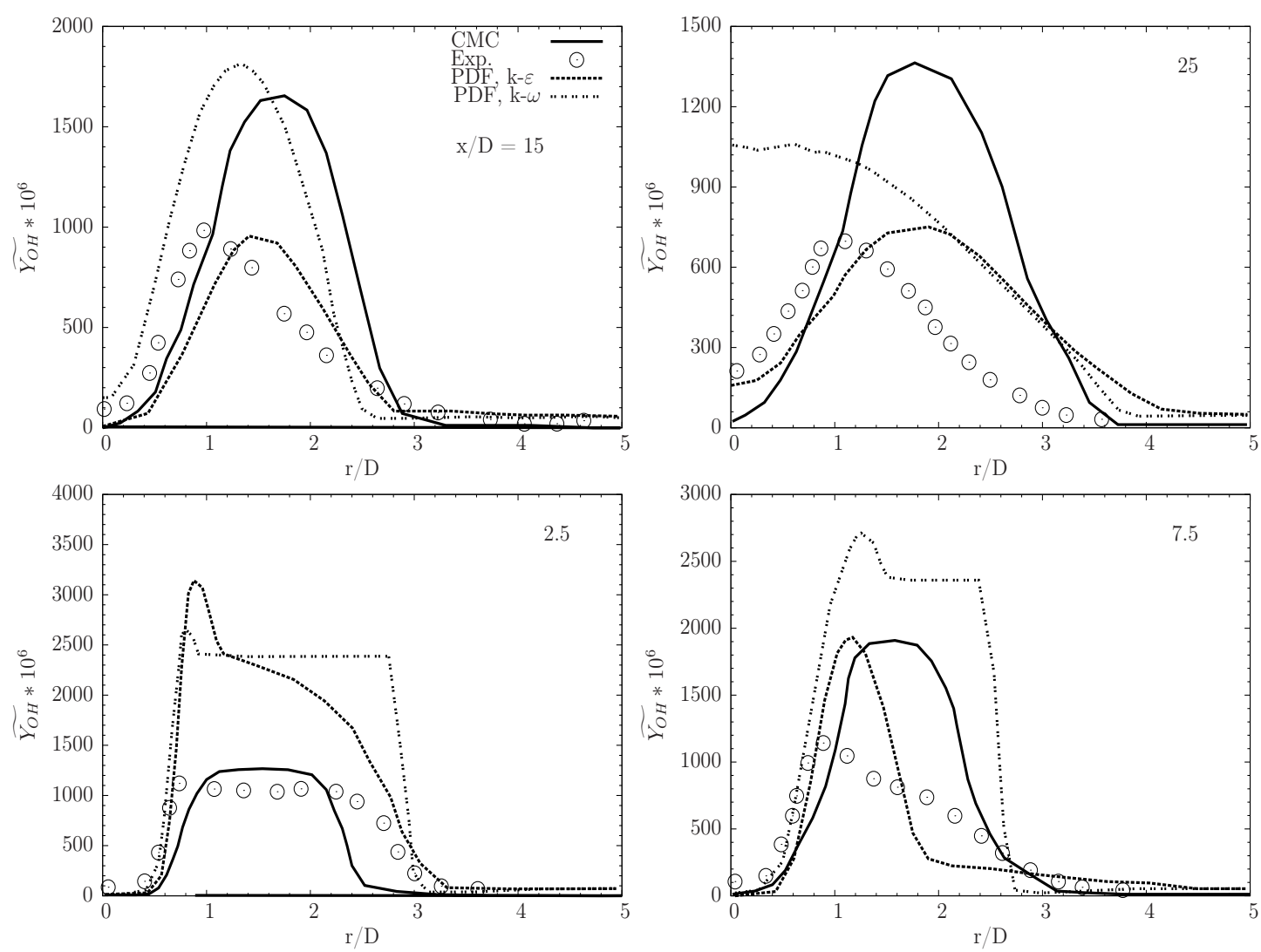

Figure 7.30: The calculated mean $\overline{\mathrm{OH}} \times 10^{6}$ from CMC calculations at four axial locations are compared to the experimental data [4] and PDF calculations, $k-\varepsilon$ and $k-\omega[6]$ for flame PM1-50. 


\section{Chapter 8}

\section{Summary and Conclusions}

\subsection{Conclusion From This Work}

The over all aim of this study was to explore the conditional moment closure method for turbulent lean premixed flames. Specifically, to predict pollutants such as $\mathrm{NO}_{\mathrm{x}}, \mathrm{CO}$ in turbulent lean premixed flames. These species have slow time scales and thus flamelet type approach may be insufficient. The CMC and the transported JPDF are alternative approaches. In this study, the CMC is considered, which was used and validated mainly for non-premixed flames in the past studies $[30 ; 31 ; 32 ; 33 ; 34 ; 35 ; 36]$. The main difficulties in applying CMC to premixed flames is due to the conditional scalar dissipation rate in the CMC transport equations, see Eq. 3.3. This term is crucial in the CMC method and the accuracy of the method depends on accurate modelling for this term. The scalar dissipation rate signifies the local mixing rate of reacted and unreacted mixtures inside the turbulent flame brush and this mixing rate will dictate the chemical reaction rate.

In this study, two methods to find a closure for the conditional scalar dissipation rate was tested in chapter 4. One method, see Eq. 4.11, is based on the 
mathematical techniques for finding a solution for ill-posed problem and involves an integral equation relating the mean dissipation rate to the conditional dissipation rate. The second method uses a simple algebraic model, proposed in an earlier study [79]. These two models were validated using two different DNS data sets; one for stoichiometric and another one for lean turbulent premixed flames. The results showed that the simple algebraic model was in better agreement with the DNS data and the results obtained using the first method showed a high sensitivity to the initial values for the conditional dissipation rate. Thus, the algebraic model is chosen as a closure for the conditional scalar dissipation rate in this study.

The CMC sub-models for the premixed flames, discussed in chapter 3, were implemented along with the conditional scalar dissipation rate model in a threedimensional CFD code. The CFD code is based on the RANS approach using the standard $k-\varepsilon$ turbulence modelling. This code, originally developed for nonpremixed combustion [127], was modified for the premixed flames by including transport equations for the progress variable, $\tilde{c}$, and its variance $\widetilde{c^{\prime \prime 2}}$ along with suitable closures for the source/sink terms, $\overline{\dot{\omega}_{c}}, \overline{c^{\prime \prime} \dot{\omega}_{c}}$ and the mean scalar dissipation rate $\widetilde{\epsilon_{c}}$. The closures used for the terms related to the chemical reactions are consistent with the CMC methodology as described in section 5.2. The RANSCMC equations are solved using finite volume methodology. The details are presented in chapter 5 .

The RANS-CMC method is used to compute stoichiometric [2] and lean pilot [3] stabilized Bunsen flames, five flames in total. A first order closure is used for the conditional mean reaction rate. The PDF of the progress variable is obtained using a presumed shape with a Beta function. The GRI-mech. 3.0 and 
the skeletal mechanism of Smooke are used for modelling the chemical kinetics for the stoichiometric flames to study the effects of chemical mechanisms on the computed results. The results are presented in chapter 6. The GRI-mech 3.0 is used for the lean flames and the results are discussed in chapter 7. The turbulence models and the boundary conditions used are assessed first by simulating the nonreacting flow where the computed mean velocity and turbulent kinetic energy agree reasonably well with the measurements for all the flames studied. As for the reacting flow, the mean velocity and mean turbulent kinetic energy were satisfactory and acceptable for both stoichiometric and lean flames. The influence of chemical kinetic mechanism and $e_{Q_{\alpha}}$ term on the reacting flow were assessed using three simulations for the flame F1 of Chen et al.[2]; one using the skeletal mechanism, the second simulation using the GRI-mech and, the third simulation using GRI-mech and including $e_{Q_{\alpha}}$ term. The results showed that the effect of $e_{Q_{\alpha}}$ was in general negligible and the effect of chemical mechanism was significant only for minor species.

For the stoichiometric flames reported in [2], the computed mean mass fraction of methane and normalised temperature are in reasonable agreement with measurements for all the three flames. The predicted major species mass fractions are also in reasonable agreement with the experimental data. However, the level of agreement improves as the overall Damköhler number increases. As for the minor species reported in the experiments are concerned, $\mathrm{CO}$ is over predicted, $\mathrm{H}_{2}$ is under predicted and $\mathrm{OH}$ is observed to agree quite well with the measurements at all axial locations for all the three flames in [2]. The reason for the discrepancies observed for CO mass fraction is unclear, although the level of disagreement is similar to that reported in earlier studies $[68 ; 79 ; 118 ; 119 ; 120 ; 121]$ using 
other combustion modelling approaches for these experimental flames. This gives confidence on the models and methodology used in this study.

A comparison of peak $\mathrm{CO}$ values at a given axial location show a weak sensitivity to the turbulence Reynolds and Damköhler numbers, at least for the range considered in the experimental studies, but the CMC results suggest a drop in this value as the Damköhler number increases, which is in agreement with our expectation.

Although the sensitivity of $\mathrm{CO}$ to the choice of the progress variable was shown to be small in an earlier study using flamelets based modelling [79], the effects of changing the conditioning variable definition on the CMC results are yet to be explored. The effects of other modelling, for example the counter-gradient scalar flux, on the CMC prediction need to be studied.

For the lean flames, the computed mean temperature is in good agreement for the flames PM1-50 for all axial locations. As for the flame PM1-200, the agreement was better near the fuel exit, while in the down stream distance, the agreement was less. However, the influence of the pilots gases and the adiabatic temperature were well captured. The predicted minor species $\mathrm{CO}$ and $\mathrm{OH}$ mass fractions were in reasonable agreement in the flame PM1-50, and the agreement was less for the flame PM1-200. The observed agreement for the PM1-200 flame was similar to those observed in earlier studies using transported joint PDF approach $[3 ; 4]$ and $[5]$. 


\subsection{Future Work}

There are several points, which can be explored in future studies, some of these points are:

1. The unconditional and conditional turbulent scalar flux is modeled using the classical gradient transport hypothesis in this study. As theoretical analysis and experimental studies pointed out the existence of both gradient and counter gradient fluxes and their co-existence in turbulent premixed flames. It is worthwhile to explore CMC with scalar flux models which can include, both gradient and counter gradient fluxes.

2. The progress variable based on fuel mass fraction is used as the conditioning variable in this study. Thus, the effects of changing the conditioning variable would be of some interest.

3. The standard $k-\varepsilon$ turbulence model is used in this study. Hence, it is worth to explore the effects of other turbulence modelling, such as RNG $k-\varepsilon$ [128], $k-\omega$ SST, Reynolds stress equation, etc., on the CMC prediction.

4. The effects of radiative heat loss on the CMC prediction.

5. Alternative models for the unconditional and conditional mean scalar dissipation rate for the conditioning variable.

6. Effects of alternative shapes for the PDF of the conditioning variable.

7. Effects of flame geometries on the CMC prediction. 


\section{Conclusions}

8. This study examined the premixed CMC for laboratory scale flames. Since, this gave encouraging results, it is worth to explore this methodology for practical combustion systems such as stationary gas turbine combustors.

9. Finally, implementing the premixed CMC in LES framework could prove to be worthwhile, since LES-CMC has been used in many previous studies for non-premixed flames.

Availability of mean species, temperature and velocity measurements with clearly specified errors and uncertainties from fully characterised premixed burners would be instrumental in validating and further developing models for turbulent lean premixed flames, and to support or unsupport the observation on the applicability of the $\mathrm{CMC}$ across the combustion regimes. 


\section{Appendix A}

\section{CMC equation for premixed combustion}

In this section the conditional moment closure equations are derived using the decomposition method [40]. The instantaneous mass fraction of species $\alpha$ is decomposed into conditional mean, $Q_{\alpha}$, and a fluctuation, $y_{\alpha}^{\prime \prime}$, as

$$
Y_{\alpha}\left(x_{i}, t\right)=Q_{\alpha}\left(\zeta ; x_{i}, t\right)+y_{\alpha}^{\prime \prime}\left(x_{i}, t\right)
$$

where the conditional average is defined as, $Q_{\alpha}\left(\zeta ; x_{i}, t\right)=\left\langle Y_{\alpha} \mid c=\zeta\right\rangle$, the ensemble average subject to the condition $c=\zeta$. The instantaneous progress variable is denoted as $c$, which is governed by

$$
\rho \frac{\partial c}{\partial t}+\rho u_{i} \frac{\partial c}{\partial x_{i}}=\frac{\partial}{\partial x_{i}}\left(\rho D_{c} \frac{\partial c}{\partial x_{i}}\right)+\dot{\omega}_{c}
$$

in the usual notation. The transport equation for the instantaneous scalar value, $Y_{\alpha}$, in the usual notations is

$$
\rho \frac{\partial Y_{\alpha}}{\partial t}+\rho u_{i} \frac{\partial Y_{\alpha}}{\partial x_{i}}=\frac{\partial}{\partial x_{i}}\left(\rho D_{\alpha} \frac{\partial Y_{\alpha}}{\partial x_{i}}\right)+\dot{\omega}_{\alpha}
$$


Substituting Eq. (1) into Eq. (3) gives

$$
\begin{gathered}
\frac{\partial Y_{\alpha}\left(x_{i}, t\right)}{\partial t}=\frac{\partial Q_{\alpha}}{\partial t}+\frac{\partial Q_{\alpha}}{\partial \zeta} \frac{\partial c}{\partial t}+\frac{\partial y_{\alpha}^{\prime \prime}}{\partial t}, \\
\frac{\partial Y_{\alpha}\left(x_{i}, t\right)}{\partial x_{i}}=\frac{\partial Q_{\alpha}}{\partial x_{i}}+\frac{\partial Q_{\alpha}}{\partial \zeta} \frac{\partial c}{\partial x_{i}}+\frac{\partial y_{\alpha}^{\prime \prime}}{\partial x_{i}} \\
\frac{\partial}{\partial x_{i}}\left(\rho D_{\alpha} \frac{\partial Y_{\alpha}}{\partial x_{i}}\right)=\frac{\partial}{\partial x_{i}}\left[\rho D_{\alpha}\left(\frac{\partial Q_{\alpha}}{\partial x_{i}}+\frac{\partial Q_{\alpha}}{\partial \zeta} \frac{\partial c}{\partial x_{i}}+\frac{\partial y_{\alpha}^{\prime \prime}}{\partial x_{i}}\right)\right] \\
=\overbrace{\frac{\partial}{\partial x_{i}}\left(\rho D_{\alpha} \frac{\partial Q_{\alpha}}{\partial x_{i}}\right)}+\overbrace{\frac{\partial}{\partial x_{i}}\left(\rho D_{\alpha} \frac{\partial Q_{\alpha}}{\partial \zeta} \frac{\partial c}{\partial x_{i}}\right)}^{3}+\overbrace{\frac{\partial}{\partial x_{i}}\left(\rho D_{\alpha} \frac{\partial y_{\alpha}^{\prime \prime}}{\partial x_{i}}\right)}
\end{gathered}
$$

The second term can written as

$$
\overbrace{\frac{\partial}{\partial x_{i}}\left(\rho D_{\alpha} \frac{\partial Q_{\alpha}}{\partial \zeta} \frac{\partial c}{\partial x_{i}}\right)}^{2}=\overbrace{\frac{\partial Q_{\alpha}}{\partial \zeta} \frac{\partial}{\partial x_{i}}\left(\rho D_{\alpha} \frac{\partial c}{\partial x_{i}}\right)}^{4}+\overbrace{\left(\rho D_{\alpha} \frac{\partial c}{\partial x_{i}}\right) \frac{\partial}{\partial x_{i}} \frac{\partial Q_{\alpha}}{\partial \zeta}}^{5}
$$

The fifth term can written as

$$
\overbrace{\left(\rho D_{\alpha} \frac{\partial c}{\partial x_{i}}\right) \frac{\partial}{\partial x_{i}} \frac{\partial Q_{\alpha}}{\partial \zeta}}^{5}=\overbrace{\rho D_{\alpha}\left(\frac{\partial c}{\partial x_{i}} \frac{\partial c}{\partial x_{i}}\right) \frac{\partial^{2} Q_{\alpha}}{\partial \zeta^{2}}}^{6}+\overbrace{\rho D_{\alpha} \frac{\partial c}{\partial x_{i}} \frac{\partial}{\partial x_{i}}\left(\frac{\partial Q_{\alpha}}{\partial \zeta}\right)}^{7}
$$

Now the diffusion term in Eq. (3) is the sum of the terms 1, 3, 4, 6 and 7

$$
\frac{\partial}{\partial x_{i}}\left(\rho D_{\alpha} \frac{\partial Y_{\alpha}}{\partial x_{i}}\right)=\overbrace{\frac{\partial}{\partial x_{i}}\left(\rho D_{\alpha} \frac{\partial Q_{\alpha}}{\partial x_{i}}\right)}^{1}+\overbrace{\frac{\partial Q_{\alpha}}{\partial \zeta} \frac{\partial}{\partial x_{i}}\left(\rho D_{\alpha} \frac{\partial c}{\partial x_{i}}\right)}^{4}+
$$




$$
\overbrace{\rho D_{\alpha}\left(\frac{\partial c}{\partial x_{i}} \frac{\partial c}{\partial x_{i}}\right) \frac{\partial^{2} Q_{\alpha}}{\partial \zeta^{2}}}^{6}+\overbrace{\rho D_{\alpha} \frac{\partial c}{\partial x_{i}} \frac{\partial}{\partial x_{i}}\left(\frac{\partial Q_{\alpha}}{\partial \zeta}\right)}^{7}+\overbrace{\frac{\partial}{\partial x_{i}}\left(\rho D_{\alpha} \frac{\partial y_{\alpha}^{\prime \prime}}{\partial x_{i}}\right)}^{3}
$$

Substituting Eqs. (4), (5) and (10) into Eq. (3) yields

$$
\begin{aligned}
\rho \frac{\partial Q_{\alpha}}{\partial t}+\rho u_{i} \frac{\partial Q_{\alpha}}{\partial x_{i}}- & \frac{\partial}{\partial x_{i}}\left(\rho D_{\alpha} \frac{\partial Q_{\alpha}}{\partial x_{i}}\right)-\rho D_{\alpha}\left(\frac{\partial c}{\partial x_{i}} \frac{\partial c}{\partial x_{i}}\right) \frac{\partial^{2} Q_{\alpha}}{\partial \zeta^{2}}- \\
\rho D_{\alpha} \frac{\partial c}{\partial x_{i}} \frac{\partial}{\partial x_{i}}\left(\frac{\partial Q_{\alpha}}{\partial \zeta}\right)+ & \frac{\partial Q_{\alpha}}{\partial \zeta}\left[\rho \frac{\partial c}{\partial t}+\rho u_{i} \frac{\partial c}{\partial x_{i}}-\frac{\partial}{\partial x_{i}}\left(\rho D_{\alpha} \frac{\partial c}{\partial x_{i}}\right)\right]+ \\
& \rho \frac{\partial y_{\alpha}^{\prime \prime}}{\partial t}+\rho u_{i} \frac{\partial y_{\alpha}^{\prime \prime}}{\partial x_{i}}-\frac{\partial}{\partial x_{i}}\left(\rho D_{\alpha} \frac{\partial y_{\alpha}^{\prime \prime}}{\partial x_{i}}\right)-\dot{\omega}_{\alpha}=0 .
\end{aligned}
$$

After some arrangement, the progress variable equation, Eq. (2), is rewritten as

$$
\rho \frac{\partial c}{\partial t}+\rho u_{i} \frac{\partial c}{\partial x_{i}}-\frac{\partial}{\partial x_{i}}\left(\rho D_{\alpha} \frac{\partial c}{\partial x_{i}}\right)=\frac{\partial}{\partial x_{i}}\left[\rho\left(D_{c}-D_{\alpha}\right) \frac{\partial c}{\partial x_{i}}\right]+\dot{\omega}_{c} .
$$

Substituting Eq. (12) into Eq. (11) yields

$$
\begin{array}{r}
\rho \frac{\partial Q_{\alpha}}{\partial t}+\rho u_{i} \frac{\partial Q_{\alpha}}{\partial x_{i}}-\frac{\partial}{\partial x_{i}}\left(\rho D_{\alpha} \frac{\partial Q_{\alpha}}{\partial x_{i}}\right)-\rho D_{\alpha}\left(\frac{\partial c}{\partial x_{i}} \frac{\partial c}{\partial x_{i}}\right) \frac{\partial^{2} Q_{\alpha}}{\partial \zeta^{2}}- \\
\rho D_{\alpha} \frac{\partial c}{\partial x_{i}} \frac{\partial}{\partial x_{i}}\left(\frac{\partial Q_{\alpha}}{\partial \zeta}\right)+\frac{\partial Q_{\alpha}}{\partial \zeta}\left[\frac{\partial}{\partial x_{i}}\left[\rho\left(D_{c}-D_{\alpha}\right) \frac{\partial c}{\partial x_{i}}\right]+\dot{\omega}_{c}\right]+ \\
\rho \frac{\partial y_{\alpha}^{\prime \prime}}{\partial t}+\rho u_{i} \frac{\partial y_{\alpha}^{\prime \prime}}{\partial x_{i}}-\frac{\partial}{\partial x_{i}}\left(\rho D_{\alpha} \frac{\partial y_{\alpha}^{\prime \prime}}{\partial x_{i}}\right)-\dot{\omega}_{\alpha}=0
\end{array}
$$

Taking conditional average of Eq. (13) yields,

$$
\begin{gathered}
\langle\rho \mid \zeta\rangle \frac{\partial Q_{\alpha}}{\partial t}+\left\langle\rho u_{i} \mid \zeta\right\rangle \frac{\partial Q_{\alpha}}{\partial x_{i}}-\left\langle\rho D_{\alpha} \frac{\partial c}{\partial x_{i}} \frac{\partial c}{\partial x_{i}} \mid \zeta\right\rangle \frac{\partial^{2} Q_{\alpha}}{\partial \zeta^{2}}= \\
\left\langle\left[\frac{\partial}{\partial x_{i}}\left(\rho D_{\alpha} \frac{\partial Q_{\alpha}}{\partial x_{i}}\right)+\rho D_{\alpha} \frac{\partial c}{\partial x_{i}} \frac{\partial}{\partial x_{i}}\left(\frac{\partial Q_{\alpha}}{\partial \zeta}\right)-\frac{\partial Q_{\alpha}}{\partial \zeta} \frac{\partial}{\partial x_{i}}\left(\rho\left(D_{c}-D_{\alpha}\right) \frac{\partial c}{\partial x_{i}}\right)\right] \mid \zeta\right\rangle
\end{gathered}
$$




$$
-\left\langle\left[\rho \frac{\partial y_{\alpha}^{\prime \prime}}{\partial t}+\rho u_{i} \frac{\partial y_{\alpha}^{\prime \prime}}{\partial x_{i}}-\frac{\partial}{\partial x_{i}}\left(\rho D_{\alpha} \frac{\partial y_{\alpha}^{\prime \prime}}{\partial x_{i}}\right)\right] \mid \zeta\right\rangle+\left\langle\dot{\omega}_{\alpha} \mid \zeta\right\rangle-\frac{\partial Q_{\alpha}}{\partial \zeta}\left\langle\dot{\omega}_{c} \mid \zeta\right\rangle
$$

It is to be taken that density weighted conditional averaging [40] is used and the rules of differentiating the conditional averages as ex-positional in [129] are employed. Rearranging the above equation gives

$$
\begin{array}{r}
\langle\rho \mid \zeta\rangle \frac{\partial Q_{\alpha}}{\partial t}+\left\langle\rho u_{i} \mid \zeta\right\rangle \frac{\partial Q_{\alpha}}{\partial x_{i}}-\frac{\mathrm{Le}_{c}}{\mathrm{Le}_{\alpha}}\left\langle\rho N_{c} \mid \zeta\right\rangle \frac{\partial^{2} Q_{\alpha}}{\partial \zeta^{2}}=\left\langle\dot{\omega}_{\alpha} \mid \zeta\right\rangle-\left\langle\dot{\omega}_{c} \mid \zeta\right\rangle \frac{\partial Q_{\alpha}}{\partial \zeta}+ \\
e y_{\alpha}+e_{Q_{\alpha}}
\end{array}
$$

where

$$
e_{y_{\alpha}} \equiv-\left\langle\left[\rho \frac{\partial y_{\alpha}^{\prime \prime}}{\partial t}+\rho u_{i} \frac{\partial y_{\alpha}^{\prime \prime}}{\partial x_{i}}-\frac{\partial}{\partial x_{i}}\left(\rho D_{\alpha} \frac{\partial y_{\alpha}^{\prime \prime}}{\partial x_{i}}\right)\right] \mid \zeta\right\rangle
$$

and

$$
\begin{array}{r}
e_{Q_{\alpha}} \equiv\left\langle\frac{\partial}{\partial x_{i}}\left(\rho D_{\alpha} \frac{\partial Q_{\alpha}}{\partial x_{i}}\right)+\rho D_{\alpha} \frac{\partial c}{\partial x_{i}} \frac{\partial}{\partial x_{i}}\left(\frac{\partial Q_{\alpha}}{\partial \zeta}\right) \mid \zeta\right\rangle+ \\
\left\langle\frac{\partial Q_{\alpha}}{\partial \zeta} \frac{\partial}{\partial x_{i}}\left[\left(1-\frac{L e_{\alpha}}{L_{\mathrm{c}}}\right) \rho D_{\alpha} \frac{\partial c}{\partial x_{i}}\right] \mid \zeta\right\rangle
\end{array}
$$

The conditional dissipation rate is given by $N_{c}=\left\langle D_{c}(\nabla c \cdot \nabla c) \mid \zeta\right\rangle$ and $\left\langle\rho N_{c} \mid \zeta\right\rangle=$ $\langle\rho \mid \zeta\rangle\left\langle N_{c} \mid \zeta\right\rangle[130]$. The Lewis number of species $\alpha$ is denoted by $L e_{\alpha}$. 


\section{Bibliography}

[1] A. R. Masri., R. W. Dibble., and R. S. Barlow. The structure of turbulent non-premixed flames of methanol over a range of mixing rates. Combust. Flame, 89(2):167-185, 1992. x, 42

[2] Y. C. Chen, N. Peters, G. A. Schneemann, N. Wruck, U. Renz, and M. S. Mansour. The detailed flame structure of highly stretched turbulent premixed Methane-air flames. Combust. Flame, 3:223-226, 1996. xi, 6, 70, 71, $72,74,75,136,137$

[3] M. J. Dunn, A. R. Masri, and R. W. Bilger. A new piloted premixed jet burner to study strong finite-rate chemistry effects. Combust. Flame, 151(1-2):46 - 60, 2007. xiii, 6, 102, 103, 106, 136, 138

[4] M. J. Dunn. Finite-rate chemistry effects in turbulent premixed combustion. PhD thesis, The University of Sydney, Australia, 2008. xiii, xiv, xv, 103, $108,122,123,124,125,126,127,128,129,130,131,132,133,134,138$

[5] D. H. Rowinski and S. B. Pope. PDF calculations of piloted premixed jet flames. Combust. Theory Modelling, 15(2):245-266, 2011. xiv, xv, 104, 118, $123,124,129,130,131,132,138$ 
[6] M. J. Dunn, A. R. Masri, R. W. Bilger, and R. S. Barlow. Finite rate chemistry effects in highly sheared turbulent premixed flames. Flow Turb. Combust., 85:621-648, 2010. xiv, xv, 104, 108, 122, 123, 124, 125, 126, 127, $128,129,130,131,132,133,134$

[7] R. A. Houghton. The contemporary Carbon cycle. In D. H. Holland and K. K. Turekian, editors, Treatise on Geochemistry, pages 473 - 513. Pergamon, Oxford, 2003. 2

[8] M. Maslin. Global warming: Causes, effects and the future. LLC and Voyageur Press, Minneapolis, USA, 2007. 2

[9] C. N. Lane. Acid rain: Overview and Abstracts. Nova Science Publisher, inc, New York, USA, 2003. 2

[10] European Commission Statistics. Renewable energy statistics, September 2010. 2

[11] S. M. Correa. A review of NOx formation under gasturbine combustion conditions. Combust. Sci. Tech., 87:329362, 1992. 3

[12] J. B. Heywood. Pollutant formation and control in sparkignition engines. Prog. Energy Combust. Sci., 1:135164, 1976. 3

[13] J. F. Driscoll. Turbulent premixed combustion: Flamelet structure and its effect on turbulent burning velocities. Prog. Energy Combust. Sci., 34(1):91 $-134,2008.3,28$

[14] B. S. Brewster, S. M. Cannon, J. R. Farmer, and F. Meng. Modeling of lean 
premixed combustion in stationary gas turbines. Prog. Energy Combust. Sci., 25(4):353-385, 1999. 3

[15] Y. Huang and V. Yang. Dynamics and stability of lean-premixed swirlstabilized combustion. Prog. Energy Combust. Sci., 35(4):293 - 364, 2009. 3

[16] N. Swaminathan and K. N. C. Bray. Turbulent premixed flames. Cambridge University Press, Cambridge, UK, 2011. 3, 8, 16, 17, 21, 23, 25, 27, 31, 34, 36,41

[17] D. Veynante and L. Vervisch. Turbulent combustion modelling. Prog. Energy Combust. Sci., 28(3):193 - 266, 2002. 3, 5, 14, 26, 34, 43, 49

[18] D. Dunn Rankin. Lean combustion technology and control. Elsevier, California, USA, 1st. edition, 2008. 3

[19] T. Lieuwen, H. Torres, C. Johnson, and B. T. Zinn. A mechanism of combustion instability in lean premixed gas turbine combustors. J. Eng. Gas Turb. Power, 123(1):182-189, 2001. 3

[20] T. Echekki and E. Mastorakos. Turbulent Combustion Modeling: Advances, new trends and perspectives. Springer, New York, USA, 2010. 4, 26, 34

[21] H. Tennekes and J. L. Lumley. A first course in turbulence. The Massachusetts Inst. of Technology, Boston, USA, 1972. 4

[22] T. Poinsot and D. Veyante. Theoretical and numerical combustion. Westview Press, Philadelphia, USA, 2nd. edition, 2005. 4, 8, 10, 13, 14, 17, 18, $21,22,23,25,26,34$ 
[23] S. B. Pope. Turbulent flows. Cambridge University Press, Cambridge, UK, 2000. $4,8,10,22$

[24] F. A. Williams. Turbulent combustion. In J. D. Buckmaster, editor, The mathematics of combustion, pages 97-132. Society for Industrial and Applied Mathematics (SIAM), Philadelphia, USA, 1985. 5, 26, 37, 49

[25] R. W. Bilger. Future progress in turbulent combustion research. Prog. Energy Combust. Sci., 26:367-380, 2000. 5, 8

[26] R. W. Bilger, S. B. Pope, K. N. C. Bray, and J. F. Driscoll. Paradigms in turbulent combustion research. Proc. Combust. Inst., 30:21-42, 2005. 5, 8, 9

[27] R. W. Bilger. Conditional Moment Closure for turbulent reacting flow. Phys. Fluids A, 5(2):436-444, 1993. 6, 38, 39, 41, 43

[28] A. Yu. Klimenko. Multi-component diffusion of various admixtures in turbulent flow. Fluid Dynamics., 25:327-334, 1990. 6, 41, 43

[29] S. B. Pope. PDF methods for turbulent reactive flows. Prog. Energy Combust. Sci., 11(2):119 - 192, 1985. 6, 35, 49

[30] M. J. Cleary, J. H. Kent, and R. W. Bilger. Prediction of Carbon Monoxide in fires by Conditional Moment Closure. Proc. Combust. Inst., 29:273-279, 2002.Adelaide, Australia,. 6, 41, 135

[31] I. S. Kim and E. Mastorakos. Simulations of turbulent lifted jet flames with two-dimensional conditional Moment Closure. Proc. Combust. Inst., 30(1):911 - 918, 2005. 6, 41, 135 
[32] J. W. Rogersson. Measurements and modelling of a Bagasse-fired boiler. PhD thesis, The University of Sydney, Sydney, Australia, 2006. 6, 41, 45, $61,69,135$

[33] S. H. Kim, K. Y. Huh, and L. Tao. Application of the elliptic Conditional Moment Closure model to a two-dimensional non-premixed methanol bluffbody flame. Combust. Flame, 120:75-90, 1999. 6, 41, 135

[34] S. Sreedhara and Kang. Y. Huh. Modelling of turbulent two-dimensional non premixed Methane-Hydrogen flame over a bluff-body using first and second order elliptic Conditional Moment Closure. Combust. Flame, 143:119-134, 2005. 6, 41, 135

[35] Y. M. Wright, G. De Paola, K. Boulouchos, and E. Mastorakos. Simulations of spray autoignition and flame establishment with two-dimensional CMC. Combust. Flame, 143(4):402 - 419, 2005. 6, 41, 135

[36] Y. Yunardi, R. M. Woolley, and M. Fairweather. Conditional Moment Closure prediction of soot formation in turbulent non-premixed ethylene flames. Combust. Flame, 152:360-376, 2008. 6, 41, 135

[37] F. A. Williams. Combustion theory. Perseus Books, USA, 2nd. edition, 1985. 8,10

[38] J. Warnatz, U. Maas, and R. W. Dibble. Combustion physical and chemical fundamentals, modelling and simulation, experiments, pollutant formation. Springer, New York, USA, 4th. edition, 2006. 8, 10 
[39] E. Mastorakos and R. S. Cant. An introduction to turbulent reacting flows. Imperial College Press, London, UK, 2008. 8

[40] R. W. Bilger and A.Y. Klimenko. Conditional Moment Closure for turbulent combustion. Prog. Energy Combust. Sci., 25:595-687, 1999. 14, 38, 40, $41,43,45,46,47,49,71,141,144$

[41] A. Neophytou, E. Mastorakos, and R.S. Cant. DNS of spark ignition and edge flame propagation in turbulent droplet-laden mixing layers. Combust. Flame, 157(6):1071 - 1086, 2010. 17

[42] P. Schroll, A. P. Wandel, R. S. Cant, and E. Mastorakos. Direct Numerical Simulations of autoignition in turbulent two-phase flows. Proc. Combust. Inst., 32(2):2275 - 2282, 2009. 17

[43] M. Klein, N. Chakraborty, and R. Cant. Effects of turbulence on selfsustained combustion in premixed flame kernels: A Direct Numerical Simulation DNS study. Flow Turb. Combust., 81:583-607, 2008. 17

[44] J. G. and Wissink. DNS of separating, low Reynolds number flow in a turbine cascade with incoming wakes. Int. J. Heat and Fluid Flow, 24(4):626 $-635,2003.17$

[45] A. Triantafyllidis, E. Mastorakos, and R. L. G. M. Eggels. Large Eddy Simulations of forced ignition of a non-premixed bluff-body Methane flame with Conditional Moment Closure. Combust. Flame, 156(12):2328 - 2345, 2009. 18

[46] M. W. A. Pettit, B. Coriton, A. Gomez, and A. M. Kempf. Large Eddy 
Simulation and experiments on non-premixed highly turbulent opposed jet flows. Proc. Combust. Inst., 33(1):1391 - 1399, 2011. 18

[47] V. D. Sarli, A. D. Benedetto, and G. Russo. Sub-grid scale combustion models for Large Eddy Simulation of unsteady premixed flame propagation around obstacles. J. Hazardous Materials, 180(1-3):71 - 78, 2010. 18

[48] Y. Y. Wu, C. K. Chan, and L. X. Zhou. Large Eddy Simulation of an ethyleneair turbulent premixed v-flame. J. Computational Applied Math., 235(13):3768 - 3774, 2011. 18

[49] P. Wang and X. S. Bai. Large Eddy Simulation of turbulent premixed flames using level-set G-equation. Proc. Combust. Inst., 30(1):583 - 591, 2005. 18

[50] W. C. Reynolds. Computation of turbulent flows. Annual Review Fluid Mech., 8(1):183-208, 1976. 22

[51] W. P. Jones and B. E. Launder. The prediction of laminarization with a twoequation model of turbulence. Int. J. Heat and Mass Transfer, 15(2):301 314, 1972. 22, 23

[52] W. P. Jones. Turbulence modelling and numerical solution methods for variable density and combusting flows. In P. A. Libby and F. A. Williams, editors, Turbulent reacting flows, pages 309 - 368. Academic Press, London, 1994. 23,26

[53] O. R. Darbyshire. Modelling of turbulent stratified flames. PhD thesis, The University of Cambridge, Cambridge, UK, 2011. 23 
[54] B. E. Launder, G. J. Reece, and W. Rodi. In the development of a Reynolds stress turbulence closure. J. Fluid Mech., 41:537-566, 1975. 23

[55] C. G. Spezilae and T. B. Gatski. Modelling the pressure-strain correlation of turbulence:an invariant dynamical systems approach. J. Fluid Mech., $227: 245-272,1991.23$

[56] D. B. Taulbee. An improved algebraic Reynolds stress model and corresponding non-linear stress model. Phys. Fluids A, 4:2555-2560, 1992. 23

[57] N. Swaminathan, R.W. Bilger, and B. Cuenot. Relationship between turbulent scalar flux and conditional dilatation in premixed flames with complex chemistry. Combust. Flame, 126(4):1764 - 1779, 2001. 24

[58] K. N. C. Bray. Turbulent transport in flames. Proc. Royal Soc. London. Series A: Math. Phys. Sci., 451(1941):231-256, 1995. 24, 31

[59] K.N.C. Bray, Paul A. Libby, and J.B. Moss. Unified modeling approach for premixed turbulent combustionpart I: General formulation. Combust. Flame, 61(1):87 - 102, 1985. 24

[60] K. N. C. Bray, M. Champion, and P. A. Libby. Premixed flames in stagnating turbulence part IV: A new theory for the Reynolds stresses and Reynolds fluxes applied to impinging flows. Combust. Flame, 120(1-2):1 18, 2000. 24

[61] K. N. C. Bray, P. A. Libby, G. Masuya, and J. B. Moss. Turbulence production in premixed turbulent flames. Combust. Sci. Tech., 25(3-4):127-140, 1981. 24 
[62] J. B. Moss. Simultaneous measurements of concentration and velocity in an open premixed turbulent flame. Combust. Sci. Tech., 22(3-4):119-129, 1980. 24

[63] R. K. Cheng and I. G. Shepherd. The influence of burner geometry on premixed turbulent flame propagation. Combust. Flame, 85(1-2):7 - 26, 1991. 24

[64] I. G. Shepherd, J. B. Moss, and K. N. C. Bray. Turbulent transport in a confined premixed flame. 19th Symp Int Combust., 19(1):423 - 431, 1982. 24

[65] J. H. Frank, P. A.M. Kalt, and R. W. Bilger. Measurements of conditional velocities in turbulent premixed flames by simultaneous OH PLIF and PIV. Combust. Flame, 116(1-2):220 - 232, 1999. 24

[66] K. N. C. Bray D. Veynante, A. Trouve and T. Mantel. Gradient and counter-gradient scalar transport in turbulent premixed flames. J. Fluid Mech., 332:263-293, 1997. 24, 25

[67] N. Swaminathan, R. W. Bilger, and G. R. Ruetsch. Interdependence of the instantaneous flame front structure and the overall scalar flux in turbulent premixed flames. Combust. Sci. Tech., 128(1-6):73-97, 1997. 24

[68] R. P. Lindstedt and E. M. Vaos. Transported PDF modeling of highReynolds number premixed turbulent flames. Combust. Flame, 145:495511, 2006. 25, 70, 89, 137

[69] N. Swaminathan and K. N. C. Bray. In N. Swaminathan and K. N. C. 
Bray, editors, Turbulent premixed flames, pages 1-33. Cambridge University Press, Cambridge, UK, 2011. 26

[70] N. Peters. Turbulent combustion. Cambridge University Press, Cambridge, UK. $27,28,30,37$

[71] R. S. Cant and E. Mastorakos. Introduction to turbulent reacting flows. Imperial College Press, London, UK, 2007. 27, 30

[72] D. B. Spalding. Mixing and chemical reaction in steady confined turbulent flames. Proc. Combust. Inst, 13:649-657, 1970. 29

[73] K. N. C. Bray and P. A. Libby. Interaction effects in turbulent premixed flames. Phys. Fluids, 19:1687-1701, 1976. 30

[74] K. N. C. Bray and J. B. Moss. A unified statistical model of the premixed turbulent flame. Acta Astronautica, 4:291-319, 1977. 30, 31

[75] K. N. C. Bray. Turbulent flows with premixed reactants. In P. A. Libby and F. A. Williams, editors, Turbulent Reacting Flows, page 115183. SpringerVerlag, New York, 1980. 30, 31

[76] K. N. C. Bray. The interaction between turbulence and combustion. 17th Symp. Int. Combust. The Combustion Institute, Pittsburgh, 17(1):223-223, 1979. 31

[77] K. N. C. Bray, P. A. Libby, and J. B. Moss. Flamelet crossing frequencies and mean reaction rates in premixed turbulent combustion. Combust. Sci. Tech., 41:143-172, 1984. 32 
[78] D. Bradley. How fast can we burn? 24th Symp. Int. Combust. The Combustion Institute, University of Sydney, Australia, 24(1):247 - 262, 1992. 32

[79] H. Kolla and N. Swaminathan. Strained flamelets for turbulent premixed flames I: Formulation and planar flame results. Combust. Flame, 157:943954, 2010. 33, 41, 49, 51, 52, 70, 73, 89, 136, 137, 138

[80] H. Kolla and N. Swaminathan. Strained flamelets for turbulent premixed flames II: Laboratory flame results. Combust. Flame, 157:12741289, 2010. 33,45

[81] F. E. Marble and J. E Broadwell. The coherent flame model for turbulent chemical reactions. Project SQUID, technical report TRW-9-PU, 1977. Purdue University. 33

[82] S. M. Candel and T. J. Poinsot. Flame stretch and the balance equation for the flame area. Combust. Sci. Tech., 70:1-15, 1990. 33, 34

[83] S. B. Pope. The evolution of surfaces in turbulence. Int. J. Eng. Sci., 26(5):445 - 469, 1988. 33, 34

[84] K. N. C. Bray and P. A. Libby. Recent development in the BML model of premixed turbulent combustion. In P. A. Libby and F. A. Williams, editors, Turbulent reacting flows, pages 115 - 147. Academic Press, London, 1994. 33,34

[85] A. Trouve and T. Poinsot. The evolution equation for the flame surface density. J. Fluid Mech., 278:1 - 31, 1994. 33, 34 
[86] E. V. Kalmthout and D. Veynante. Direct Numerical Simulations analysis of flame surface density models for non premixed turbulent combustion. Phys. Fluids, 10(9):2347, 1998. 33

[87] E. E. OBrien. The probability density function PDF approach to reacting turbulent flows. Turbulent Reacting Flows, pages 185-218, 1980. 35

[88] D. C. Haworth. Progress in probability density function methods for turbulent reacting flows. Prog. Energy Combust. Sci., 36(2):168 - 259, 2010. 36

[89] D. C. Haworth and S. B. Pope. Transported Probability Density Function Methods for Reynolds-Averaged and Large-Eddy Simulations. In Tarek Echekki and Epaminondas Mastorakos, editors, Turbulent Combustion Modeling, volume 95, pages 119-142. Springer, New York, USA, 2011. 36

[90] R. P. Lindstedt. Transported probability density function methods for premixed turbulent flames. In N. Swaminathan and K. N. C. Bray, editors, Turbulent premixed flames, pages 102-133. Cambridge University Press, Cambridge, UK, 2011. 36

[91] S. S. Girimaji. Assumed $\beta$-PDF model for turbulent mixing: Validation and extension to multiple scalar mixing. Combust. Sci. Tech., 78(4-6):177-196, 1991. 36

[92] K. N. C. Bray and N. Peters. Laminar flamelets in turbulent flames. In P. A Libby and F. A. Williams, editors, Turbulent Reacting Flows, pages 63-113. Academic, London, UK, 1994. 37 
[93] F. A. Williams. Some recent studies in turbulent combustion. In A. Yoshida, editor, Smart control of turbulent combustion, pages 1-12. Springer-Verlag, Tokyo, Japan, 2001. 37

[94] S. M. Martin. An improved Conditional Moment Closure model. presented at the Third Joint Meeting of the U.S. Sections of the Combus. Inst., Chicago, 2003. 41

[95] S. M. Martin, J. C. Kramlich, G. Kosaly, and J. J. Riley. The premixed Conditional Moment Closure method applied to idealized lean premixed gas turbine combustors. J. Eng. Gas Turb. Power, 125(4):895-900, 2003. 41

[96] N. Swaminathan and R. W. Bilger. Analysis of conditional Moment Closure for turbulent premixed flames. Combust. Theory Modelling, 5:241-260, 2001. $41,43,46,47$

[97] N. Swaminathan and K.N.C. Bray. Effect of dilatation on scalar dissipation in turbulent premixed flames. Combust. Flame, 143(4):549 - 565, 2005. 41, 49,50

[98] H. Kolla, J. W. Rogerson, N. Chakraborty, and N. Swaminathan. Scalar dissipation rate modelling and its validation. Combust. Sci. Tech., 181(3):518535, 2009. 41, 49, 50

[99] N. Chakraborty, J. W. Rogerson, and N. Swaminathan. A prior assessment of closures for scalar dissipation rate transport in turbulent premixed flames using Direct Numerical Simulation. Phys. Fluids, 20(4):045106, 2008. 41, 50 
[100] E. S. Richardson, N. Chakraborty, and E. Mastorakos. Analysis of Direct Numerical Simulations of ignition fronts in turbulent non-premixed flames in the context of Conditional Moment Closure. Proc. Combust. Inst., 31(1):1683 - 1690, 2007. 45

[101] A. Simon and E. Mastorakos. Conditional Moment Closure/Large Eddy Simulation of the Delft-III natural gas non-premixed jet flame. Flow Turb. Combust., 88:207-231, 2012. 45

[102] N. Peters. Laminar diffusion flamelet models in non-premixed turbulent combustion. Prog. Energy Combust. Sci., 10:319-339, 1984. 49

[103] S. Sreedhara, Y. Lee, Kang Y. Huh, and D.H. Ahn. Comparison of sub models for conditional velocity and scalar dissipation in CMC simulation of piloted jet and bluff-body flames. Combust. Flame, 152(1-2):282 - 286, 2008. 49

[104] P. A. Libby and F. A. Williams. Fundamental aspects and a review. In P. A. Libby and F. A. Williams, editors, Turbulent reacting flows, pages 1 - 62. Academic Press, London, 1994. 49

[105] Y. C. Chen and M. S. Mansour. Measurements of scalar dissipation in turbulent Hydrogen diffusion flames and some implications on combustion modeling. Combust. Sci. Tech., 126(1-6):291-313, 1997. 49

[106] S. H. Starner, R. W. Bilger, M. B. Long, J. H. Frank, and D. F. Marran. Scalar dissipation measurements in turbulent jet diffusion flames of air diluted Methane and Hydrogen. Combust. Sci. Tech., 129(1-6):141-163, 1997. 49 
[107] F. O'Young and R. W. Bilger. Measurement of scalar dissipation in premixed flames. Combust. Sci. Tech.., 113(1):393-411, 1996. 49

[108] N. Swaminathan and R. W. Bilger. Scalar dissipation, diffusion and dilation in turbulent H2-air premixed flames with complex chemistry. Combust. Theory Model., 5(1):429-446, 2001. 49

[109] T. Mantel and R. W. Bilger. Some conditional statistics in a turbulent premixed flame derived from Direct Numerical Simulations. Combust. Sci. Tech., 110(1):393-417, 1995. 49

[110] N. Chakraborty, M. Chapion, A. Mura, and N. Swaminathan. Scalar dissipation rate approach. In N. Swaminathan and K. N. C. Bray, editors, Turbulent premixed flames, pages 74-102. Cambridge University Press, Cambridge, UK, 2011. 50

[111] A. N. Tikhonov, A. Goncharsky, V. V. Stepanov, and A. G. Yagola. Numerical method for the solution of ill-posed problems. Kluwer Academic Publishers, Moscow, Russia, 1990. 52, 53

[112] T. Dunstan, N. Swaminathan, K. N. C. Bray, and S. R. Cant. Geometrical properties and turbulent flame speed measurements in stationary premixed v-flames using Direct Numerical Simulation. Flow Turb. Combust., 87:237259, 2011. 54

[113] J. W. Rogerson, N. Swaminathan, M. Tanahashi, and N. Shiwaku. Analysis of progress variable variance equations using DNS data. Proc. European Meeting, 2007. 55 
[114] Y. Nada, M. Tanahashi, and T. Miyauchi. Effect of turbulence characteristics on local flame structure of H2-air premixed flames. J. Turb., 5:16, 2004. 55

[115] A. N. Tikhonov, A. V. Goncharsky, and A. G. Yagola. Algorithms and programs for the solution of linear ill-posed problems. Moscow State UniversityFaculty of Physics. http://foroff.phys.msu.ru/Illposed/eng/, 1995. 58

[116] S. V. Patankar. Numerical heat transfer and fluid flow. Hemisphere Publishing Corporation, Washington, USA, 1980. 61, 63, 68

[117] J. W. Rogerson, J. H. Kent, and R. W. Bilger. Conditional Moment Closure in bagasse-fired boiler. Proc. Combust. Ins., 31:2805-2811, 2007. 66, 69

[118] A. Mura, F. Galzin, and R. Borghi. A unified PDF-flamelet model for turbulent premixed combustion. Combust. Sci. Tech., 175(9):1573-1609, 2003. 70,137

[119] R. O. P. Prasad and J. P. Gore. An evaluation of flame surface density models for turbulent premixed jet flames. Combust. Flame, 16:1-14, 1999. $70,89,137$

[120] M. Herrmann. Numerical simulation of turbulent bunsen flames with a level set flamelet model. Combust. Flame, 145:357-375, 2006. 70, 89, 137

[121] G. Wang, M. Boileau, and D. Veynante. Implementation of a dynamic thickened flame model for large Eddy Simulations of turbulent premixed combustion. Combust. Flame, 158(11):2199 - 2213, 2011. 70, 89, 137 
[122] S. B. Pope. An explanation of the turbulent round-jet/plane-jet anomaly. AIAA J., 16(3):279 - 281, 1978. 74

[123] G. P. Smith, D. M. Golden, M. Frenklach, N. W. Moriarty, B. Eiteneer, M. Goldenberg, C. T. Bowman, R. K. Hanson, S. Song, W. C. Gardiner, Jr., V. V. Lissianski, and Z. Qin. Gri-mech 3.0. The Gas Research Inst., 2011. GRI-Mech 3.0 available at: http://www.me.berkeley.edu/grimech. 75, 108

[124] M. D. Smooke. Lecture notice in physics. pringer-Verlag, 1991. 75

[125] M. J. Dunn, A. R. Masri, R. W. Bilger, R. S. Barlow, and G. H. Wang. The compositional structure of highly turbulent piloted premixed flames issuing into a hot co-flow. Proc. Combust. Inst., 32(2):1779-1786. 104

[126] C. Duwig, K. J. Nogenmyr, C. K. Chan, and M. J. Dunn. Large Eddy Simulations of a piloted lean premix jet flame using finite-rate chemistry. Combust. Theory Modelling, 15(4):537-568, 2011. 104

[127] J. W. Rogerson. Measurements and Modelling of a Bagasse-Fired Boiler. PhD thesis, The University of Sydney, Australia, 2006. 136

[128] V. Yakhot, S. A. Orszag, S. Thangam, T. B. Gatski, and C. G. Speziale. Development of turbulence models for shear flows by a double expansion technique. Phys. Fluids A: Fluid Dynamics, 4(7):1510-1520, 1992. 139

[129] A. Yu. Klimenko and R. W. Bilger. Conditional Moment Closure for turbulent combustion. Prog. Energy Combust. Sci., 25:595-687, 1999. 144

[130] N. Swaminathan and R. W. Bilger. Scalar dissipation, diffusion and dilata- 
tion in turbulent H2-air premixed flames with complex chemistry. Combust. Theory Model., 5(3):429-446, 2001. 144 